\title{
Directional approach to enantiomerically enriched functionalized [7]oxa- helicenoids and groove-based selective cyanide sensing
}

Vaibhav N. Khose, ${ }^{\mathrm{a}}$ Mohammed Hasan, ${ }^{\mathrm{a}, \mathrm{b}}$ Sushil C. Khot, ${ }^{\mathrm{a}}$ Shaikh M. Mobin, ${ }^{\mathrm{c}}$ Victor Borovkov*b and Anil V. Karnik*a

${ }^{a}$ Department of Chemistry, University of Mumbai, Vidayanagari, Santacruz (East), Mumbai 400098, India

${ }^{\mathrm{b}}$ College of Chemistry and Materials Science, South Central University for Nationalities, 182\# Minzu RD, Hongshan District, Wuhan, Hubei province, 430074, China

${ }^{c}$ Department of Chemistry, Indian Institute of Technology Indore, Simrol, Khandwa Road, Indore 453552, India

\section{SUPPORTING INFORMATION CONTENTS}

\begin{tabular}{|ccc|}
\hline Sr. No. & Title & Page Numbers \\
\hline 1. & Spectral data & S2-S36 \\
\hline 2. & X-ray crystallography data & S37-S38 \\
\hline 3. & HPLC chromatogram & S39-S47 \\
\hline 4. & Chiroptical studies & S48-S55 \\
\hline 5. & Fluorescence spectra & S55-S56 \\
\hline 6. & Sensing data & S57-S74 \\
\hline
\end{tabular}




\section{Spectral Data}

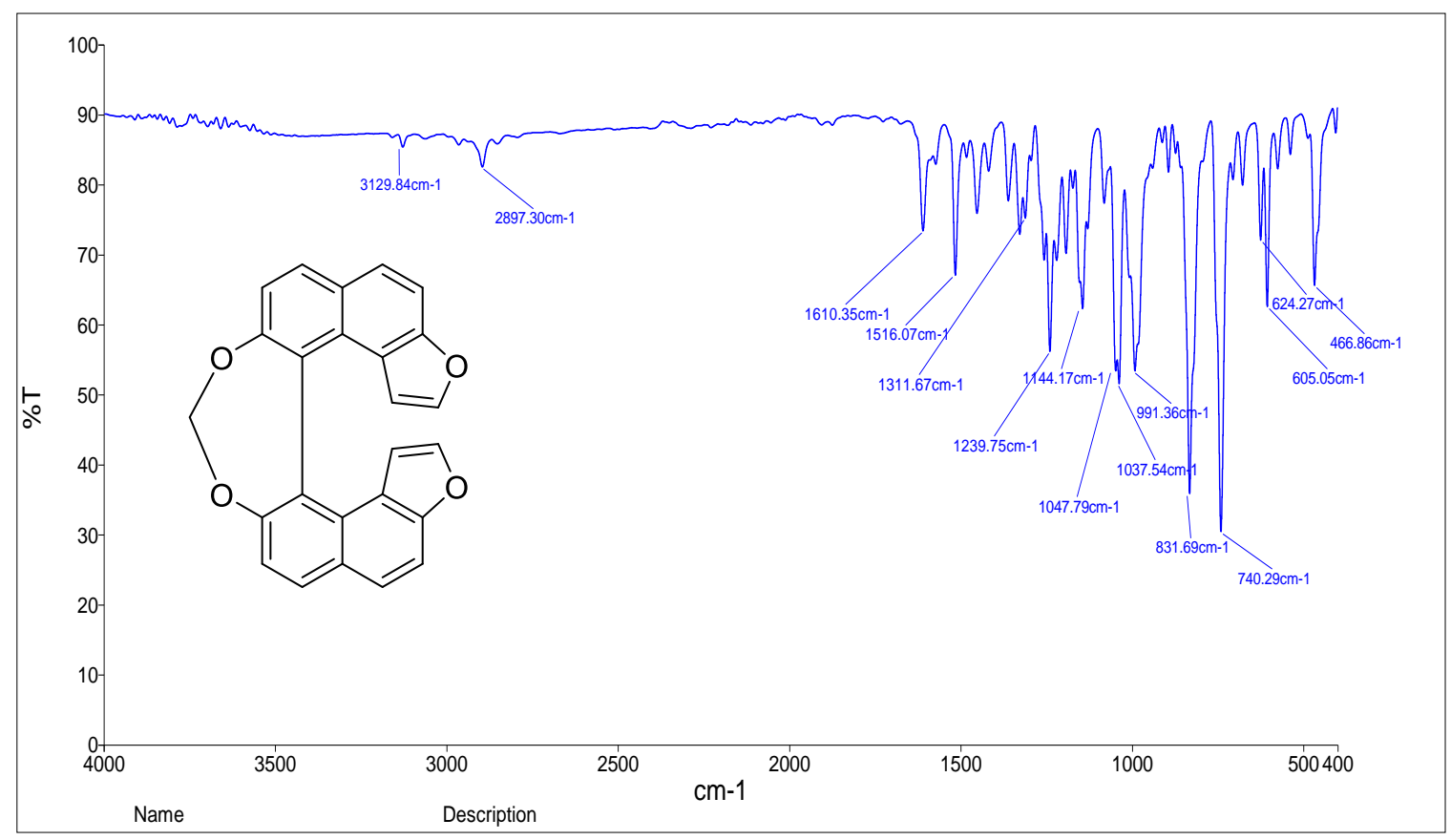

Figure S1. IR of helicenoid, 2.

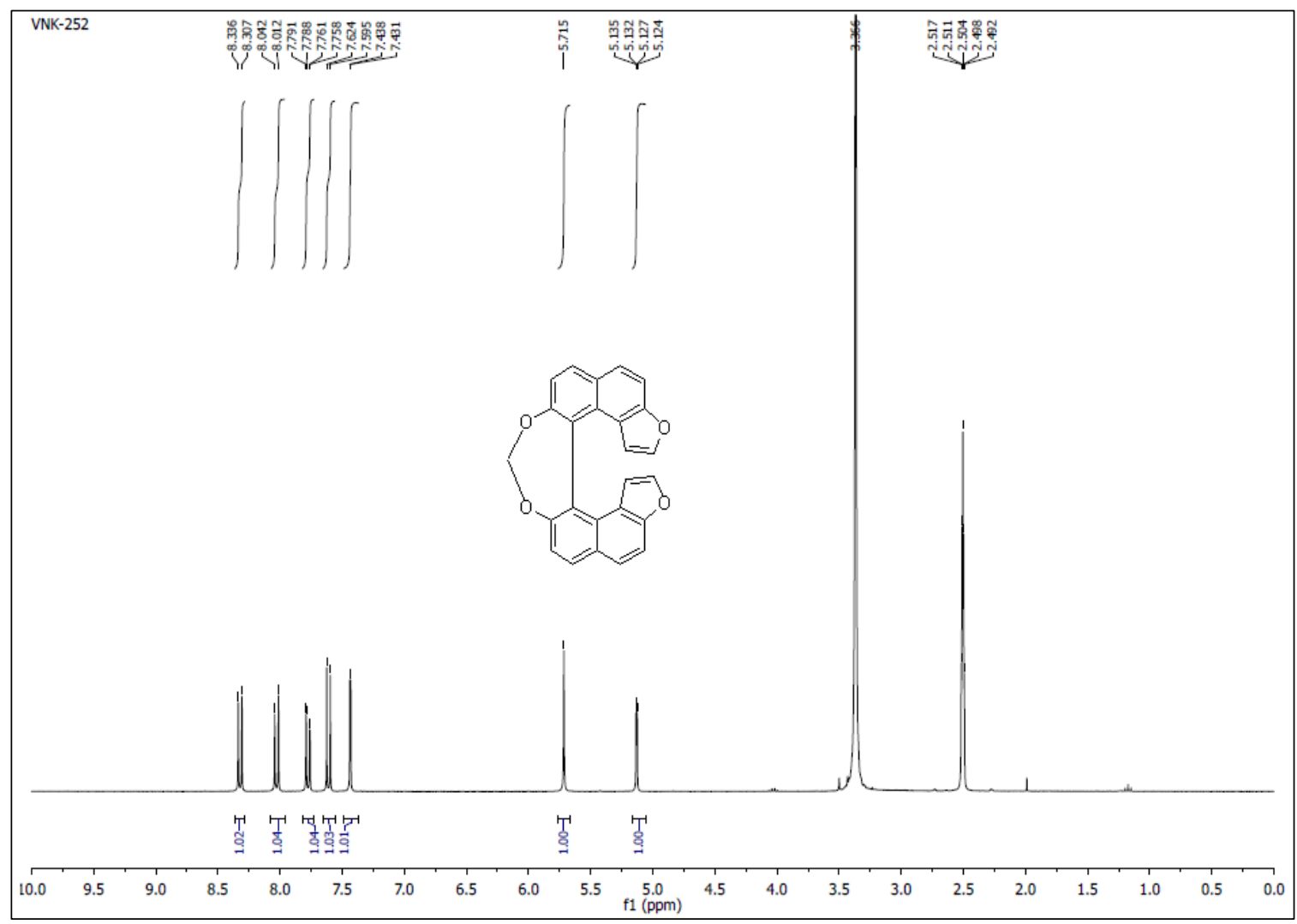

Figure S2. ${ }^{1} \mathrm{H}$ NMR $\left(300 \mathrm{MHz}, \mathrm{DMSO}-\mathrm{d}_{6}, \mathrm{ppm}\right)$ of helicenoid, 2. 


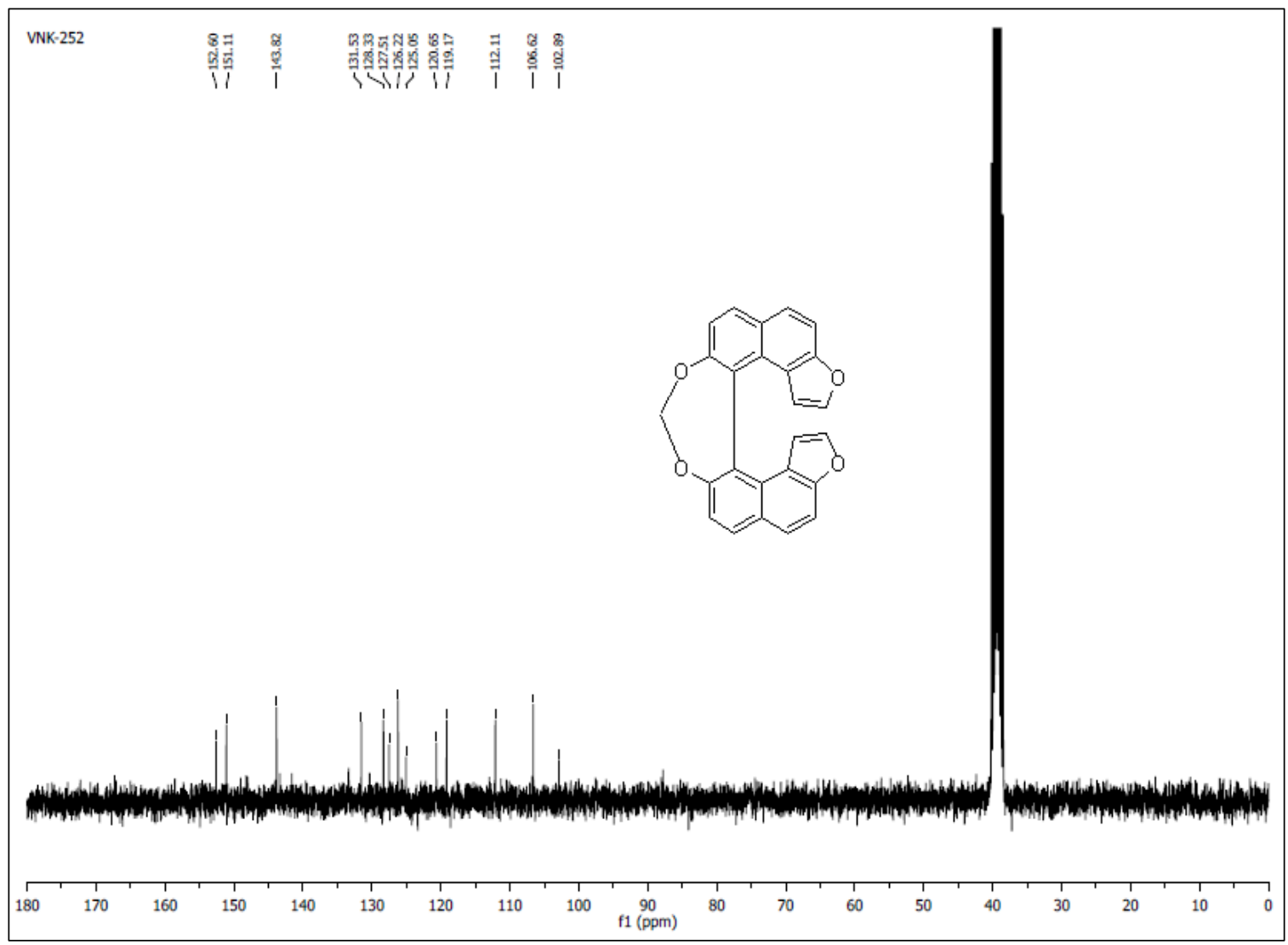

Figure S3. ${ }^{13} \mathrm{C}\left\{{ }^{1} \mathrm{H}\right\}$ NMR (75 MHz, DMSO-d 6 , ppm) of helicenoid, 2.

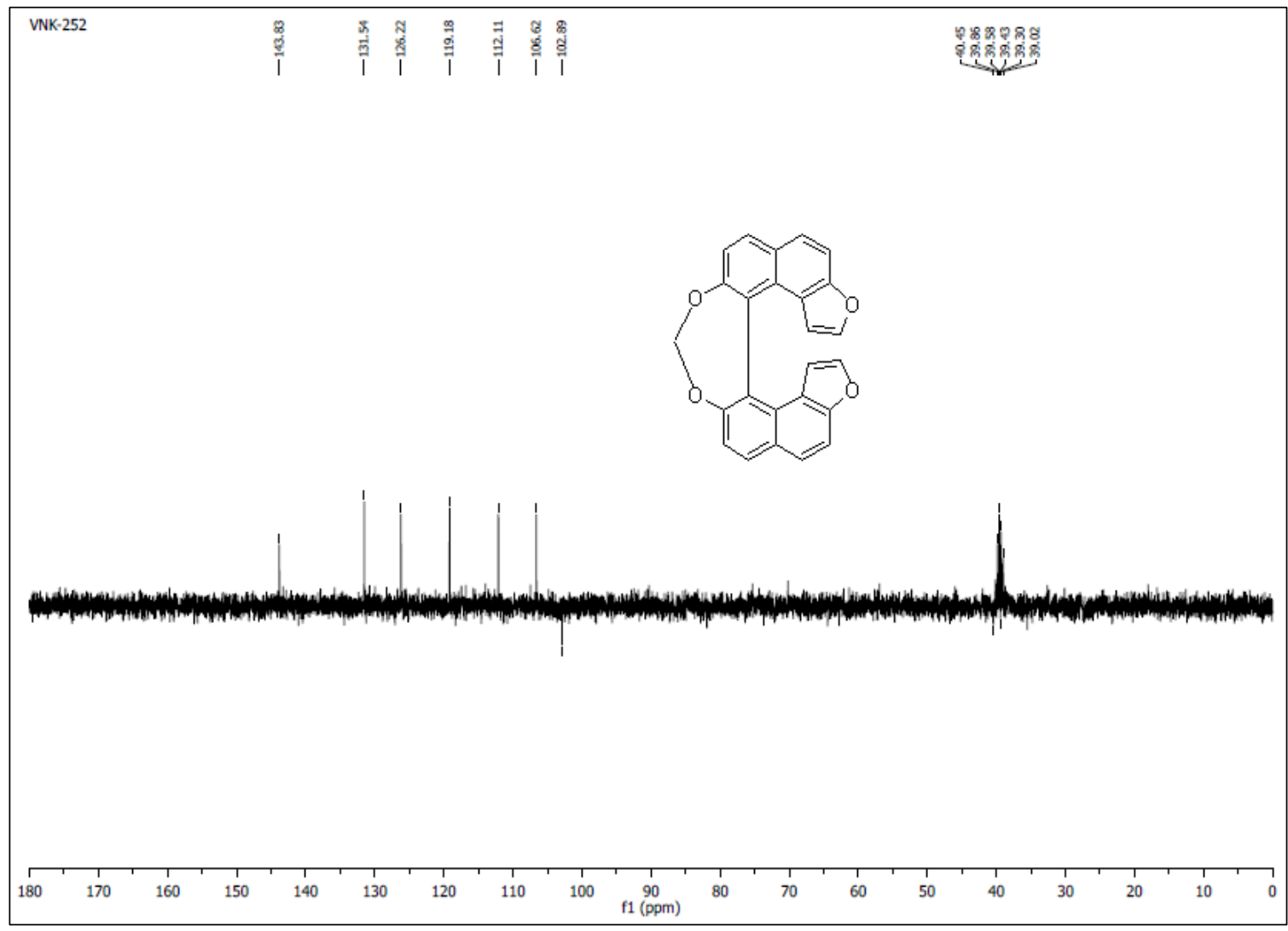

Figure S4. DEPT-135 NMR (75 MHz, DMSO-d 6 , ppm) of helicenoid, 2. 


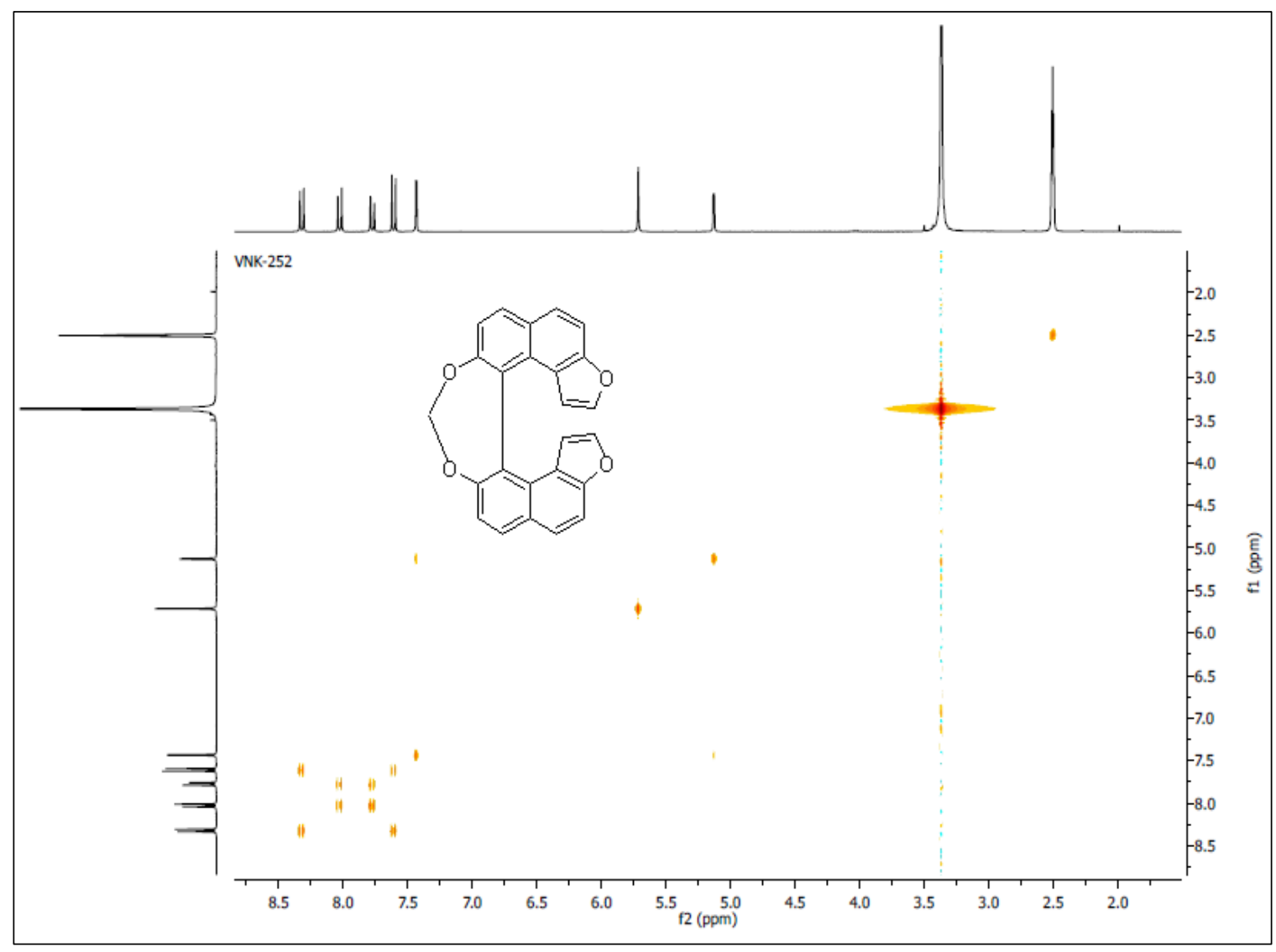

Figure S5. ${ }^{1} \mathrm{H}^{-1} \mathrm{H}$ COSY NMR (300 MHz, DMSO-d 6 , ppm) of helicenoid, 2.

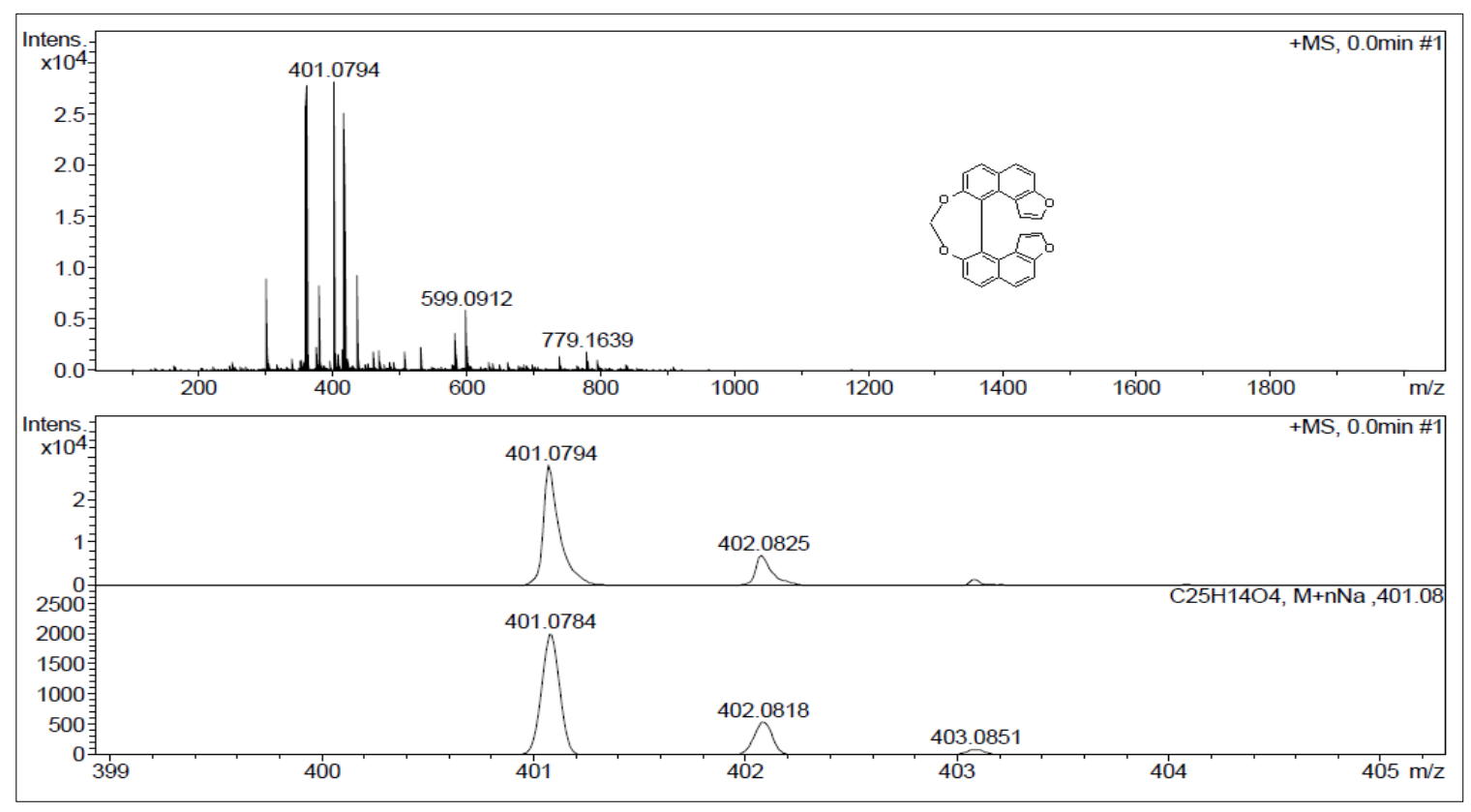

Figure S6. HRMS of helicenoid, 2. 


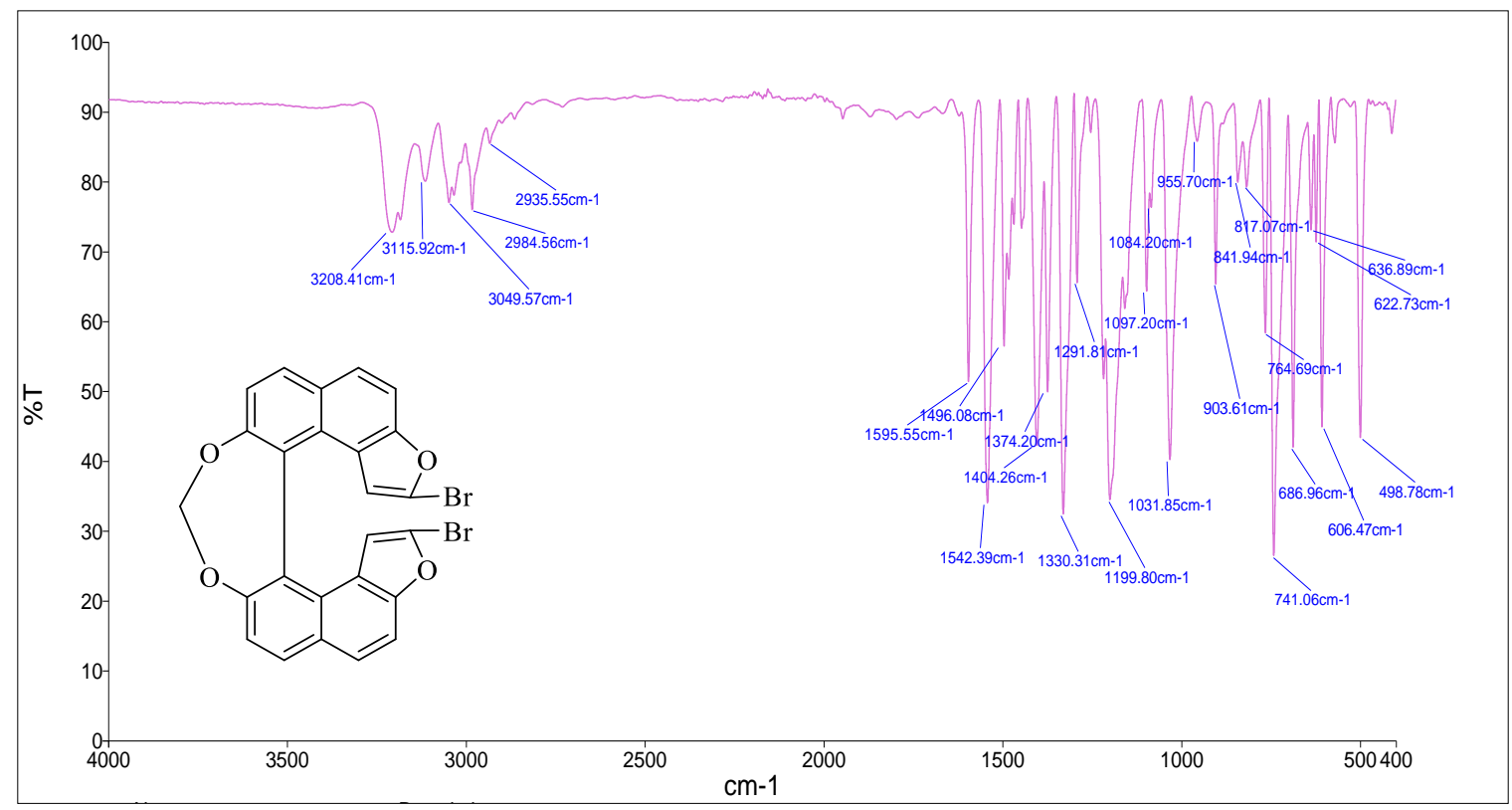

Figure S7. IR of di bromo helicenoid, 3.

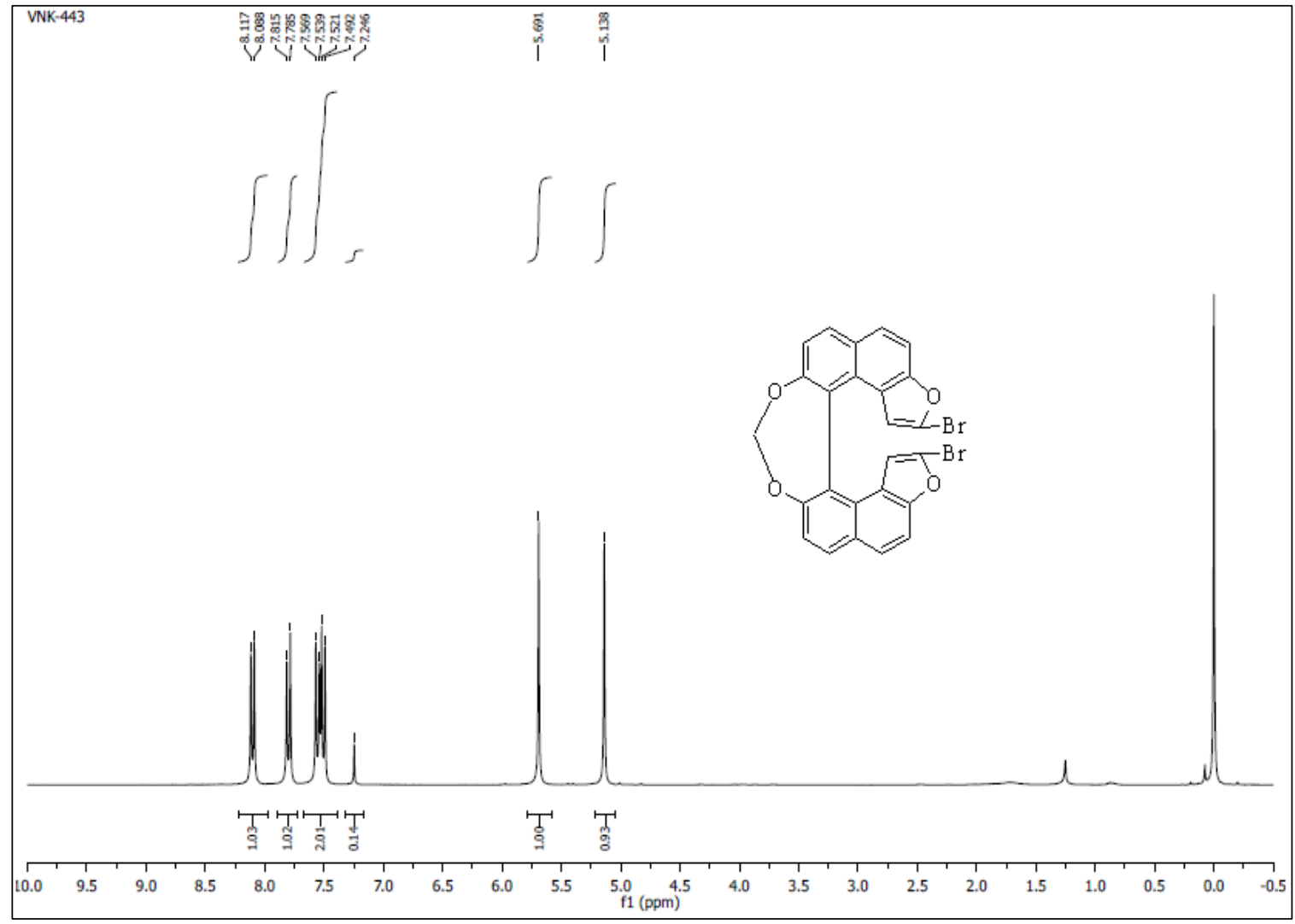

Figure S8. ${ }^{1} \mathrm{H}$ NMR $\left(300 \mathrm{MHz}, \mathrm{CDCl}_{3}, \mathrm{ppm}\right)$ of di bromo helicenoid, 3. 


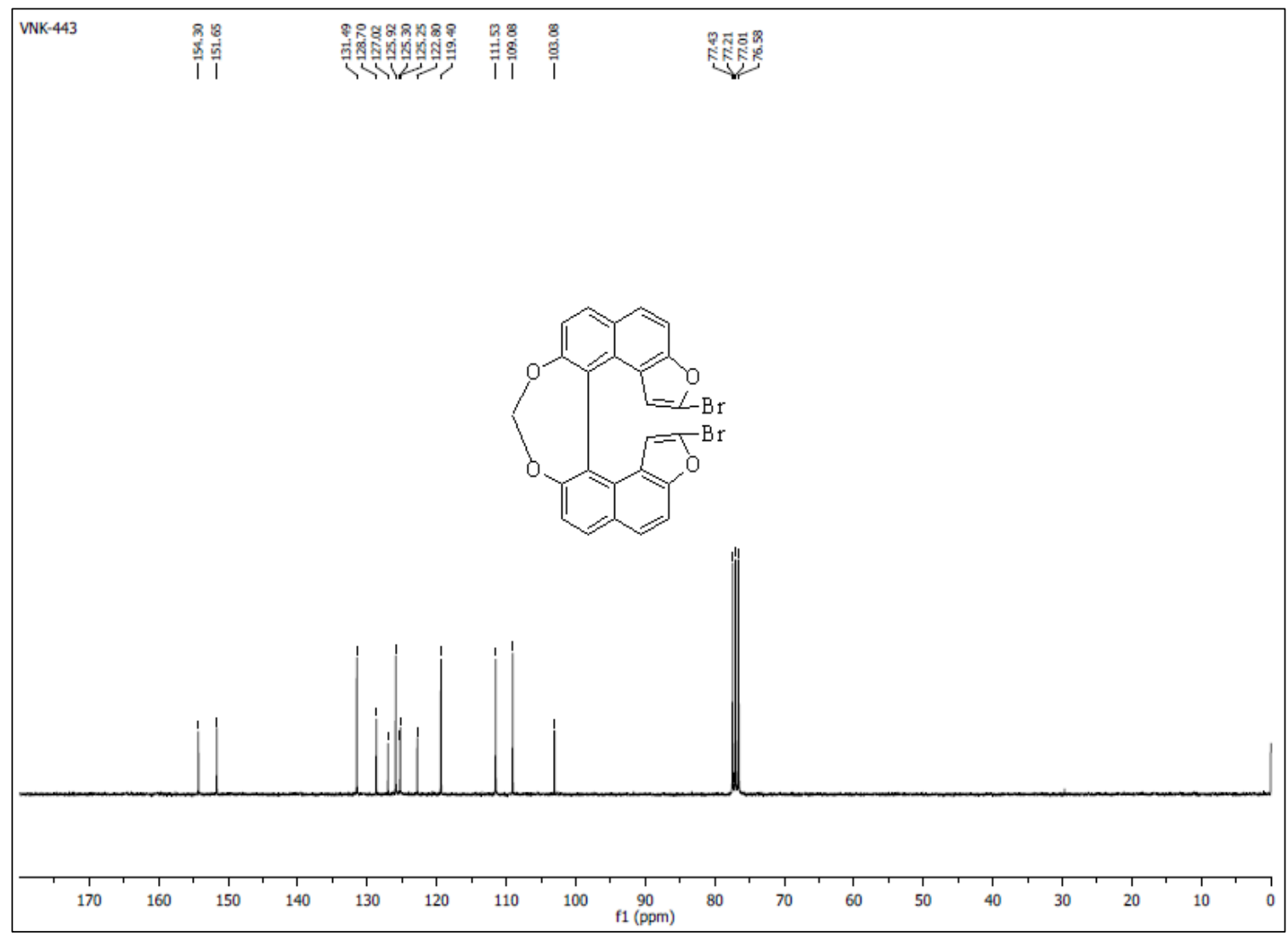

Figure S9. ${ }^{13} \mathrm{C}\left\{{ }^{1} \mathrm{H}\right\}$ NMR (75 MHz, $\left.\mathrm{CDCl}_{3}, \mathrm{ppm}\right)$ of di bromo Helicenoid, 3 .

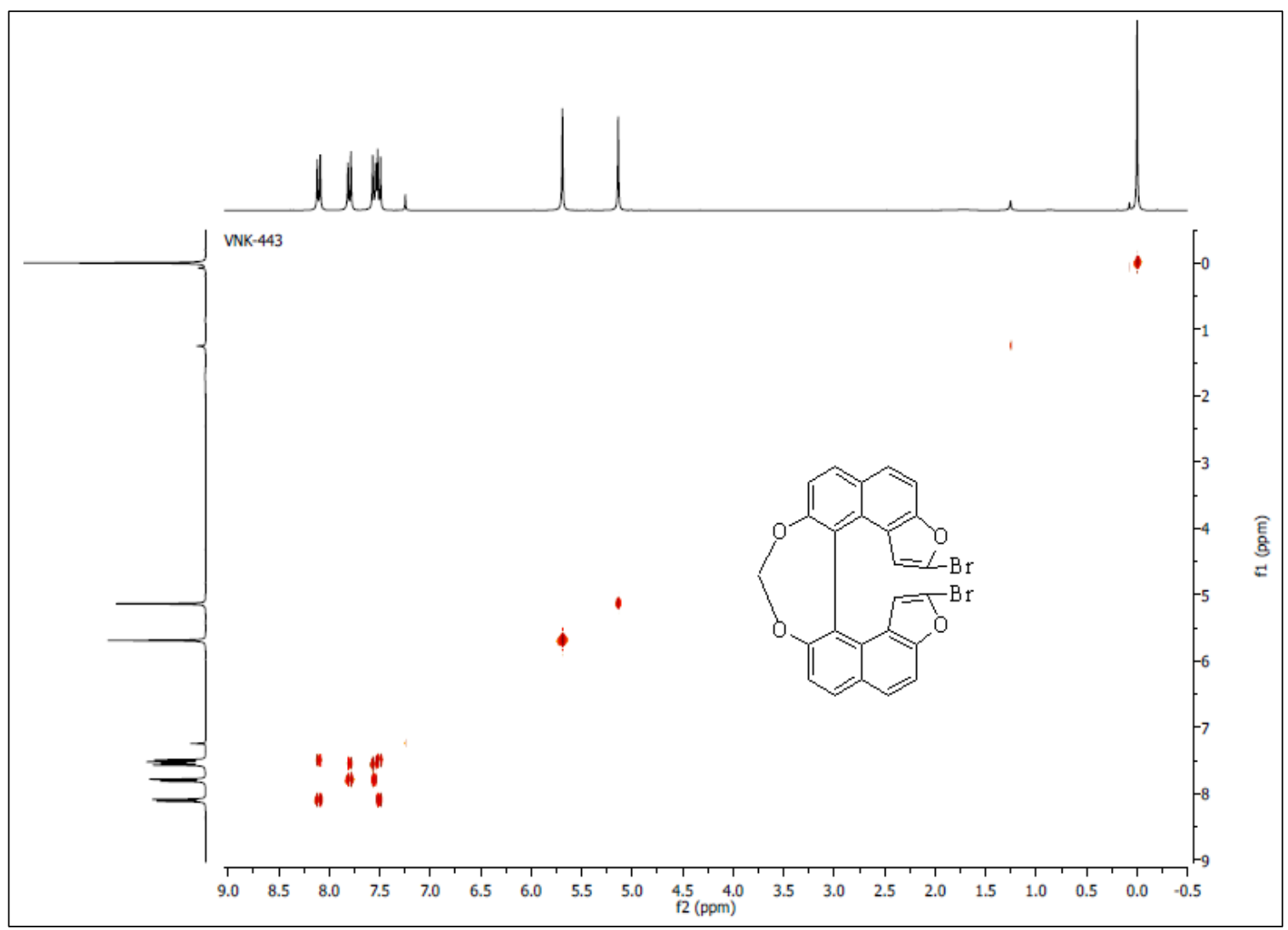

Figure S10. ${ }^{1} \mathrm{H}-{ }^{1} \mathrm{H} \operatorname{COSY}\left(300 \mathrm{MHz}, \mathrm{CDCl}_{3}, \mathrm{ppm}\right)$ of di bromo helicenoid, 3 . 


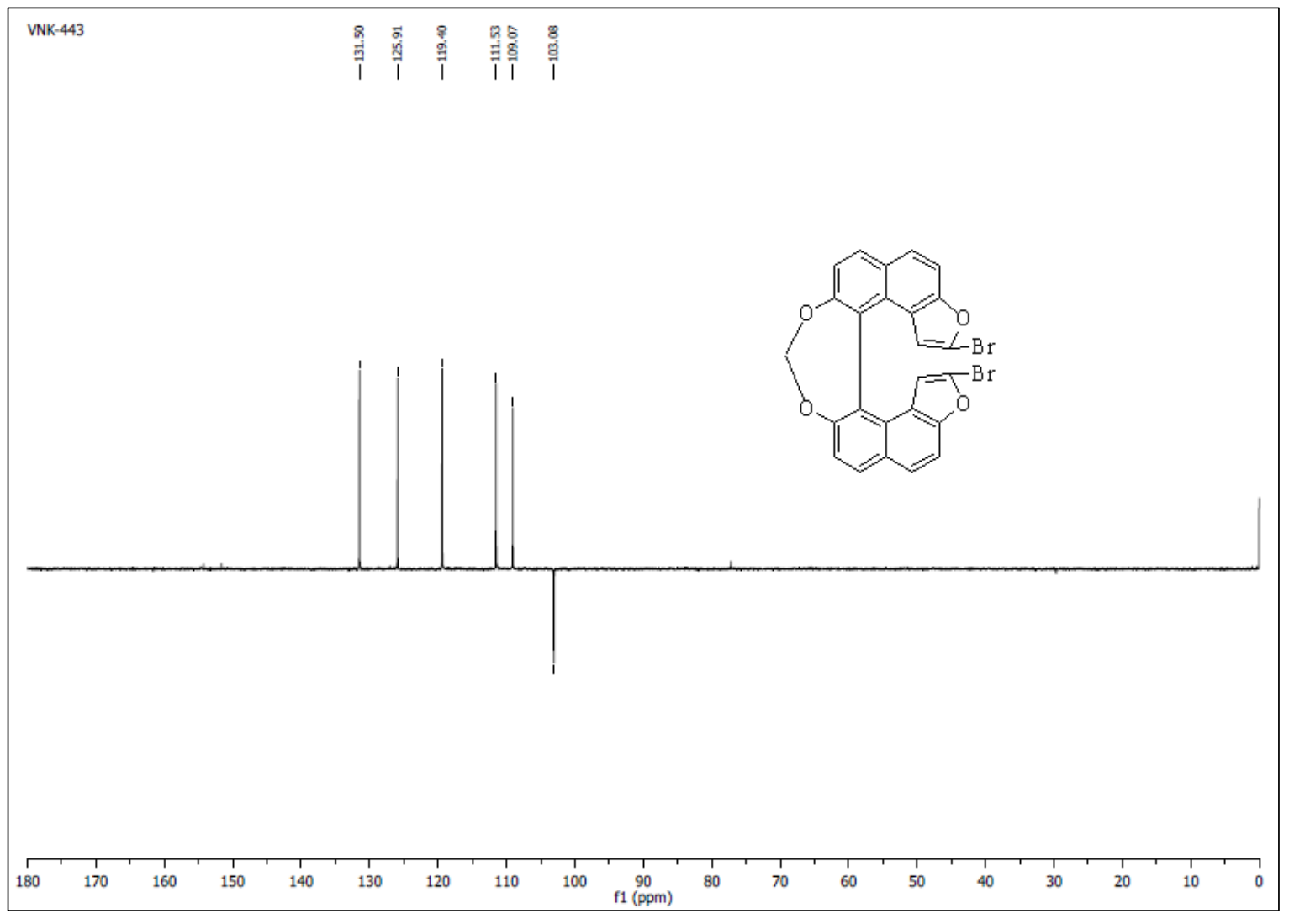

Figure S11. DEPT-135 NMR (75 MHz, $\left.\mathrm{CDCl}_{3}, \mathrm{ppm}\right)$ of di bromo helicenoid, 3.

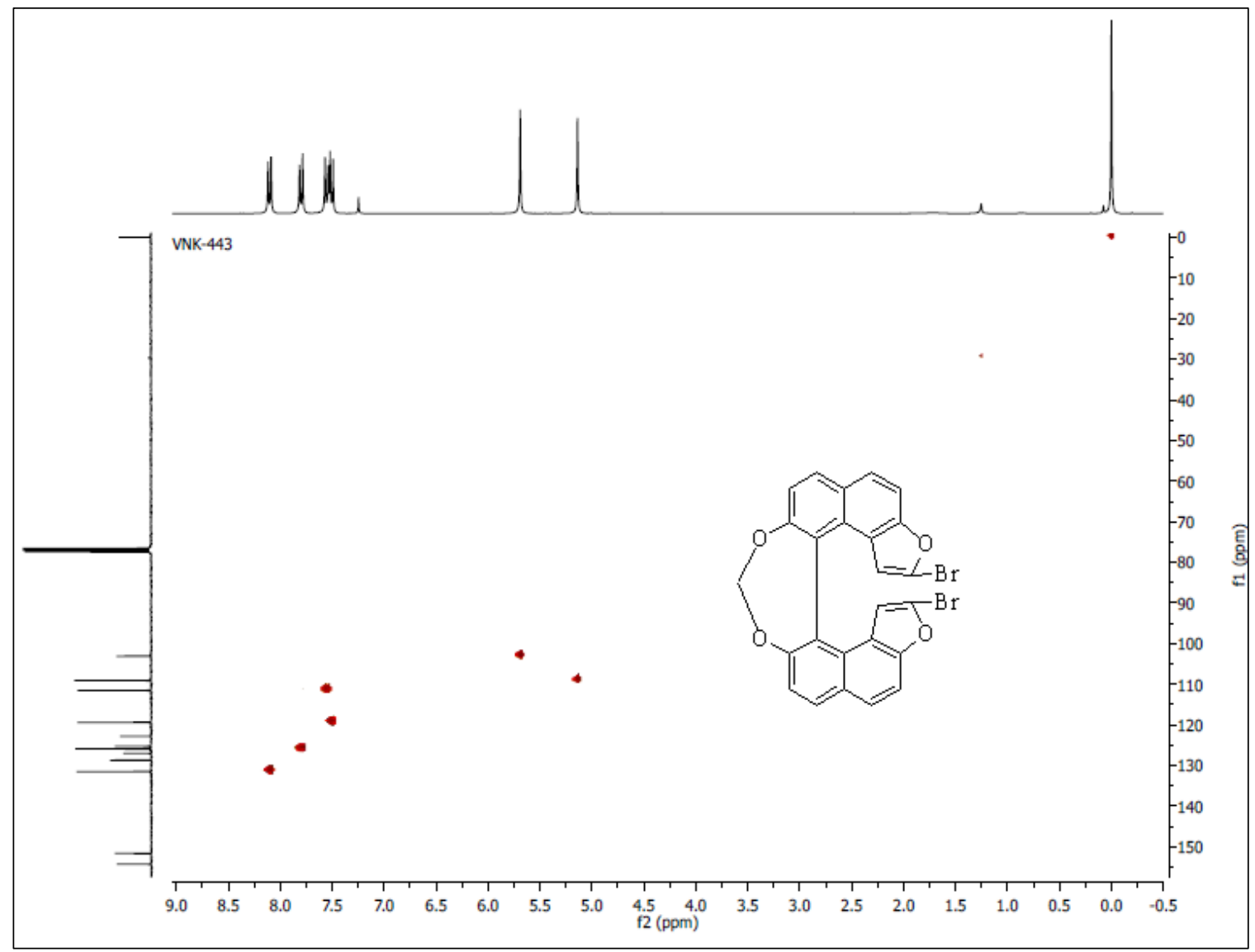

Figure S12. ${ }^{1} \mathrm{H}^{-13} \mathrm{C} \mathrm{HSQC}\left(300 \mathrm{MHZ}\right.$ and $\left.75 \mathrm{MHz}, \mathrm{CDCl}_{3}, \mathrm{ppm}\right)$ of di bromo helicenoid, 3. 


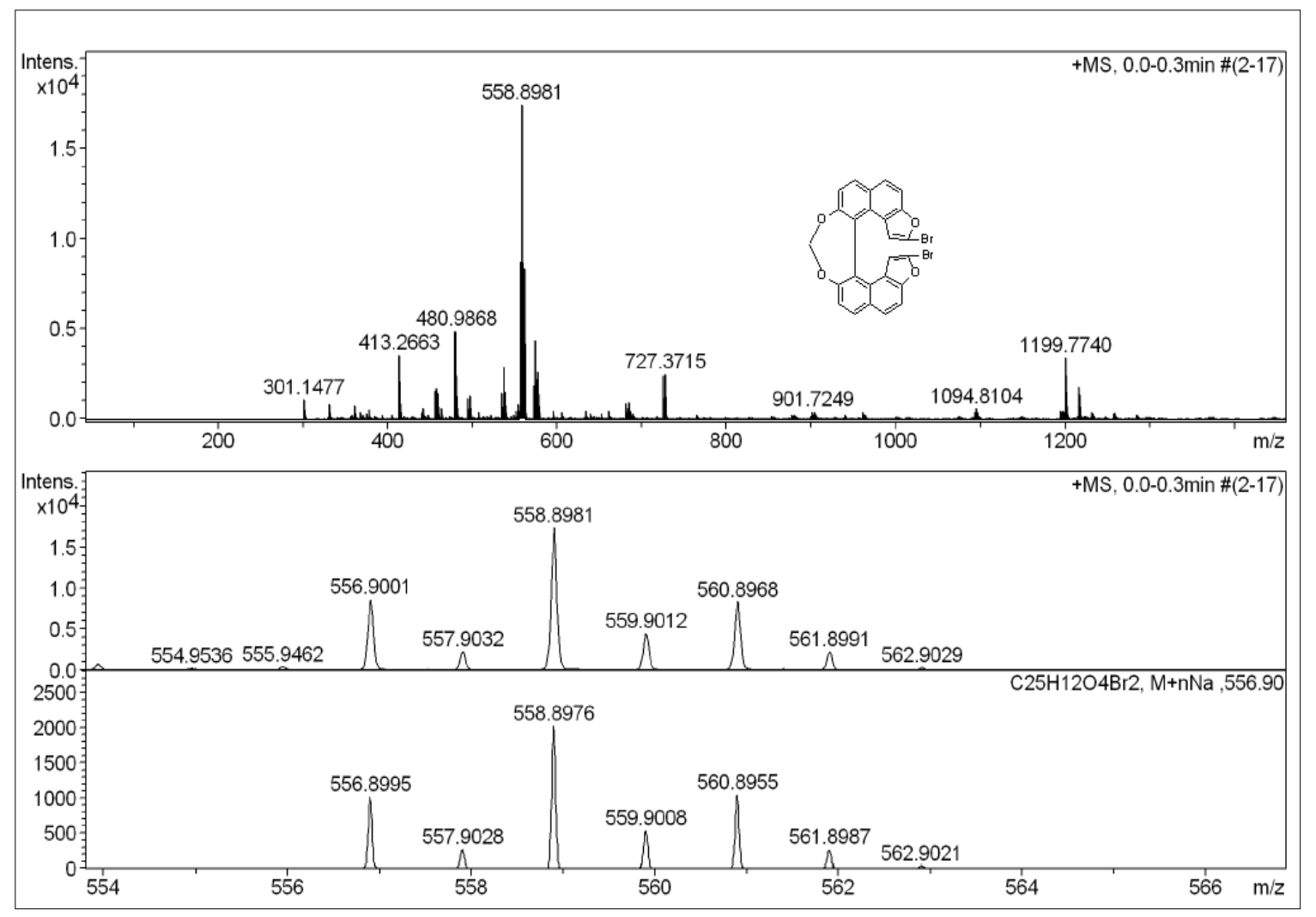

Figure S13. HRMS of di bromo helicenoid, 3.

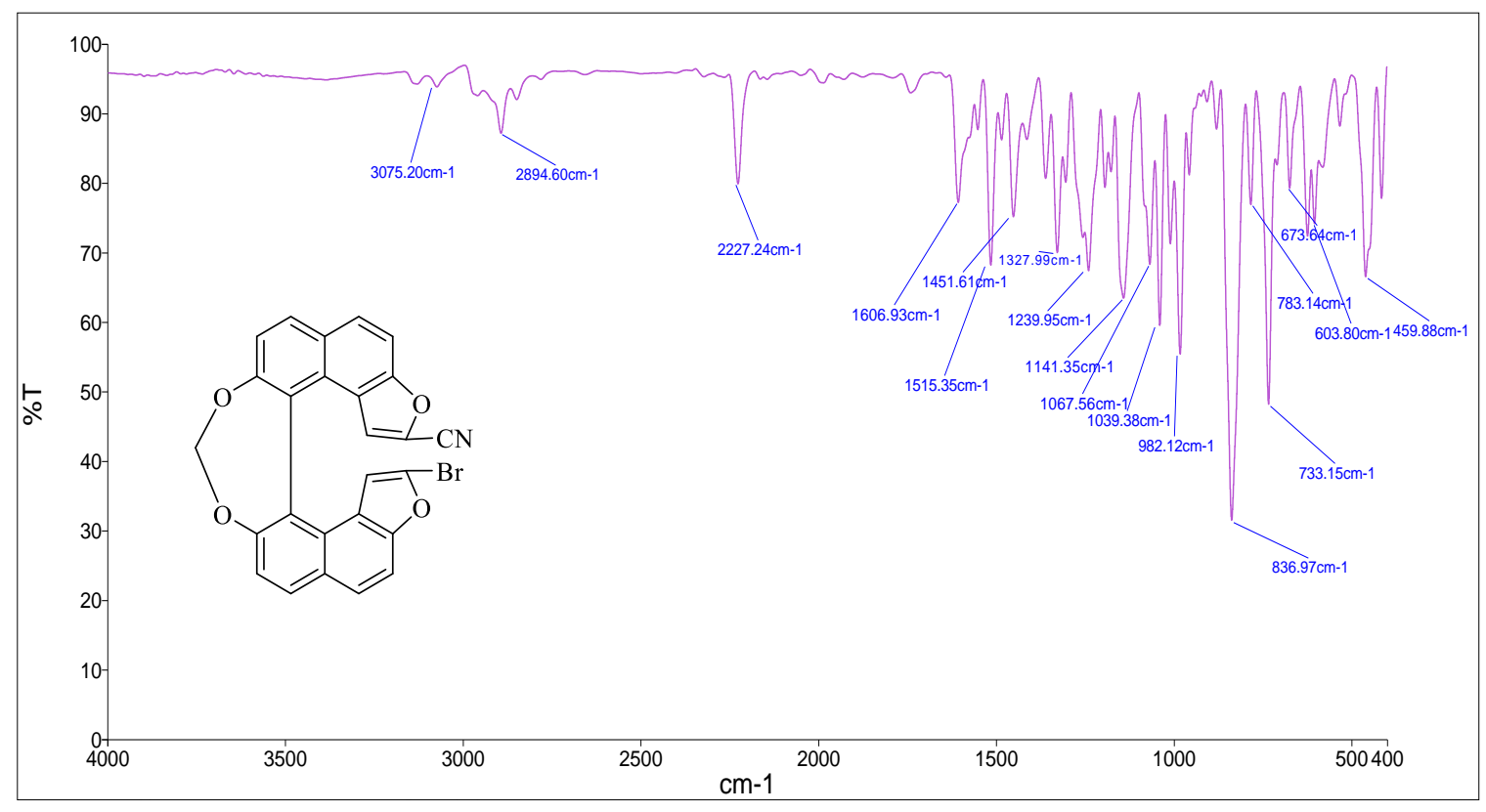

Figure S14. IR of helicenoid mono bromo cyanide, 4. 


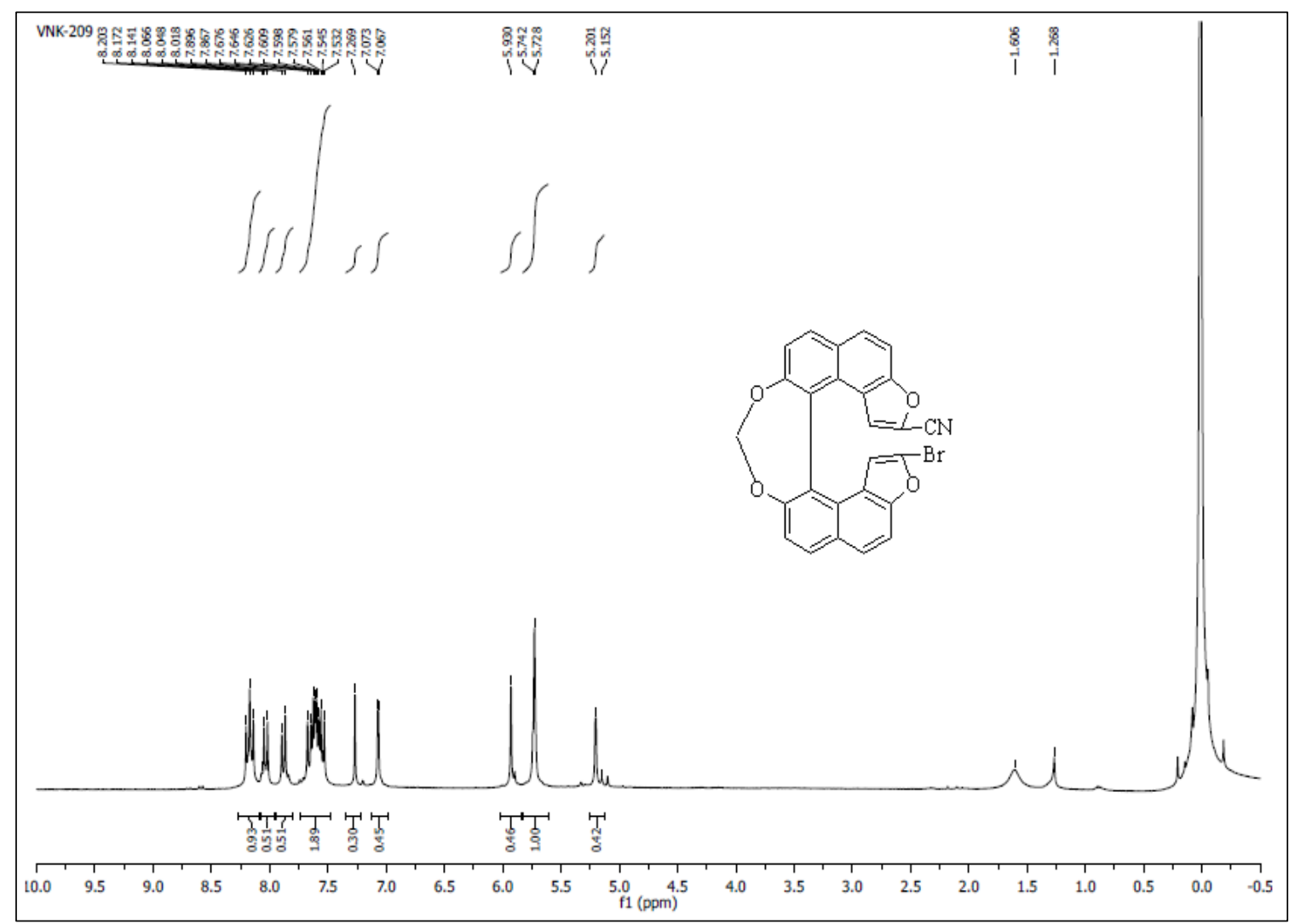

Figure S15. ${ }^{1} \mathrm{H}$ NMR $\left(300 \mathrm{MHz}, \mathrm{CDCl}_{3}, \mathrm{ppm}\right)$ of helicenoid mono bromo cyanide, 4.

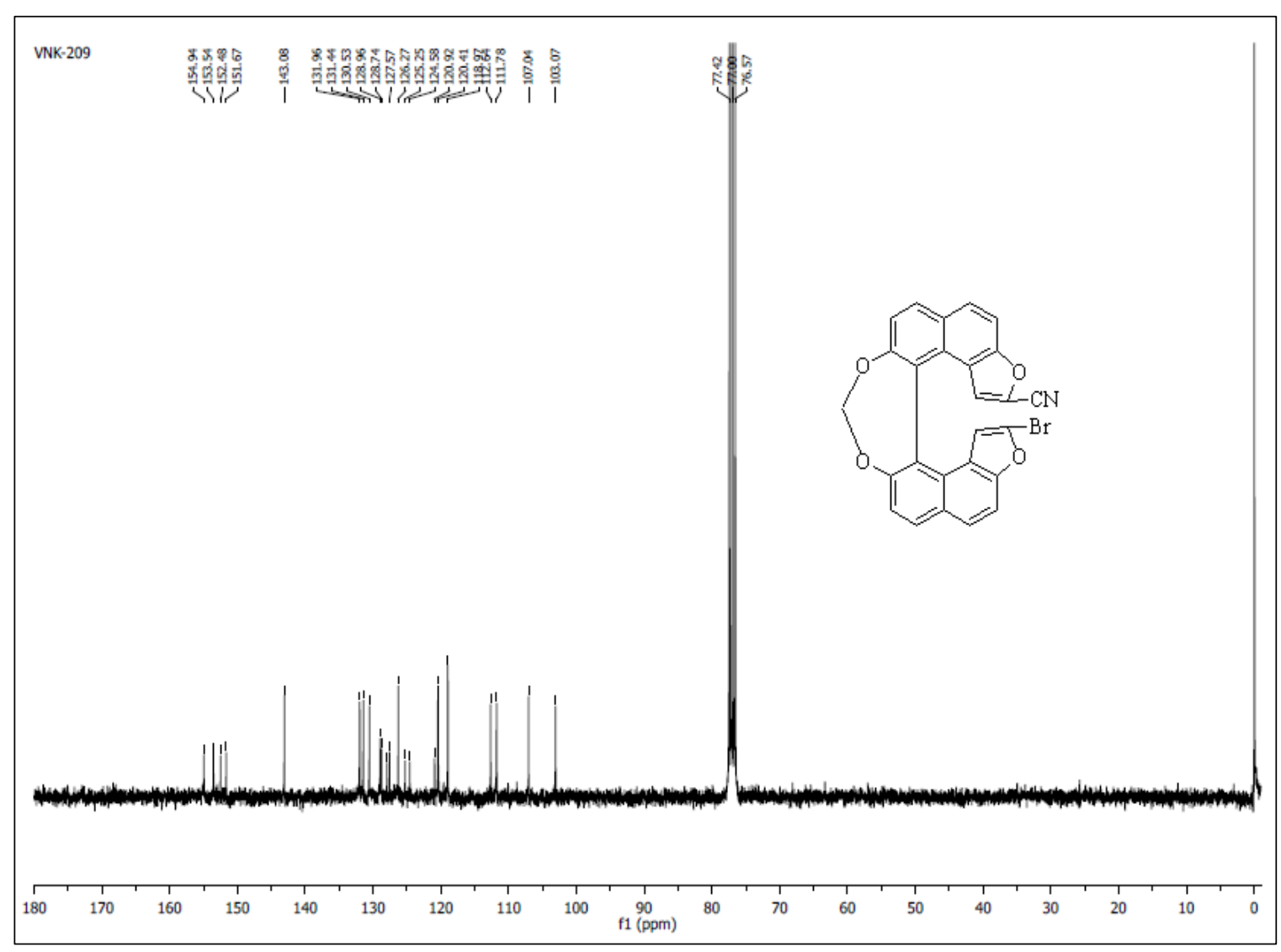

Figure S16. ${ }^{13} \mathrm{C}\left\{{ }^{1} \mathrm{H}\right\}$ NMR $\left(75 \mathrm{MHz}, \mathrm{CDCl}_{3}, \mathrm{ppm}\right)$ of helicenoid mono bromo cyanide, 4 . 


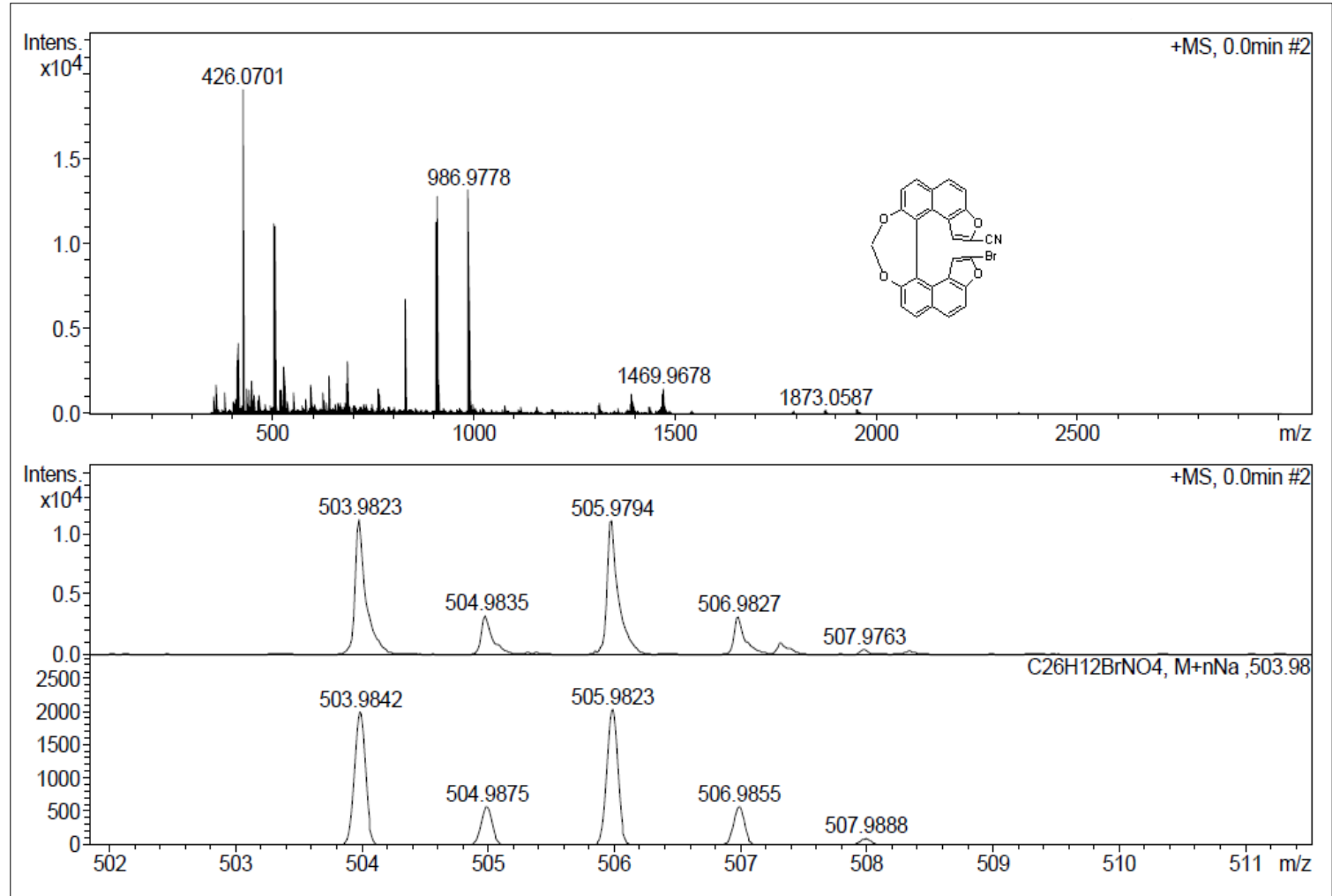

Figure S17. HRMS of helicenoid mono bromo cyanide, 4.

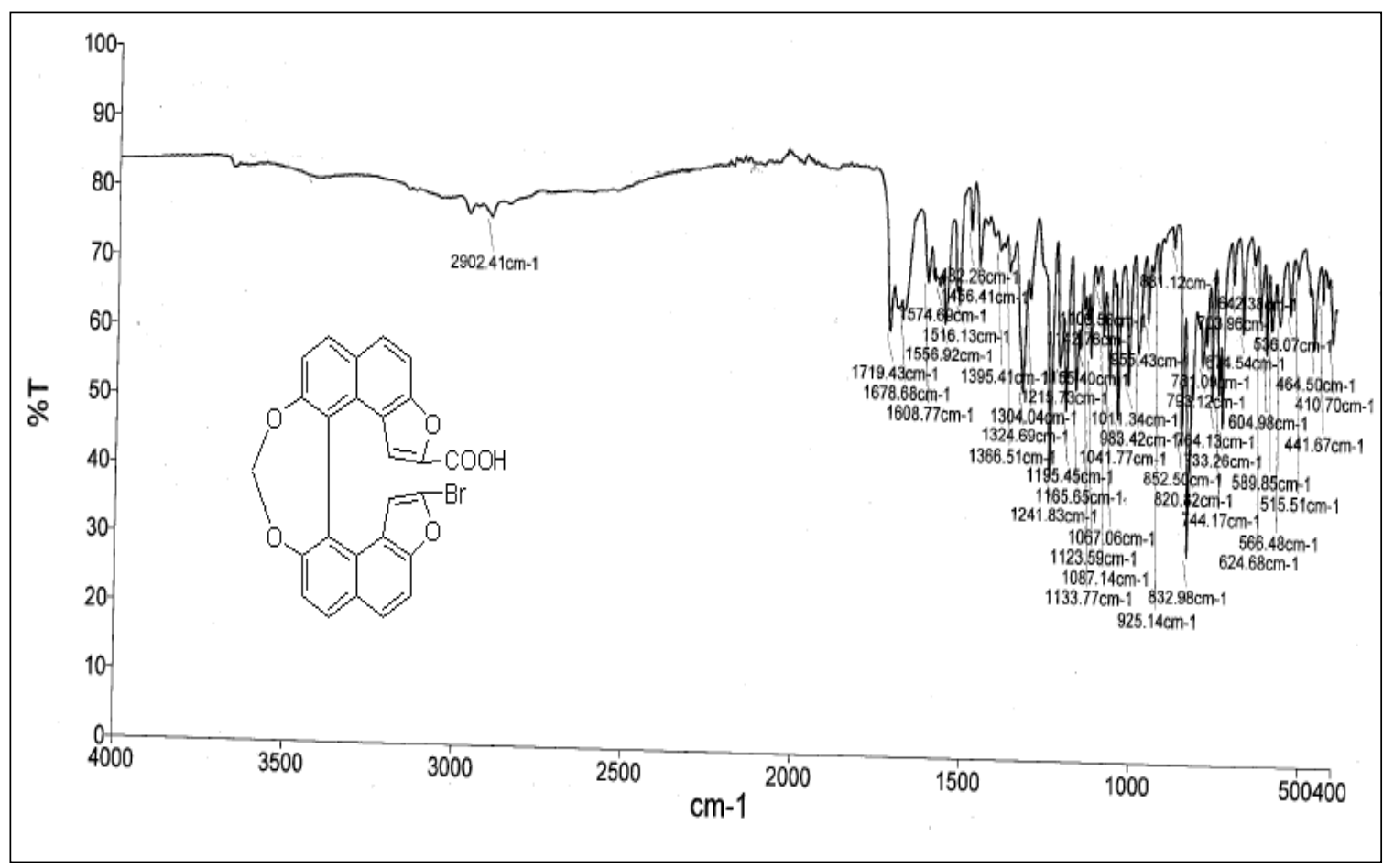

Figure S18. IR of helicenoid mono bromo carboxylic acid, 5. 


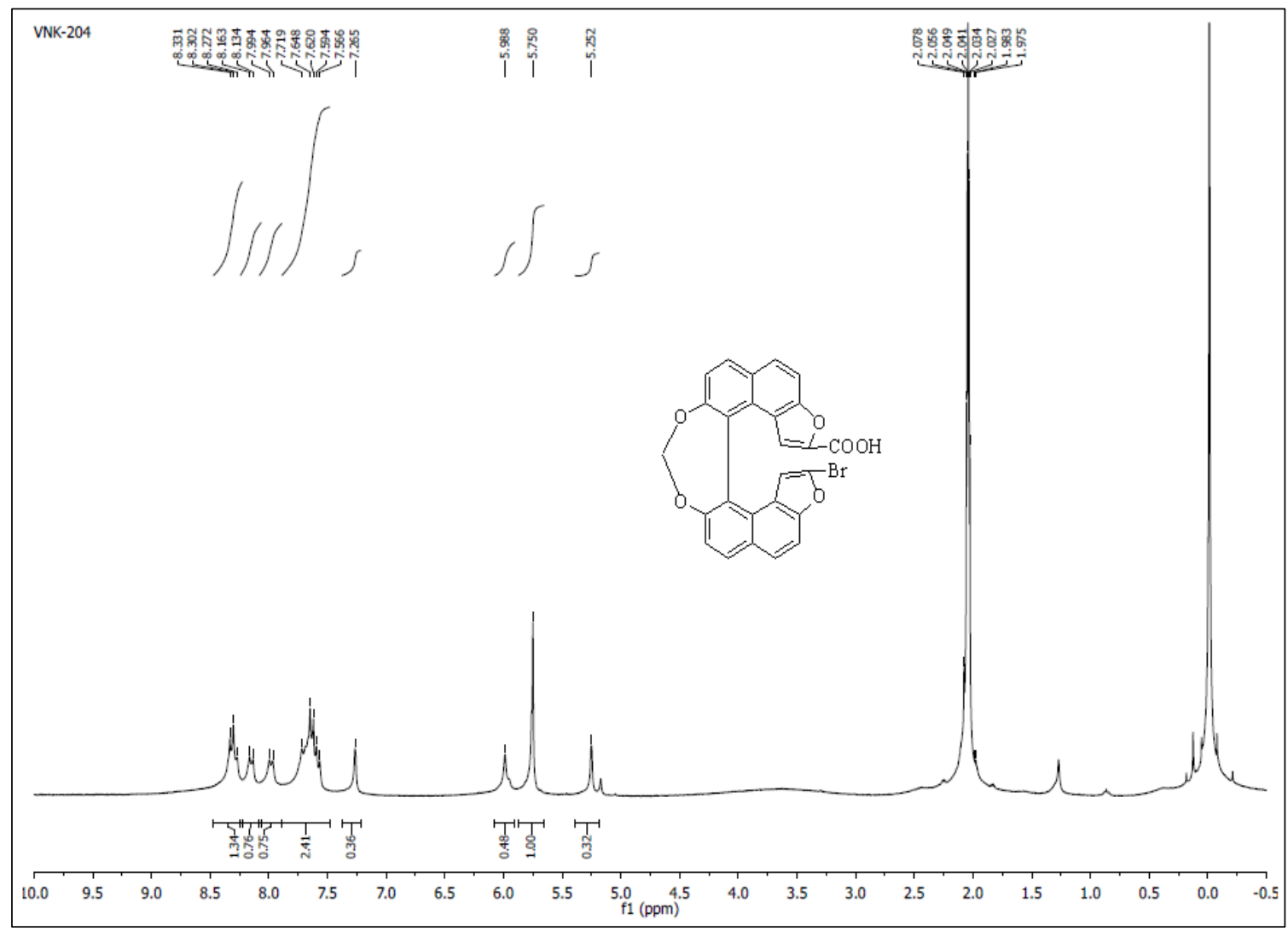

Figure S19. ${ }^{1} \mathrm{H}$ NMR $\left(300 \mathrm{MHz},\left(\mathrm{CD}_{3}\right)_{2} \mathrm{CO}\right.$, ppm) of helicenoid mono bromo carboxylic acid, 5.

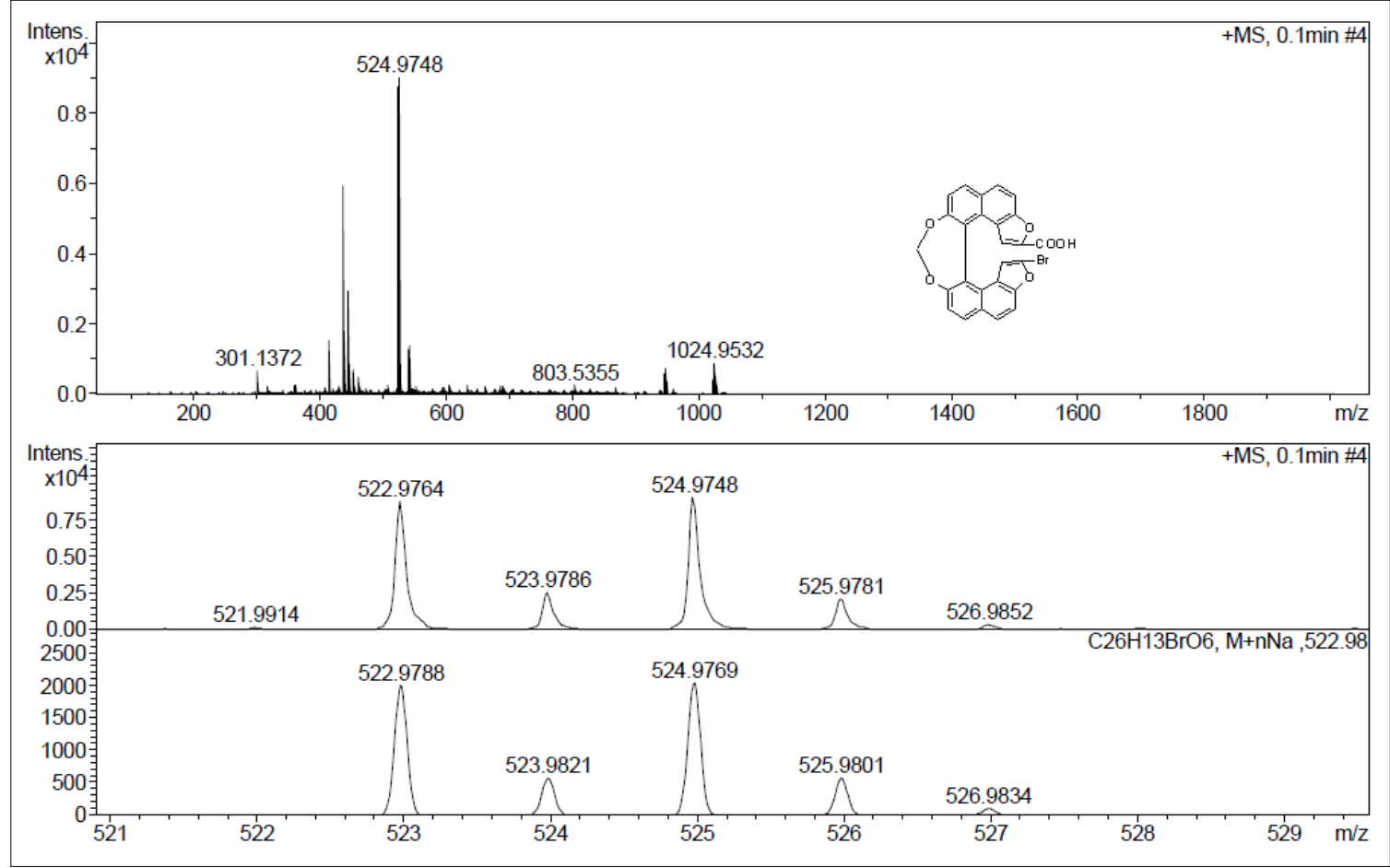

Figure S20. HRMS of helicenoid mono bromo carboxylic acid, 5. 


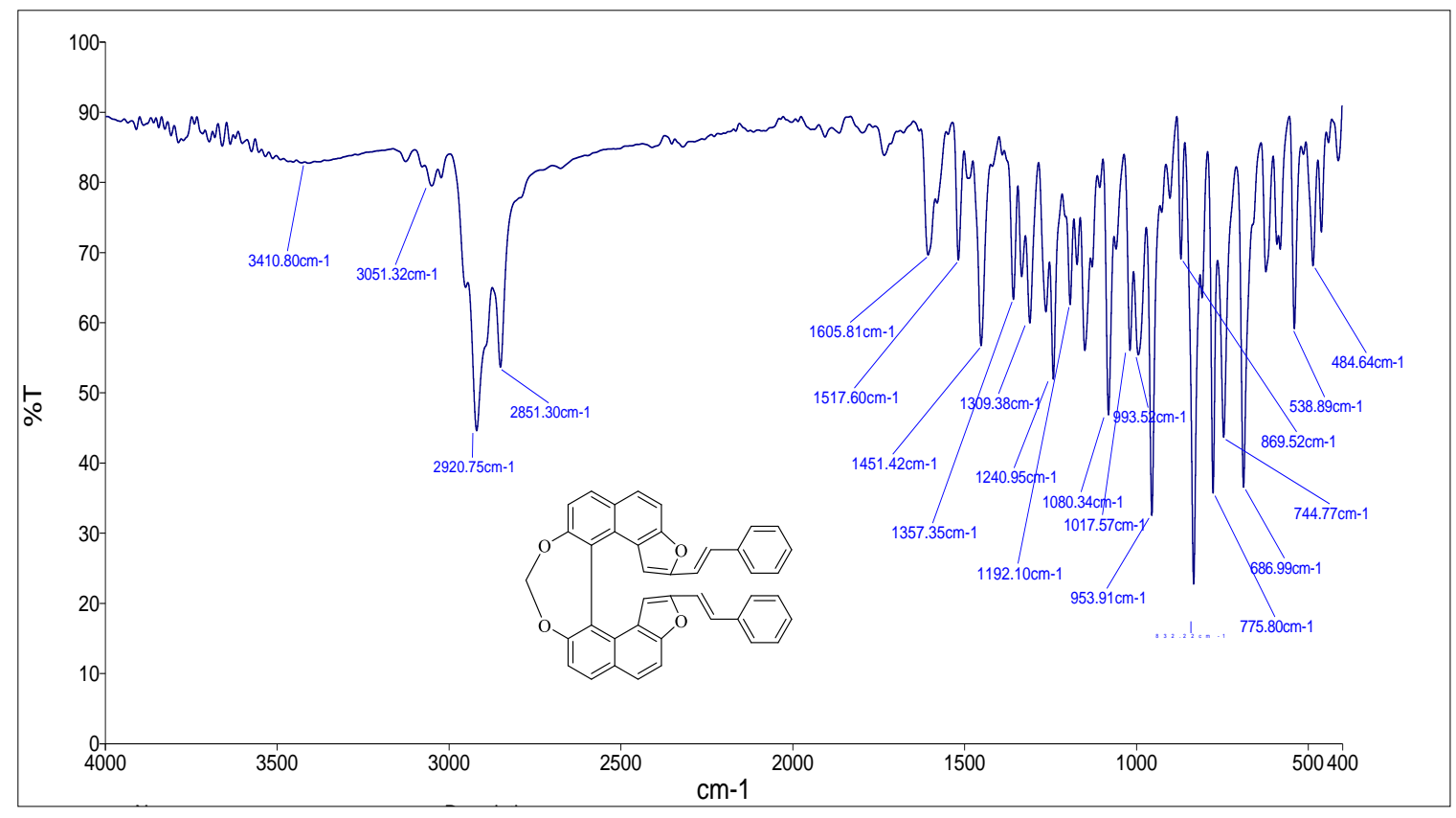

Figure S21. IR of 2,16-distyryl helicenoid, 6.

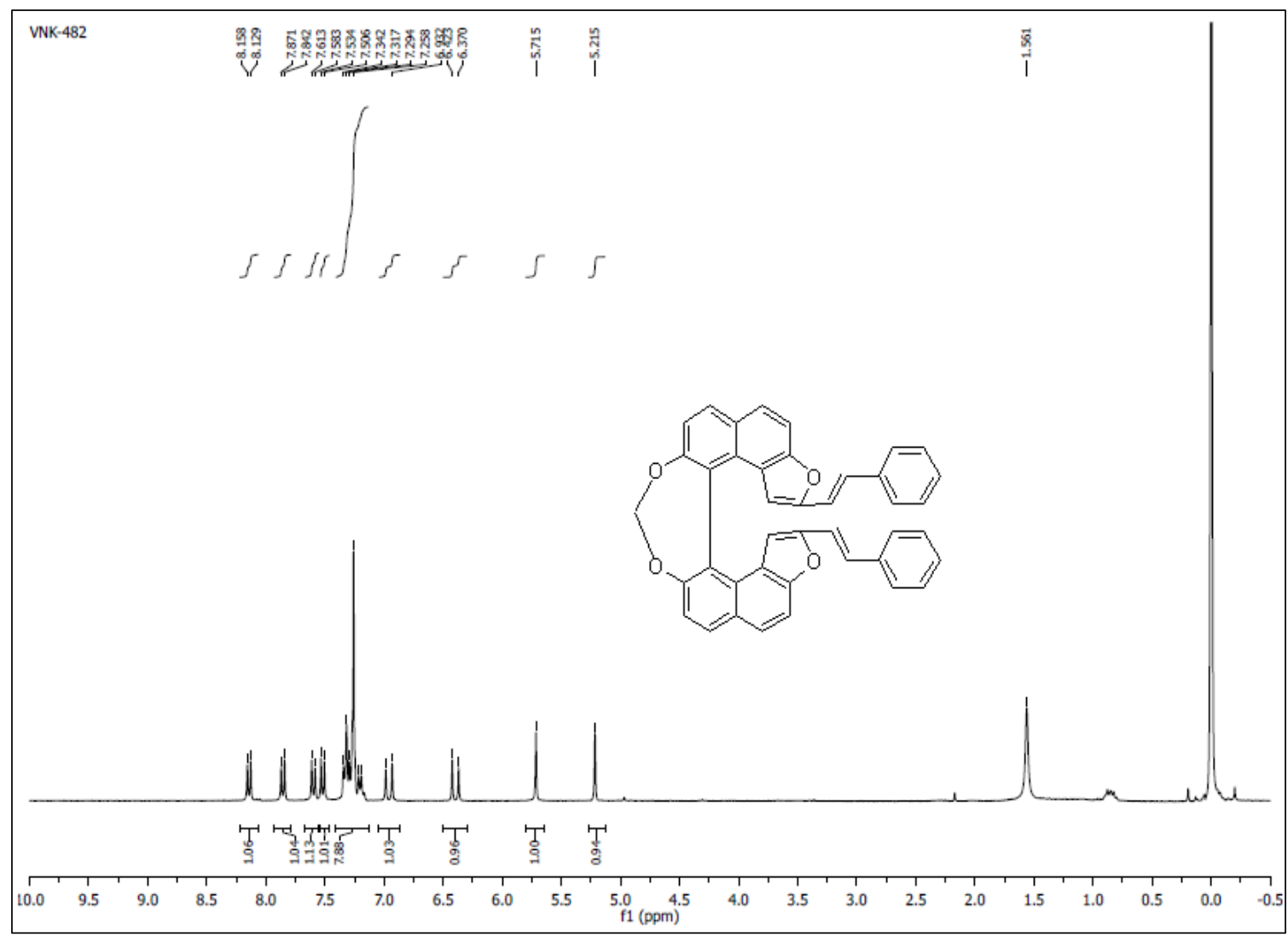

Figure S22. ${ }^{1} \mathrm{H} \mathrm{NMR}\left(300 \mathrm{MHz}, \mathrm{CDCl}_{3}\right.$, ppm) 2,16-distyryl helicenoid, 6. 


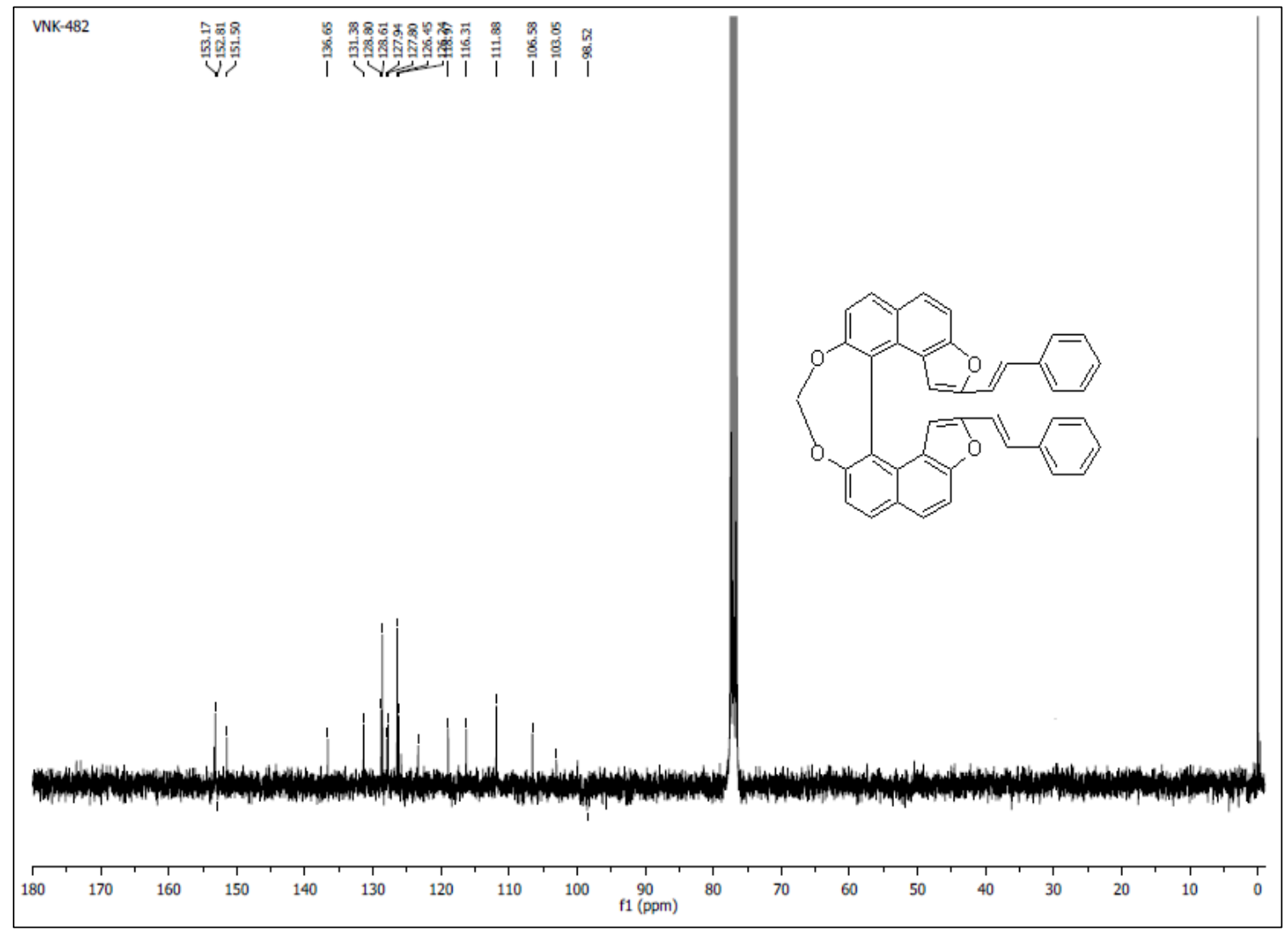

Figure S23. ${ }^{13} \mathrm{C}\left\{{ }^{1} \mathrm{H}\right\}$ NMR (75 MHz, $\left.\mathrm{CDCl}_{3}, \mathrm{ppm}\right)$ 2,16'-distyryl helicenoid, 6.

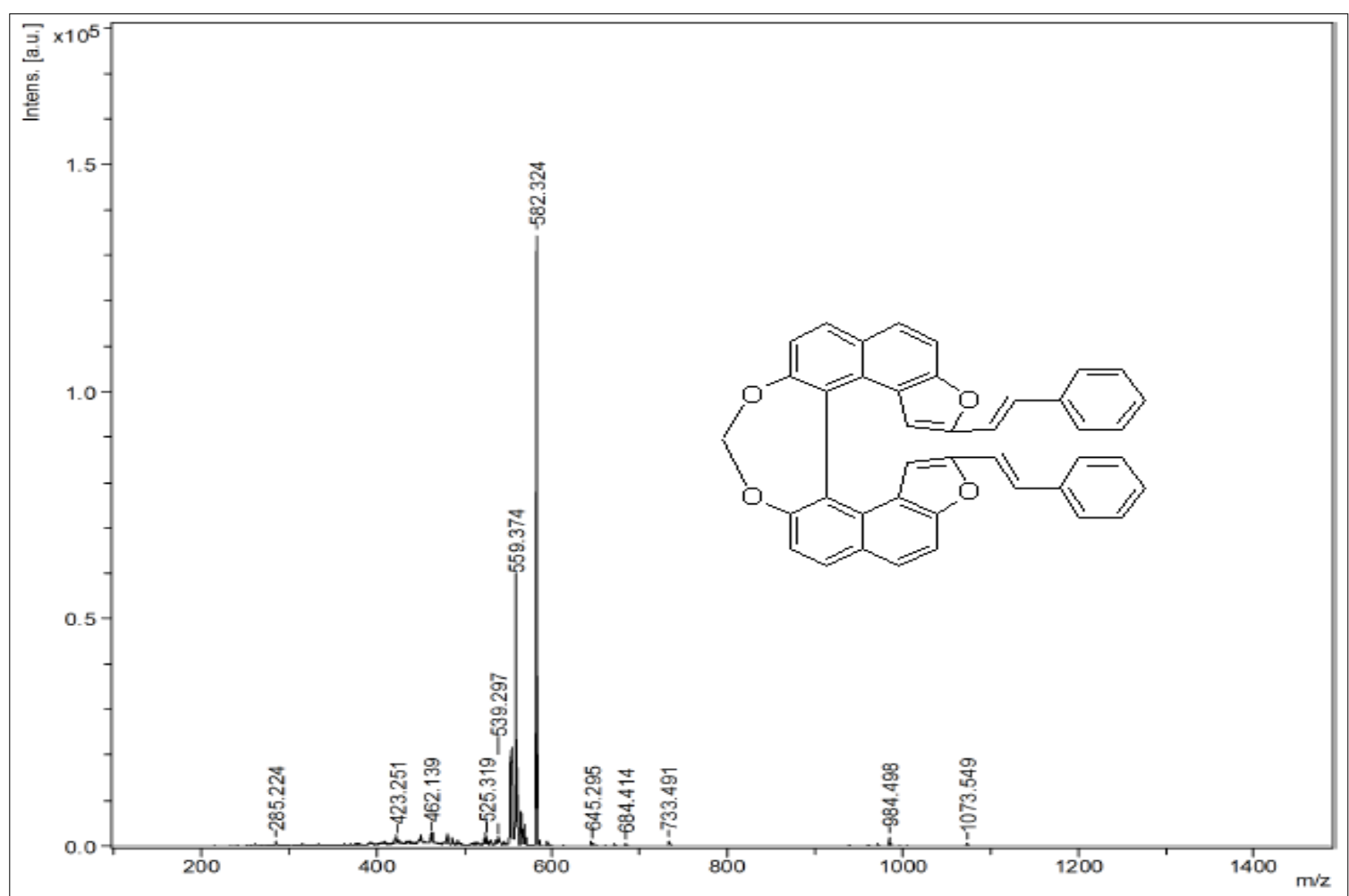

Figure S24. MALDI TOF mass of 2,16'-distyryl helicenoid, 6. 


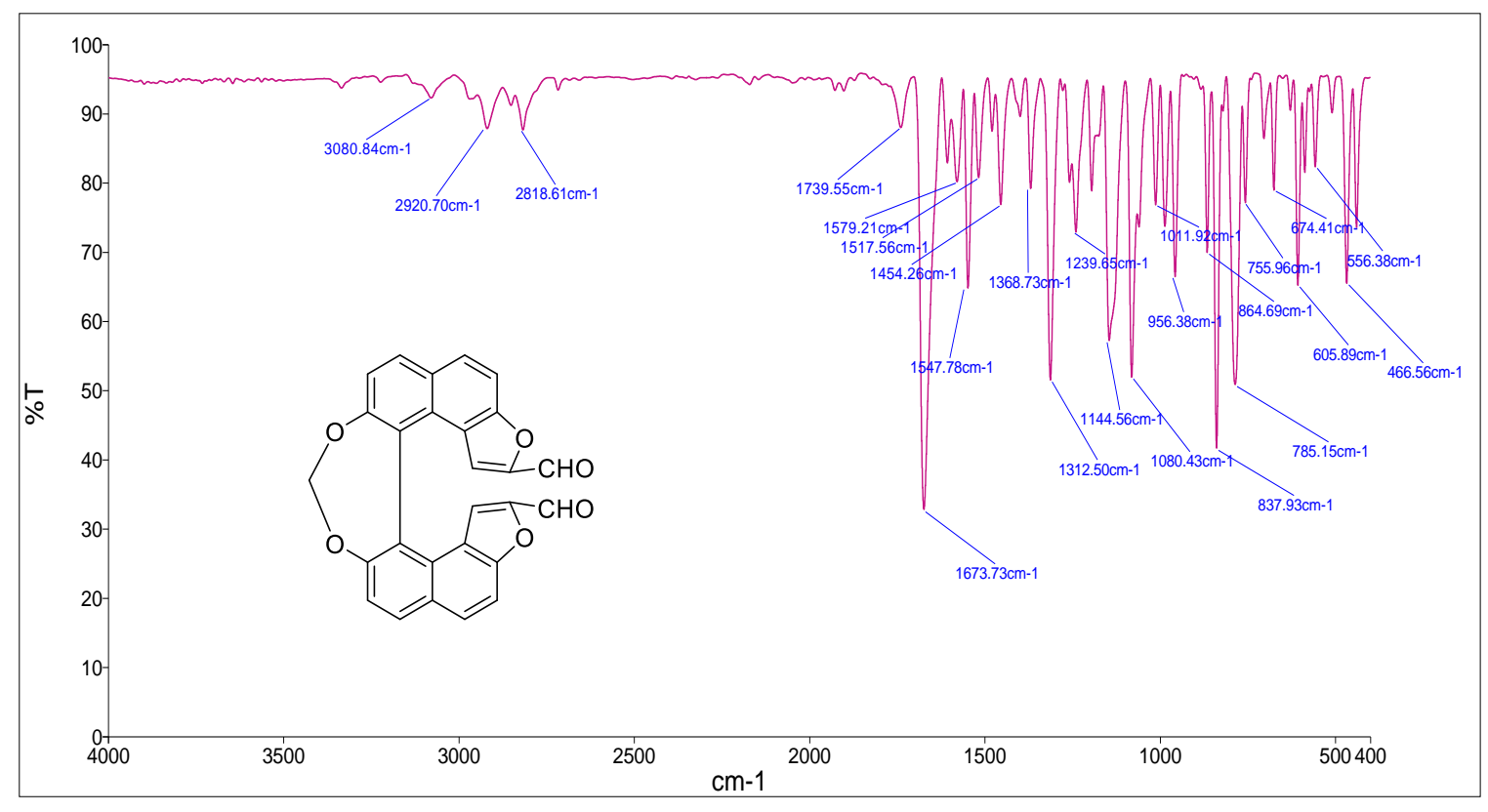

Figure S25. IR of helicenoid dialdehyde, 7.

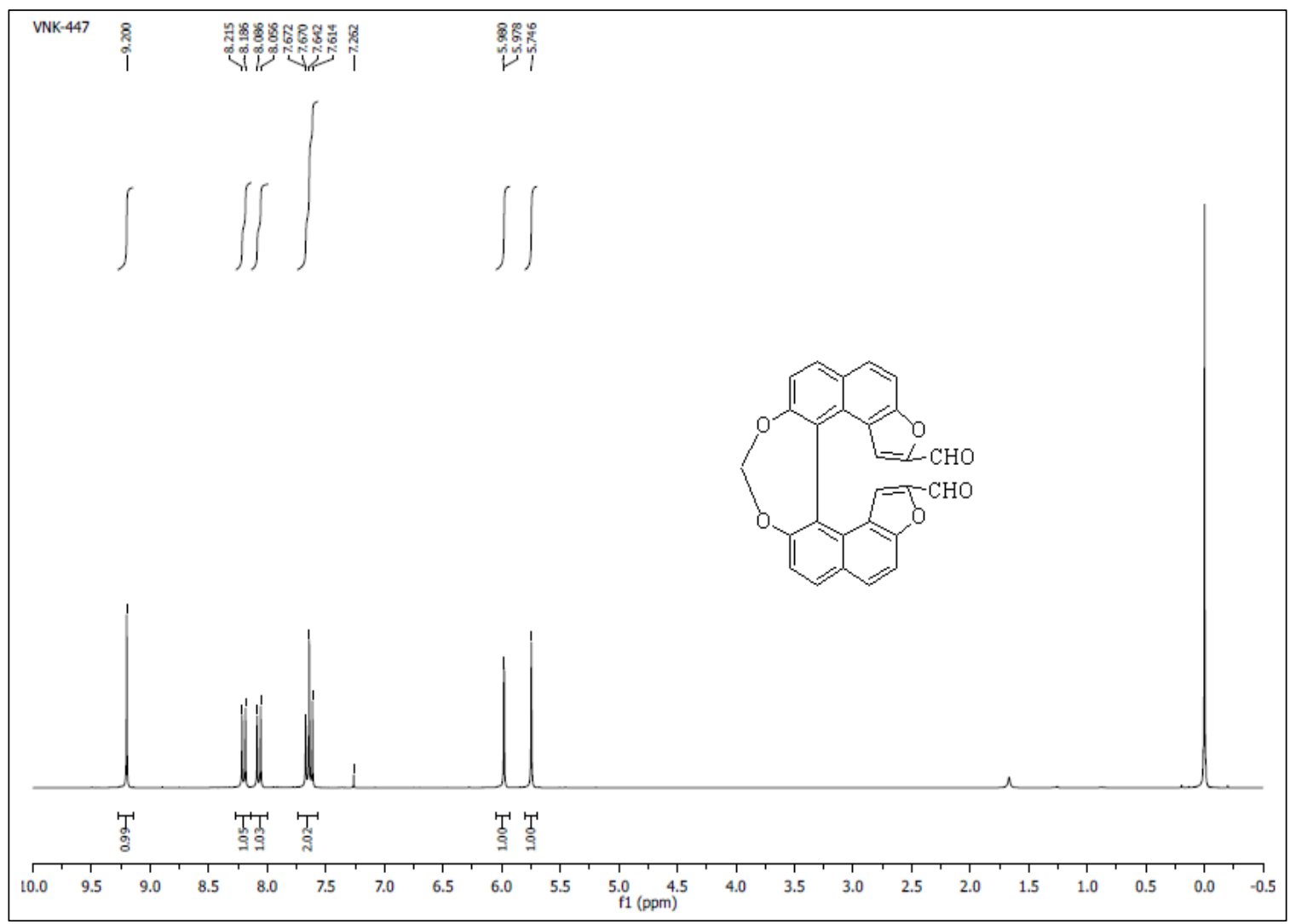

Figure S26. ${ }^{1} \mathrm{H}$ NMR (300 MHz, $\mathrm{CDCl}_{3}$, ppm) of helicenoid di aldehyde, 7. 


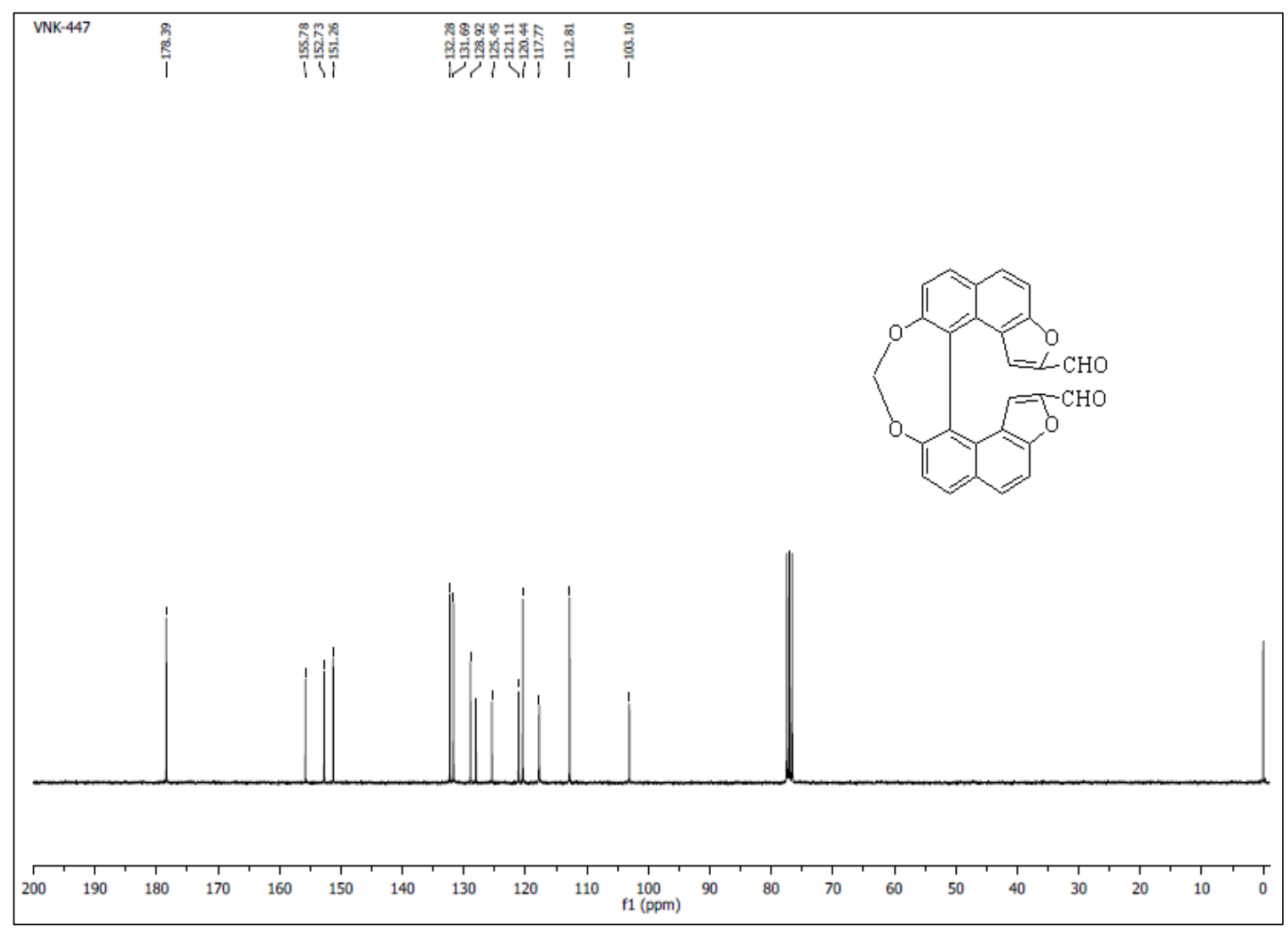

Figure S27. ${ }^{13} \mathrm{C}\left\{{ }^{1} \mathrm{H}\right\}$ NMR $\left(75 \mathrm{MHz}, \mathrm{CDCl}_{3}, \mathrm{ppm}\right)$ of helicenoid di aldehyde, 7.

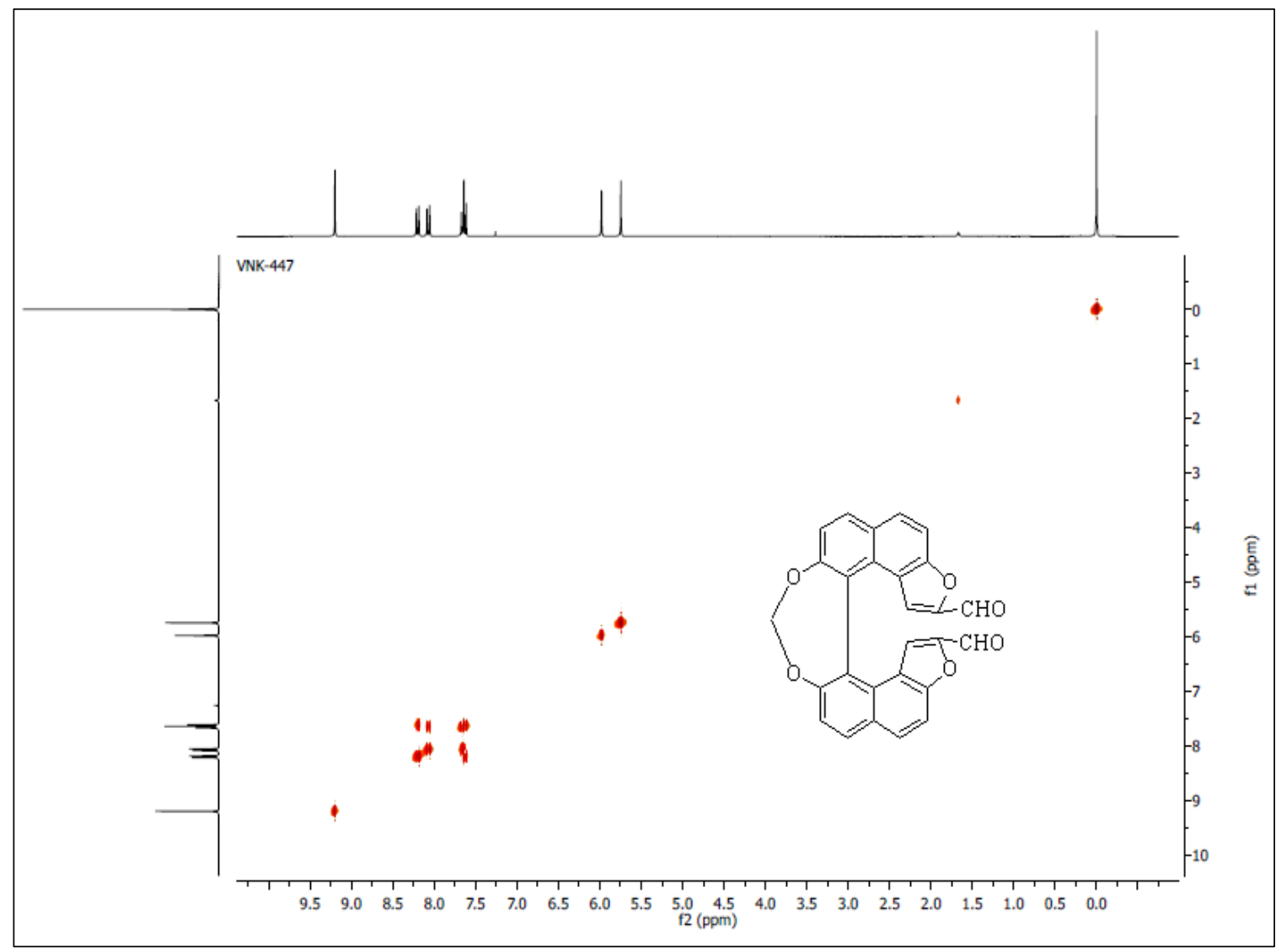

Figure S28. ${ }^{1} \mathrm{H}-{ }^{1} \mathrm{H}$ COSY $\left(300 \mathrm{MHz}, \mathrm{CDCl}_{3}, \mathrm{ppm}\right)$ of helicenoid di aldehyde, 7. 


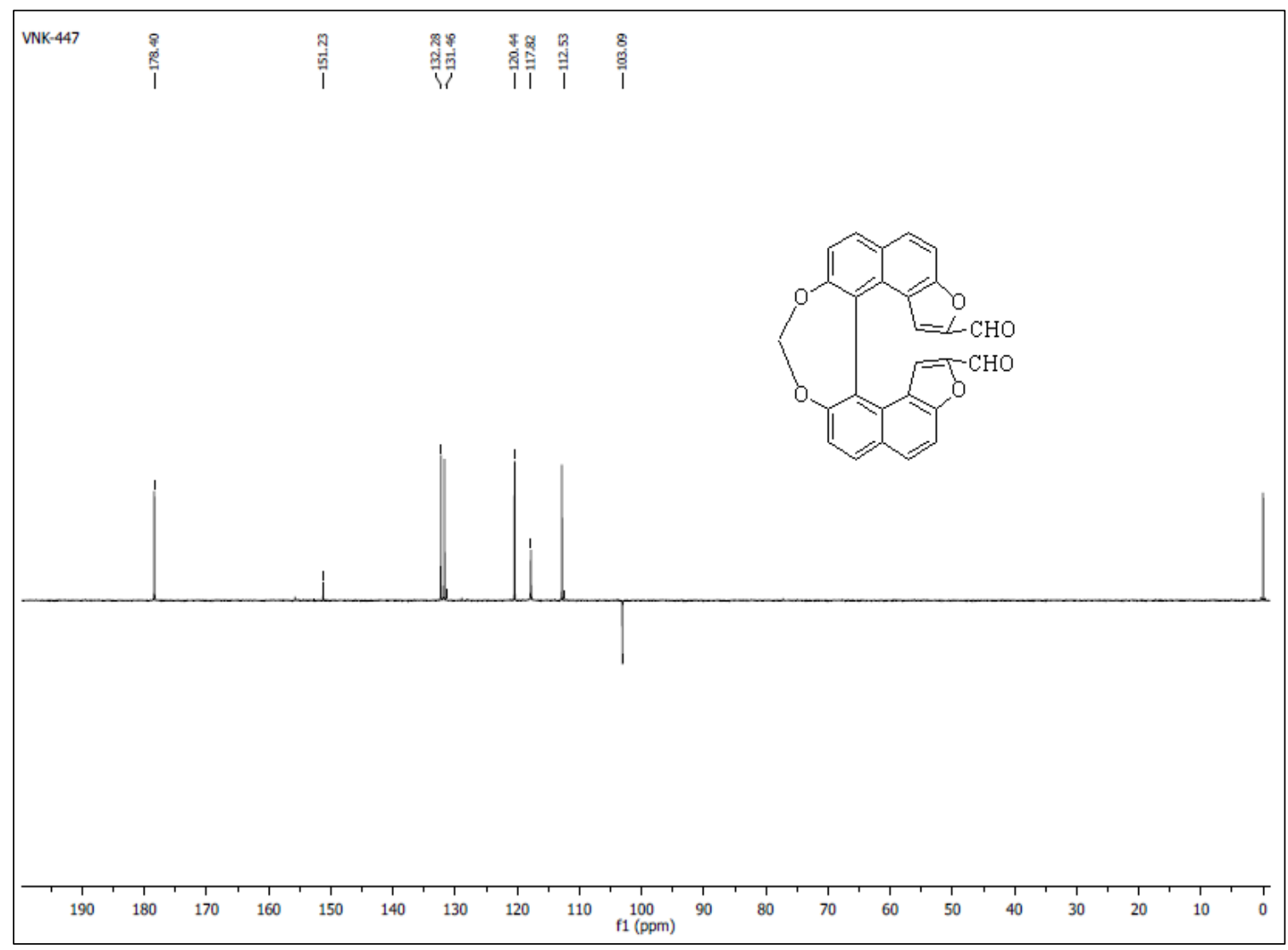

Figure S29. DEPT-135 NMR (75 MHz, $\left.\mathrm{CDCl}_{3}, \mathrm{ppm}\right)$ of helicenoid di aldehyde, 7.

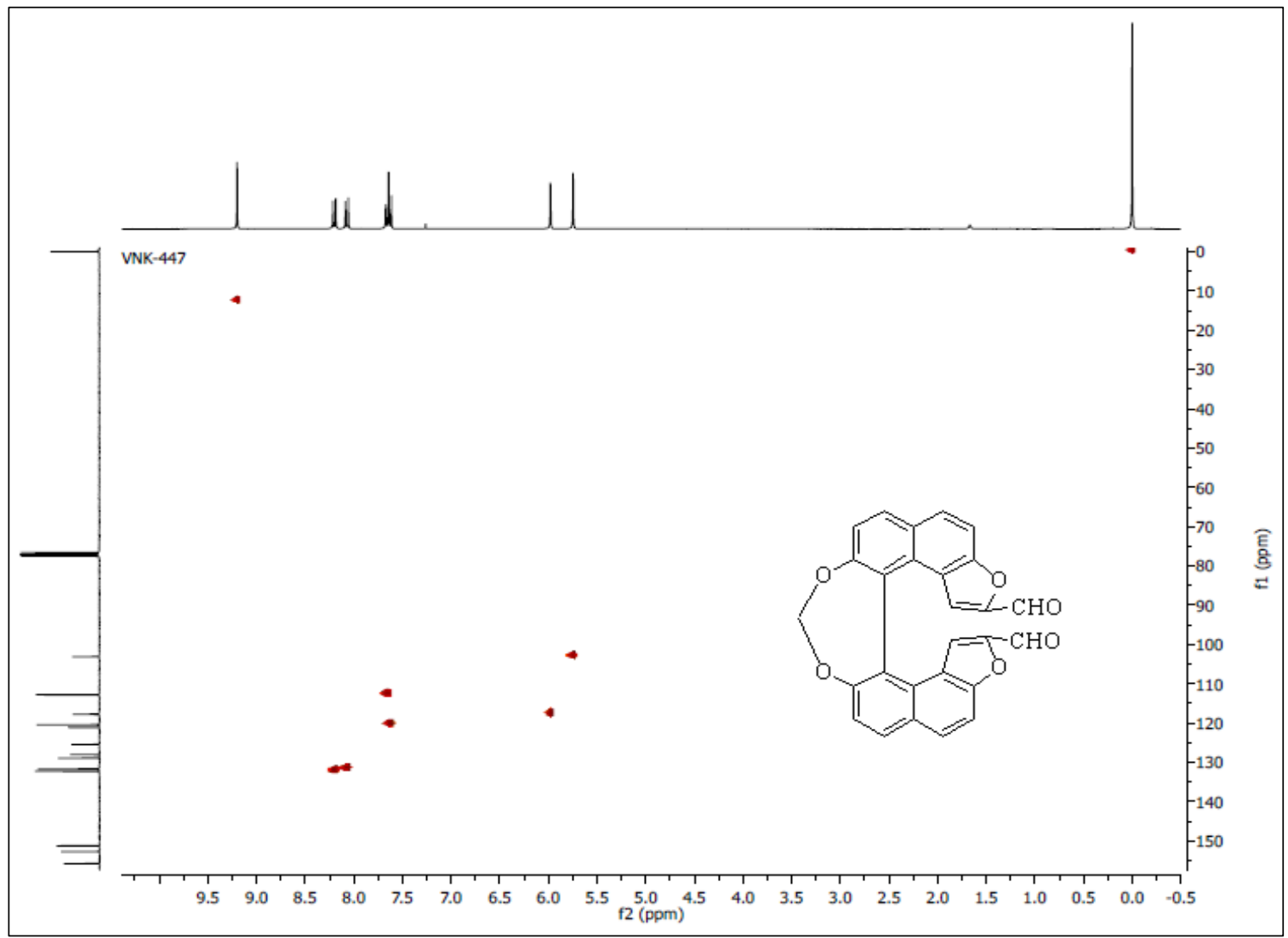

Figure S30. ${ }^{1} \mathrm{H}-{ }^{13} \mathrm{C} \mathrm{HSQC}\left(300\right.$ and $75 \mathrm{MHz}, \mathrm{CDCl}_{3}, \mathrm{ppm}$ ) of helicenoid di aldehyde, 7. 


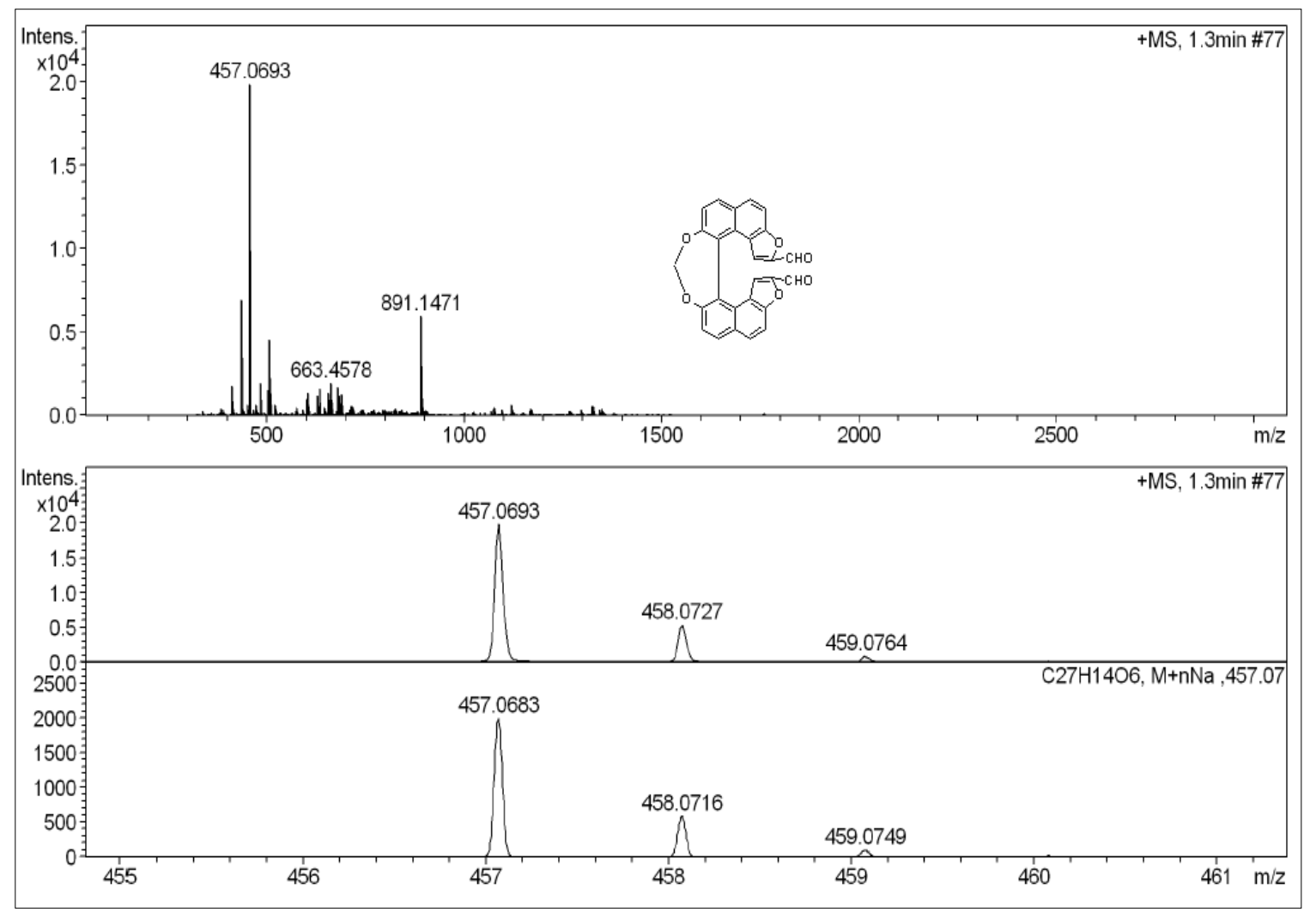

Figure S31. HRMS of helicenoid di aldehyde, 7.

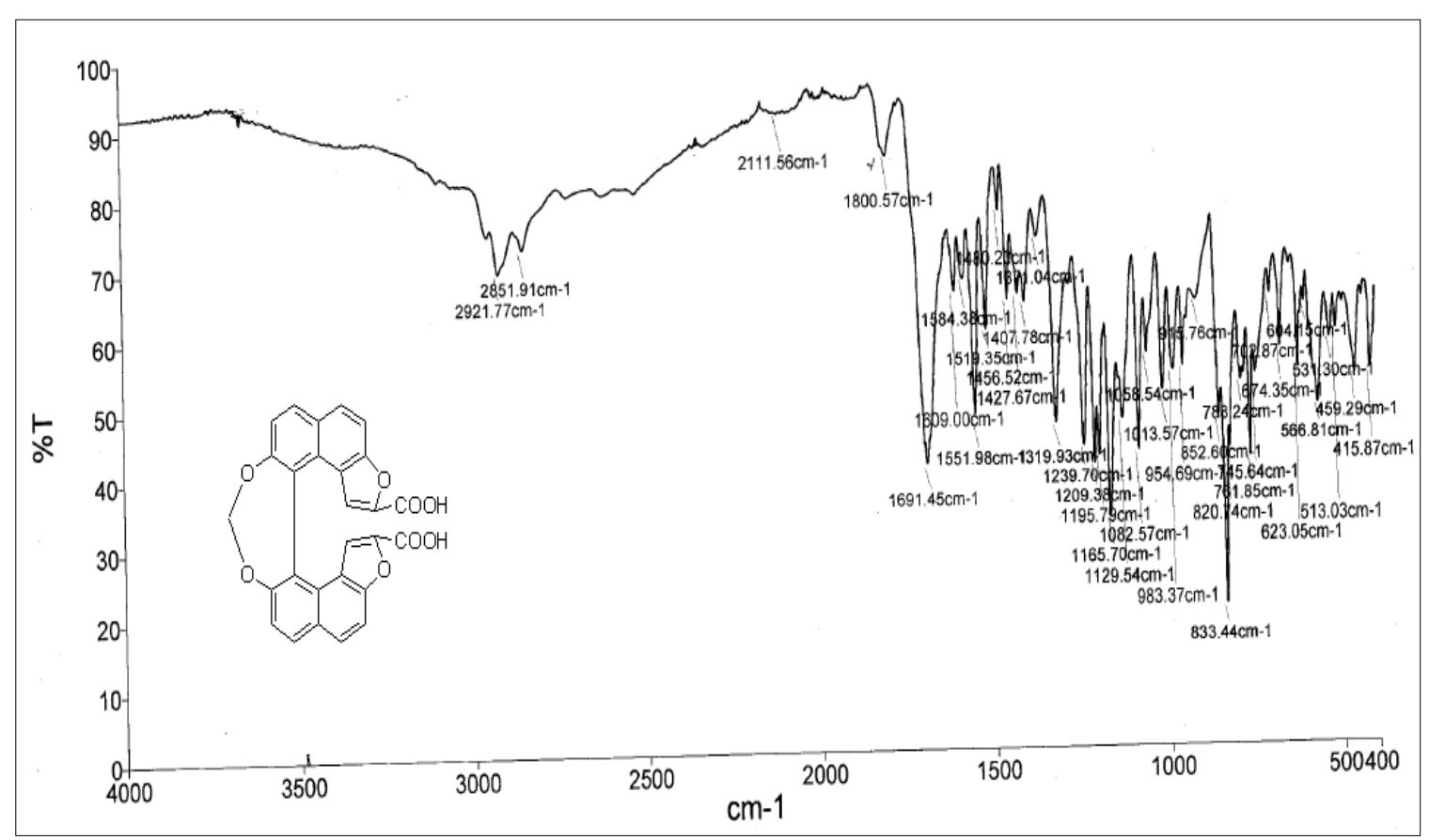

Figure S32. IR of helicenoid dicarboxylic acid, 8. 


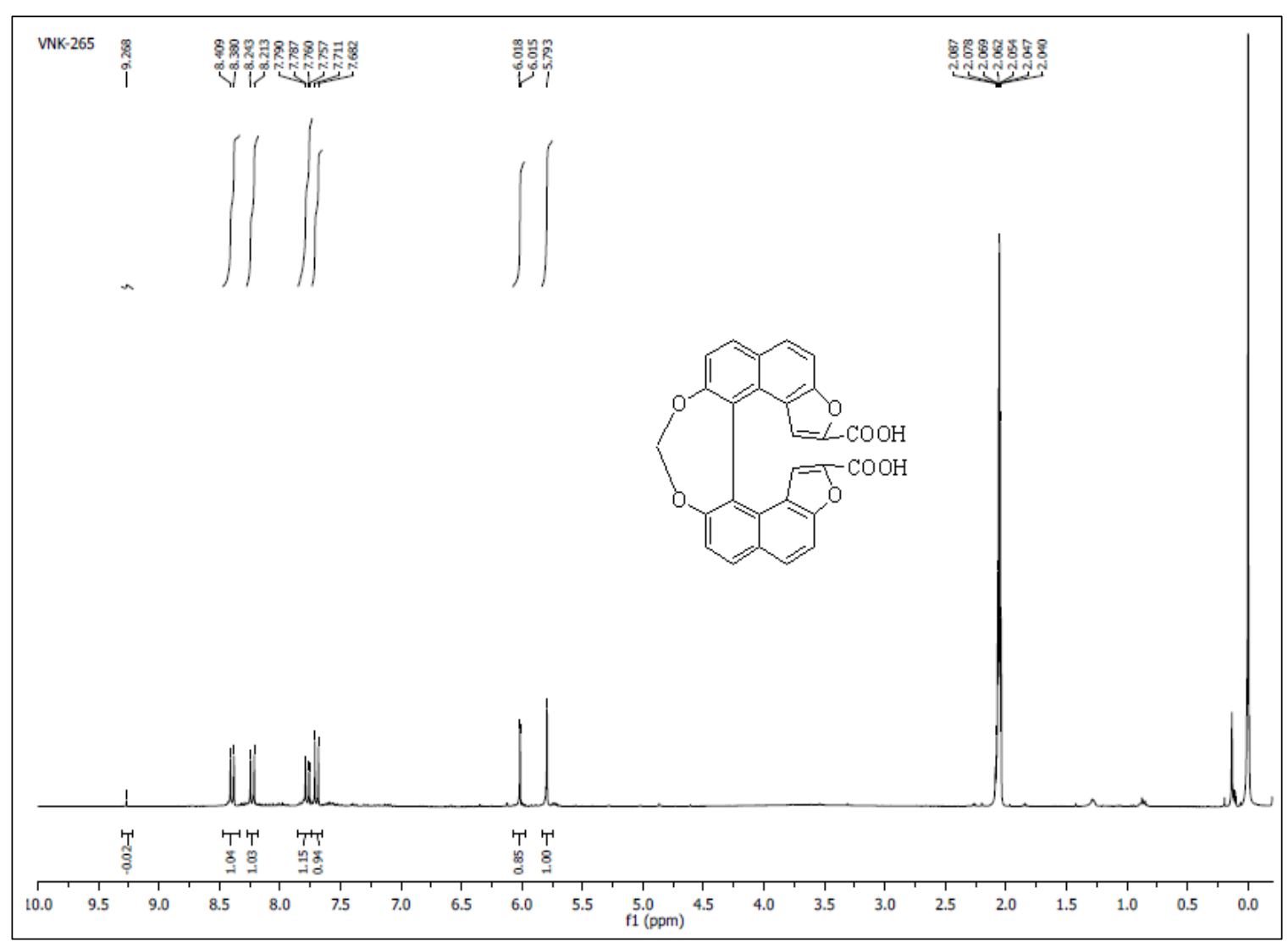

Figure S33. ${ }^{1} \mathrm{H}$ NMR $\left(300 \mathrm{MHz},\left(\mathrm{CD}_{3}\right)_{2} \mathrm{CO}, \mathrm{ppm}\right)$ of helicenoid dicarboxylic acid, 8.

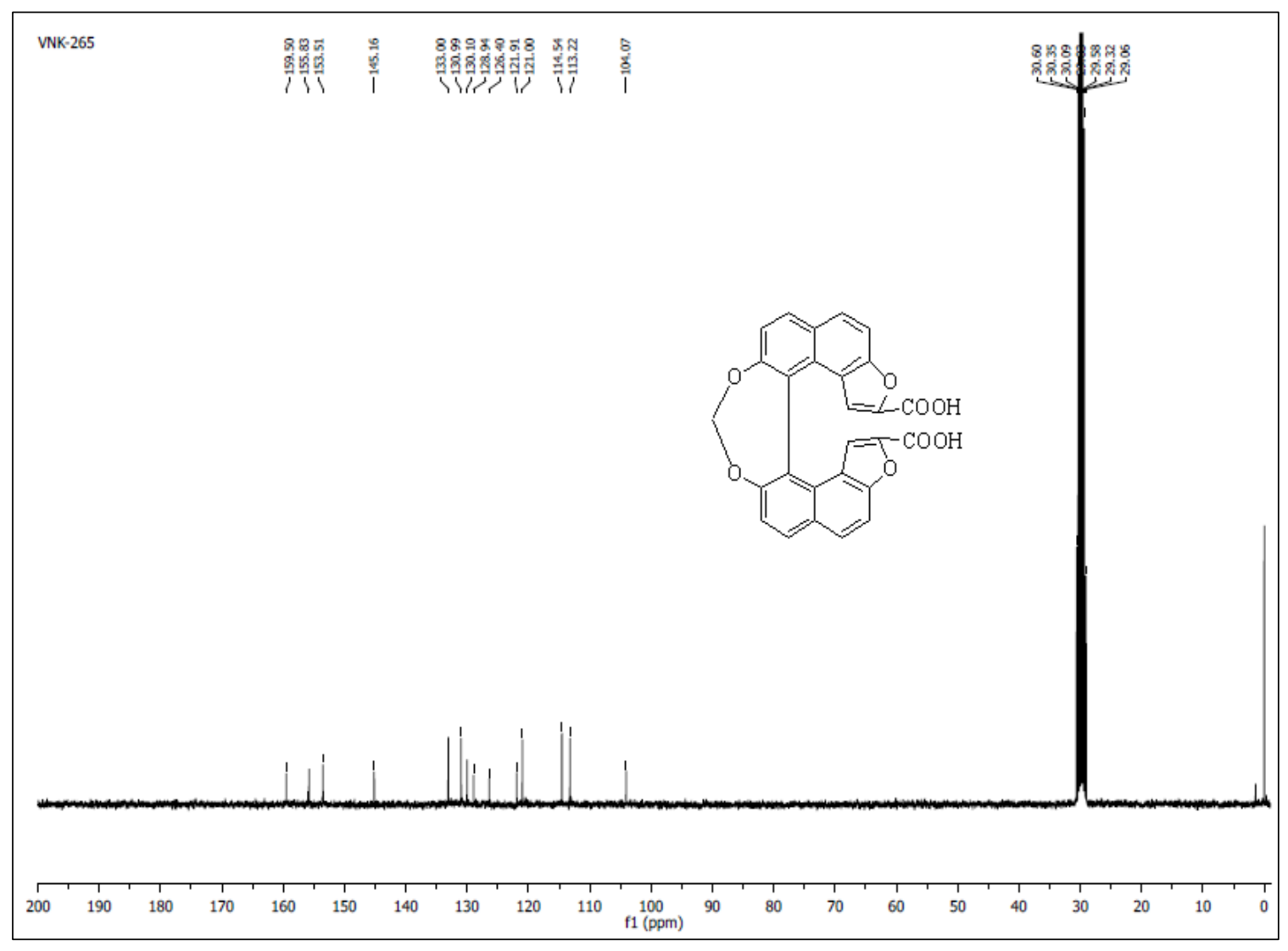

Figure S34. ${ }^{13} \mathrm{C}\left\{{ }^{1} \mathrm{H}\right\}$ NMR $\left(75 \mathrm{MHz},\left(\mathrm{CD}_{3}\right)_{2} \mathrm{CO}\right.$, ppm) of helicenoid dicarboxylic acid, 8.

S18 


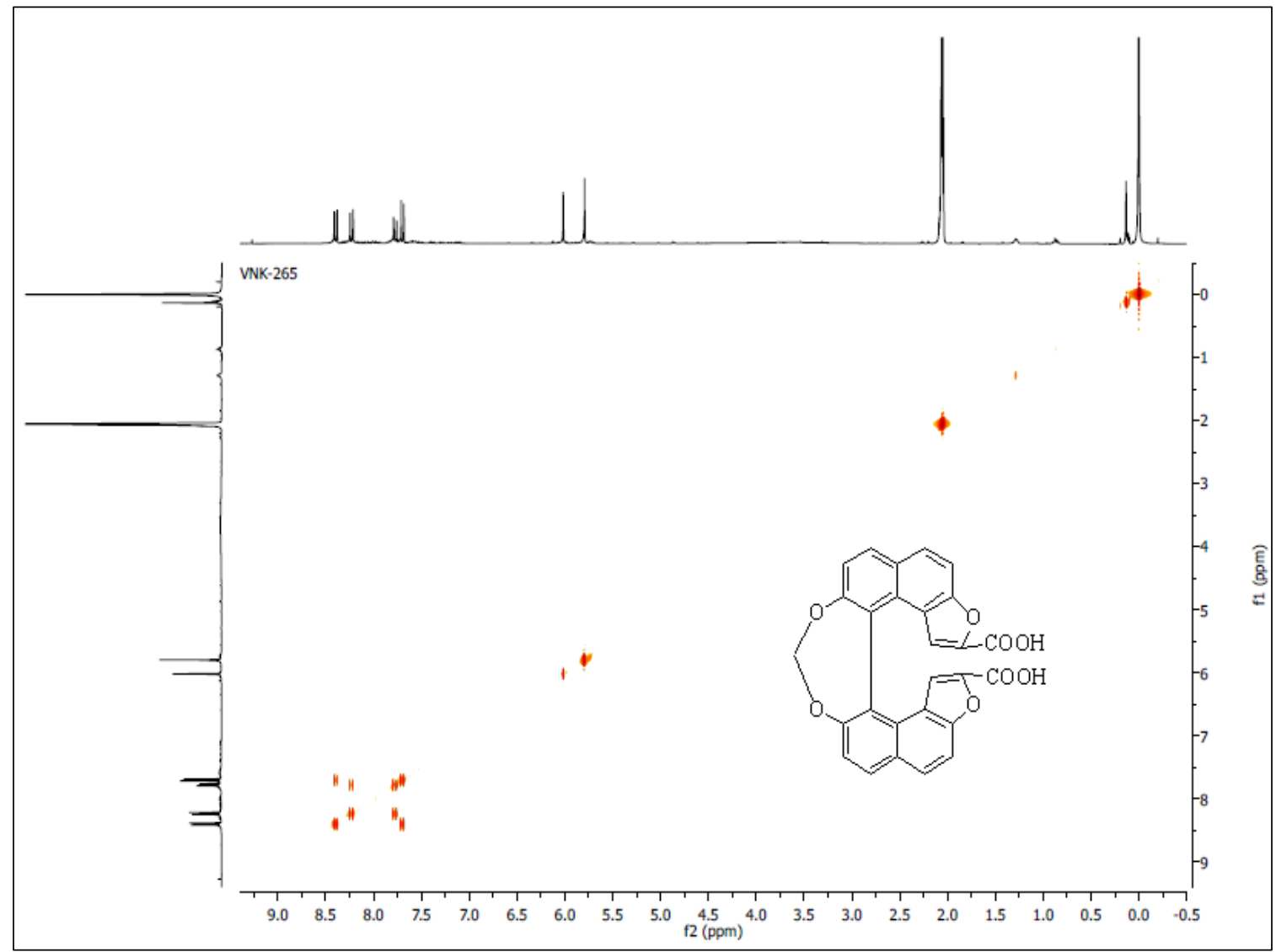

Figure S35. ${ }^{1} \mathrm{H}-{ }^{1} \mathrm{H}$ COSY $\left(300 \mathrm{MHz},\left(\mathrm{CD}_{3}\right)_{2} \mathrm{CO}, \mathrm{ppm}\right)$ of helicenoid dicarboxylic acid, 8 .

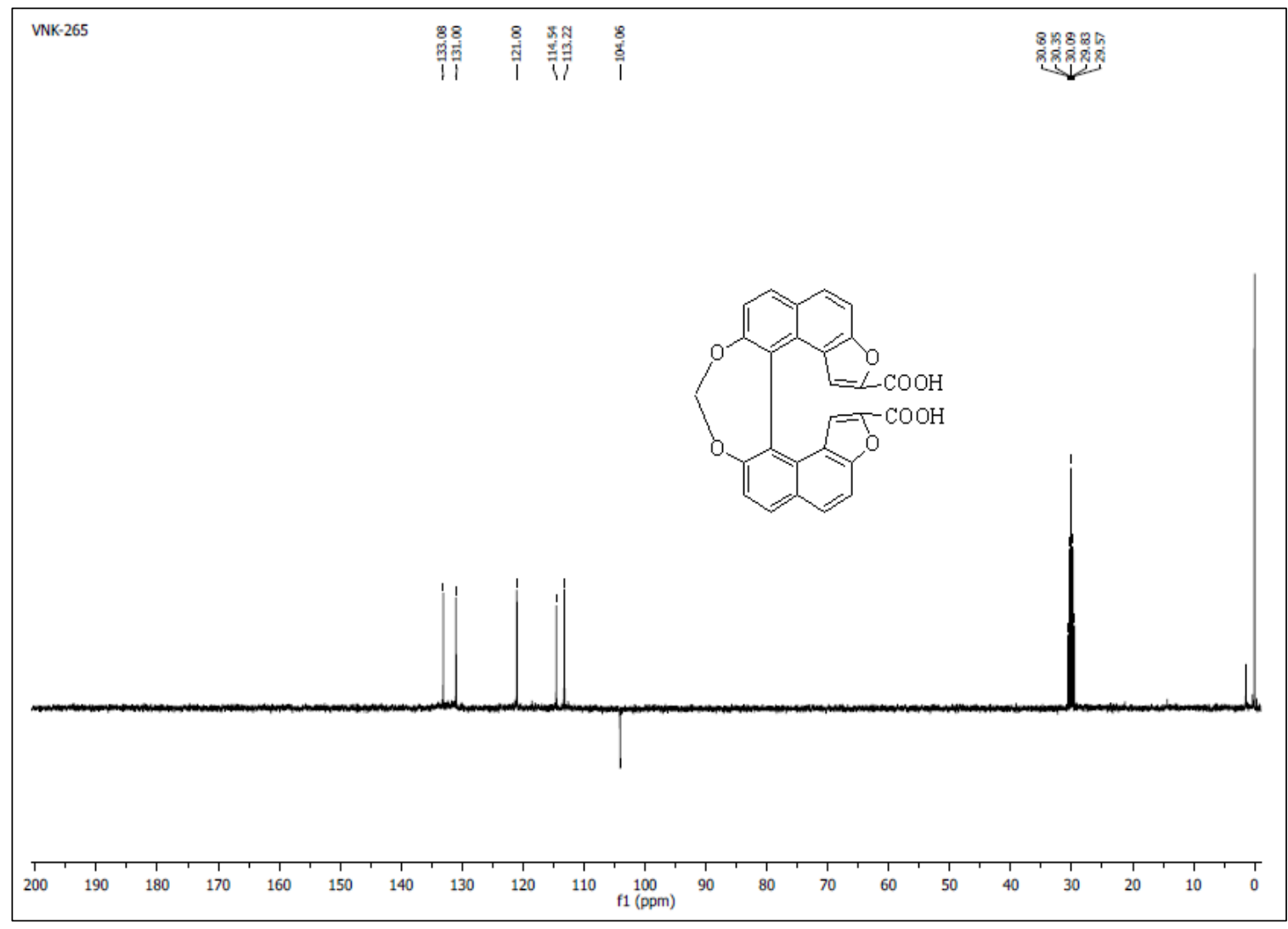

Figure S36: DEPT-135 NMR (75 MHz, $\left(\mathrm{CD}_{3}\right)_{2} \mathrm{CO}$, ppm) of helicenoid dicarboxylic acid, 8 . 


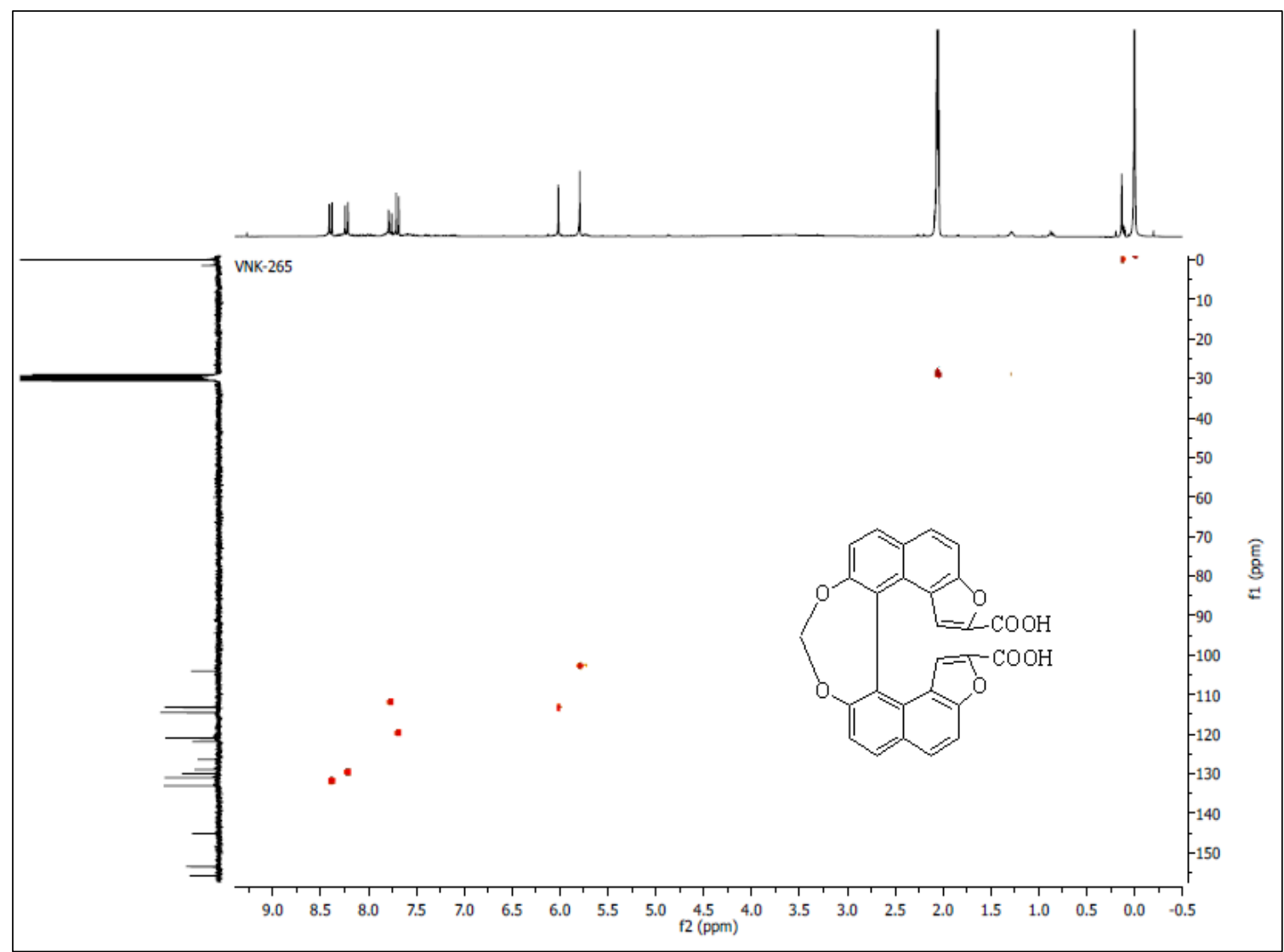

Figure S37: ${ }^{1} \mathrm{H}^{13}{ }^{13} \mathrm{HSQC}\left(300\right.$ and $75 \mathrm{MHz},\left(\mathrm{CD}_{3}\right)_{2} \mathrm{CO}$, ppm) of helicenoid dicarboxylic acid, 8 .

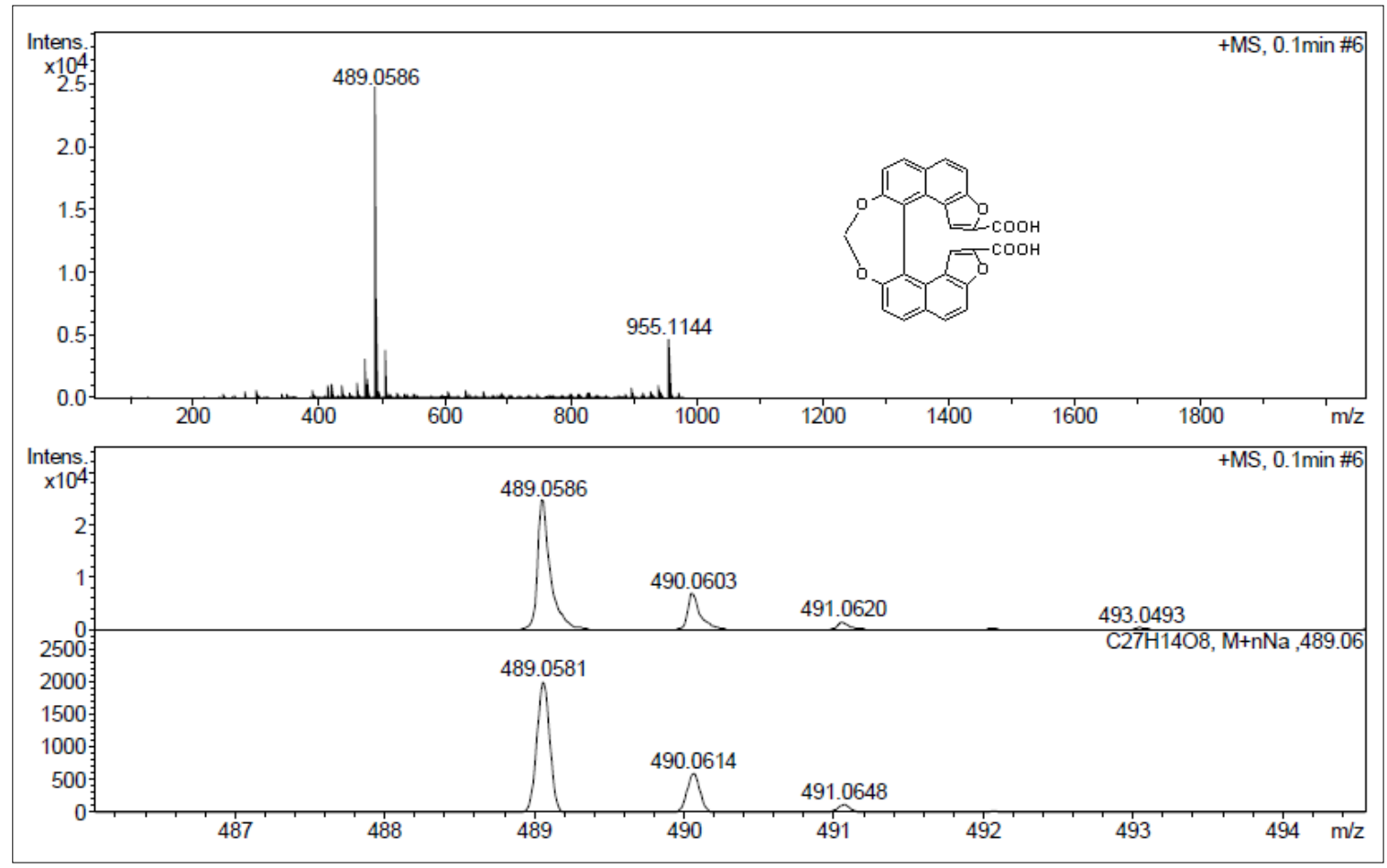

Figure S38. HRMS of helicenoid dicarboxylic acid, 8 . 


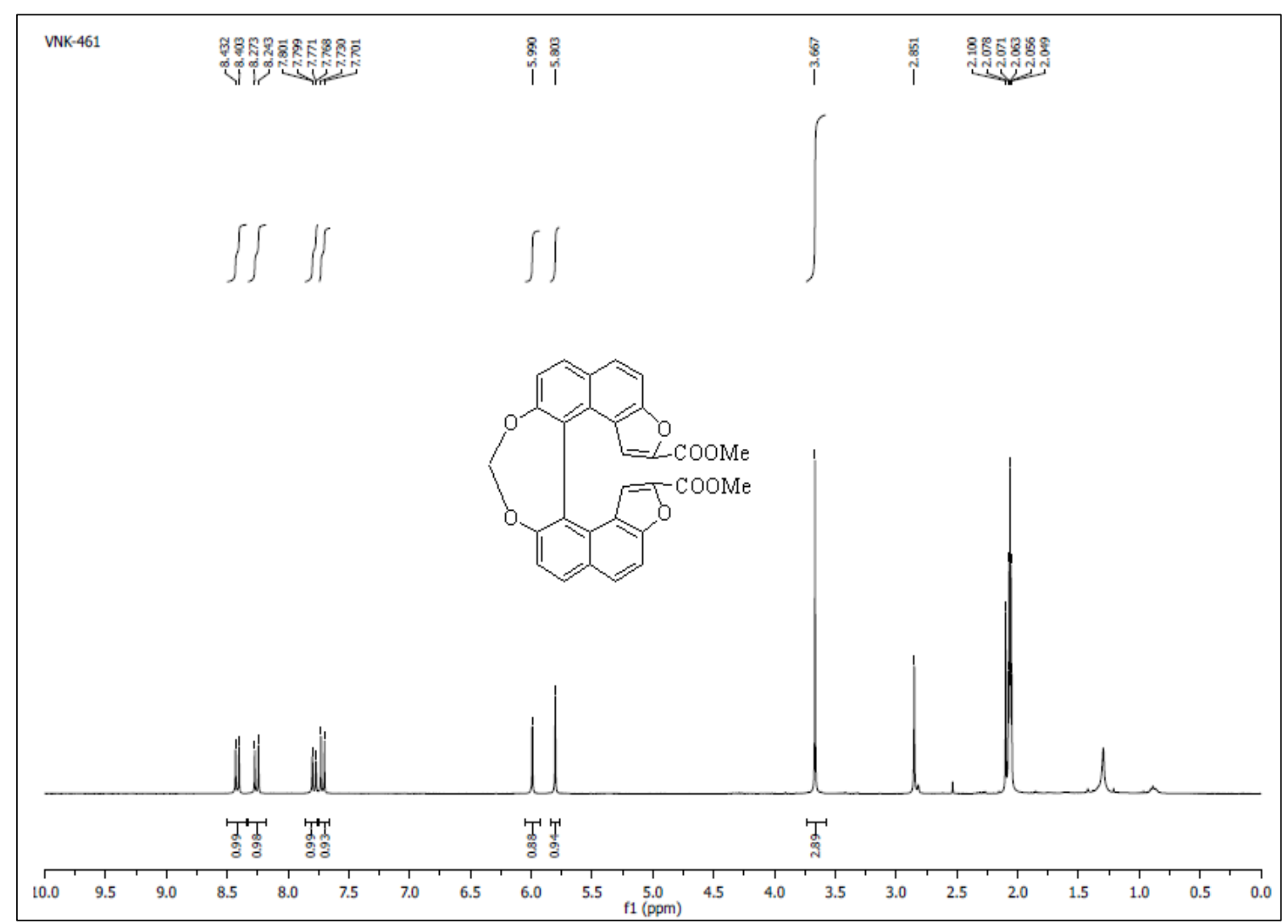

Figure S39. ${ }^{1} \mathrm{H}$ NMR $\left(300 \mathrm{MHz},\left(\mathrm{CD}_{3}\right)_{2} \mathrm{CO}\right.$, ppm) helicenoid methyl diester, 10.

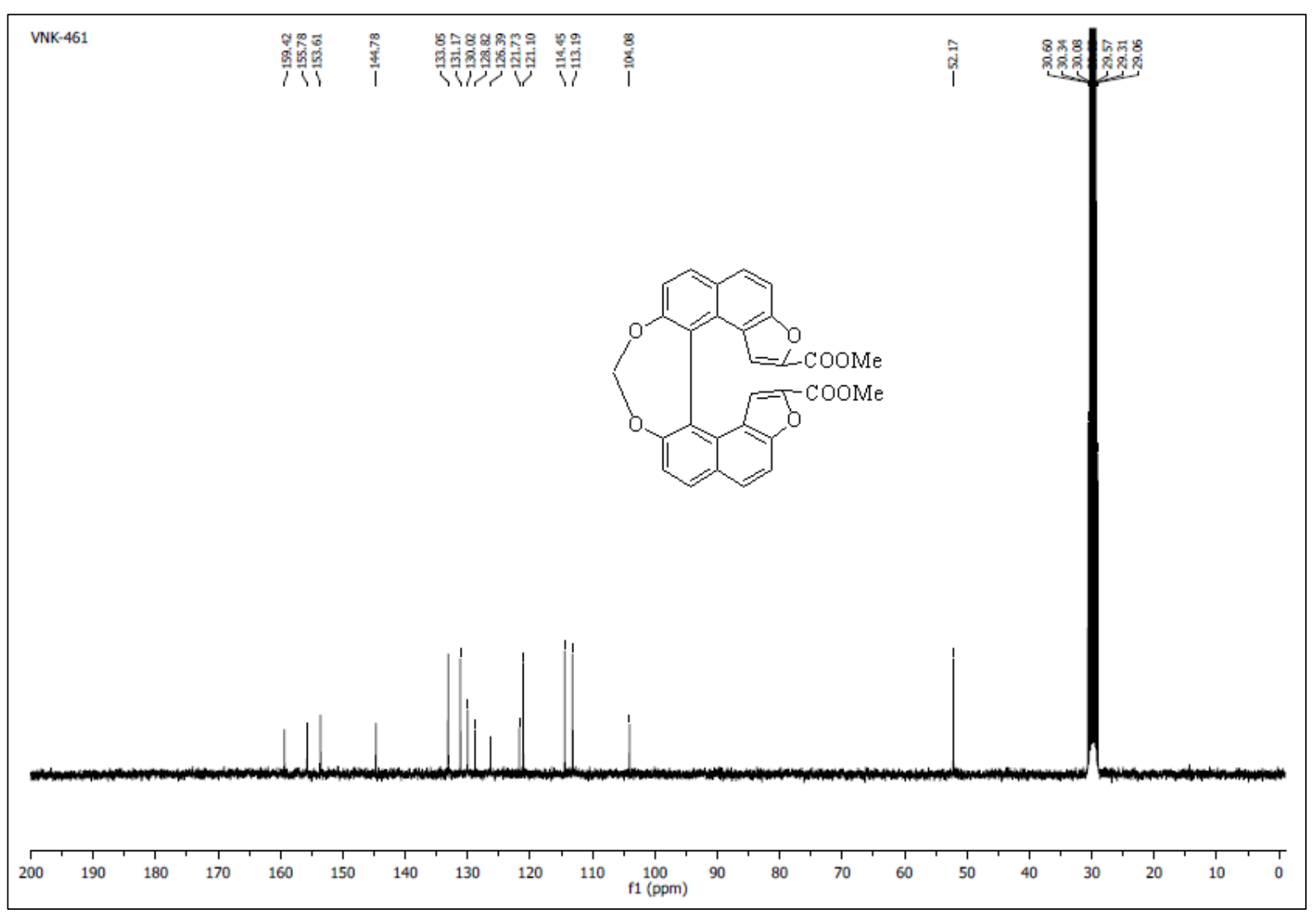

Figure $\mathbf{S 4 0} .{ }^{13} \mathrm{C}\left\{{ }^{1} \mathrm{H}\right\}$ NMR $\left(75 \mathrm{MHz},\left(\mathrm{CD}_{3}\right)_{2} \mathrm{CO}, \mathrm{ppm}\right)$ helicenoid methyl diester, 10. 


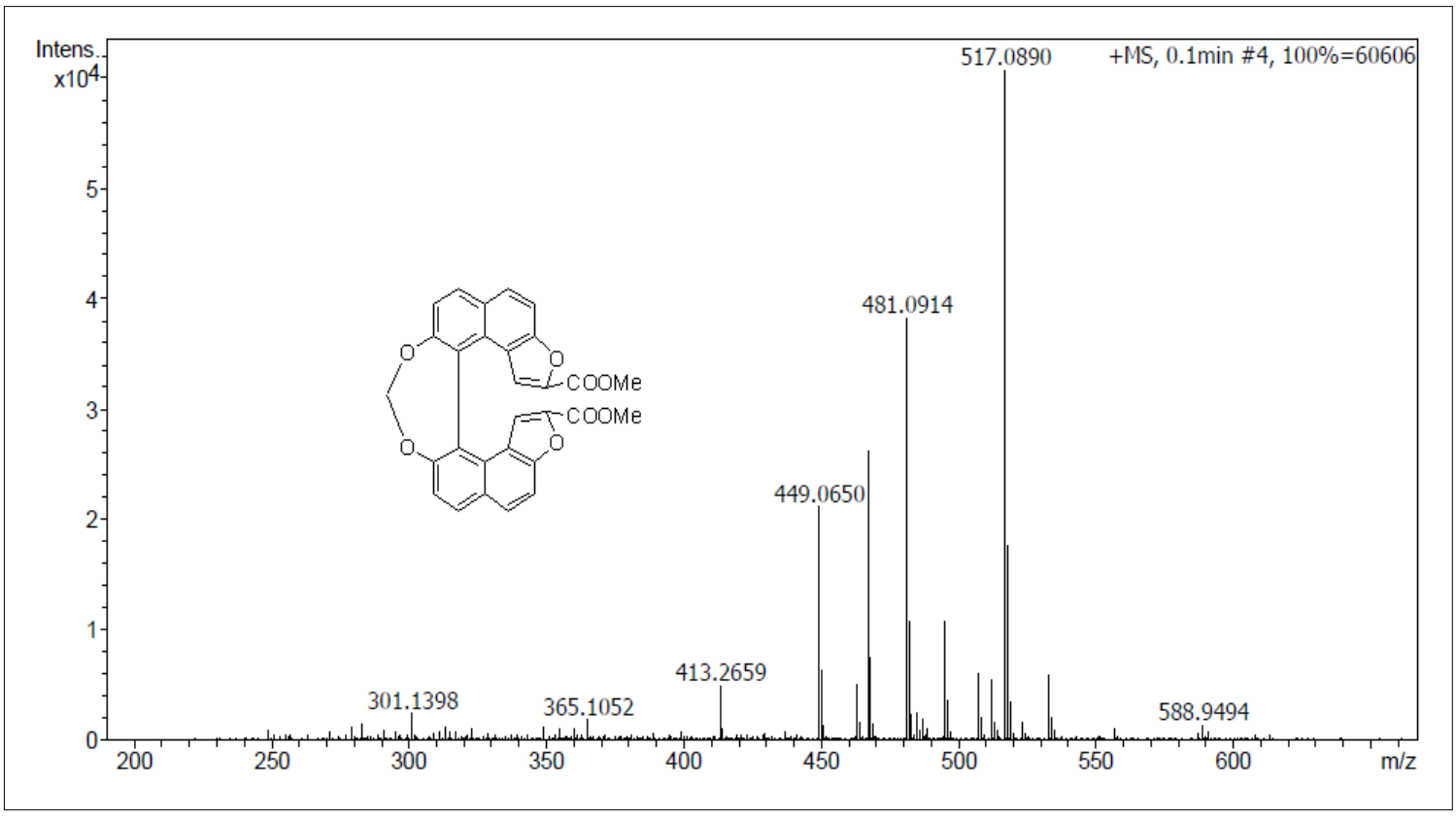

Figure S41. HRMS helicenoid methyl diester, 10.

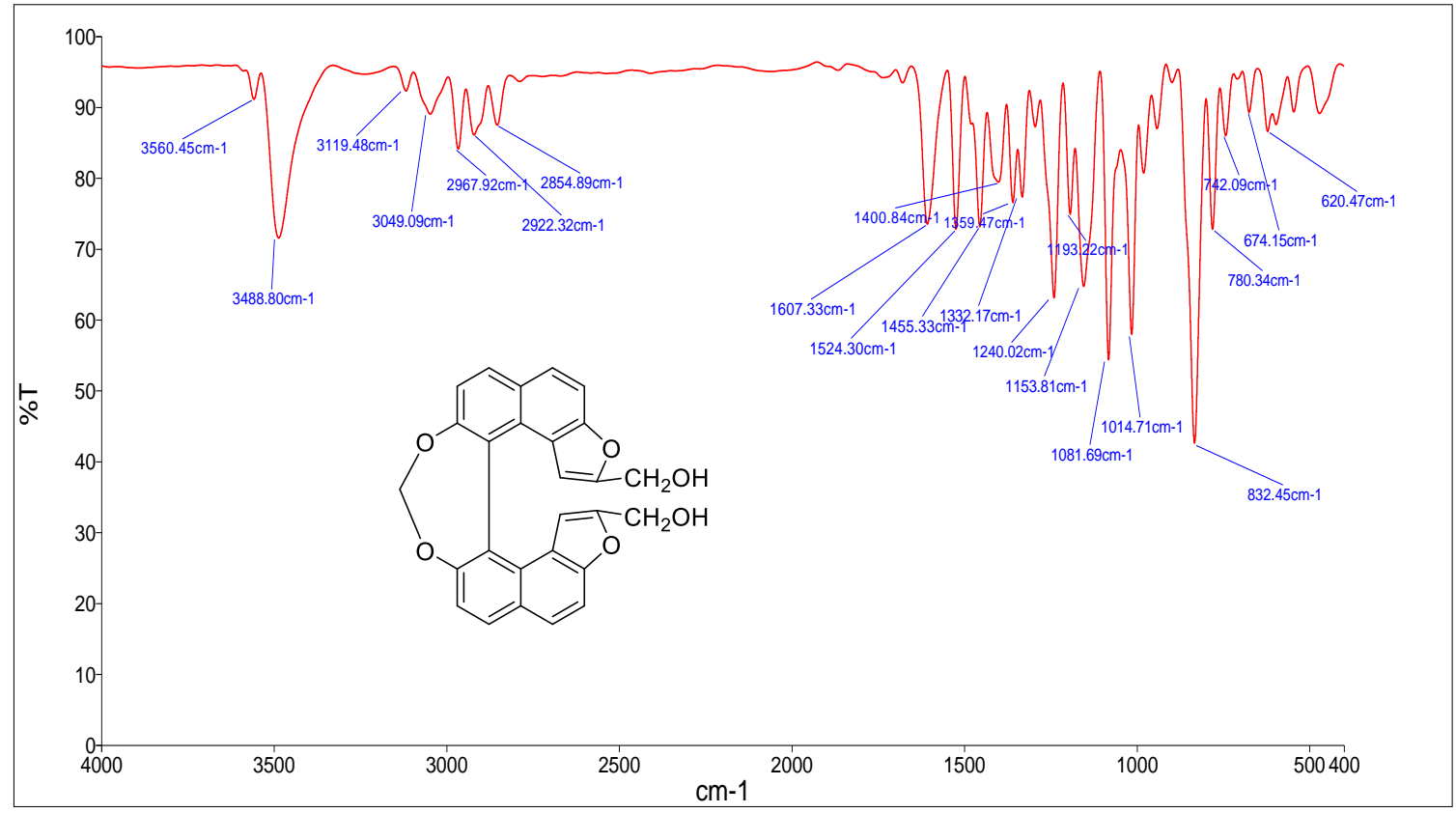

Figure S42. IR of helicenoid diol, 9. 


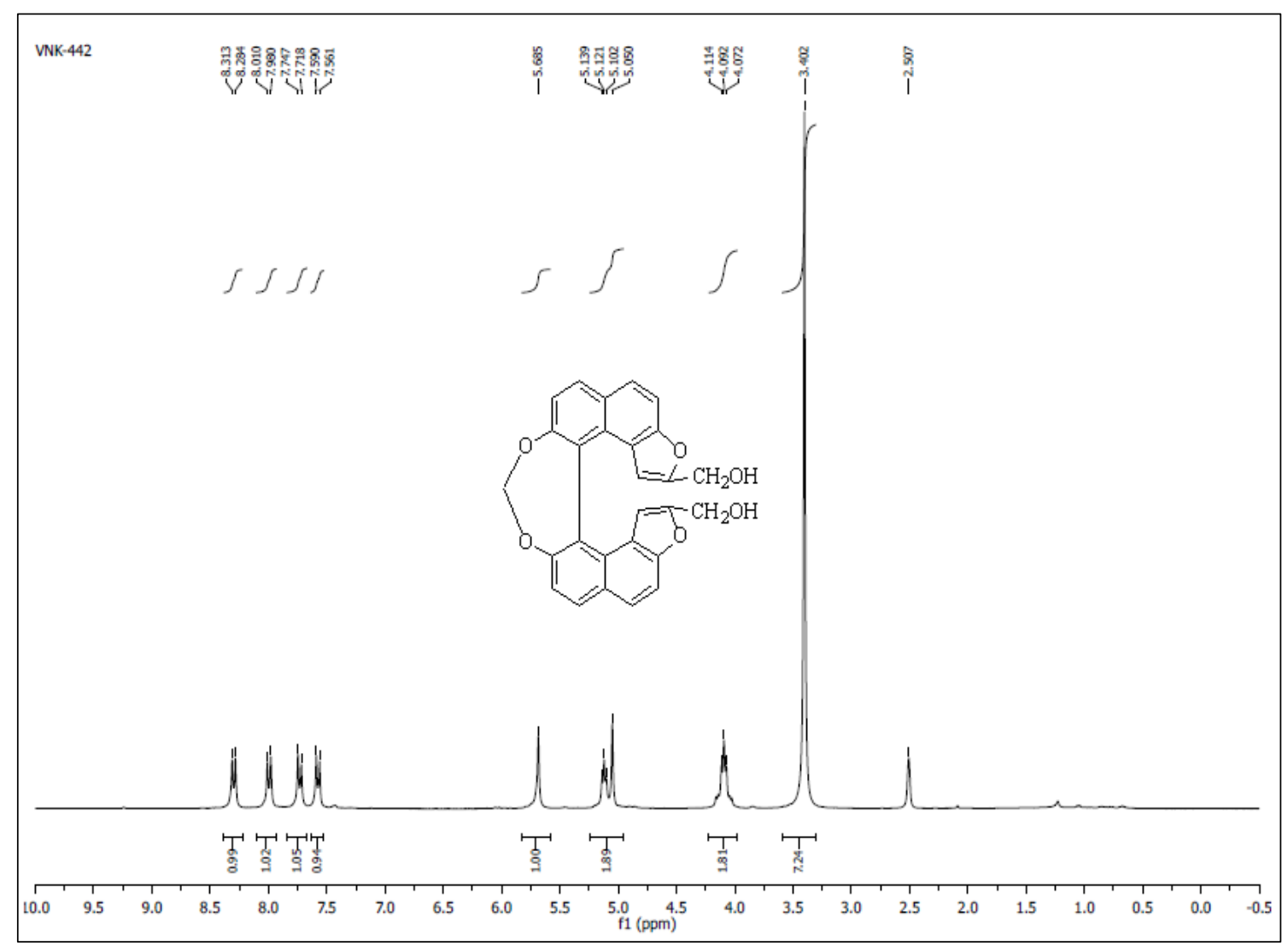

Figure S43. ${ }^{1} \mathrm{H}$ NMR (300 MHz, DMSO-d 6 , ppm) of helicenoid diol, 9.

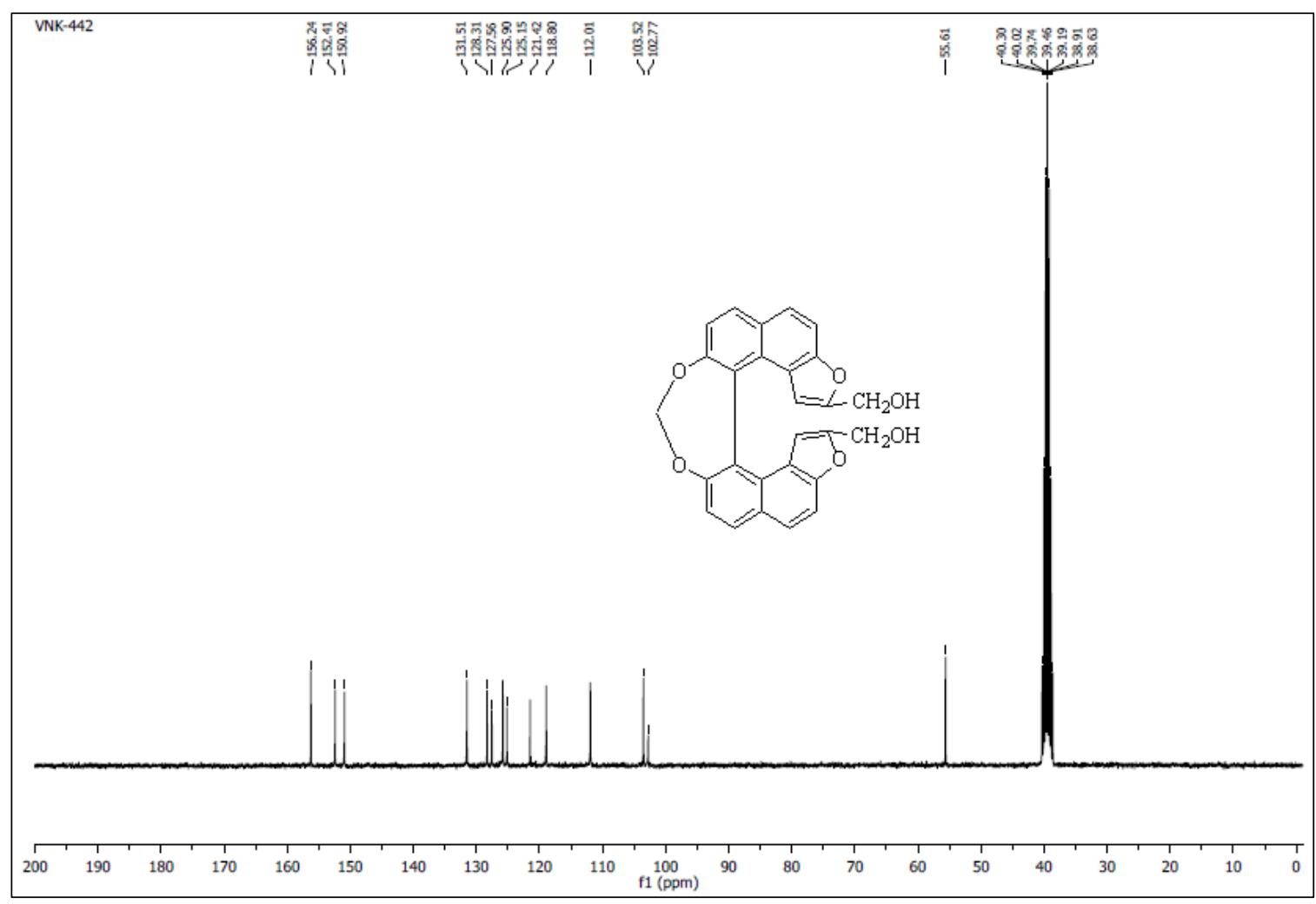

Figure S44. ${ }^{13} \mathrm{C}\left\{{ }^{1} \mathrm{H}\right\}$ NMR (75 MHz, DMSO-d 6 , ppm) of helicenoid diol, 9. 


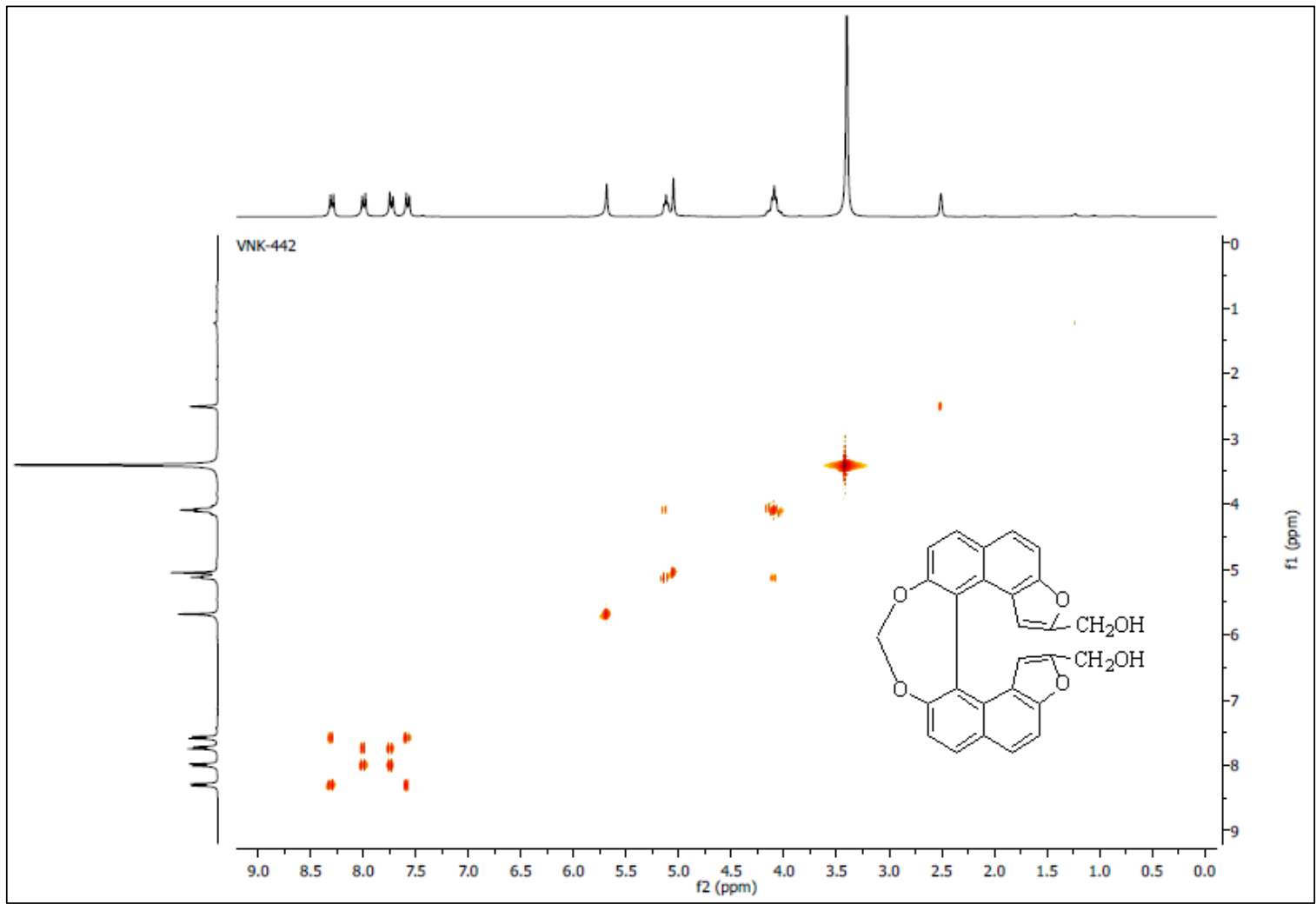

Figure S45. ${ }^{1} \mathrm{H}-{ }^{1} \mathrm{H} \operatorname{COSY}(300 \mathrm{MHz}$, DMSO-d 6 , ppm) of helicenoid diol, 9.

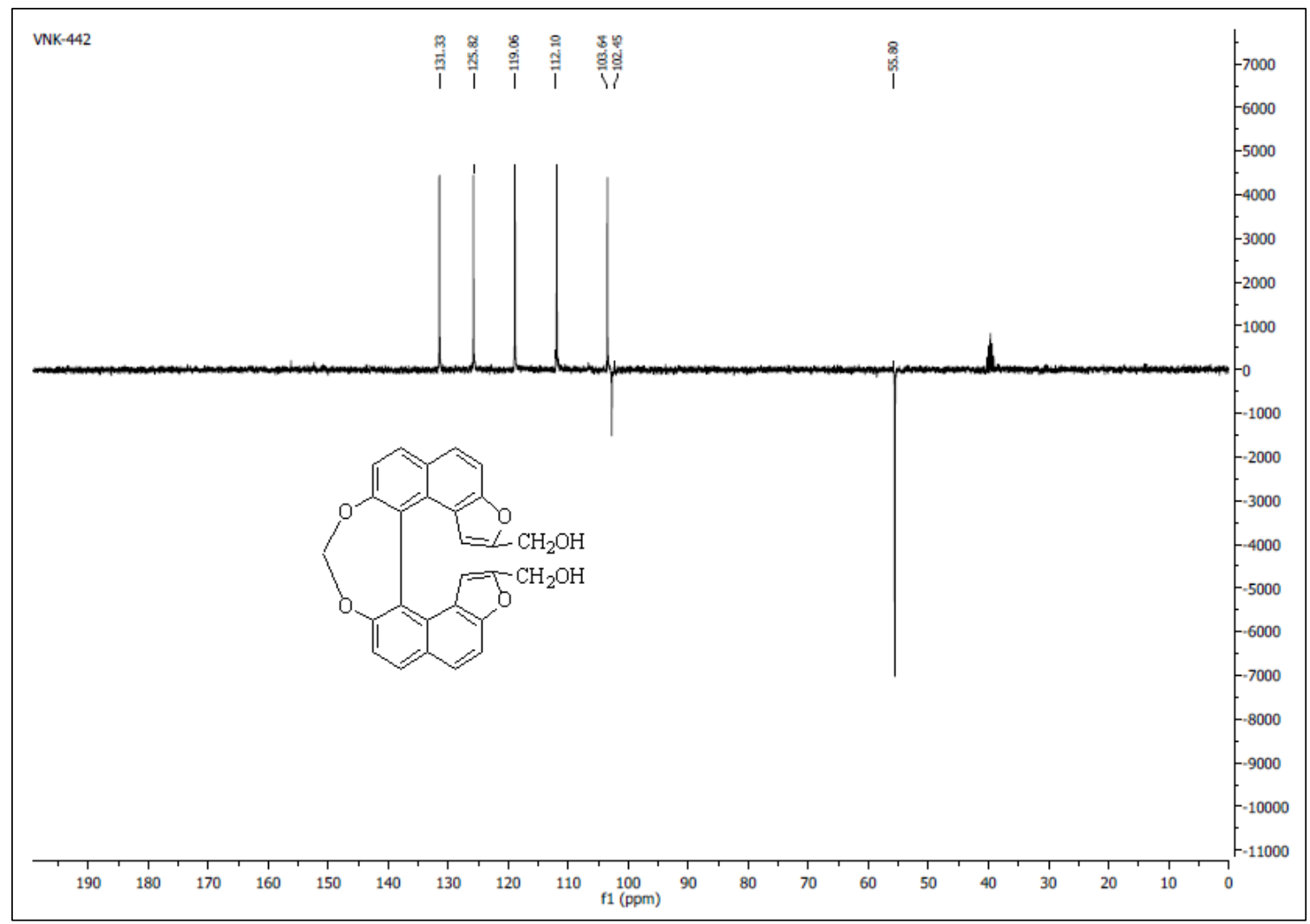

Figure S46. DEPT-135 NMR (75 MHz, DMSO-d 6 , ppm) of helicenoid diol, 9. 


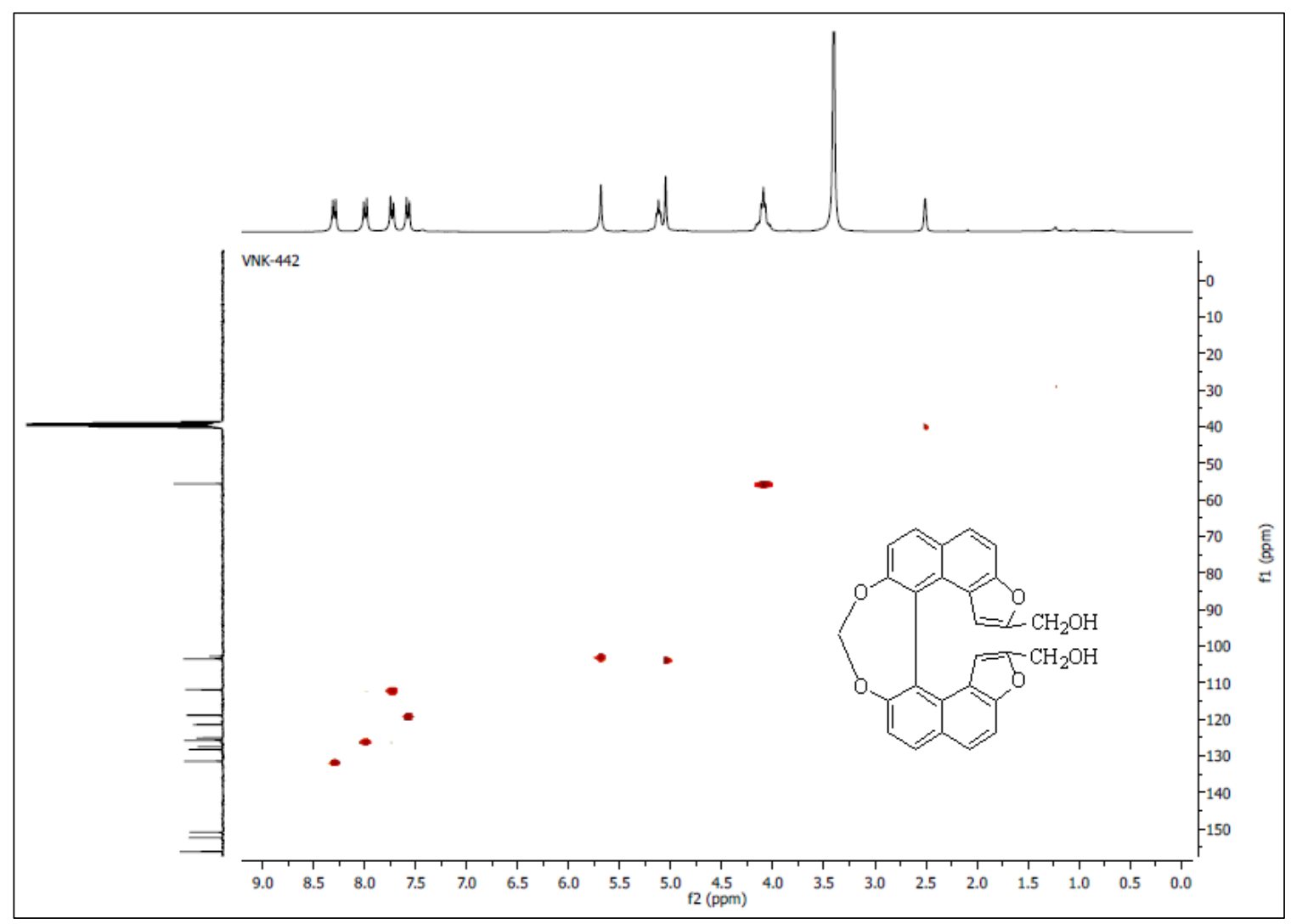

Figure S47. ${ }^{1} \mathrm{H}^{13}{ }^{13} \mathrm{C}$ HSQC (300 and $75 \mathrm{MHz}, \mathrm{DMSO}-\mathrm{d}_{6}, \mathrm{ppm}$ ) of helicenoid diol, 9.

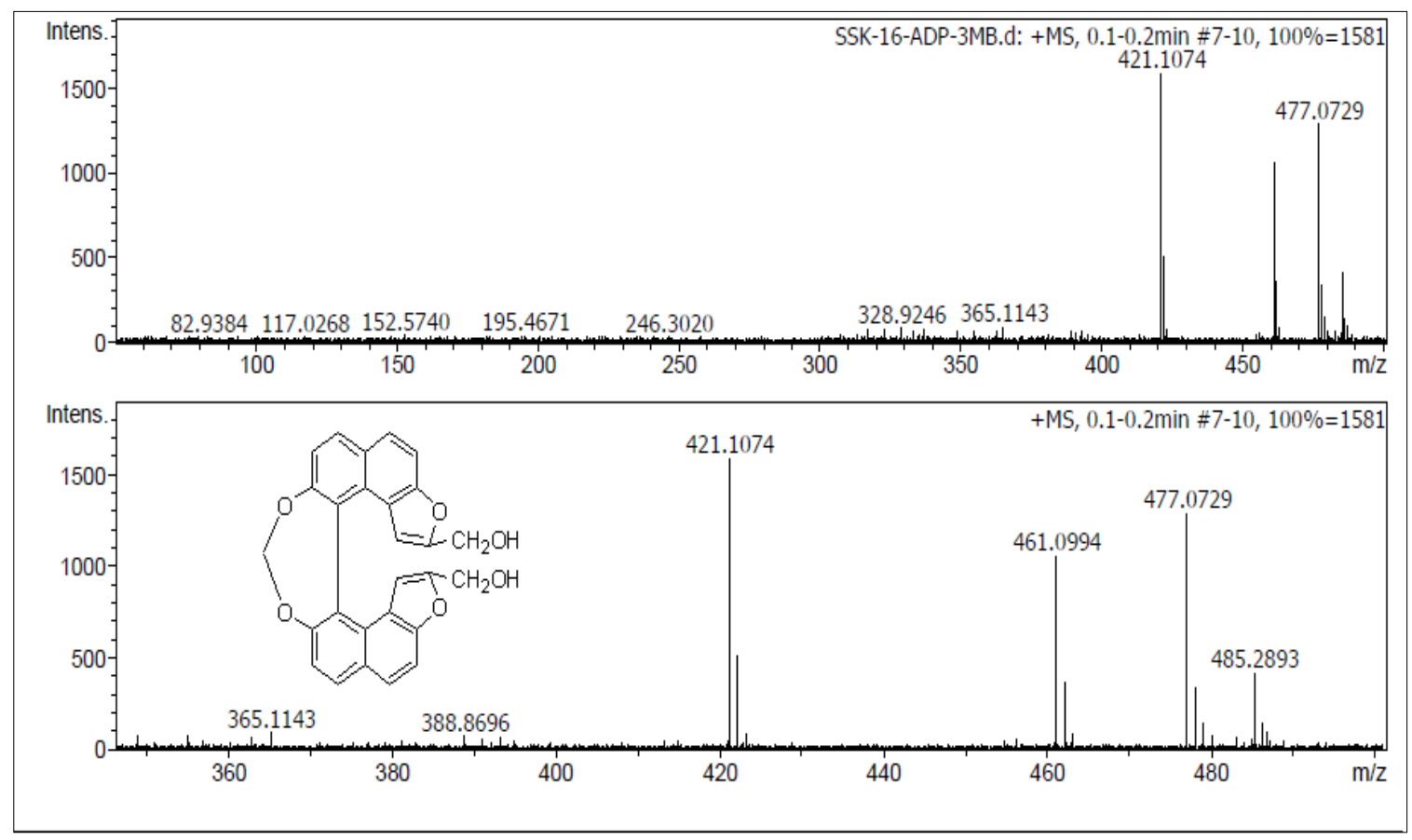

Figure S48. HRMS of helicenoid diol, 9. 


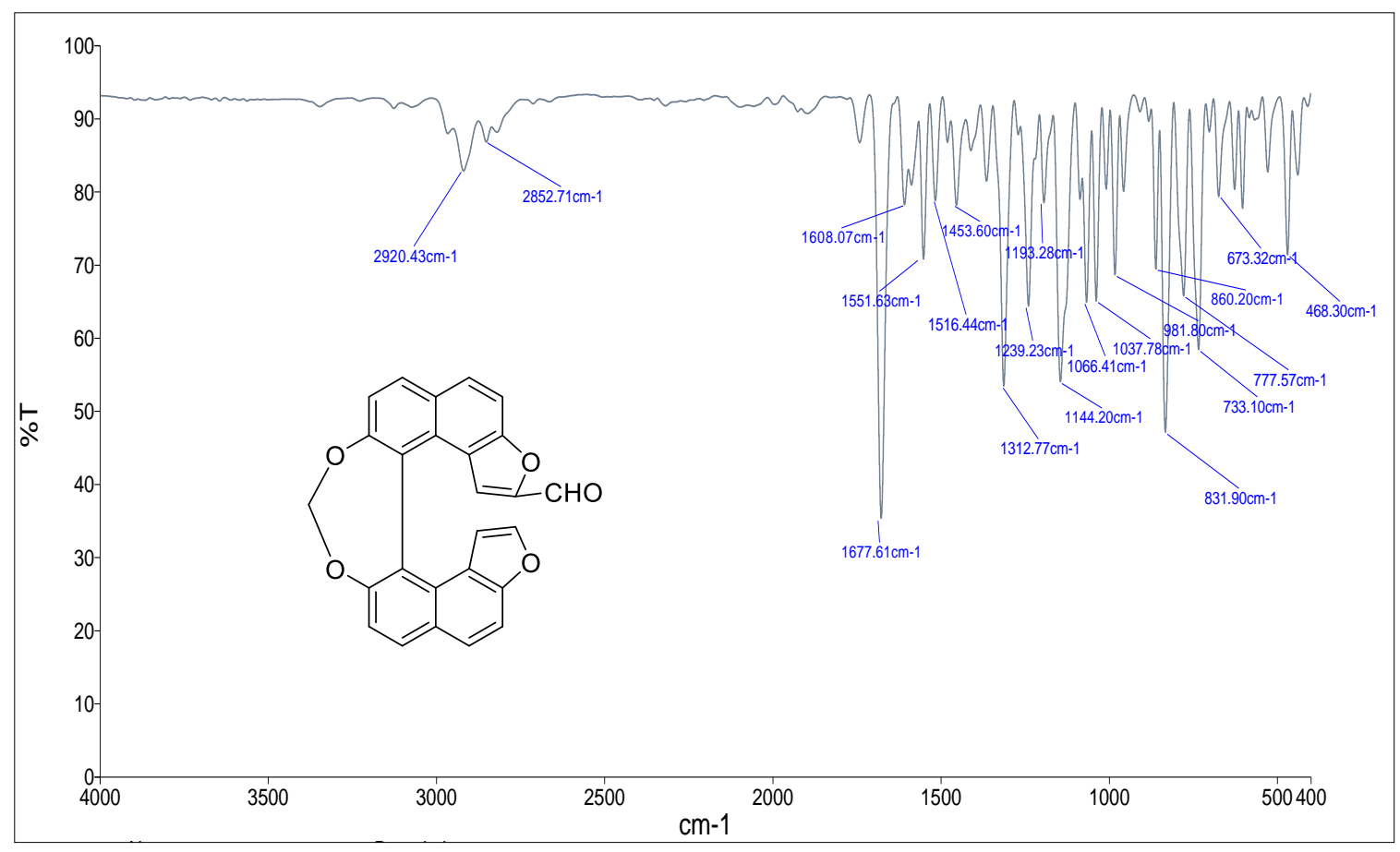

Figure S49. IR of helicenoid mono aldehyde, 11.

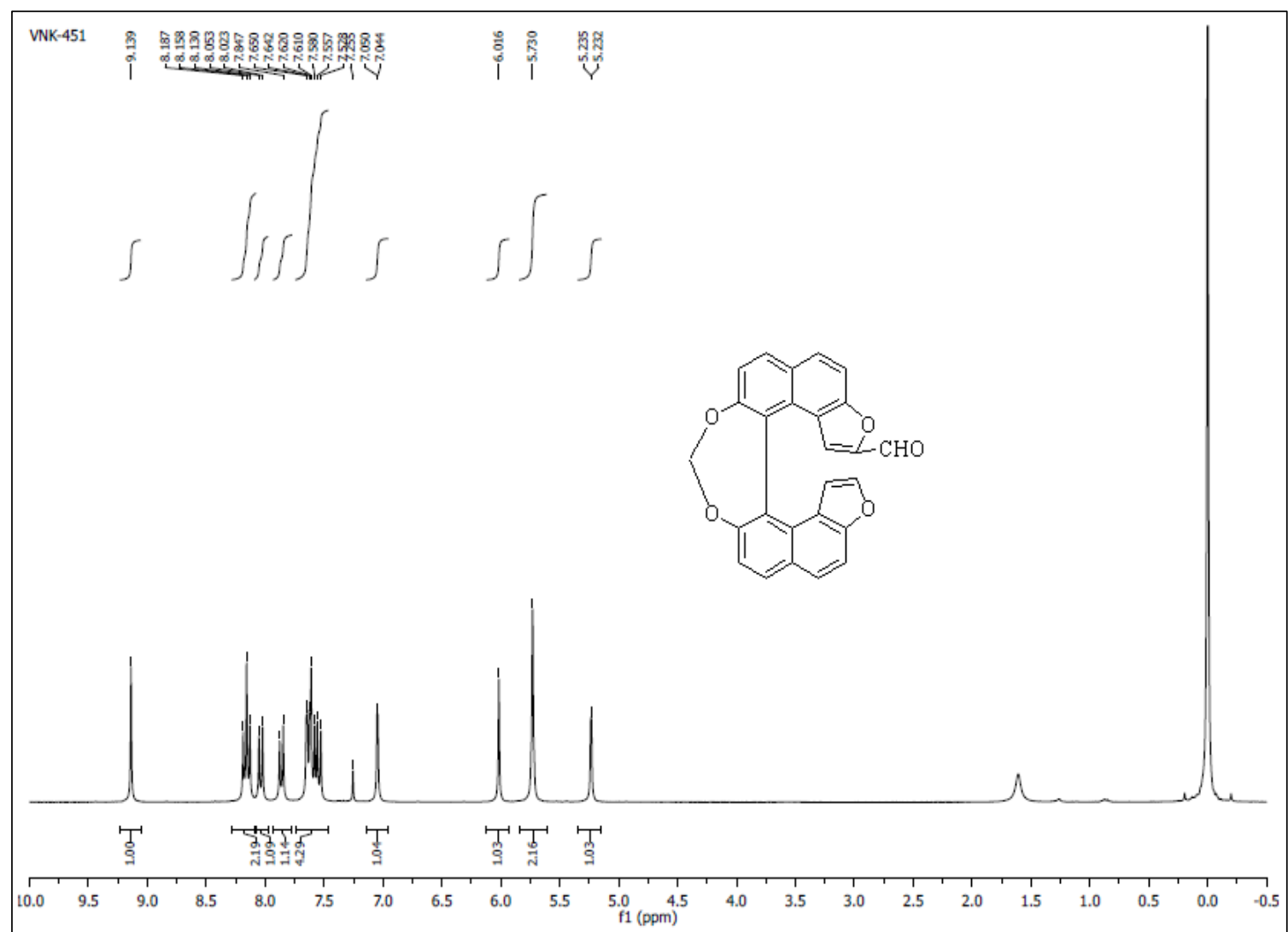

Figure S50. ${ }^{1} \mathrm{H}$ NMR (300 MHz, $\left.\mathrm{CDCl}_{3}, \mathrm{ppm}\right)$ helicenoid mono aldehyde, 11. 


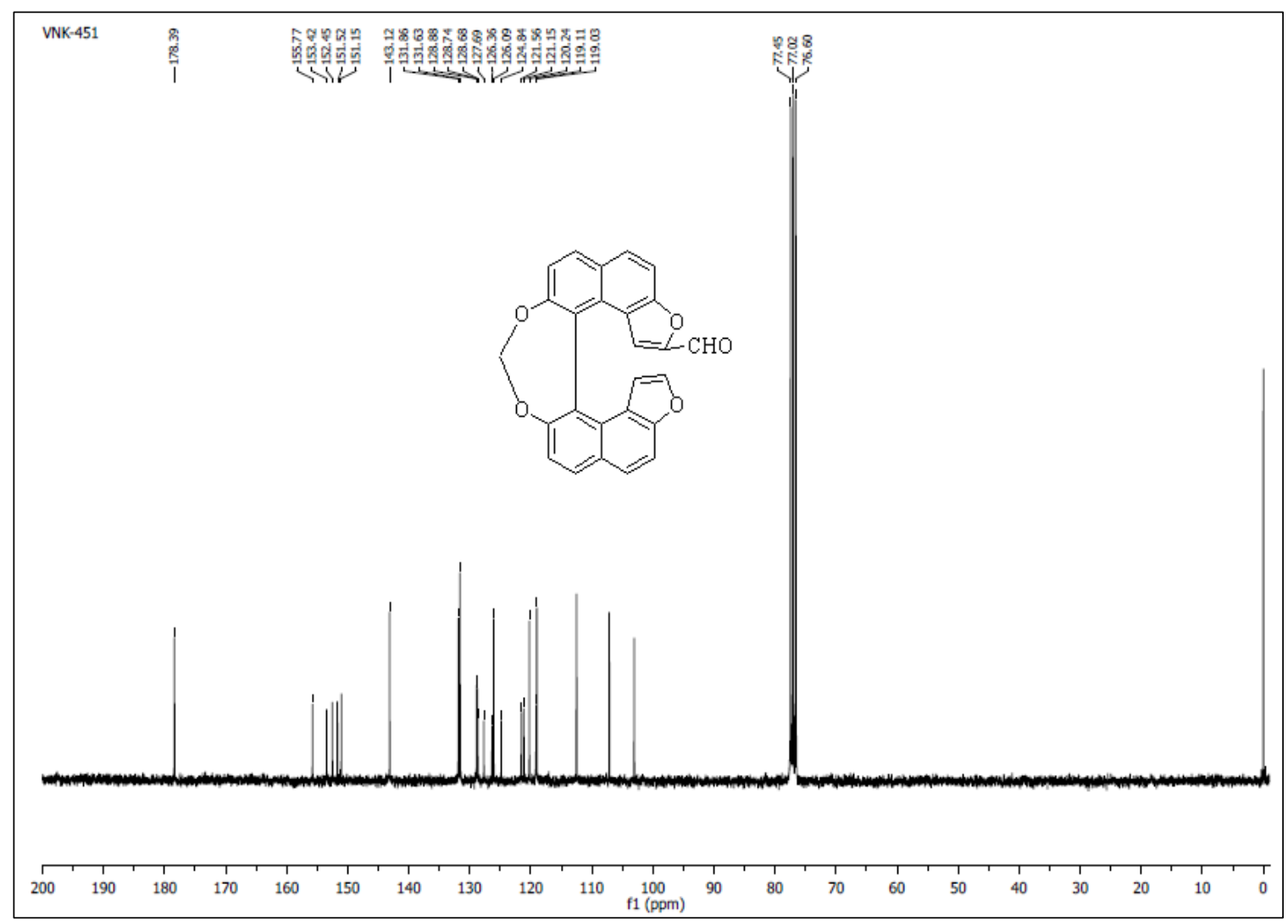

Figure S51. ${ }^{13} \mathrm{C}\left\{{ }^{1} \mathrm{H}\right\}$ NMR $\left(75 \mathrm{MHz}, \mathrm{CDCl}_{3}\right.$, ppm) helicenoid mono aldehyde, 11.

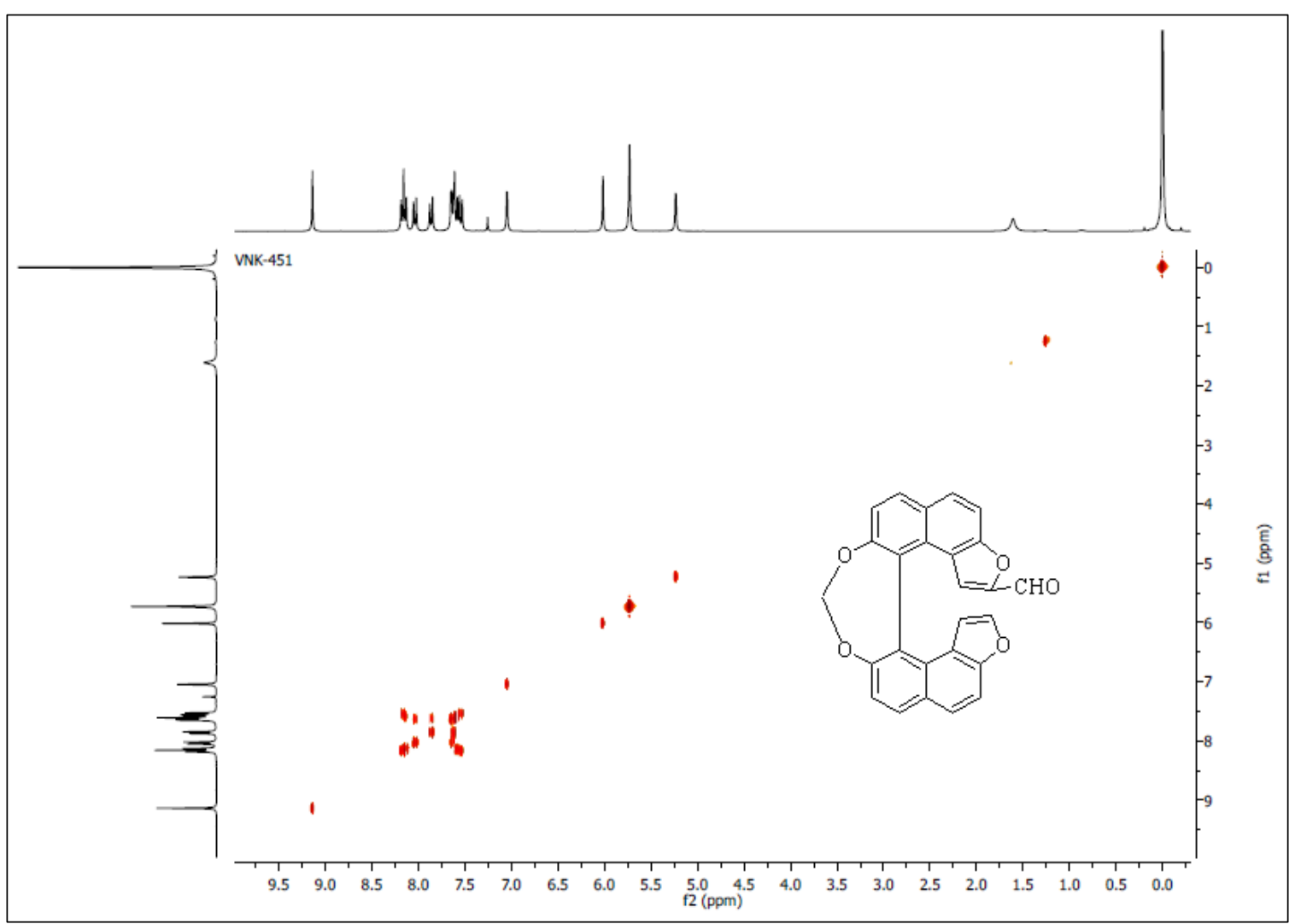

Figure S52. ${ }^{1} \mathrm{H}-{ }^{1} \mathrm{H} \operatorname{COSY}\left(300 \mathrm{MHz}, \mathrm{CDCl}_{3}, \mathrm{ppm}\right)$ helicenoid mono aldehyde, 11. 


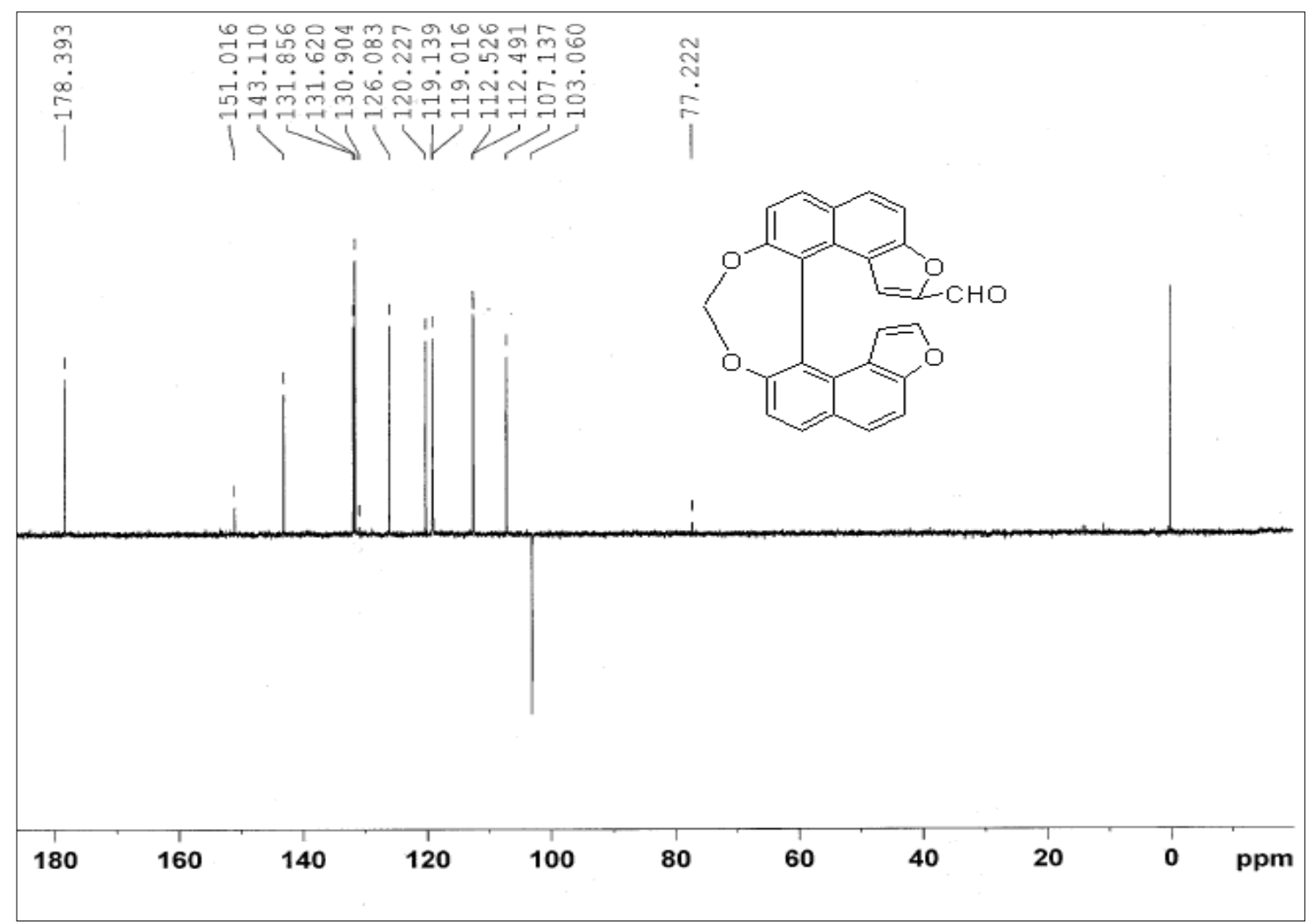

Figure S53. DEPT-135 NMR (75 MHz, $\mathrm{CDCl}_{3}$, ppm) helicenoid mono aldehyde, 11.

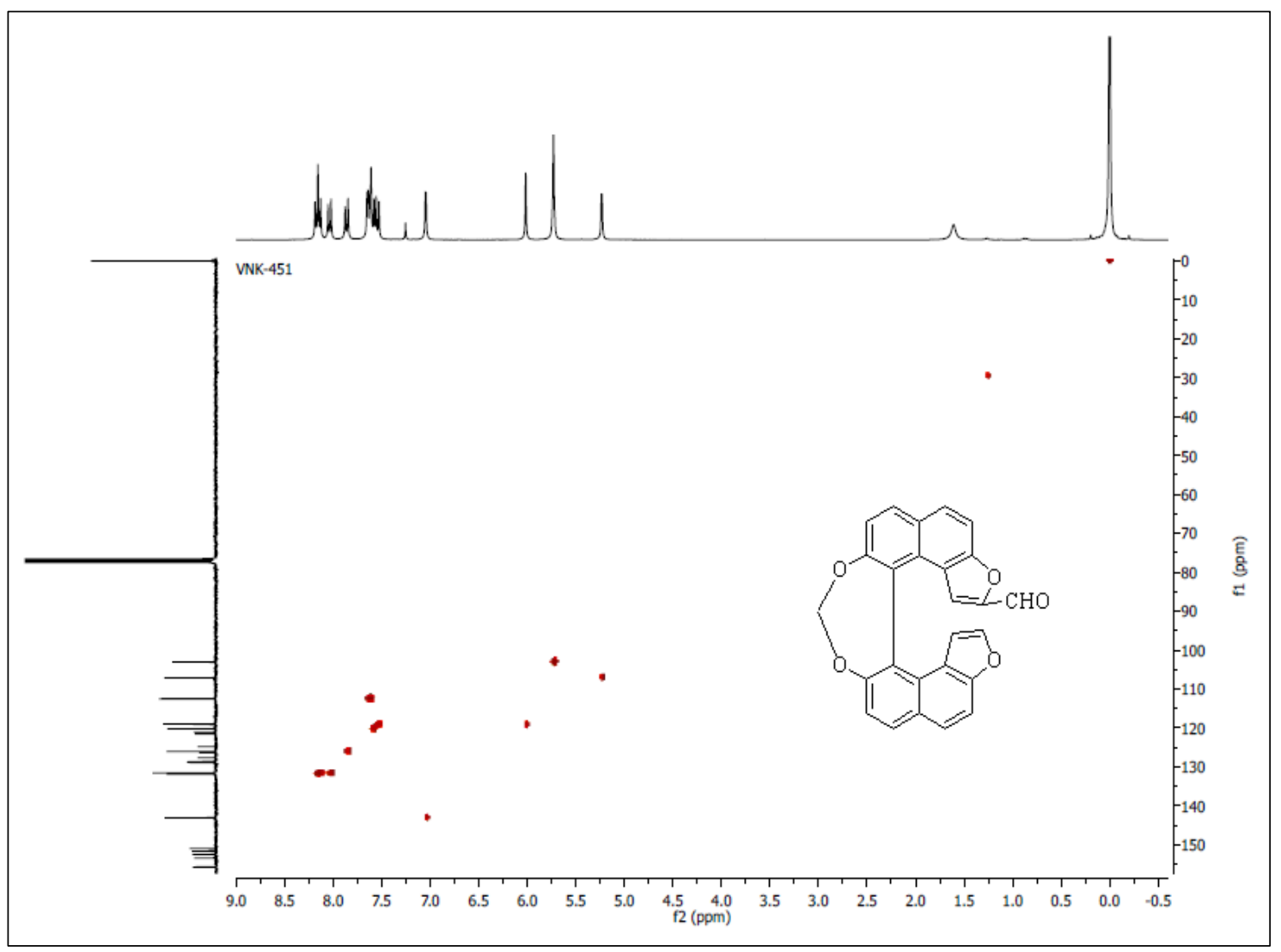

Figure S54. ${ }^{1} \mathrm{H}_{-}{ }^{13} \mathrm{C} \mathrm{HSQC}\left(300\right.$ and $\left.75 \mathrm{MHz}, \mathrm{CDCl}_{3}, \mathrm{ppm}\right)$ helicenoid mono aldehyde, 11. 


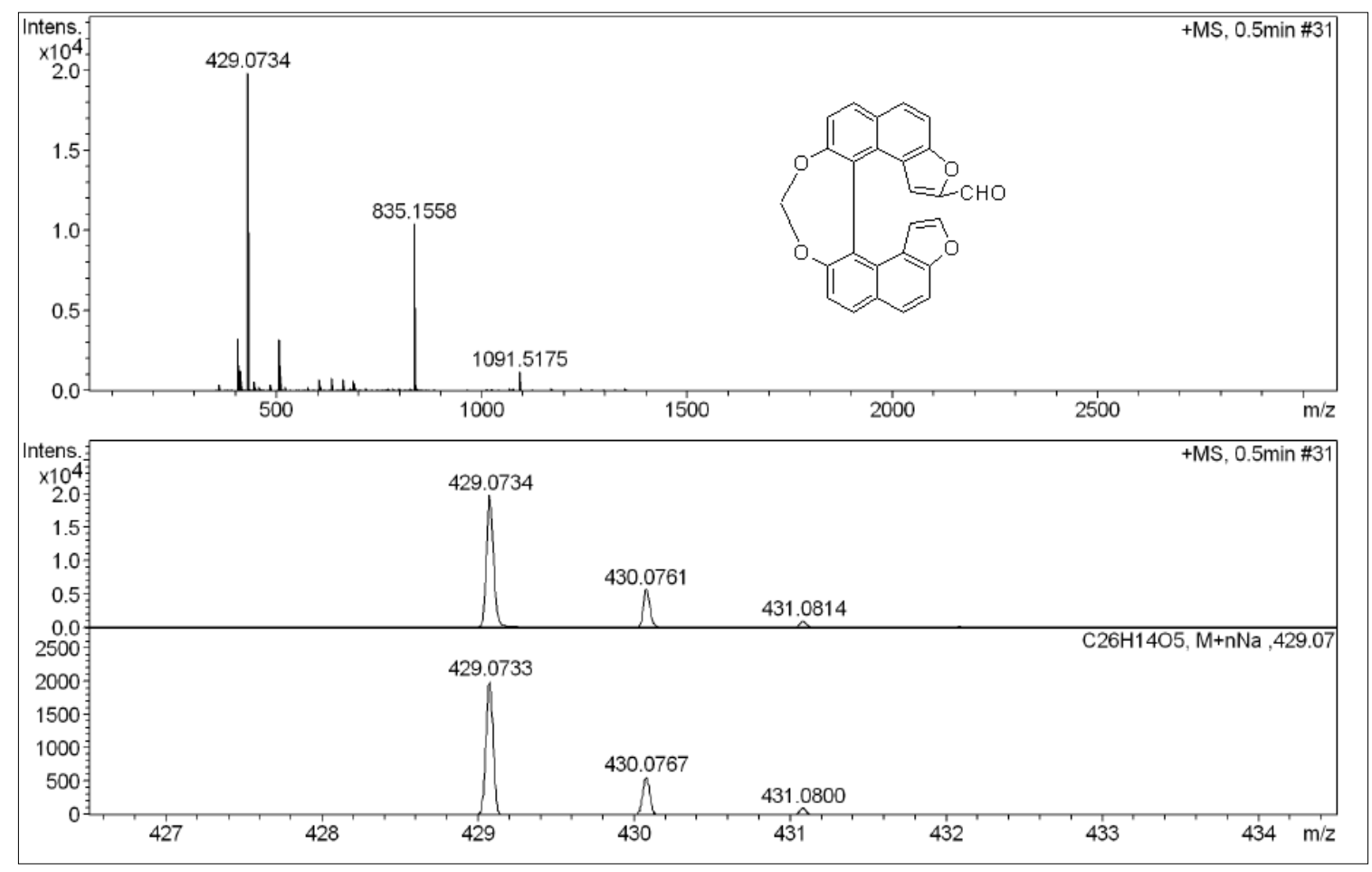

Figure S55. HRMS helicenoid mono aldehyde, 11.

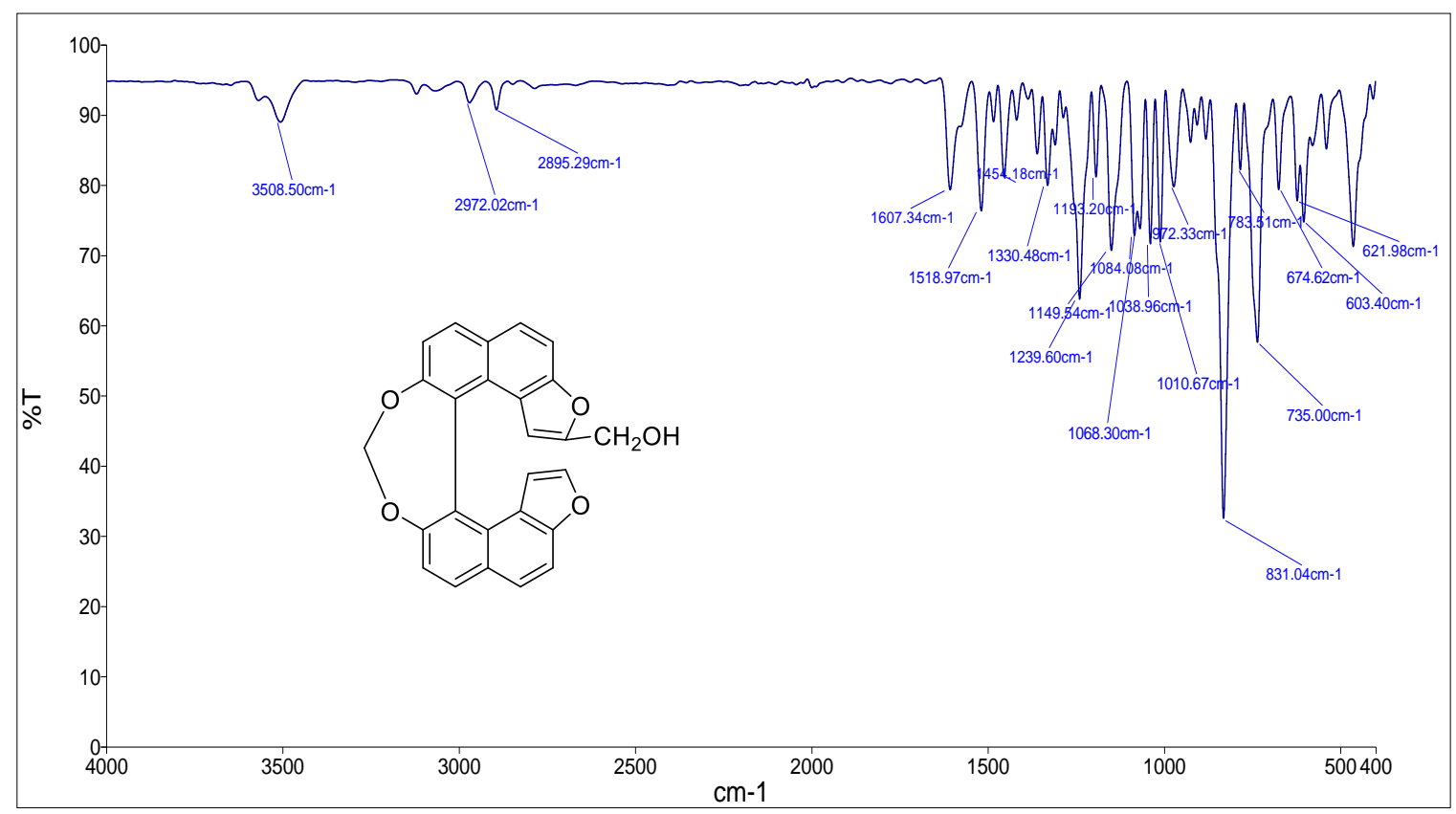

Figure S56. IR of helicenoid mono alcohol, 12. 


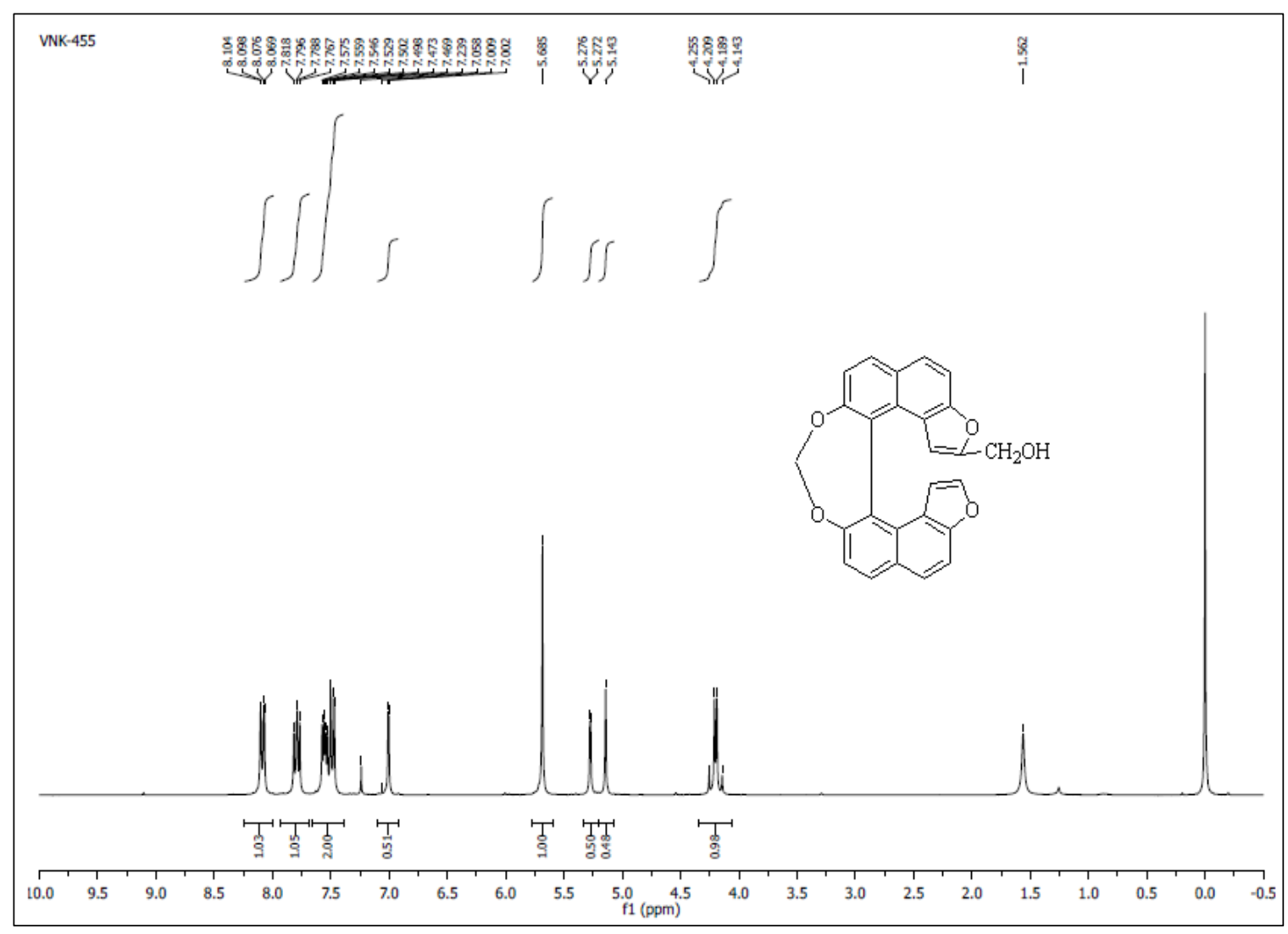

Figure S57. ${ }^{1} \mathrm{H}$ NMR (300 MHz, $\left.\mathrm{CDCl}_{3}, \mathrm{ppm}\right)$ of helicenoid mono alcohol, 12.

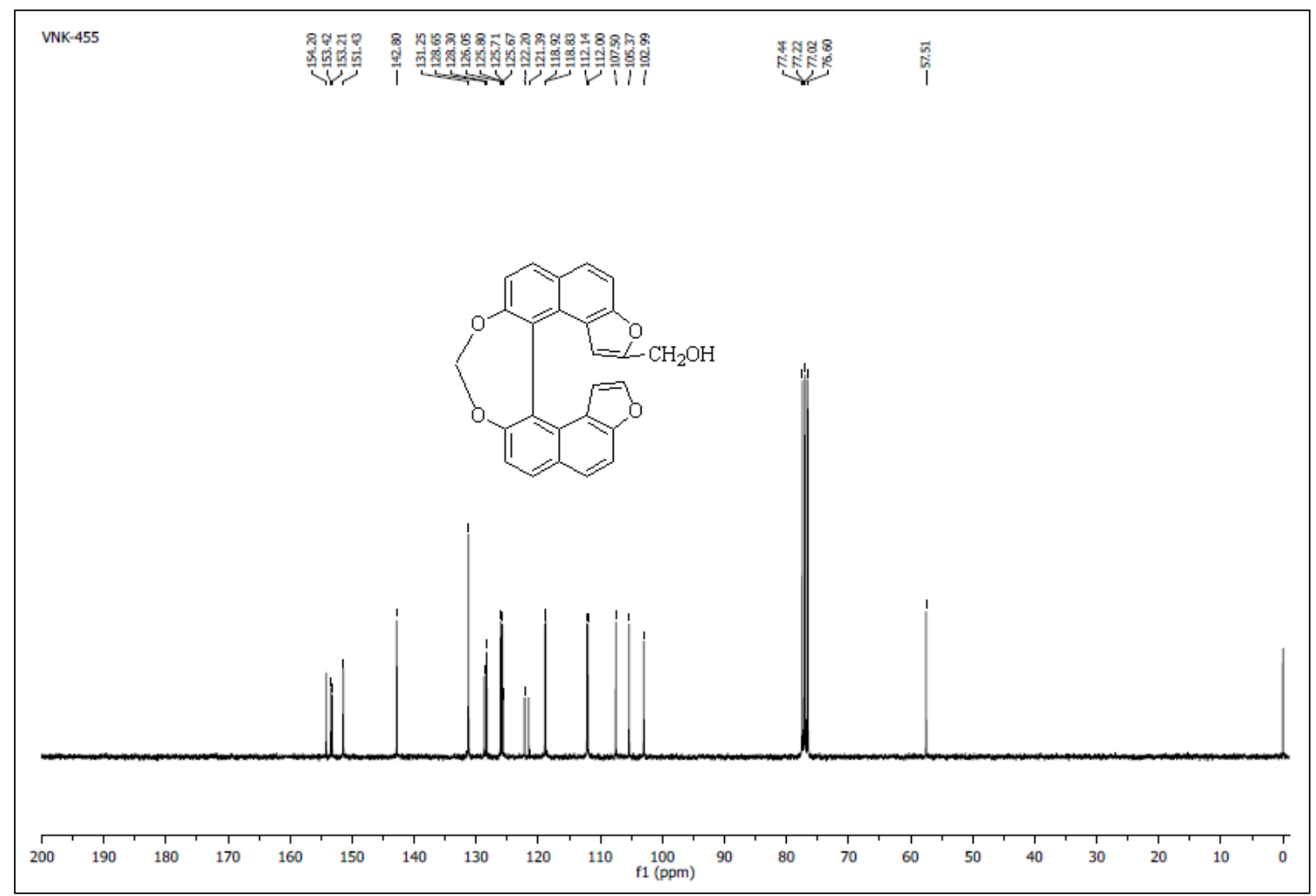

Figure S58. ${ }^{13} \mathrm{C}\left\{{ }^{1} \mathrm{H}\right\} \mathrm{NMR}\left(75 \mathrm{MHz}, \mathrm{CDCl}_{3}, \mathrm{ppm}\right)$ of helicenoid mono alcohol, 12. 


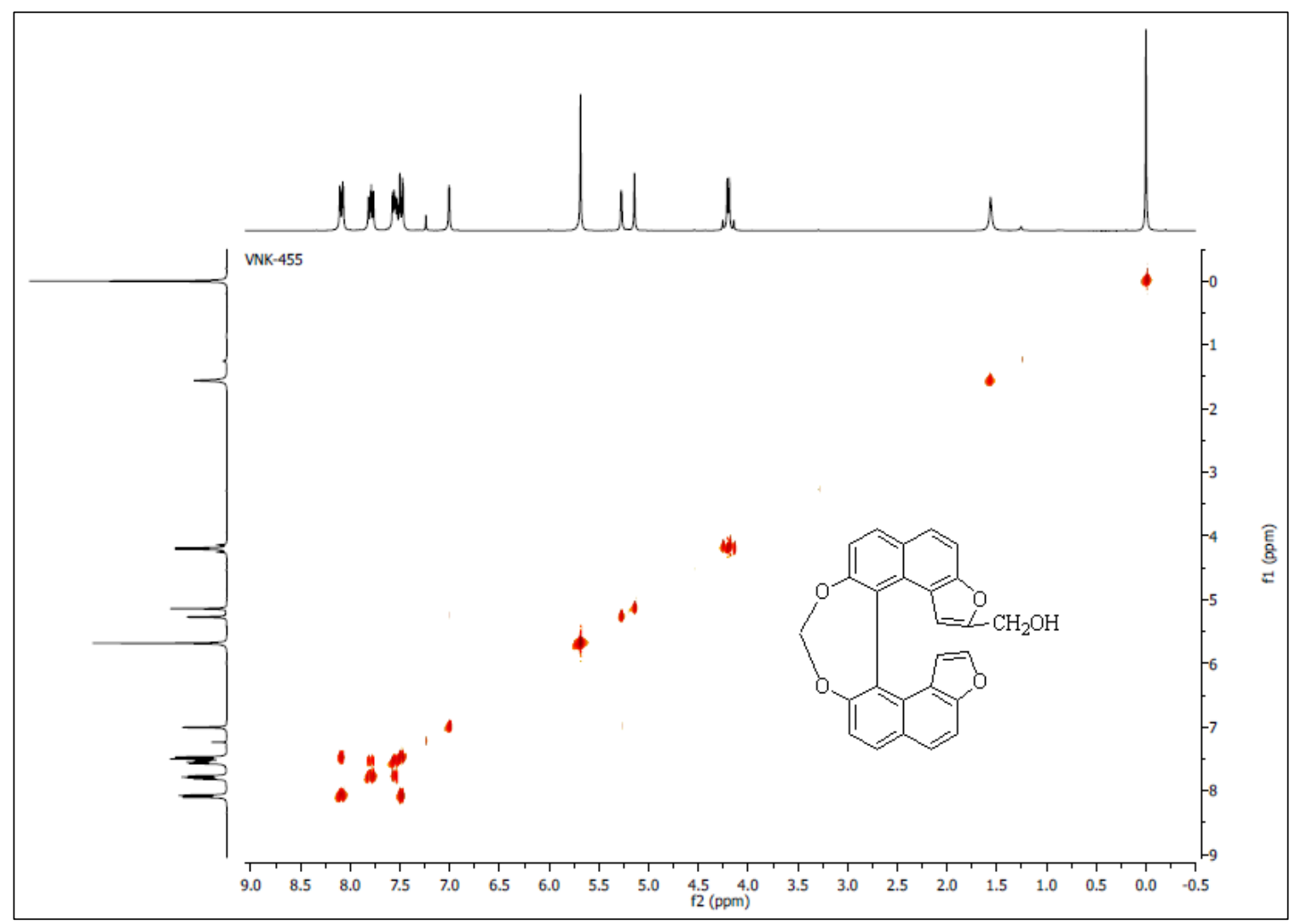

Figure S59. ${ }^{1} \mathrm{H}-{ }^{1} \mathrm{H}$ COSY $\left(300 \mathrm{MHz}, \mathrm{CDCl}_{3}\right.$, ppm) of helicenoid mono alcohol, 12.

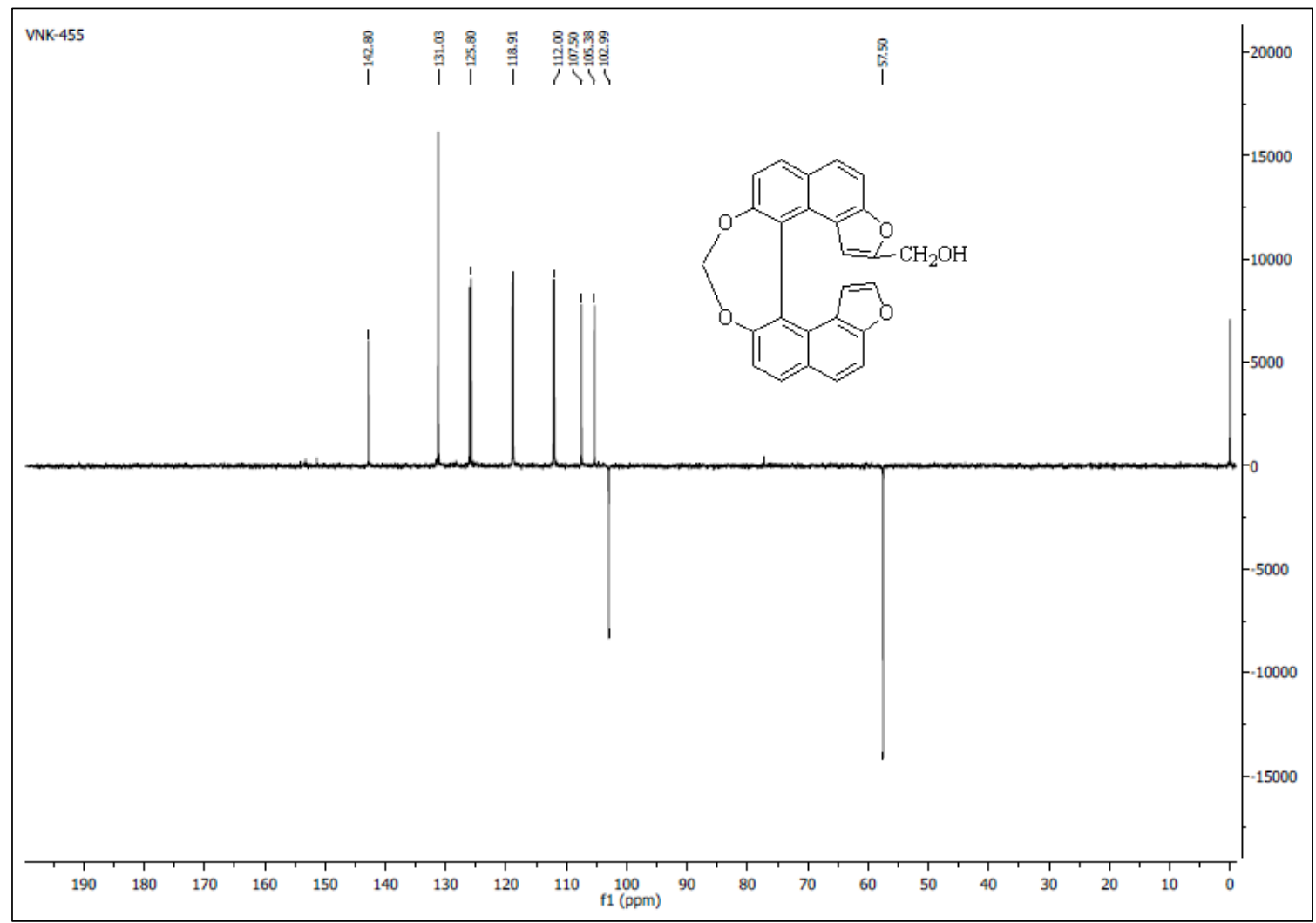

Figure S60. DEPT-135 NMR (75 MHz, $\left.\mathrm{CDCl}_{3}, \mathrm{ppm}\right)$ of helicenoid mono alcohol, 12. 


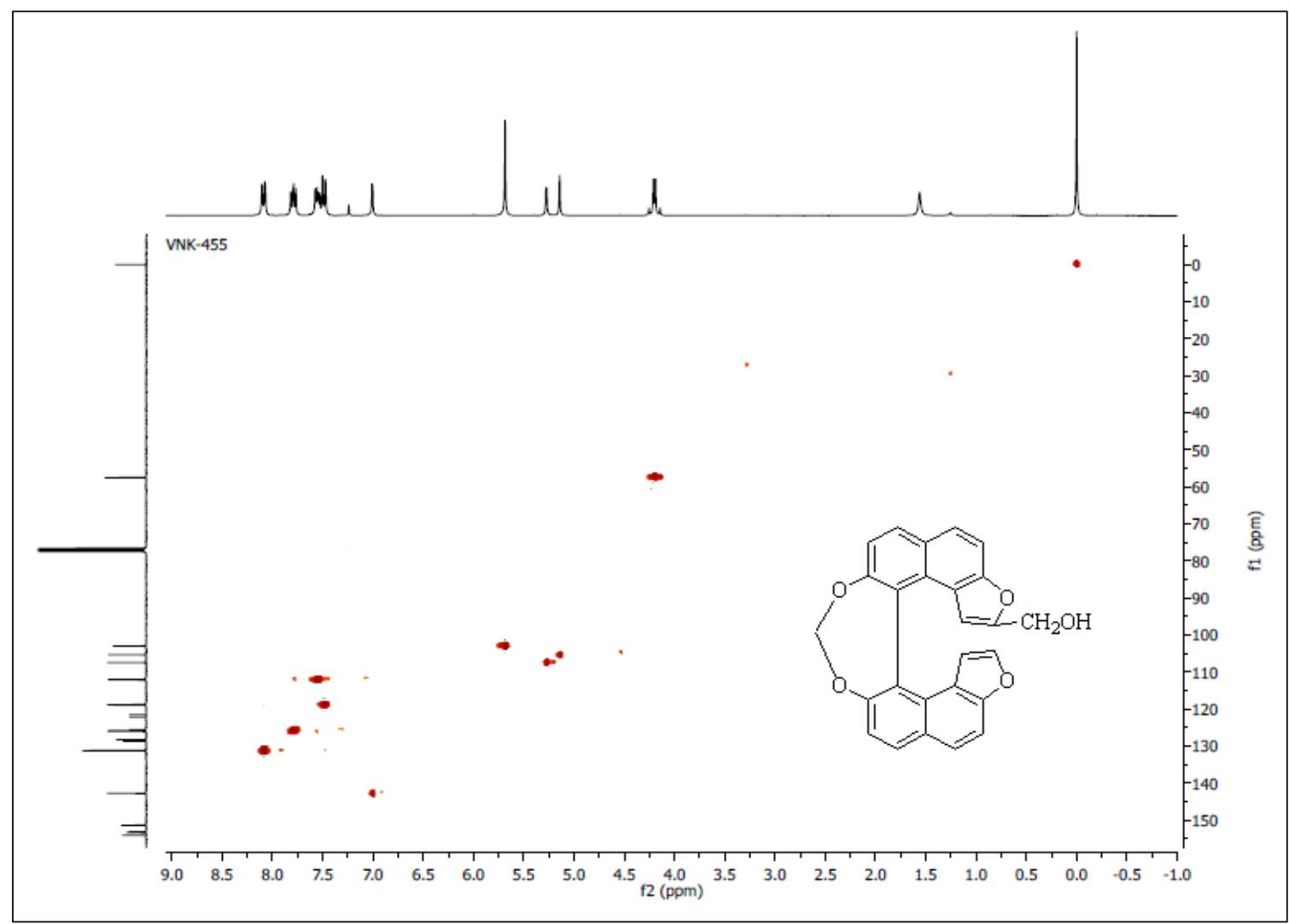

Figure S61. ${ }^{1} \mathrm{H}-{ }^{13} \mathrm{C}$ HSQC (300 and $75 \mathrm{MHz}, \mathrm{CDCl}_{3}$, ppm) of helicenoid mono alcohol, 12.

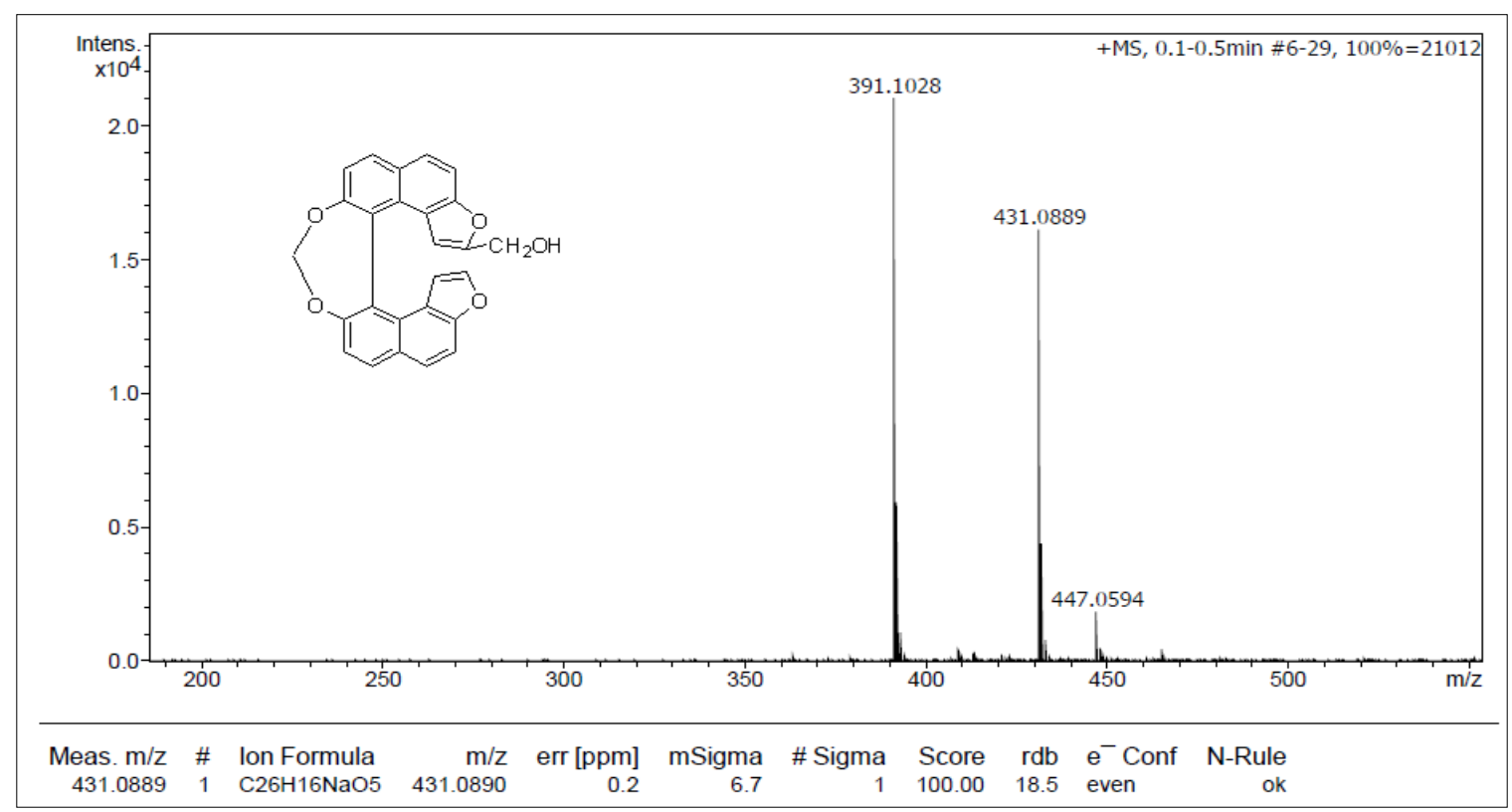

Figure S62. ${ }^{1}$ HRMS of helicenoid mono alcohol, 12. 


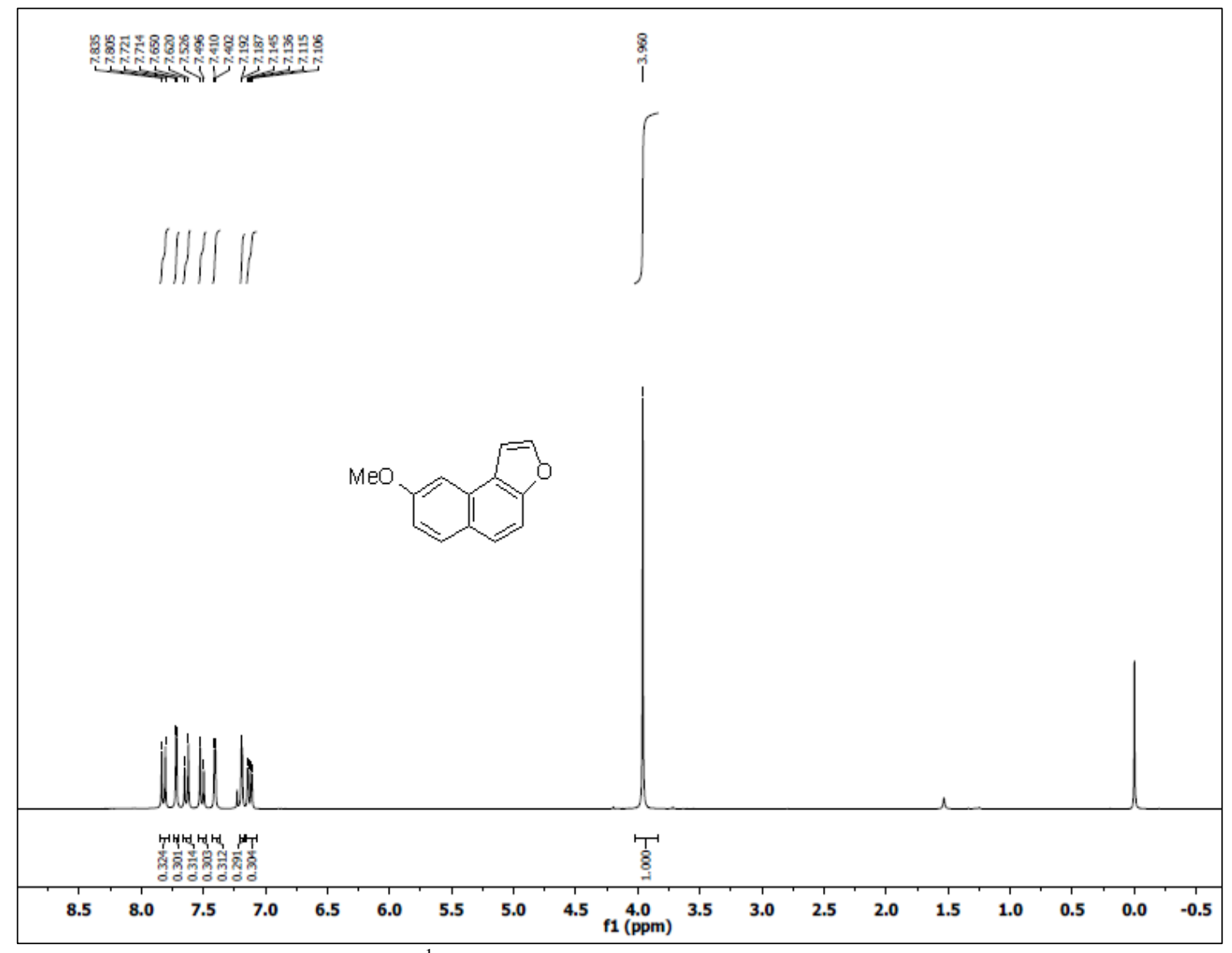

Figure S63. ${ }^{1}$ H NMR of 8-methoxynaphtho[2,1-b]furan, 14.

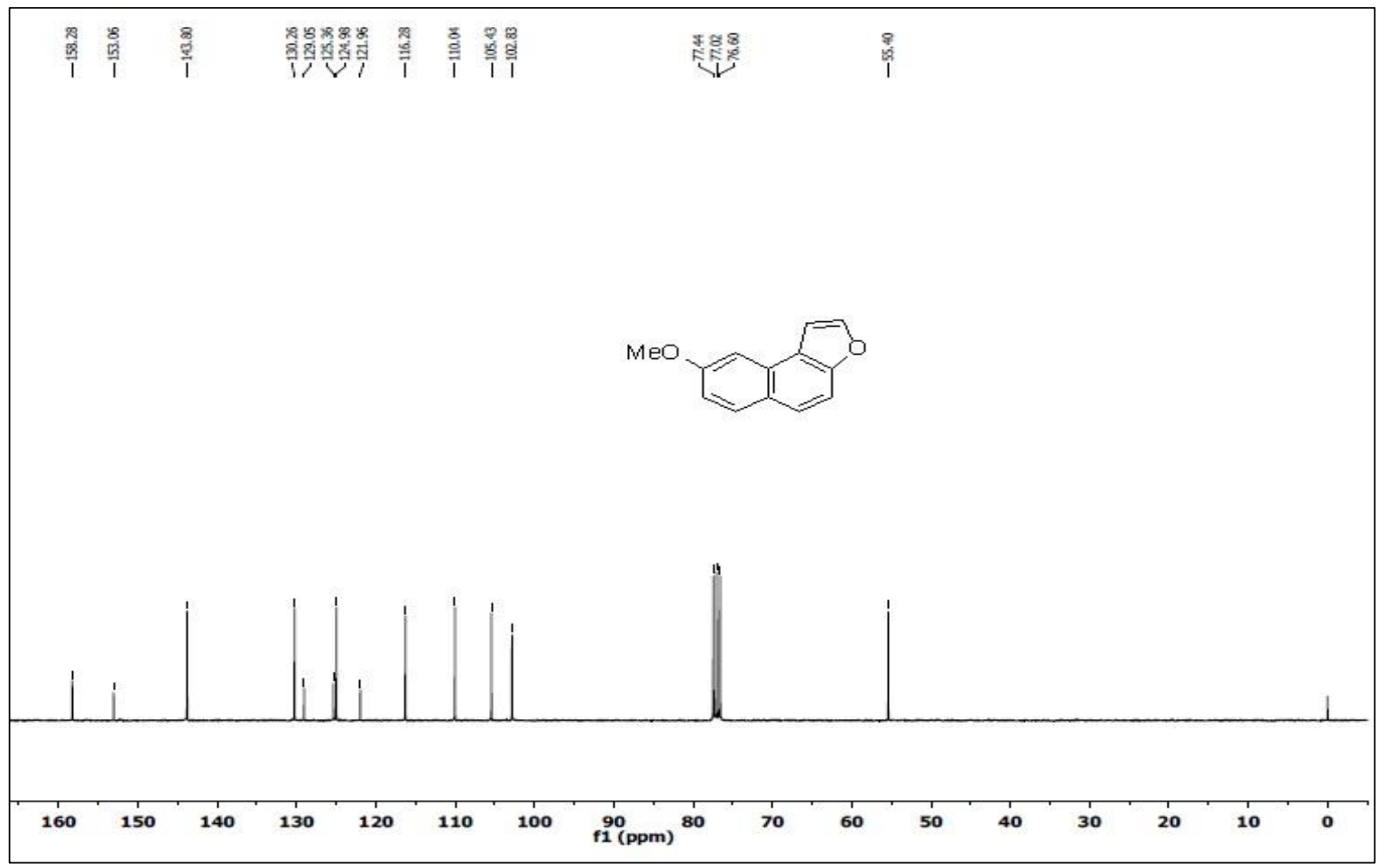

Figure S64. ${ }^{13} \mathrm{C}\left\{{ }^{1} \mathrm{H}\right\}$ NMR of 8-methoxynaphtho[2,1-b]furan, 14. 


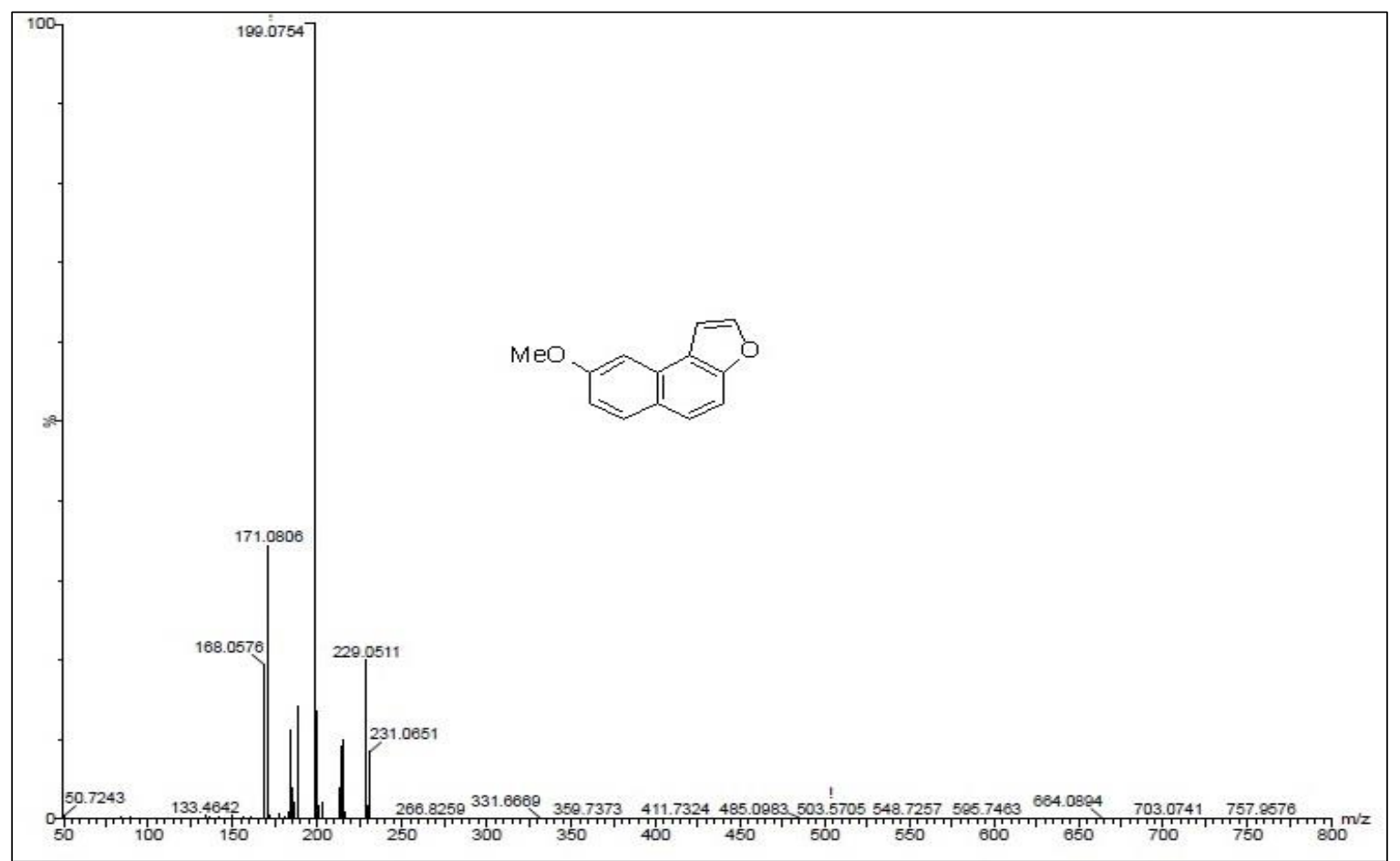

Figure S65. HRMS of 8-methoxynaphtho[2,1-b]furan, 14.

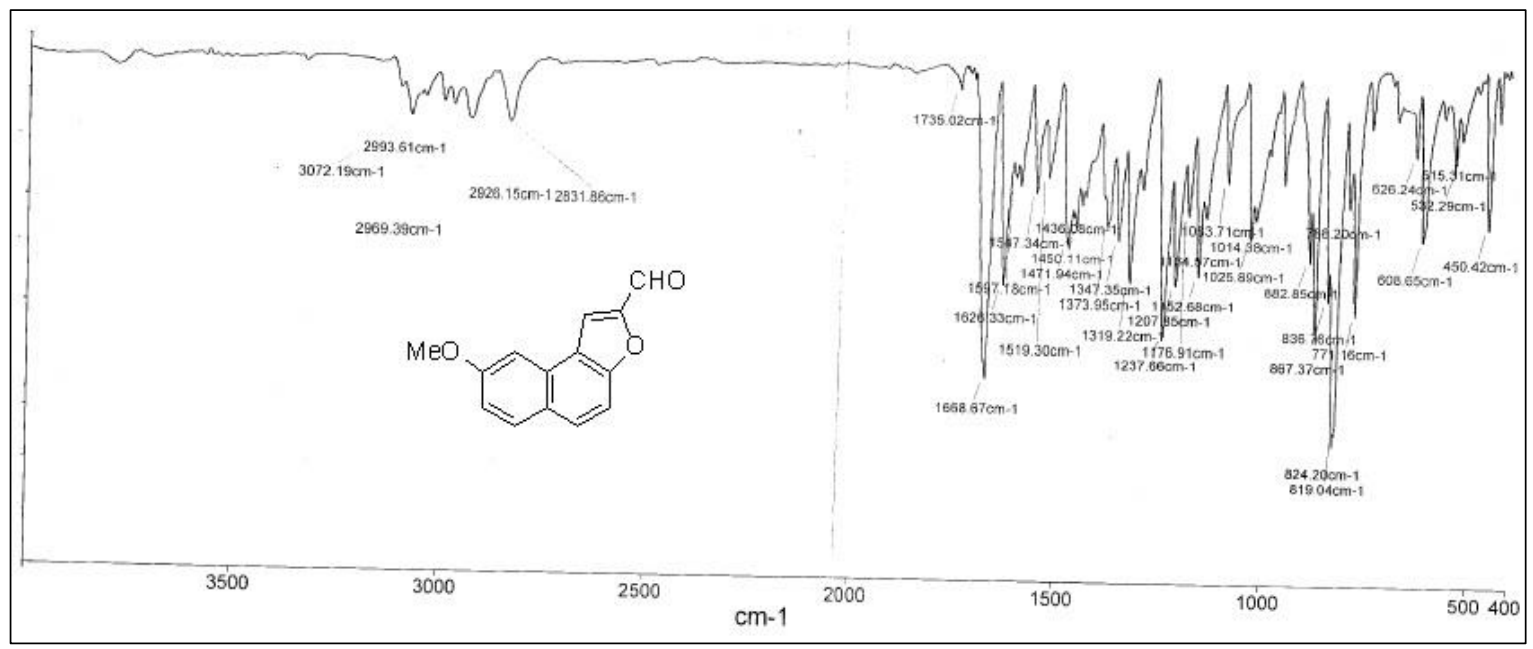

Figure S66. 8-methoxynaphtjo[2,1-b]furan-2-carbaldehyde, 15. 


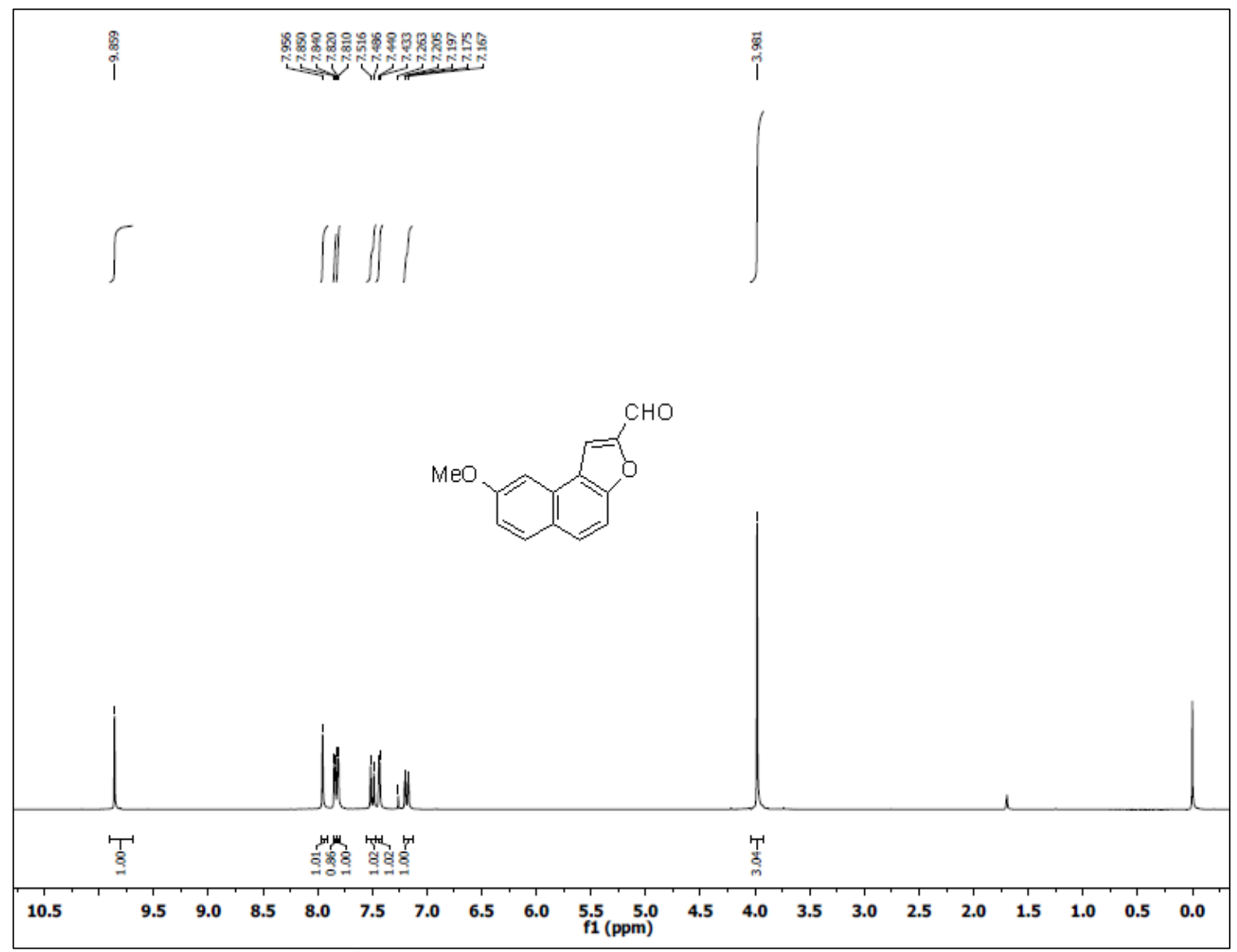

Figure S67. ${ }^{1}$ H NMR 8-methoxynaphtjo[2,1-b]furan-2-carbaldehyde, 15.

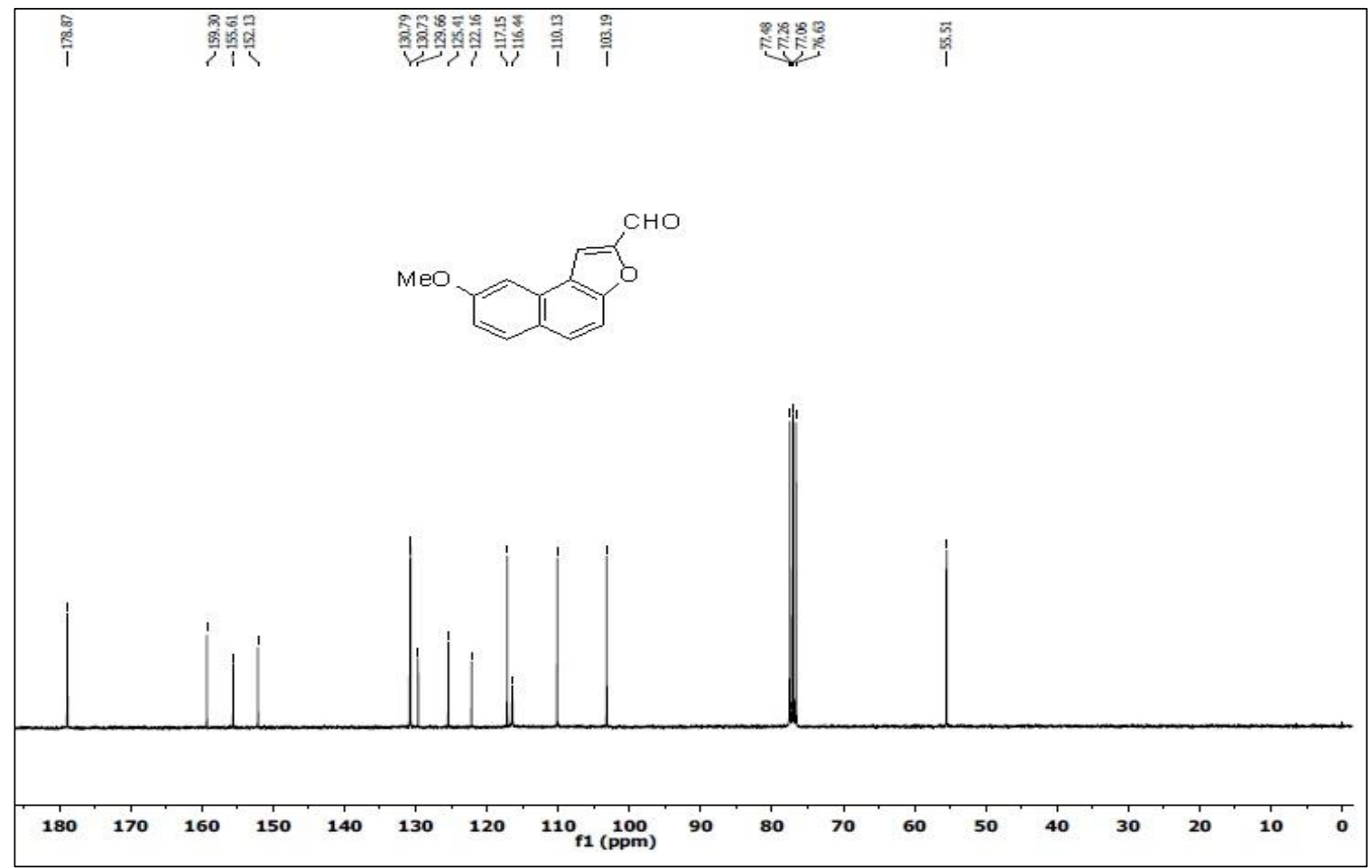

Figure S68. ${ }^{13} \mathrm{C}\left\{{ }^{1} \mathrm{H}\right\}$ NMR 8-methoxynaphtjo[2,1-b]furan-2-carbaldehyde, 15. 


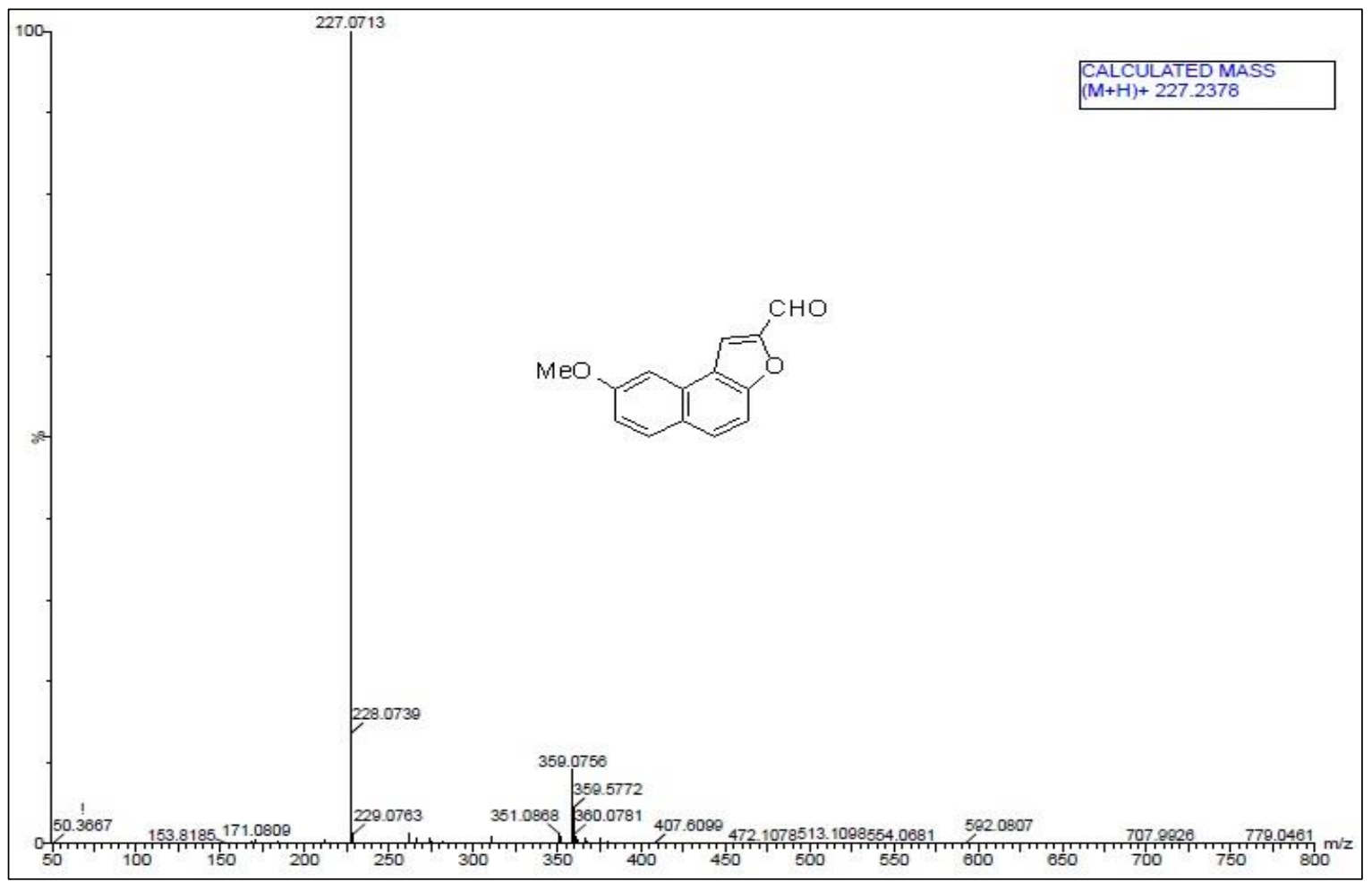

Figure S69. HRMS 8-methoxynaphtjo[2,1-b]furan-2-carbaldehyde, 15. 
Single Crystal $X$-ray diffraction analysis

Crystal structure of 7 (CCDC 1401513)

A suitable single crystal of $(P)$-helicenoid dialdehyde, 7 was gradually developed by the slow vapour diffusion method in chloroform and hexane solvents.

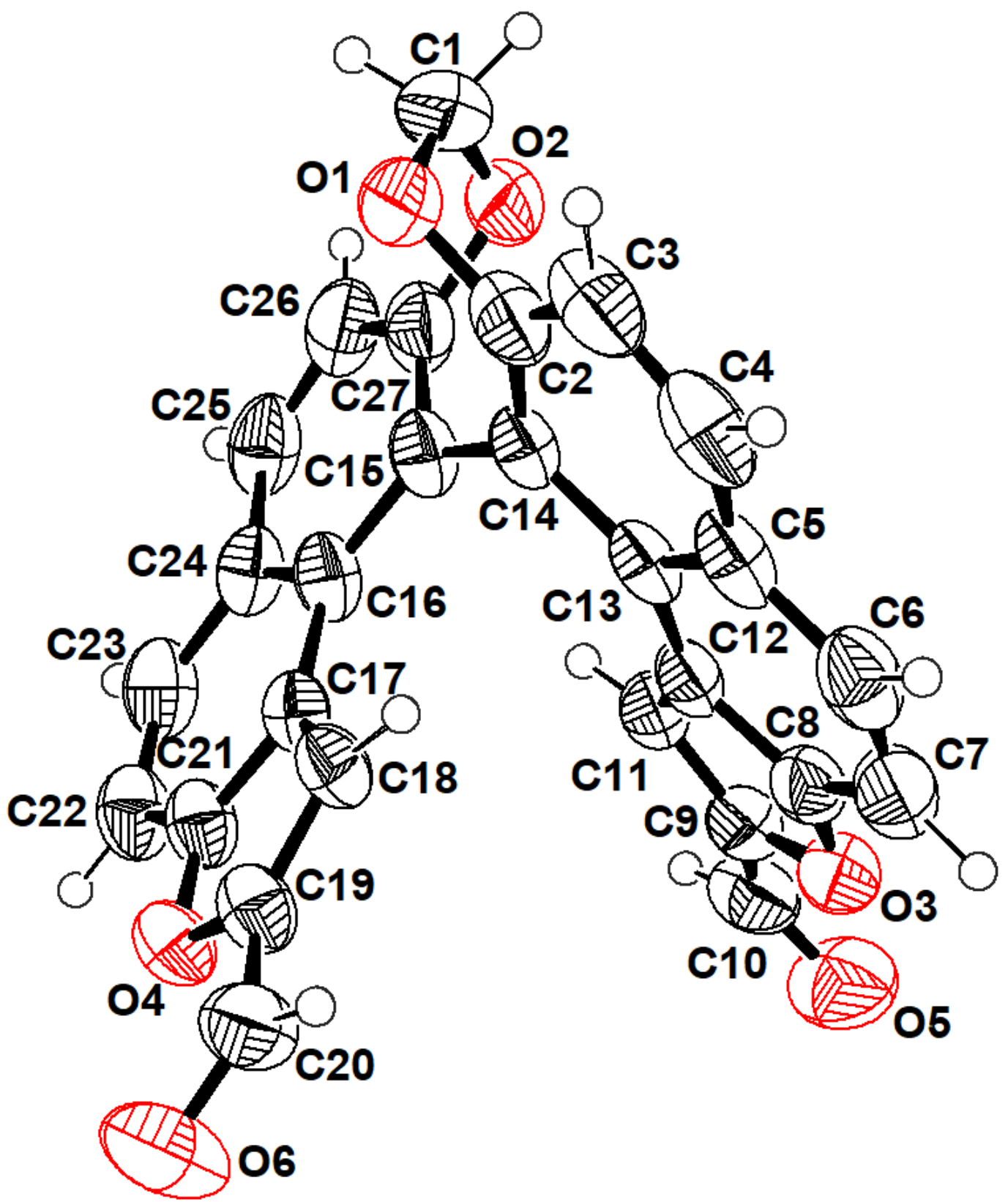

Figure S70. ORTEP diagram of helicenoid dialdehde, 7. Atomic displacement ellipsoids are drawn at 50\% probability level 
Table S1. Crystal data

\begin{tabular}{|c|c|}
\hline Identification code & Shelx \\
\hline Empirical formula & $\mathrm{C}_{27} \mathrm{H}_{14} \mathrm{O}_{6}$ \\
\hline Formula weight & 434.38 \\
\hline Temperature & $150(2) \mathrm{K}$ \\
\hline Wavelength & $1.5418 \mathrm{~A}$ \\
\hline Crystal system, space group & Monoclinic, $\mathrm{P} 21$ \\
\hline Unit cell dimensions & $\begin{array}{l}\mathrm{a}=20.336(3) \text { A } \quad \text { alpha }=90 \mathrm{deg} . \\
\mathrm{b}=7.2466(2) \mathrm{A} \quad \text { beta }=140.71(3) \mathrm{deg} . \\
\mathrm{c}=10.7546(14) \text { A } \quad \text { gamma }=90 \mathrm{deg} .\end{array}$ \\
\hline Volume & $1003.64(18) \mathrm{A}^{\wedge} 3$ \\
\hline Z, Calculated density & $2,1.437 \mathrm{Mg} / \mathrm{m}^{\wedge} 3$ \\
\hline Absorption coefficient & $0.849 \mathrm{~mm}^{\wedge}-1$ \\
\hline $\mathbf{F}(\mathbf{0 0 0})$ & 448 \\
\hline Crystal size & $0.32 \times 0.28 \times 0.24 \mathrm{~mm}$ \\
\hline Theta range for data collection & 3.43 to $71.28 \mathrm{deg}$. \\
\hline Limiting indices & $-22<=\mathrm{h}<=24,-8<=\mathrm{k}<=5,-13<=\mathrm{l}<=12$ \\
\hline Reflections collected / unique & $5456 / 2039[\mathrm{R}(\mathrm{int})=0.0683]$ \\
\hline Completeness to theta $=71.28$ & $96.7 \%$ \\
\hline Absorption correction & Semi-empirical from equivalents \\
\hline Max. and min. transmission & 0.8222 and 0.7728 \\
\hline Refinement method & Full-matrix least-squares on $\mathrm{F}^{\wedge} 2$ \\
\hline Data / restraints / parameters & 2039 / 1 / 299 \\
\hline Goodness-of-fit on $F^{\wedge} 2$ & 1.191 \\
\hline Final $R$ indices $[I>2 \operatorname{sigma}(I)]$ & $\mathrm{R} 1=0.0979, \mathrm{wR} 2=0.2193$ \\
\hline $\mathbf{R}$ indices (all data) & $\mathrm{R} 1=0.1101, \mathrm{wR} 2=0.2588$ \\
\hline Absolute structure parameter & $10(10)$ \\
\hline Extinction coefficient & $0.034(7)$ \\
\hline Largest diff. peak and hole & 1.026 and -0.989 e. $\mathrm{A}^{\wedge}-3$ \\
\hline
\end{tabular}




\section{HPLC Graphs}

1 For furo fused BINOL, 1:

$\begin{array}{ll}\text { Column } & : \text { Chiralcel® OD }[4.6 \mathrm{~mm} \text { i.d. } \times 250 \mathrm{~mm}] \\ \text { Mobile Phase } & : \text { Hexane: } i-\operatorname{PrOH}(50: 50) \\ \text { Flow rate } & : 0.5 \mathrm{ml} / \mathrm{min} \\ \text { Detector } & : \mathrm{UV}(\lambda=300 \mathrm{~nm}) \\ \text { Temperature } & : 20^{\circ} \mathrm{C}\end{array}$

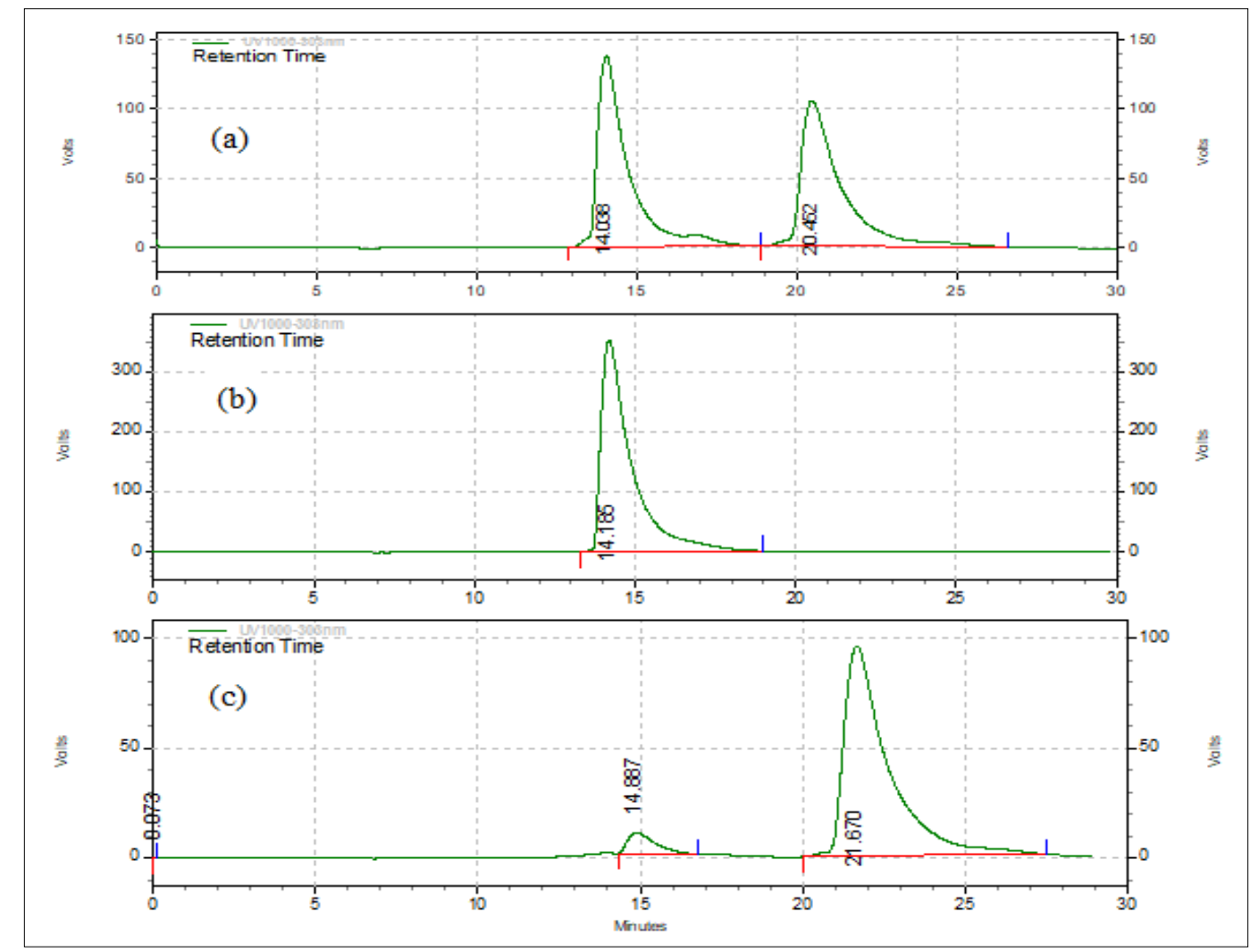

\begin{tabular}{|c|c|c|c|c|c|}
\hline \multicolumn{2}{|c|}{$r a c-1$} & \multicolumn{2}{c|}{$(R)-1$} & \multicolumn{2}{c|}{$(S)-1$} \\
\hline $\begin{array}{c}\text { Retention } \\
\text { Time }\end{array}$ & Area \% & $\begin{array}{c}\text { Retention } \\
\text { Time }\end{array}$ & Area \% & $\begin{array}{c}\text { Retention } \\
\text { Time }\end{array}$ & Area \% \\
\hline 14.038 & 50.60 & 14.185 & 100.00 & 14.887 & 5.90 \\
\hline 20.452 & 49.40 & - & - & 21.670 & 94.09 \\
\hline
\end{tabular}

Figure S71. HPLC chromatograms for, (a) rac-1; (b) (R)-1; (c) (S)-1. 
2. Helicenoid, 2:

Column

Mobile Phase

Flow rate

Detector

Temperature
: Chiralcel® IA [4.6 mm i.d. x $250 \mathrm{~mm}$ ]

: Hexane: DCM 97:03

: $0.5 \mathrm{ml} / \mathrm{min}$

: $\operatorname{UV}(\lambda=313 \mathrm{~nm})$

: $20^{\circ} \mathrm{C}$
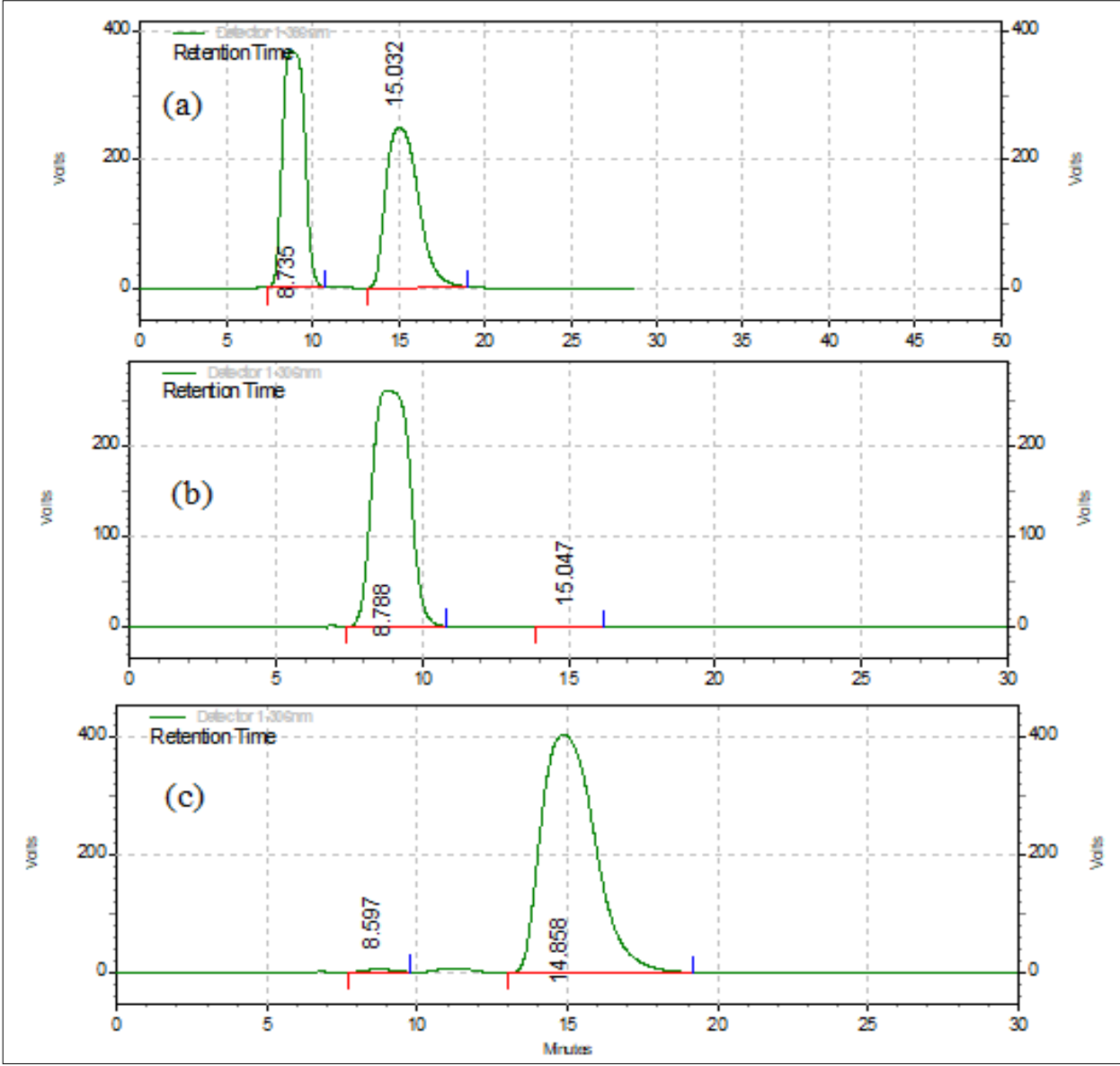

\begin{tabular}{|c|c|c|c|c|c|}
\hline \multicolumn{2}{|c|}{$r a c-2$} & \multicolumn{2}{c|}{$(P)-2$} & \multicolumn{2}{c|}{$(M)$-2 } \\
\hline $\begin{array}{c}\text { Retention } \\
\text { Time }\end{array}$ & Area \% & $\begin{array}{c}\text { Retention } \\
\text { Time }\end{array}$ & Area \% & $\begin{array}{c}\text { Retention } \\
\text { Time }\end{array}$ & Area \% \\
\hline 8.73 & 49.99 & 8.78 & 99.69 & 8.59 & 0.66 \\
\hline 15.03 & 50.01 & 15.04 & 0.31 & 14.85 & 98.91 \\
\hline
\end{tabular}

Figure S72. HPLC chromatograms for, (a) rac-2; (b) (P)-2; (c) (M)-1. 
3. For dibromo helicenoid, 3:

Column

Mobile Phase

Flow rate

Detector

Temperature
: Chiralcel® IA [4.6 mm i.d. x $250 \mathrm{~mm}$ ]

: Hexane: DCM 97:03

: $0.5 \mathrm{ml} / \mathrm{min}$

: $\mathrm{UV}(\lambda=314 \mathrm{~nm})$

: $20^{\circ} \mathrm{C}$
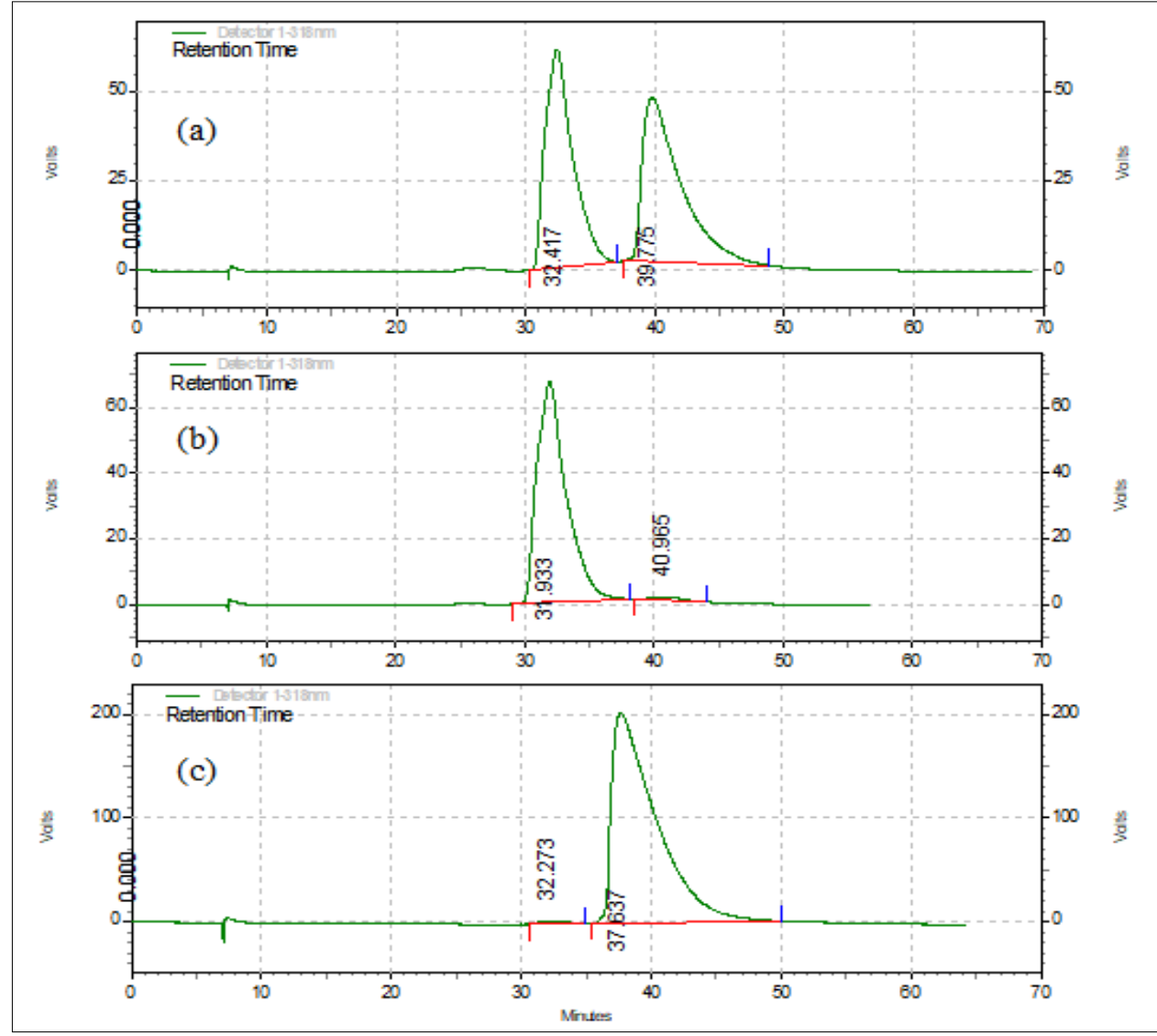

\begin{tabular}{|c|c|c|c|c|c|}
\hline \multicolumn{2}{|c|}{$r a c-3$} & \multicolumn{2}{c|}{$(P)-3$} & \multicolumn{2}{c|}{$(M)$-3 } \\
\hline $\begin{array}{c}\text { Retention } \\
\text { Time }\end{array}$ & Area \% & $\begin{array}{c}\text { Retention } \\
\text { Time }\end{array}$ & Area \% & $\begin{array}{c}\text { Retention } \\
\text { Time }\end{array}$ & Area \% \\
\hline 32.41 & 48.75 & 31.93 & 98.28 & 32.27 & 0.83 \\
\hline 39.77 & 51.25 & 40.96 & 1.72 & 37.63 & 99.17 \\
\hline
\end{tabular}

Figure S73. HPLC chromatograms for, (a) rac-3; (b) (P)-3; (c) (M)-3. 
4. Helicenoid mono bromo cyanide, 4:

Column

Mobile Phase

Flow rate

Detector

Temperature
: Chiralcel® AD [4.6 mm i.d. x $250 \mathrm{~mm}$ ]

: Hexane: IPA 90:10

: $0.5 \mathrm{ml} / \mathrm{min}$

: $\mathrm{UV}(\lambda=318 \mathrm{~nm})$

: $20^{\circ} \mathrm{C}$

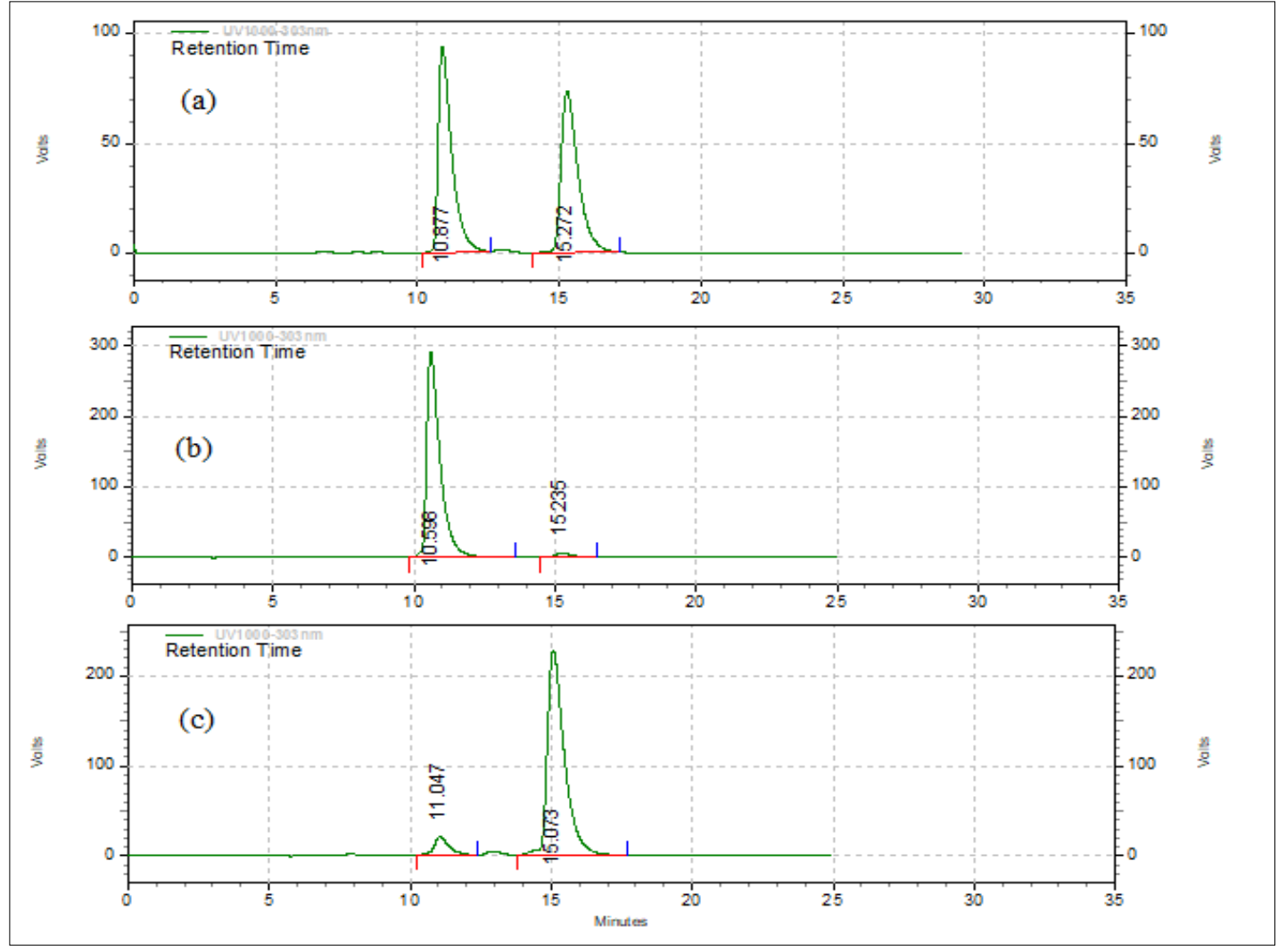

\begin{tabular}{|c|c|c|c|c|c|}
\hline \multicolumn{2}{|c|}{} & \multicolumn{2}{c|}{$(P)-4$} & \multicolumn{2}{c|}{$(M)-4$} \\
\hline $\begin{array}{c}\text { Retention } \\
\text { Time }\end{array}$ & Area \% & $\begin{array}{c}\text { Retention } \\
\text { Time }\end{array}$ & Area \% & $\begin{array}{c}\text { Retention } \\
\text { Time }\end{array}$ & Area \% \\
\hline 10.877 & 50.18 & 10.598 & 97.44 & 11.047 & 6.97 \\
\hline 15.272 & 49.82 & 15.235 & 2.56 & 15.073 & 93.03 \\
\hline
\end{tabular}

Figure S74. HPLC chromatograms for, (a) rac-4; (b) (P)-4; (c) (M)-4. 
5. For 2,16-distyryl helicenoid, 6:

Column

Mobile Phase

Flow rate

Detector

Temperature
: Chiralcel® IA [4.6 mm i.d. x $250 \mathrm{~mm}$ ]

: Hexane: IPA (90 10)

: $0.5 \mathrm{ml} / \mathrm{min}$

: $\operatorname{UV}(\lambda=359 \mathrm{~nm})$

: $20^{\circ} \mathrm{C}$

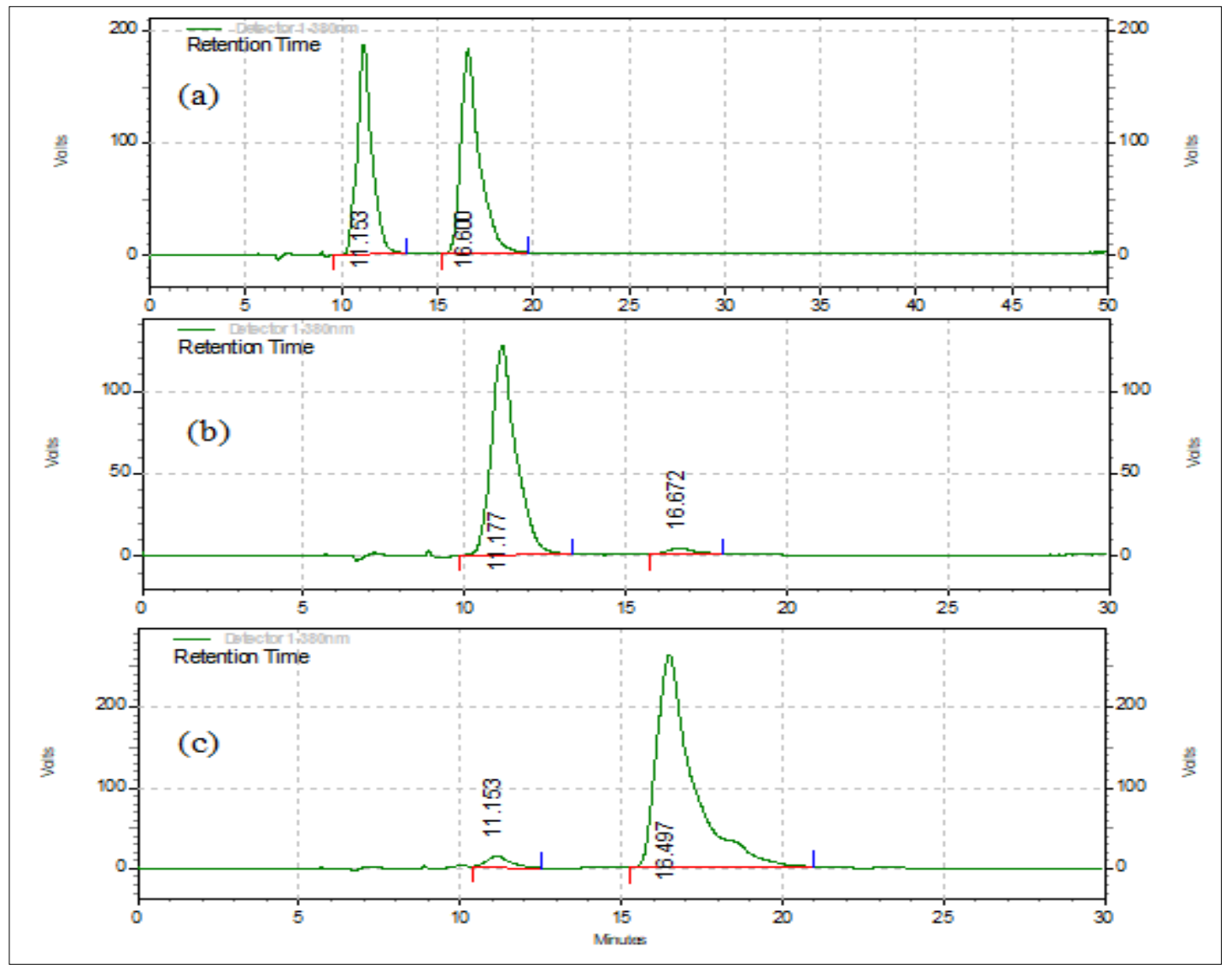

\begin{tabular}{|c|c|c|c|c|c|}
\hline \multicolumn{2}{|c|}{$r a c-6$} & \multicolumn{2}{c|}{$(P)-6$} & \multicolumn{2}{c|}{$(M)-6$} \\
\hline $\begin{array}{c}\text { Retention } \\
\text { Time }\end{array}$ & Area \% & $\begin{array}{c}\text { Retention } \\
\text { Time }\end{array}$ & Area \% & $\begin{array}{c}\text { Retention } \\
\text { Time }\end{array}$ & Area \% \\
\hline 11.15 & 44.83 & 11.17 & 96.77 & 11.15 & 3.15 \\
\hline 16.60 & 55.17 & 16.67 & 3.23 & 16.49 & 96.85 \\
\hline
\end{tabular}

Figure S75. HPLC chromatograms for, (a) rac-6; (b) (P)-6; (c) (M)-6. 
6. For Helicenoid di-aldehyde, 7:

Column

Mobile Phase

Flow rate

Detector

Temperature
: Chiralcel® IC [4.6 mm i.d. x $250 \mathrm{~mm}$ ]

: Hexane: DCM 4060

: $0.7 \mathrm{ml} / \mathrm{min}$

: UV $(\lambda=318 \mathrm{~nm})$

: $20^{\circ} \mathrm{C}$

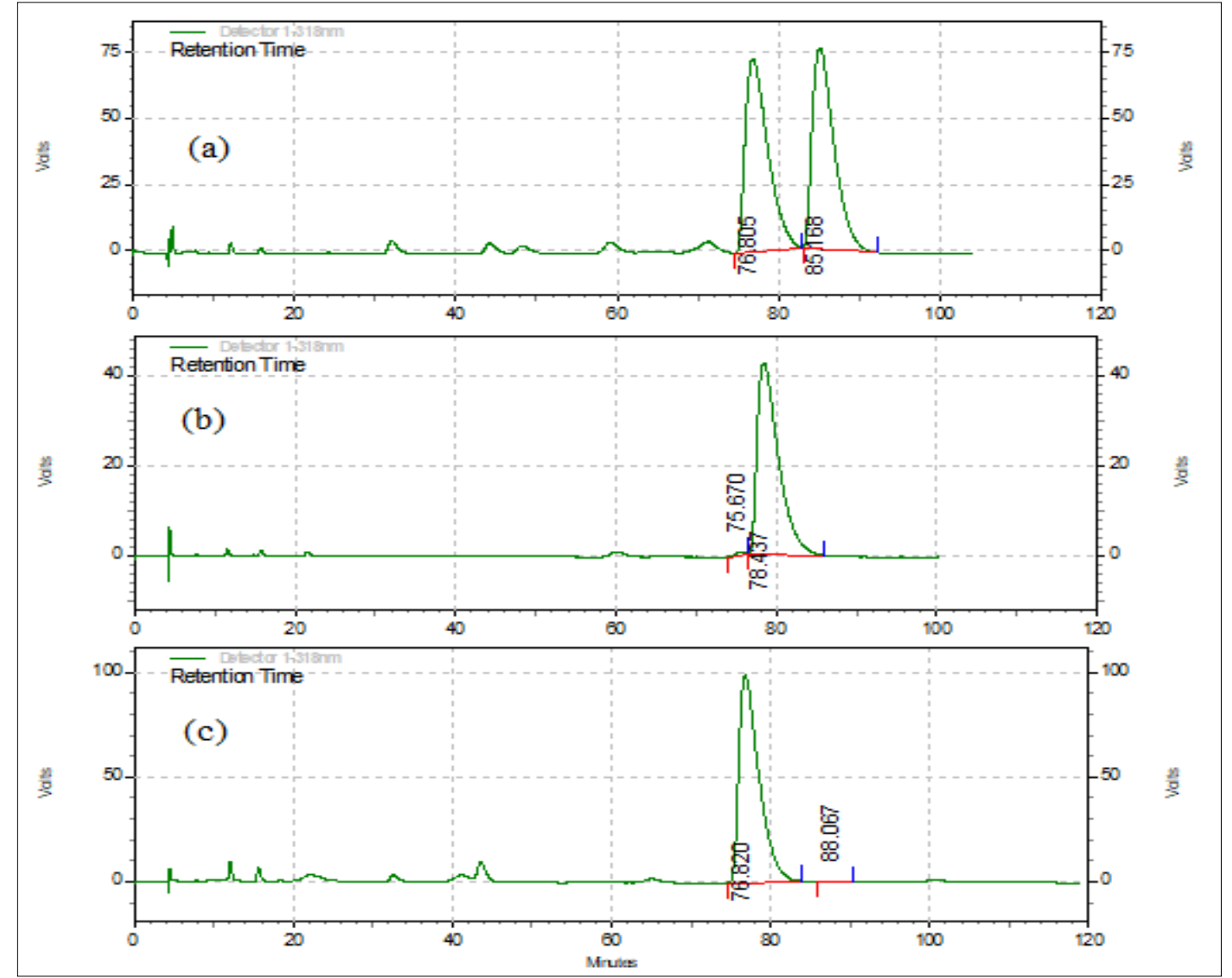

\begin{tabular}{|c|c|c|c|c|c|}
\hline \multicolumn{2}{|c|}{$r a c-7$} & \multicolumn{2}{c|}{$(P)-7$} & \multicolumn{2}{c|}{$(M)-7$} \\
\hline $\begin{array}{c}\text { Retention } \\
\text { Time }\end{array}$ & Area \% & $\begin{array}{c}\text { Retention } \\
\text { Time }\end{array}$ & Area \% & $\begin{array}{c}\text { Retention } \\
\text { Time }\end{array}$ & Area \% \\
\hline 76.80 & 49.96 & 75.67 & 0.65 & 76.82 & 99.81 \\
\hline 85.16 & 50.04 & 78.43 & 99.35 & 88.06 & 0.19 \\
\hline
\end{tabular}

Figure S76. HPLC chromatograms for, (a) rac-7; (b) (P)-7; (c) (M)-7. 
7. For helicenoid diol, 9:

Column

Mobile Phase

Flow rate

Detector

Temperature
: Chiralcel® IA [4.6 mm i.d. x $250 \mathrm{~mm}$ ]

: Hexane: IPA (80:20)

: $0.7 \mathrm{ml} / \mathrm{min}$

: $\mathrm{UV}(\lambda=315 \mathrm{~nm})$

: $20^{\circ} \mathrm{C}$

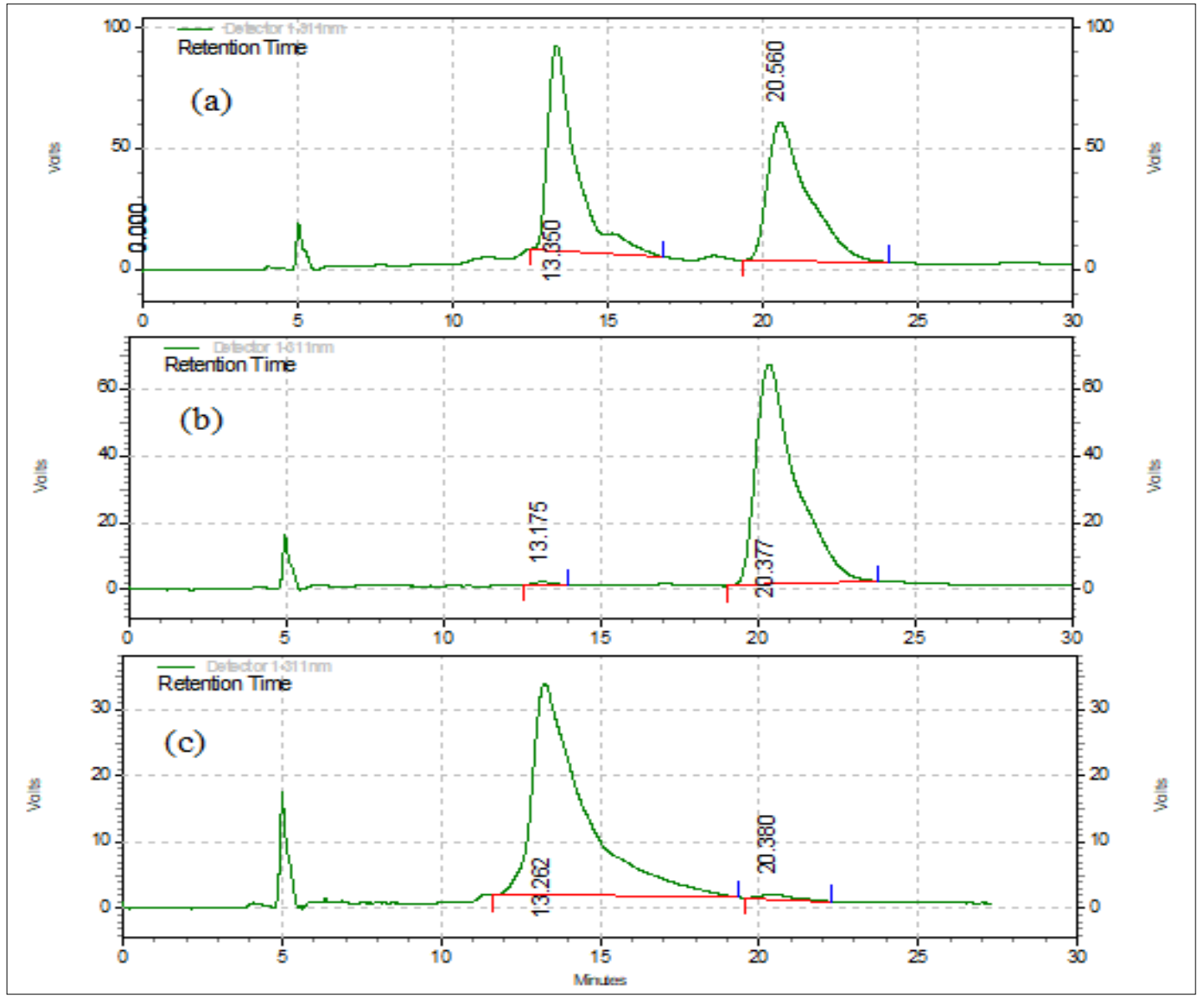

\begin{tabular}{|c|c|c|c|c|c|}
\hline \multicolumn{2}{|c|}{$r a c-9$} & \multicolumn{2}{c|}{$(P)-9$} & \multicolumn{2}{c|}{$(M)$-9 } \\
\hline $\begin{array}{c}\text { Retention } \\
\text { Time }\end{array}$ & Area \% & $\begin{array}{c}\text { Retention } \\
\text { Time }\end{array}$ & Area \% & $\begin{array}{c}\text { Retention } \\
\text { Time }\end{array}$ & Area \% \\
\hline 13.35 & 50.94 & 13.17 & 0.68 & 13.26 & 98.70 \\
\hline 20.56 & 49.06 & 20.37 & 99.32 & 20.38 & 1.30 \\
\hline
\end{tabular}

Figure S77. HPLC chromatograms for, (a) rac-9; (b) (P)-9; (c) (M)-9. 
8. For helicenoid mono aldehyde, 11:

Column

Mobile Phase

Flow rate

Detector

Temperature
: Chiralcel® IC [4.6 mm i.d. x $250 \mathrm{~mm}$ ]

: Hexane: DCM (80:20)

: $0.7 \mathrm{ml} / \mathrm{min}$

: $\mathrm{UV}(\lambda=314 \mathrm{~nm})$

: $20^{\circ} \mathrm{C}$

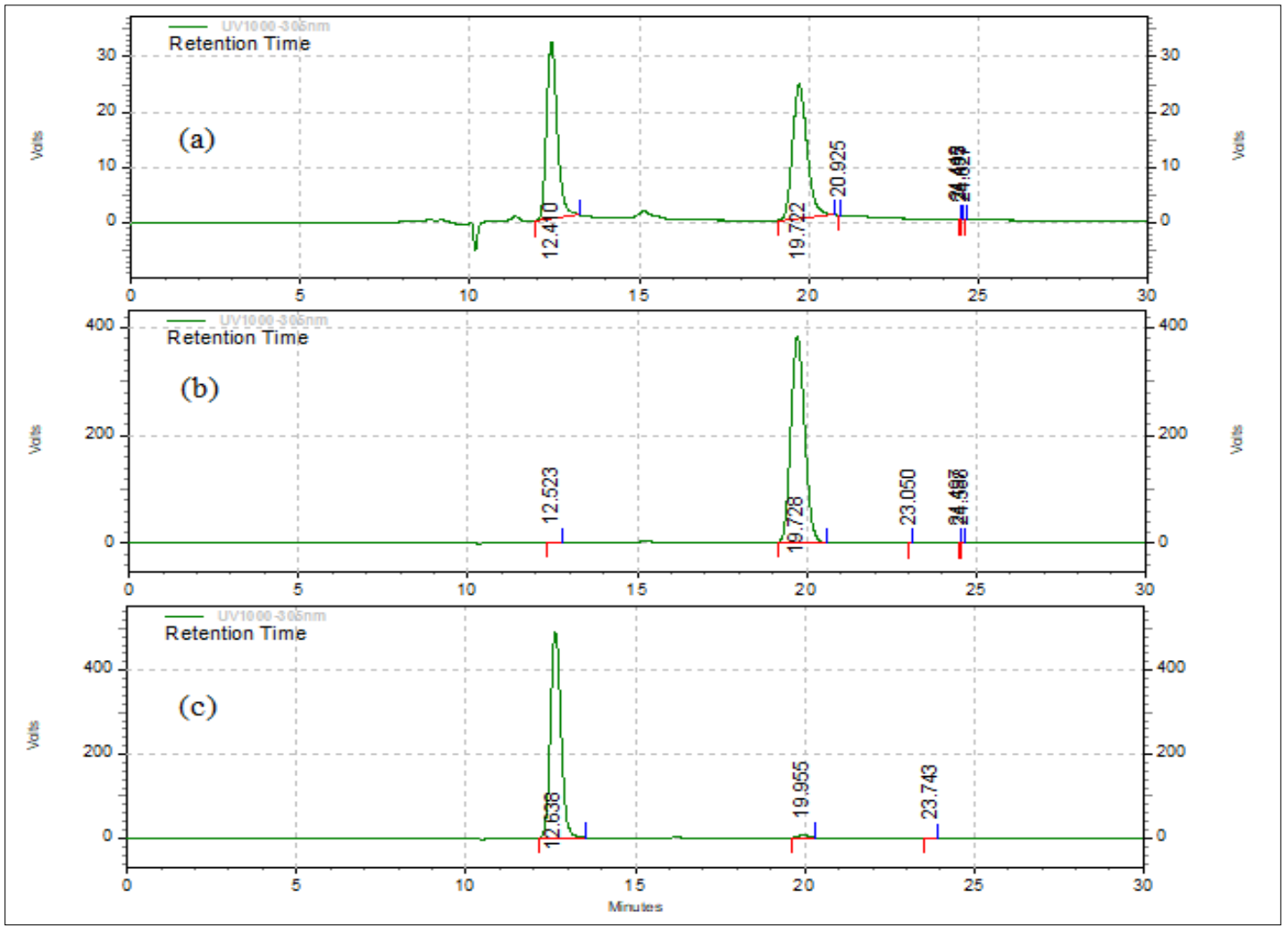

\begin{tabular}{|c|c|c|c|c|c|}
\hline \multicolumn{2}{|c|}{$r a c-11$} & \multicolumn{2}{|c|}{$(P)-11$} & \multicolumn{2}{c|}{$(M)-11$} \\
\hline $\begin{array}{c}\text { Retention } \\
\text { Time }\end{array}$ & Area \% & $\begin{array}{c}\text { Retention } \\
\text { Time }\end{array}$ & Area \% & $\begin{array}{c}\text { Retention } \\
\text { Time }\end{array}$ & Area \% \\
\hline 12.410 & 50.91 & 12.52 & 0.26 & 12.63 & 98.88 \\
\hline 19.722 & 50.50 & 19.728 & 99.74 & 19.95 & 1.12 \\
\hline
\end{tabular}

Figure S78. HPLC chromatograms for, (a) rac-11; (b) (P)-11; (c) (M)-11. 
9. For helicenoid mono alocohol, 12:

Column

Mobile Phase

Flow rate

Detector

Temperature
: Chiralcel® IA [4.6 mm i.d. x $250 \mathrm{~mm}$ ]

: Hexane: IPA (90:10)

: $0.5 \mathrm{ml} / \mathrm{min}$

: $\mathrm{UV}(\lambda=314 \mathrm{~nm})$

: $20^{\circ} \mathrm{C}$

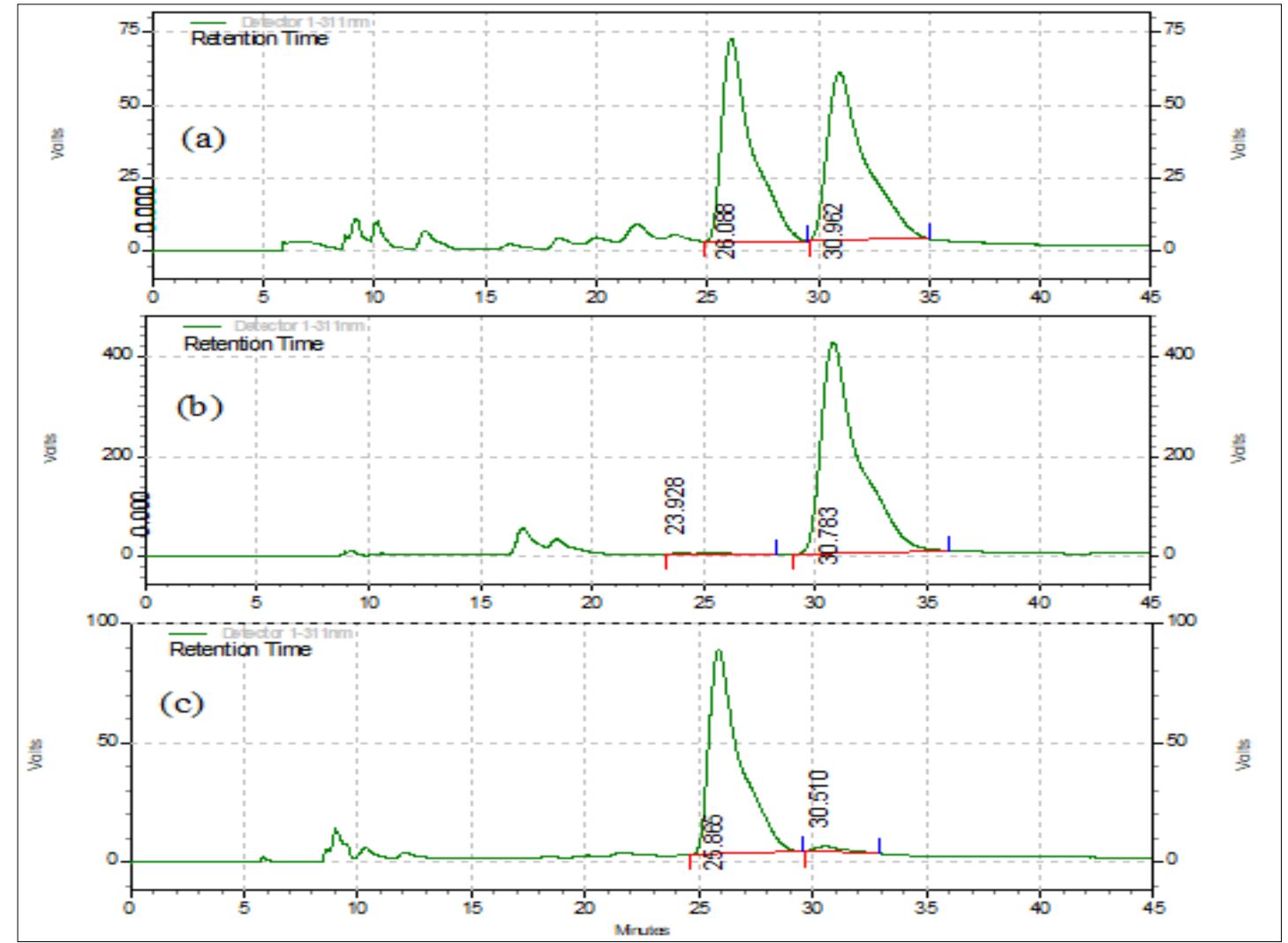

\begin{tabular}{|c|c|c|c|c|c|}
\hline \multicolumn{2}{|c|}{} & \multicolumn{2}{|c|}{$(P)-12$} & \multicolumn{2}{c|}{$(M)-12$} \\
\hline $\begin{array}{c}\text { Retention } \\
\text { Time }\end{array}$ & Area \% & $\begin{array}{c}\text { Retention } \\
\text { Time }\end{array}$ & Area \% & $\begin{array}{c}\text { Retention } \\
\text { Time }\end{array}$ & Area \% \\
\hline 26.08 & 48.99 & 23.92 & 0.64 & 25.86 & 97.22 \\
\hline 30.96 & 51.01 & 30.78 & 99.36 & 30.51 & 2.78 \\
\hline
\end{tabular}

Figure S79. HPLC chromatograms for, (a) rac-12; (b) (P)-12; (c) (M)-12. 


\section{Chiroptical studies:}

UV-Vis absorption and Circular dischroisom (CD) spectra for helicenoid(s) enantiomers:

Method: All samples were measured in acetonitrile solvent at $5 \times 10^{-6} \mathrm{M}$ concentration.

1. Furo Fused BINOL, 1:

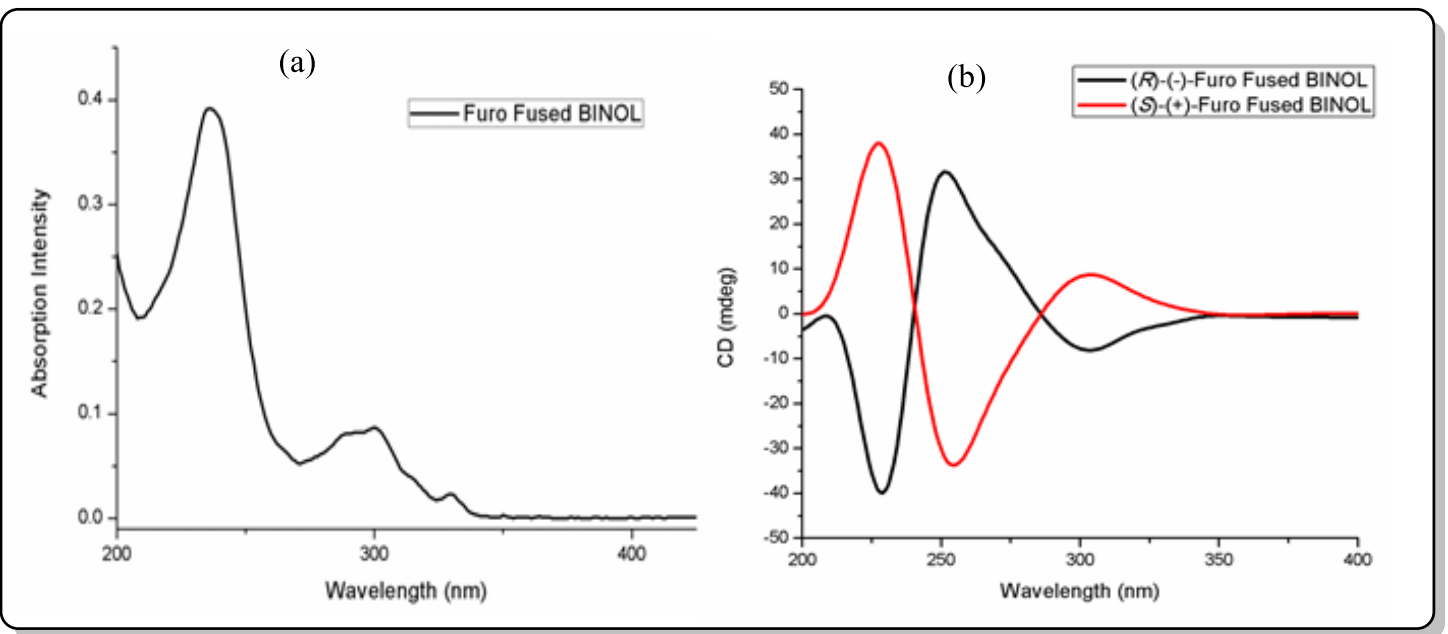

Figure S80. (a) UV spectrum of furo fused BINOL, 1; (b) CD spectrum of furo fused BINOL, 1.

\section{Helicenoid, 2:}
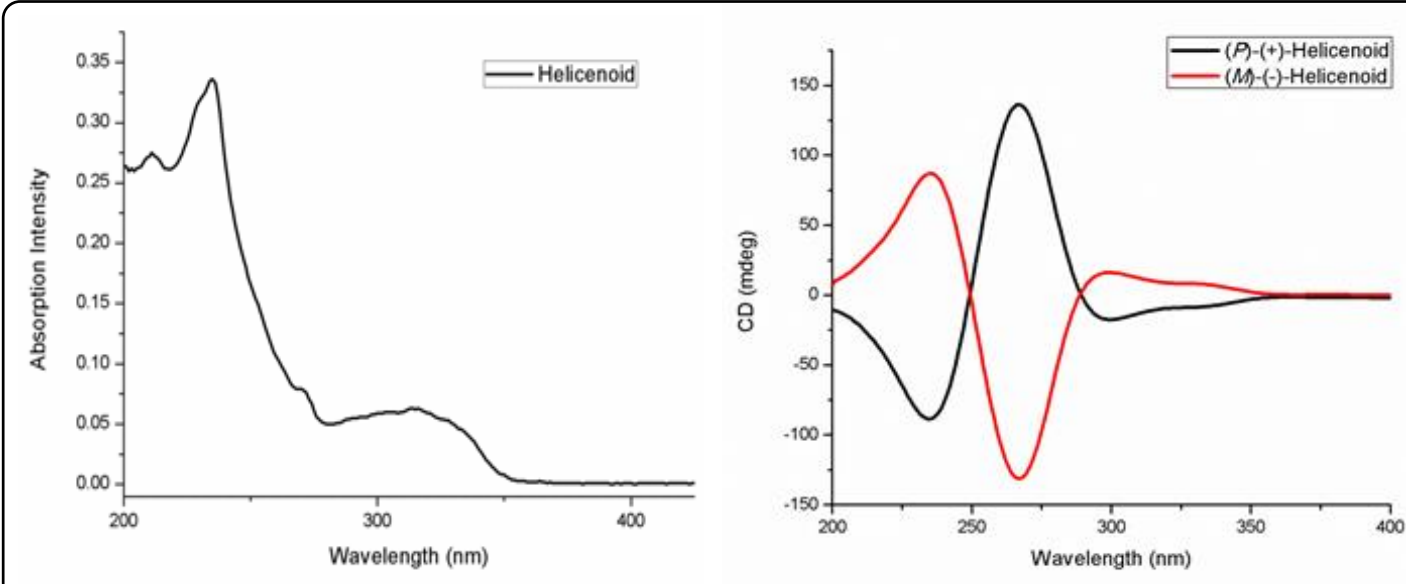

Figure S81: (a) UV spectrum of helicenoid, 2; (b) CD spectrum of helicenoid, 2. 


\section{Helicenoid dibromide, 3:}

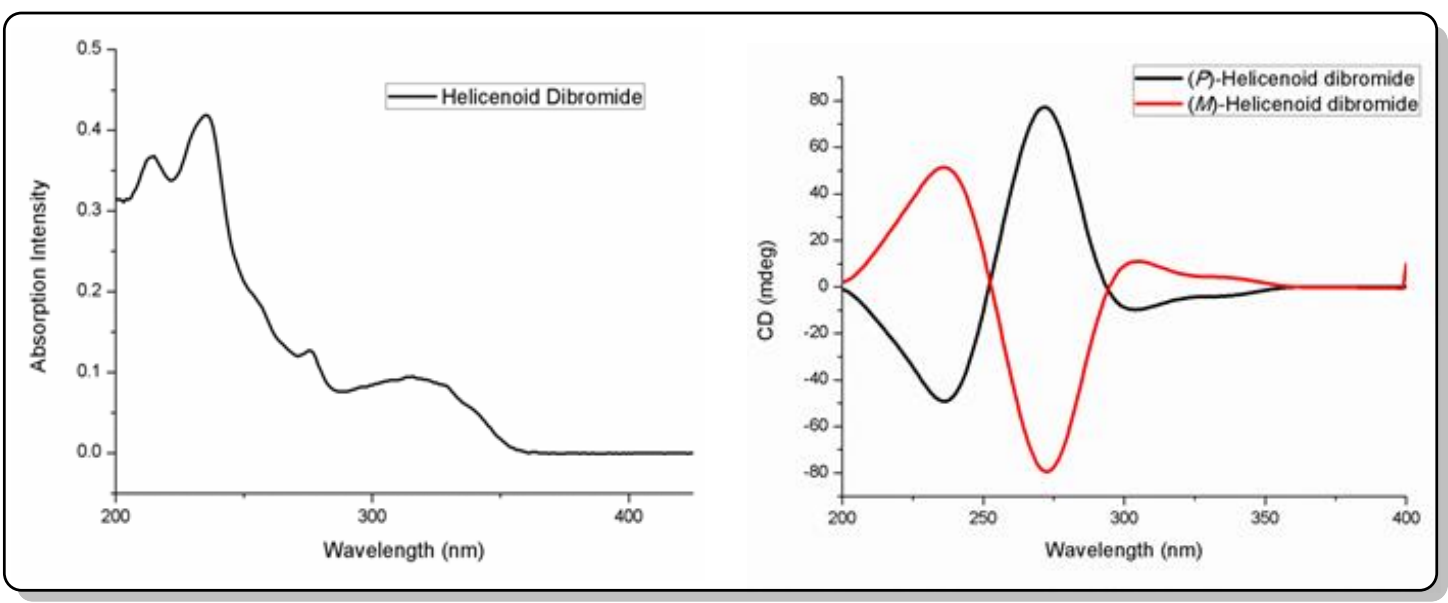

Figure S82. (a) UV spectrum of helicenoid dibromide, 3; (b) CD spectrum of helicenoid dibromide, 3.

\section{Helicenoid mono bromo acid, 5:}

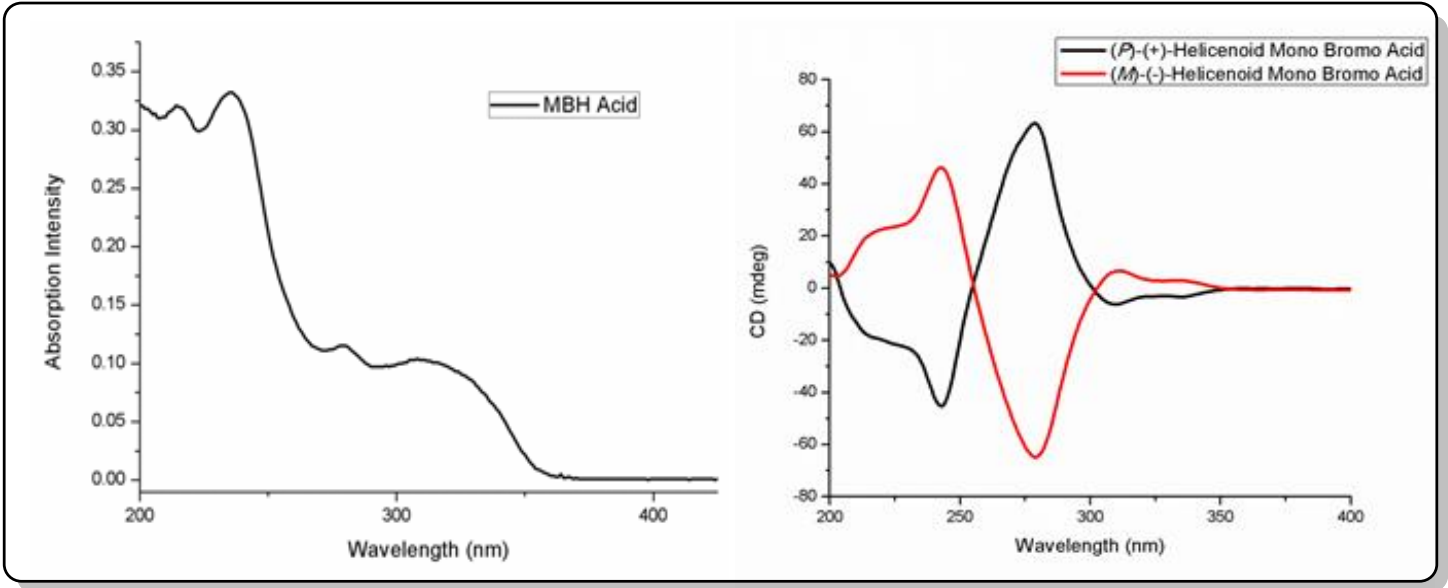

Figure S83: (a) UV spectrum of helicenoid mono bromo acid, 5; (b) CD spectrum of helicenoid mono bromo acid, 5. 


\section{2,16-distyryl helicenoid, 6:}
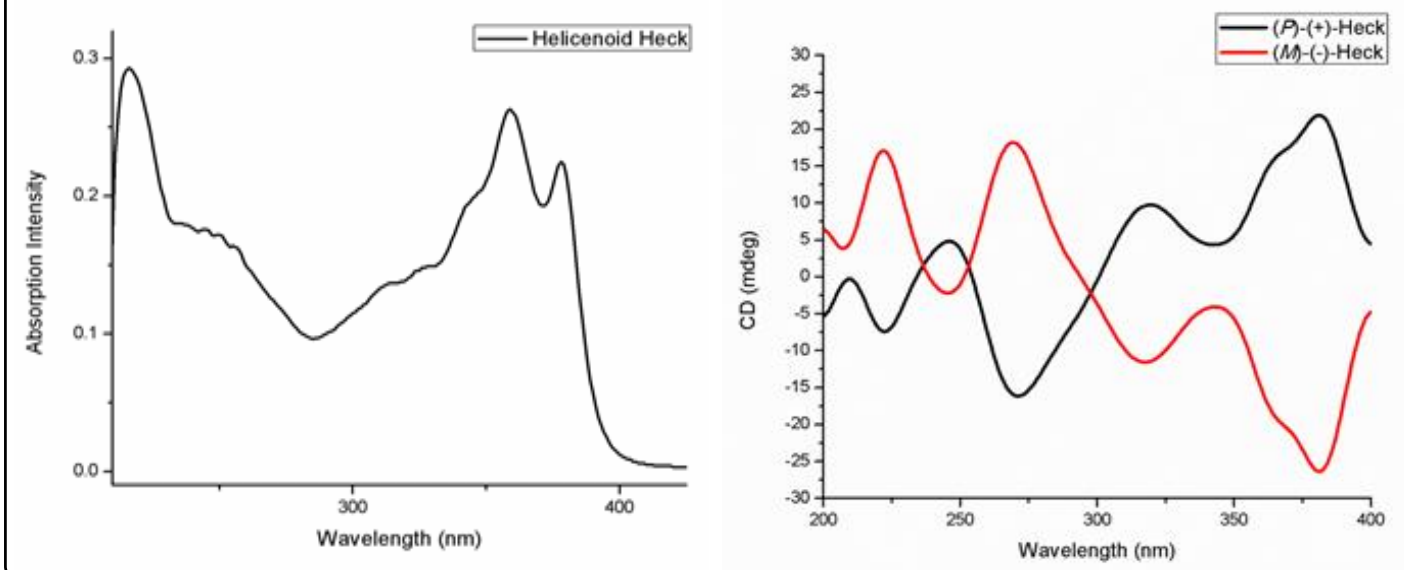

Figure S84: (a) UV spectrum of 2,2'-distyryl helicenoid, 6; (b) CD spectrum of styrene incorporated helicenoid, 6.

\section{Helicenoid dialdehyde, 7:}

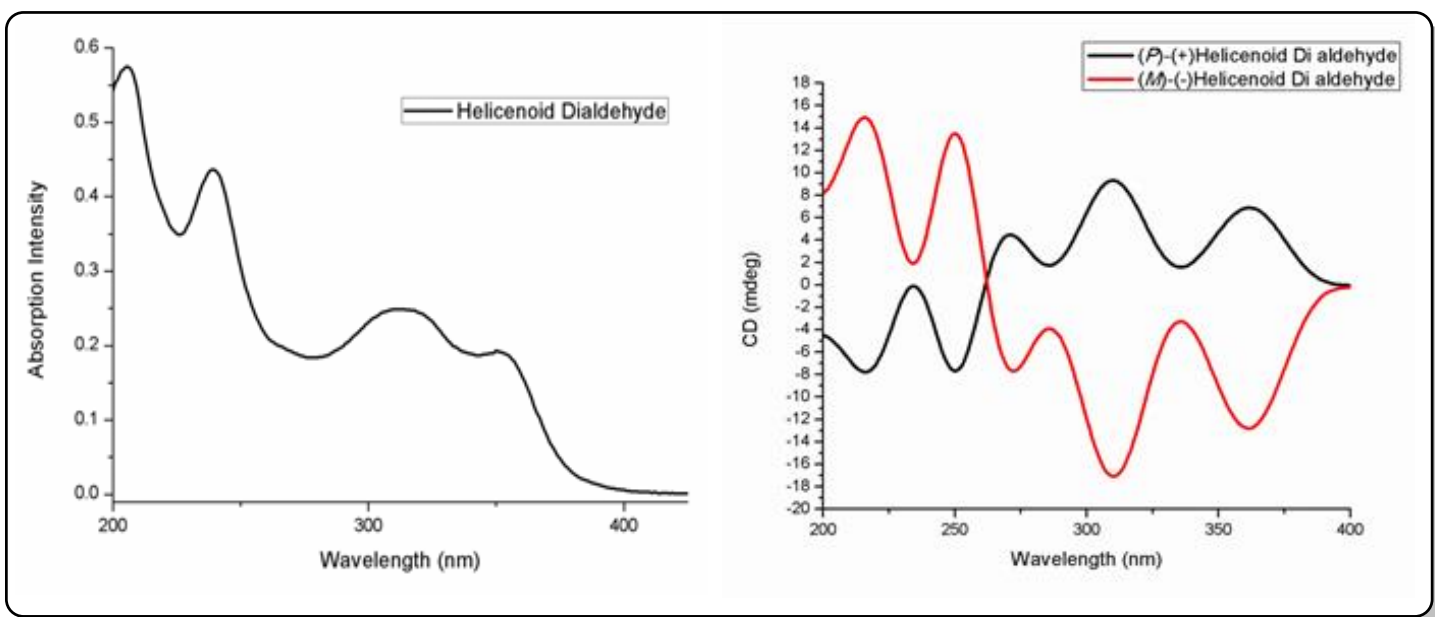

Figure S85: (a) UV spectrum of helicenoid dialdehyde, 7; (b) CD spectrum of helicenoid dialdehyde, 7. 
7. Helicenoid Diacid, 8:

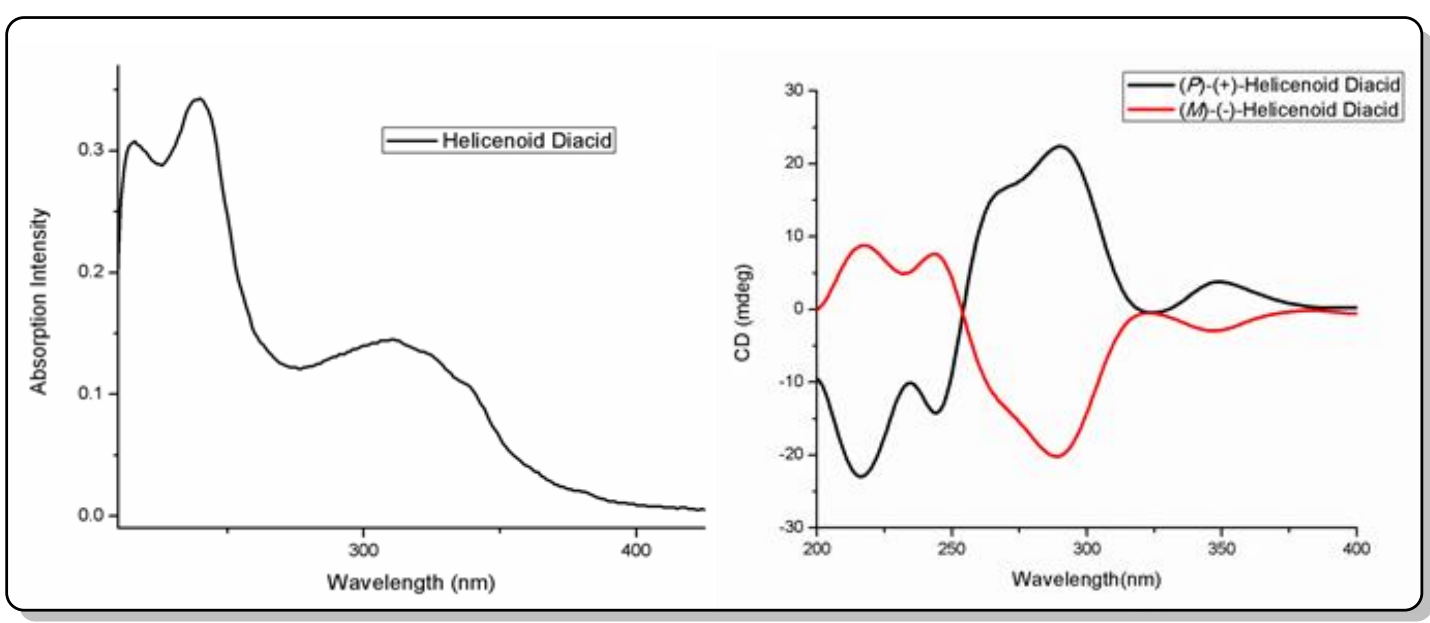

Figure S86: (a) UV spectrum of helicenoid diacid, 8; (b) CD spectrum of helicenoid diacid, 8.

\section{Helicenoid diol, 9:}

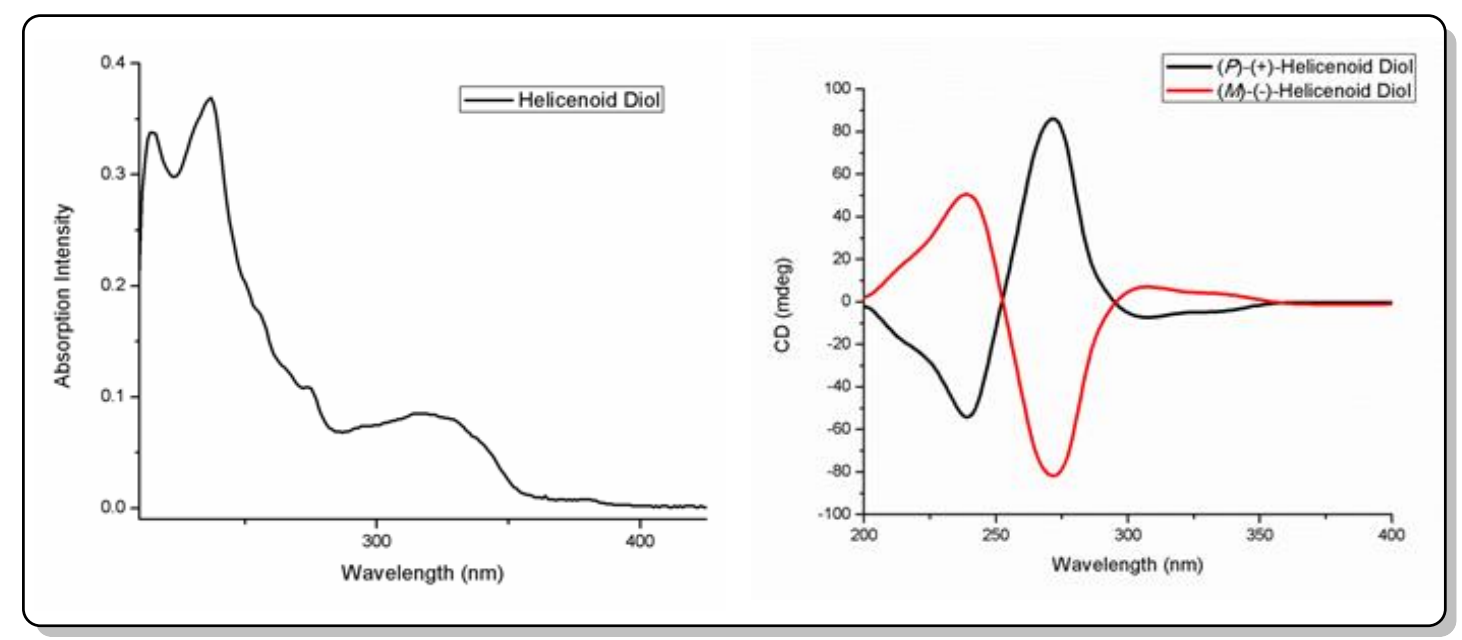

Figure S87: (a) UV spectrum of helicenoid diol, 9; (b) CD spectrum of helicenoid diol, 9. 


\section{Helicenoid mono aldehyde, 11:}

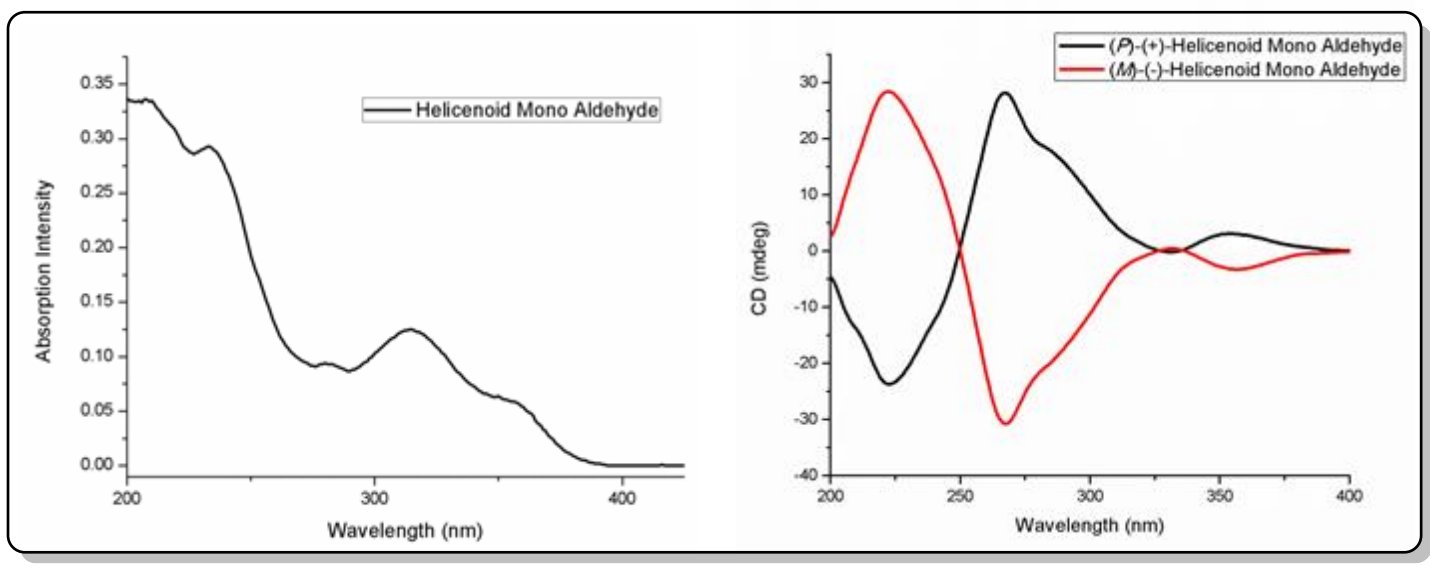

Figure S88: (a) UV spectrum of helicenoid mono aldehyde, 11; (b) CD spectrum of helicenoid diol mono aldehyde, 11.

\section{Helicenoid mono alcohol, 12:}
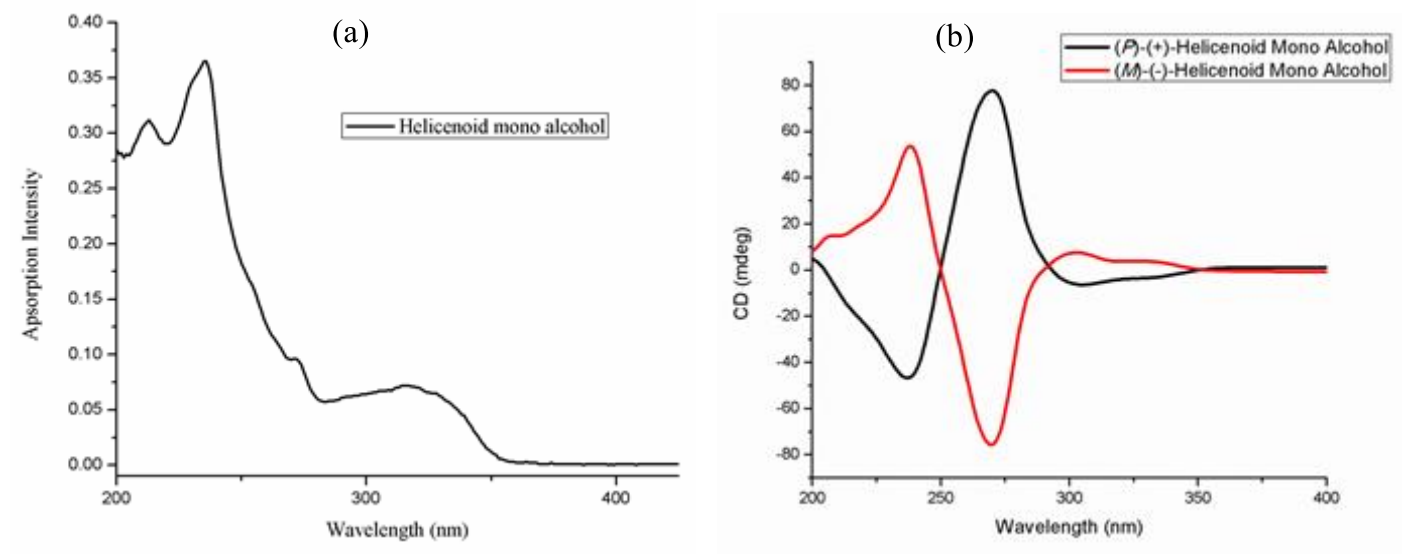

Figure S89: (a) UV spectrum of helicenoid mono alcohol, 12; (b) CD spectrum of helicenoid diol mono alcohol, 12.

\subsection{Kuhn's Factor / Anisotropy factor ' $g$ ' Value calculations:}

Calculations were performed at $2^{\text {nd }}$ Cotton effect (CE) wavelength. Abs. and $\Theta$ represents the UV-Vis absorbance and CD amplitude value at $2^{\text {nd }} \mathrm{CE}$ wavelength, respectively. The $g$ factor amplitude $(\Theta)$ is the total amplitude of the $g$ factor in the region corresponding to the Cotton effects in the CD spectra. The accuracy of the $g$ factor amplitude values is $\left( \pm 2 \times 10^{-3}\right)$ as determined from baseline evaluation.

Concentration $=5 \times 10^{-6} \mathrm{M}$

Formulae used: 
1. $\Delta \boldsymbol{\varepsilon}=\mathbf{\Theta} / \mathbf{3 2 9 8 0} \boldsymbol{c l}($ for $C D) \quad 2 . \boldsymbol{\varepsilon}=\mathbf{A} / \mathrm{cl}($ for $U v-$ Vis $) \quad 3 . g=\Delta \boldsymbol{\varepsilon} / \boldsymbol{\varepsilon}$

Where,

$\boldsymbol{\Theta}=$ sample's ellipticity measured in millidegrees (mdeg)

$\mathbf{c}=$ sample's concentration

$l=$ path length $(\mathrm{cm})$

$\Delta \varepsilon=$ molar ellipticity $\left(\Delta \varepsilon=\varepsilon_{1}-\varepsilon_{\mathrm{r}}\right)$

$\mathbf{A}=$ absorption in UV-vis spectroscopy

Table S2: Kuhn's Factor / Anisotropy factor 'g' Value

\begin{tabular}{|c|c|c|c|c|c|}
\hline $\begin{array}{l}\text { Helicenoid Compound } \\
\qquad(\mathbf{7 9 - 9 0 )}\end{array}$ & $\begin{array}{c}\text { Abs. } \\
\text { (UV-Vis) }\end{array}$ & $\begin{array}{r}\Theta \\
\text { mdeg } \\
\text { at } \\
2^{\text {nd }} \mathrm{CE}\end{array}$ & $\begin{array}{l}\Delta \varepsilon \times 10^{2} \\
\mathrm{~cm}^{-1} \mathrm{M}^{-1}\end{array}$ & $2^{\text {nd }} \mathrm{CE}$ & $\begin{array}{c}\mathrm{g}=\Delta \varepsilon / \varepsilon * 10^{-} \\
3 \\
\left(\text { at } 2^{\text {nd }} \mathrm{CE}\right)\end{array}$ \\
\hline $\begin{array}{c}(R) \text {-Furo Fused } \\
\text { BINOL, } 1\end{array}$ & 0.182 & 31.65 & 1.919 & + & 5.271 \\
\hline$(P)$-Helicenoid, 2 & 0.081 & 136.51 & 8.278 & + & 51.1 \\
\hline $\begin{array}{c}(P) \text {-Helicenoid } \\
\text { dibromide, } 3\end{array}$ & 0.121 & 77.27 & 4.685 & + & 19.36 \\
\hline $\begin{array}{l}(P) \text {-Mono bromo } \\
\text { helicenoid acid, } \mathbf{5}\end{array}$ & 0.115 & 63.31 & 3.833 & + & 16.67 \\
\hline $\begin{array}{c}(P) \text {-Helicenoid } \\
\text { incorporated styrene, } 6\end{array}$ & 0.203 & $\begin{array}{c}21.91 \\
\left(1^{\text {st }} \mathrm{CE}\right)\end{array}$ & 1.32 & $\begin{array}{c}+ \\
\left(1^{\mathrm{st}} \mathrm{CE}\right)\end{array}$ & 3.25 \\
\hline $\begin{array}{l}\text { (M)-Helicenoid } \\
\text { dialdehyde, } 7\end{array}$ & 0.249 & 17.09 & 1.036 & - & 2.08 \\
\hline$(P)$-Helicenoid diol, 9 & 0.108 & 86.15 & 5.22 & + & 24.17 \\
\hline $\begin{array}{c}(P) \text {-Helicenoid di-acid, } \\
\mathbf{8}\end{array}$ & 0.131 & 22.39 & 1.35 & + & 5.15 \\
\hline $\begin{array}{c}(P) \text {-Helicenoid mono } \\
\text { aldehyde, } \mathbf{1 1}\end{array}$ & 0.103 & 28.25 & 1.71 & + & 8.30 \\
\hline $\begin{array}{c}(P) \text {-Helicenoid mono } \\
\text { alcohol, } 12\end{array}$ & 0.095 & 77.83 & 4.71 & + & 27.79 \\
\hline
\end{tabular}

Calculations for Kuhn's Factor / Anisotropy factor $(\mathrm{g}) ;\left(\mathrm{c}=5 \times 10^{-6} \mathrm{M}\right.$ in Acetonitrile) (R)-Furo Fused BINOL; CD $2^{\text {nd }} \mathrm{CE}$ at $251 \mathrm{~nm}$ :

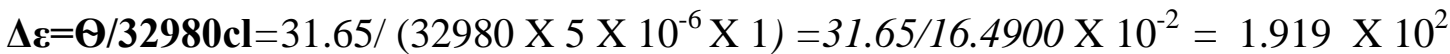

$\boldsymbol{\varepsilon}=\mathbf{A} / \mathbf{c l}=0.182 / 5 \times 10^{-6} \mathrm{X} 1=0.0364 \times 10^{6}$ 
$\mathrm{g}=\Delta \varepsilon / \varepsilon=1.919 \times 10^{2} / 0.0364 \times 10^{6}=\mathbf{5 . 2 7 1} \times \mathbf{1 0}^{-3}$

(P)-Helicenoid; CD $2^{\text {nd }} \mathrm{CE}$ at $267 \mathrm{~nm}$ :

$\Delta \boldsymbol{\varepsilon}=\boldsymbol{\Theta} / \mathbf{3 2 9 8 0} \mathrm{cl}=136.51 /\left(32980 \times 5 \times 10^{-6}\right.$ X 1$)=136.51 / 16.4900 \times 10^{-2}=8.278 \times 10^{2}$ $\boldsymbol{\varepsilon}=\mathbf{A} / \mathbf{c l}=0.081 / 5 \times 10^{-6} \mathrm{X} 1=0.0162 \times 10^{6}$

$\mathrm{g}=\Delta \varepsilon / \varepsilon=8.278 \times 10^{2} / 0.0162 \times 10^{6}=\mathbf{5 1 . 1} \times \mathbf{1 0}^{-3}$

(P)-Helicenoid Dibromide; CD $2^{\text {nd }} \mathrm{CE}$ at $272 \mathrm{~nm}$ :

$\Delta \boldsymbol{\varepsilon}=\boldsymbol{\Theta} / 32980 \mathrm{cl}=77.27 /\left(32980 \times 5 \times 10^{-6}\right.$ X 1$)=77.27 / 16.4900 \times 10^{-2}=4.685 \times 10^{2}$ $\boldsymbol{\varepsilon}=\mathbf{A} / \mathbf{c l}=0.121 / 5 \times 10^{-6} \mathrm{X} 1=0.0242 \times 10^{6}$ $\mathrm{g}=\Delta \varepsilon / \varepsilon=4.685 \times 10^{2} / 0.0242 \times 10^{6}=\mathbf{1 9 . 3 6} \times \mathbf{1 0}^{-3}$

(P)-Mono Bromo Helicenoid Acid; CD $2^{\text {nd }} \mathrm{CE}$ at 279 nm:

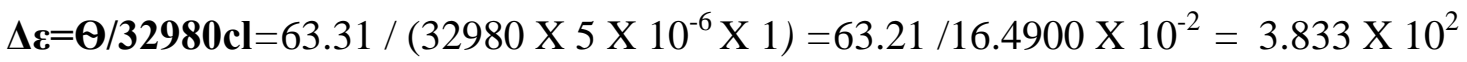
$\boldsymbol{\varepsilon}=\mathbf{A} / \mathbf{c l}=0.115 / 5 \times 10^{-6} \mathrm{X} 1=0.023 \times 10^{6}$ $\mathrm{g}=\Delta \varepsilon / \varepsilon=3.833 \times 10^{2} / 0.023 \times 10^{6}=\mathbf{1 6 . 6 7} \times \mathbf{1 0}^{-3}$

$(P)$-Helicenoid Heck; CD $1^{\text {st }} \mathrm{CE}$ at $381 \mathrm{~nm}$

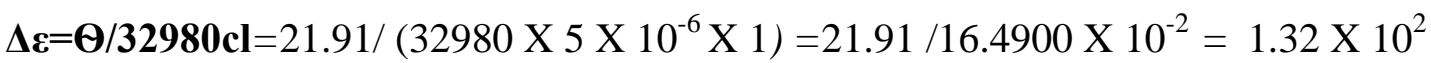
$\boldsymbol{\varepsilon}=\mathbf{A} / \mathbf{c l}=0.203 / 5 \times 10^{-6} \mathrm{X} 1=0.0406 \times 10^{6}$ $\mathrm{g}=\Delta \varepsilon / \varepsilon=1.32 \times 10^{2} / 0.0406 \times 10^{6}=\mathbf{3 . 2 5} \times \mathbf{1 0}^{-3}$

(M)-Helicenoid Dialdehyde; CD $2^{\text {nd }} \mathrm{CE}$ at $310 \mathrm{~nm}$

$\Delta \boldsymbol{\varepsilon}=\boldsymbol{\Theta} / 32980 \mathrm{cl}=17.09 /\left(32980 \times 5 \times 10^{-6}\right.$ X 1$)=17.09 / 16.4900 \times 10^{-2}=1.036 \mathrm{X} 10^{2}$ $\boldsymbol{\varepsilon}=\mathbf{A} / \mathbf{c l}=0.249 / 5 \times 10^{-6} \mathrm{X} 1=0.0498 \times 10^{6}$ $\mathrm{g}=\Delta \varepsilon / \varepsilon=1.036 \times 10^{2} / 0.0498 \times 10^{6}=\mathbf{2 . 0 8 0} \times \mathbf{1 0}^{-3}$

$(P)-H e l i c e n o i d ~ D i o l ; C D 2^{\text {nd }} \mathrm{CE}$ at $272 \mathrm{~nm}$ $\Delta \boldsymbol{\varepsilon}=\boldsymbol{\Theta} / \mathbf{3 2 9 8 0} \mathrm{cl}=86.15 /\left(32980 \times 5 \times 10^{-6} \mathrm{X} 1\right)=86.15 / 16.4900 \times 10^{-2}=5.22 \times 10^{2}$ $\boldsymbol{\varepsilon}=\mathbf{A} / \mathbf{c l}=0.108 / 5 \times 10^{-6} \mathrm{X} 1=0.0216 \times 10^{6}$ $\mathrm{g}=\Delta \varepsilon / \varepsilon=5.22 \times 10^{2} / 0.0216 \times 10^{6}=\mathbf{2 4 . 1 7} \times \mathbf{1 0}^{-3}$

$(P)$-Helicenoid Diacid; CD $2^{\text {nd }} C E$ at $290 \mathrm{~nm}$ $\Delta \boldsymbol{\varepsilon}=\boldsymbol{\Theta} / \mathbf{3 2 9 8 0} \mathrm{cl}=22.39 /\left(32980 \mathrm{X} 5 \mathrm{X} 10^{-6} \mathrm{X} 1\right)=22.39 / 16.4900 \times 10^{-2}=1.35 \mathrm{X} 10^{2}$ $\boldsymbol{\varepsilon}=\mathbf{A} / \mathbf{c l}=0.131 / 5 \times 10^{-6} \mathrm{X} 1=0.0262 \times 10^{6}$ $\mathrm{g}=\Delta \varepsilon / \varepsilon=1.35 \times 10^{2} / 0.0262 \times 10^{6}=\mathbf{5 . 1 5} \times \mathbf{1 0}^{-3}$ (P)-Helicenoid Mono Aldehyde; CD $2^{\text {nd }} \mathrm{CE}$ at $267 \mathrm{~nm}$ $\Delta \boldsymbol{\varepsilon}=\boldsymbol{\Theta} / \mathbf{3 2 9 8 0} \mathrm{cl}=28.25 /\left(32980 \mathrm{X} 5 \mathrm{X} 10^{-6}\right.$ X 1$)=28.25 / 16.4900 \times 10^{-2}=1.71 \times 10^{2}$ $\boldsymbol{\varepsilon}=\mathbf{A} / \mathbf{c l}=0.103 / 5 \times 10^{-6} \mathrm{X} 1=0.0206 \times 10^{6}$ $\mathrm{g}=\Delta \varepsilon / \varepsilon=1.71 \times 10^{2} / 0.0206 \times 10^{6}=\mathbf{8 . 3 0} \times \mathbf{1 0}^{-3}$

(P)-Helicenoid Mono-alcohol; CD $2^{\text {nd }} \mathrm{CE}$ at $270 \mathrm{~nm}$ 


$$
\begin{aligned}
& \Delta \boldsymbol{\varepsilon}=\boldsymbol{\Theta} / \mathbf{3 2 9 8 0} \mathrm{cl}=77.83 /\left(32980 \times 5 \times 10^{-6} \times 1\right)=77.83 / 16.4900 \times 10^{-2}=4.71 \times 10^{2} \\
& \boldsymbol{\varepsilon}=\mathbf{A} / \mathbf{c l}=0.095 / 5 \times 10^{-6} \times 1=0.019 \times 10^{6} \\
& \mathrm{~g}=\Delta \varepsilon / \varepsilon=4.71 \times 10^{2} / 0.019 \times 10^{6}=\mathbf{2 4 . 7 9} \times \mathbf{1 0}^{-3}
\end{aligned}
$$

\section{Fluorescence Spectra of helicenoids:}

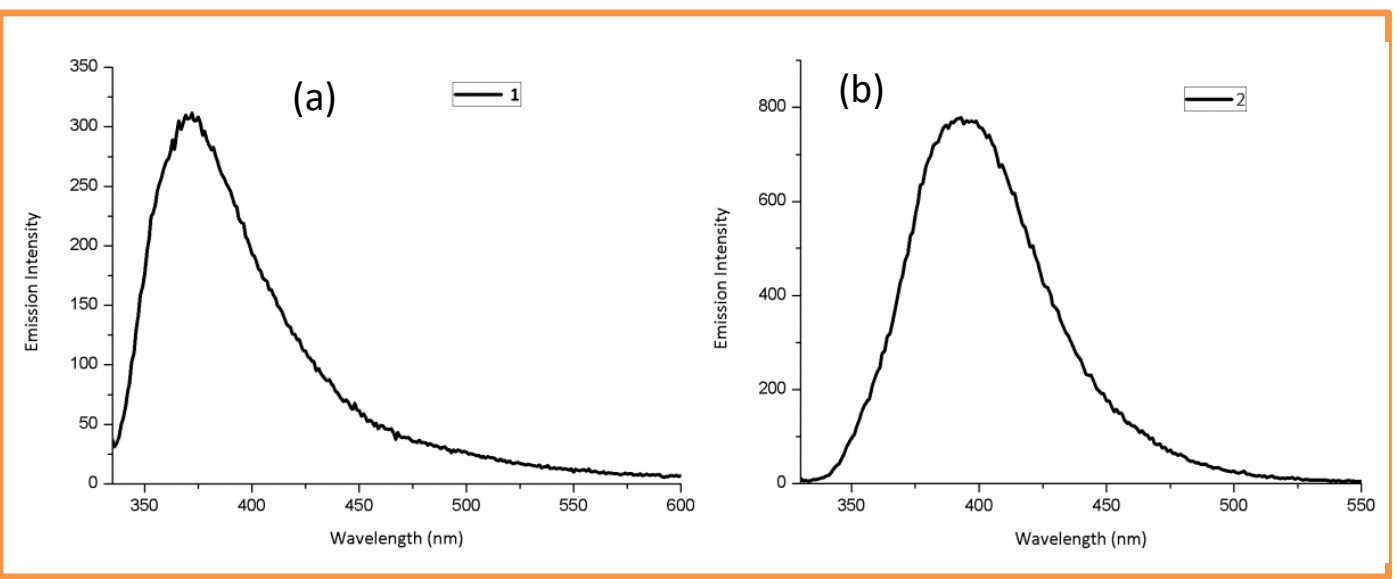

Figure S90. Fluorescence spectra of (a) Furo Fused BINOL, 1; (b) Helicenoid, 2
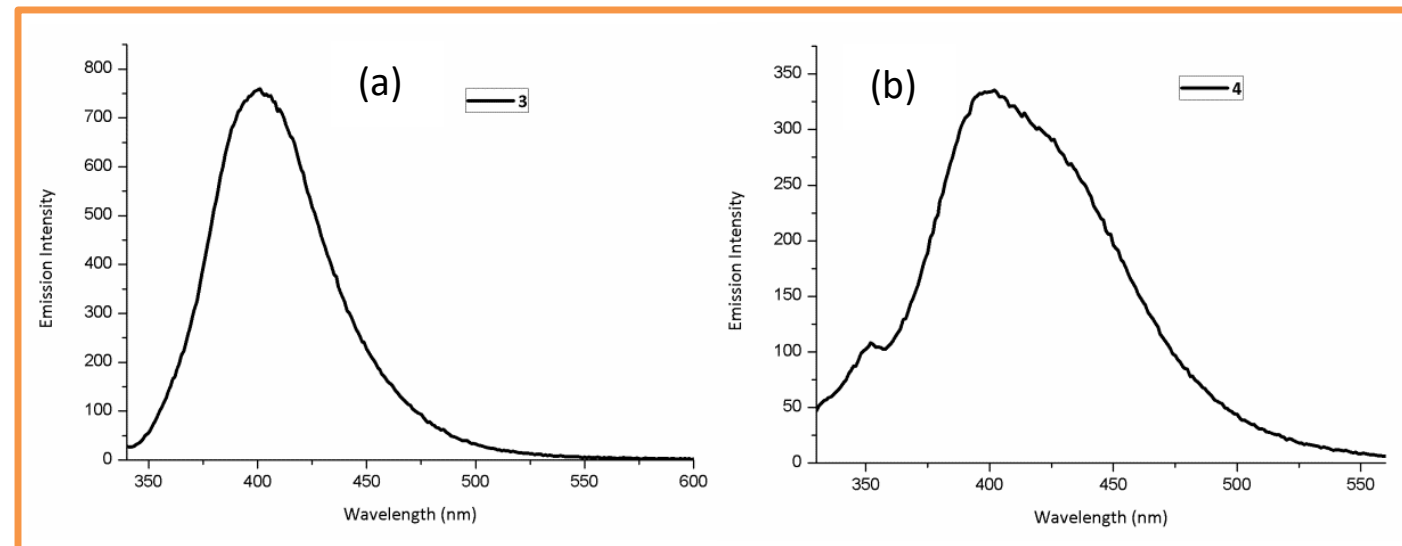

Figure S91. Fluorescence spectra of (a) Helicenoid dibromide, 3; (b) Helicenoid mono bromo cyanide, 4
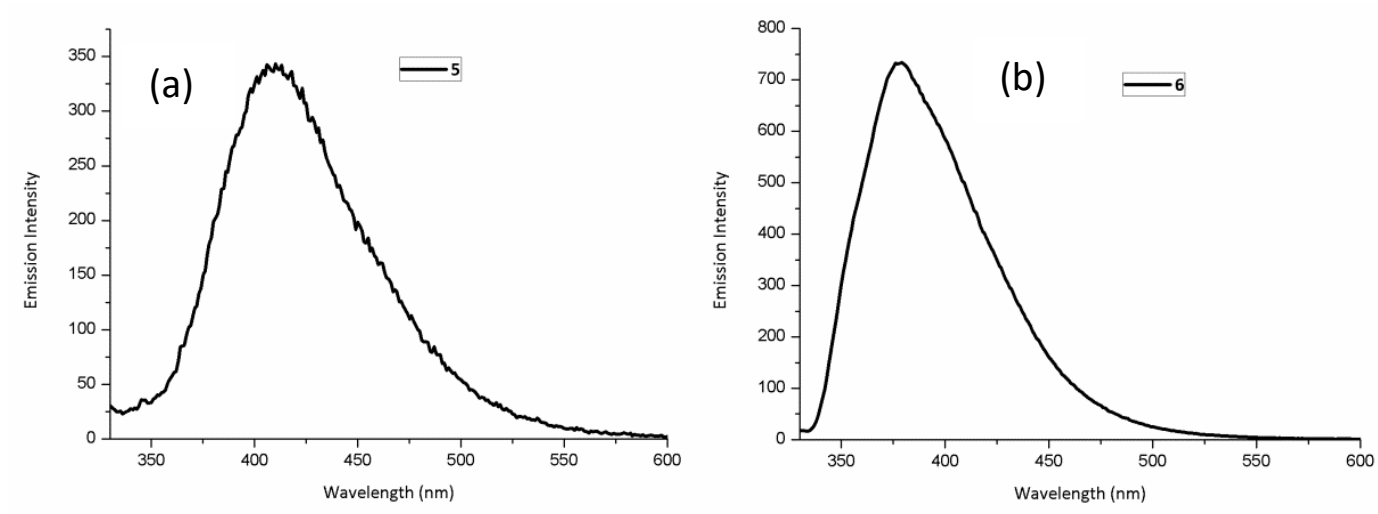

Figure S92. Fluorescence spectra of (a) Helicenoid mono bromo acid, 5; (b) 2,16 distyryl helicenoid, 6 


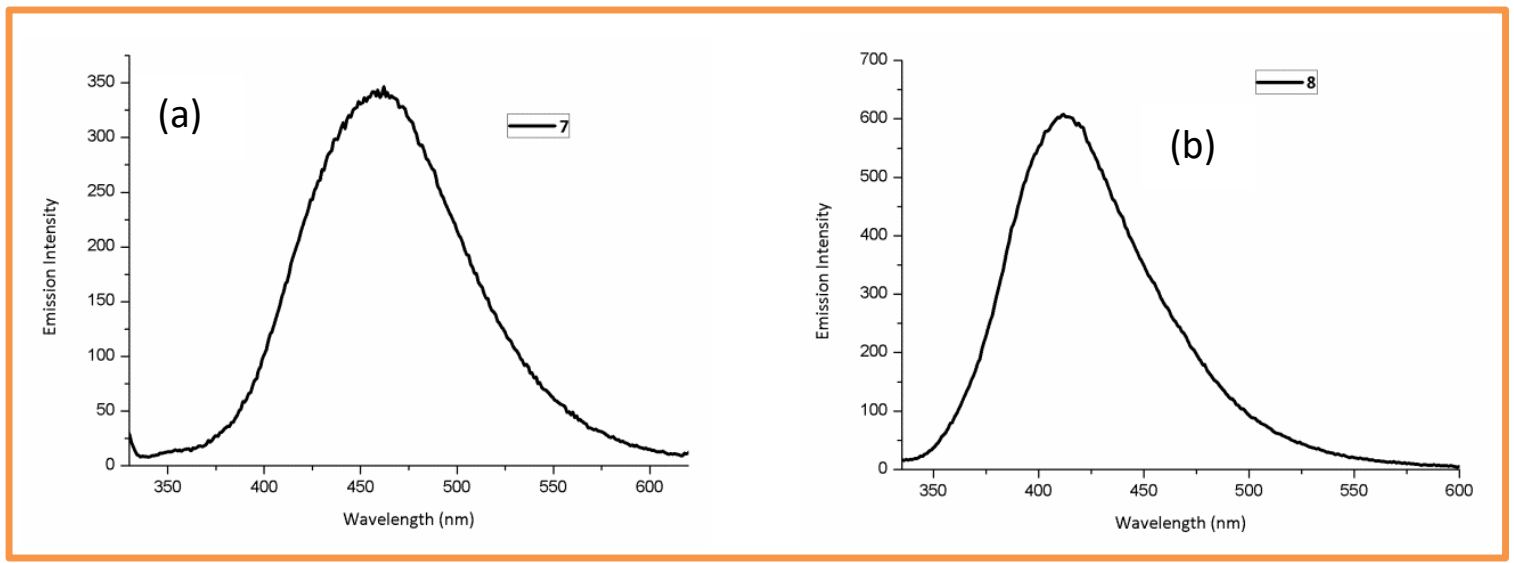

Figure S93. Fluorescence spectra of (a) Helicenoid dialdehyde, 7; (b) Helicenoid diacid, 8

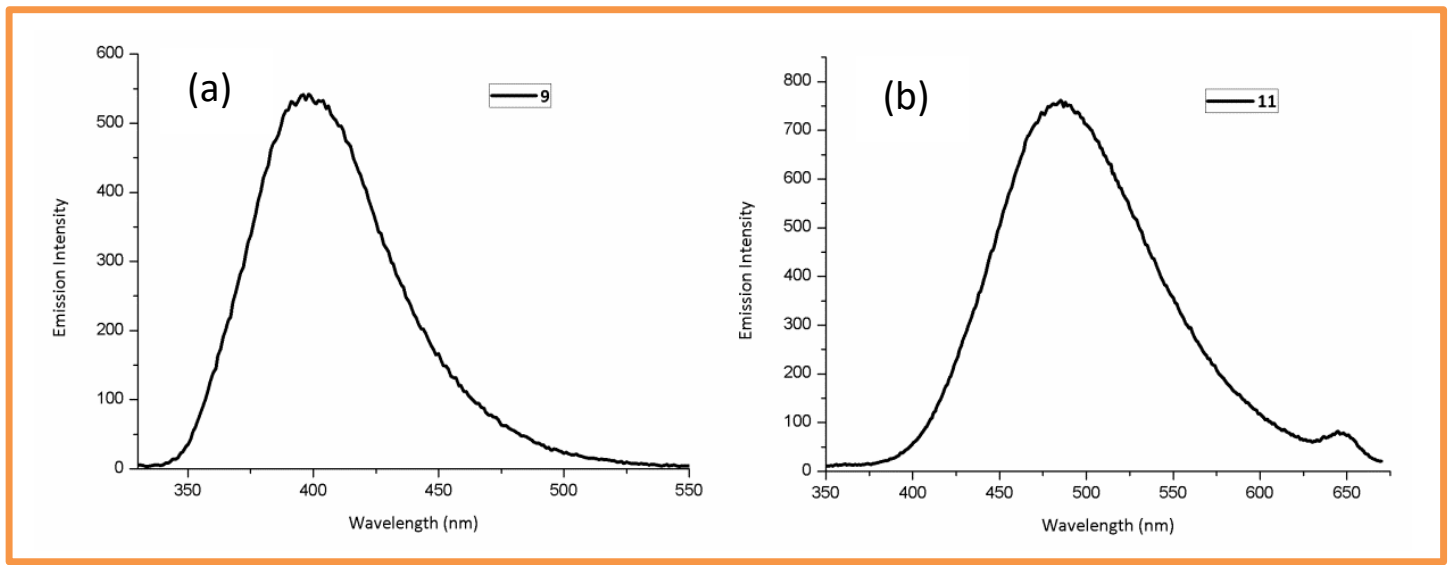

Figure S94. Fluorescence spectra of (a) Helicenoid diol, 9; (b) Helicenoid mono aldehyde, 11

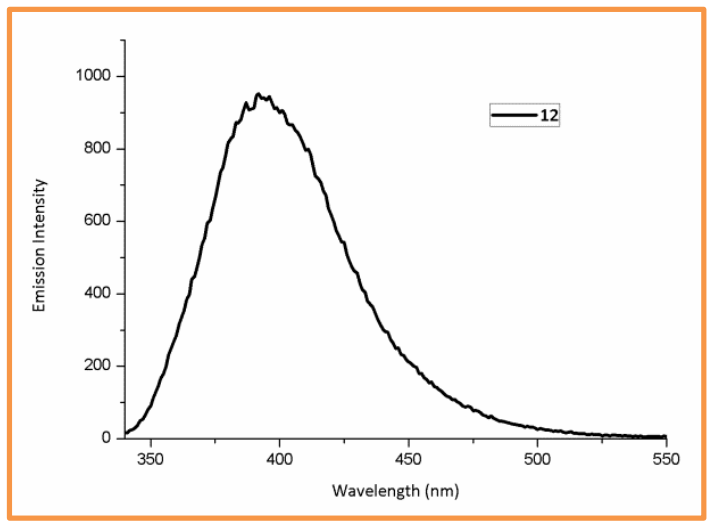

Figure S95. Fluorescence spectra of helicenoid mono alcohol, 12 
Fluorescence sensing

Fluorescence spectra for interaction of 11 with cyanide anion in different solvents:

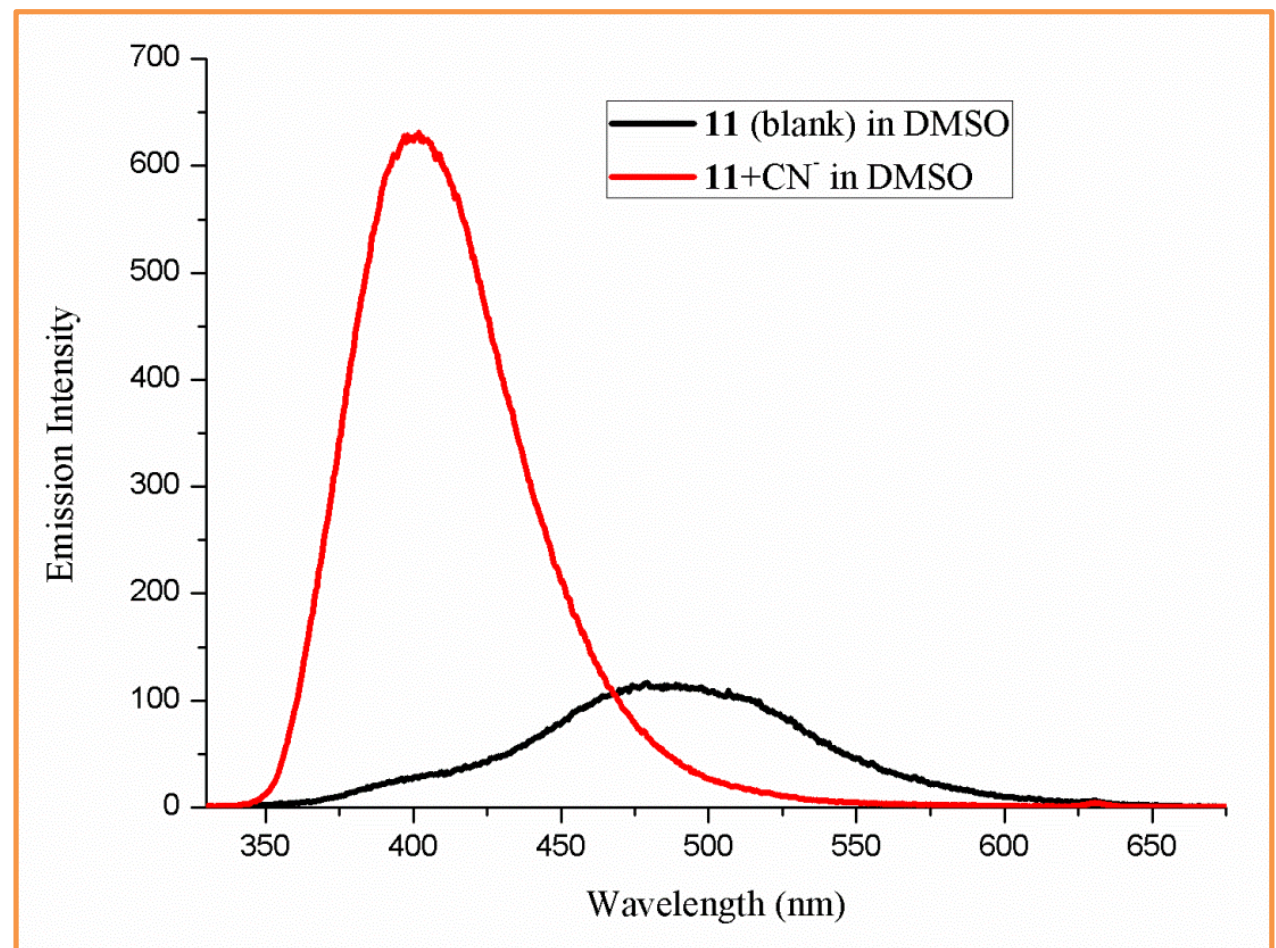

Figure S96. Fluorescence spectra for interaction of 11 with cyanide anion in DMSO (Host, $\mathbf{1 1}=4.0 \times 10^{-5} \mathrm{M}$, slit width $\left.(3,3), \lambda_{\text {exci }}=324 \mathrm{~nm}\right)$.

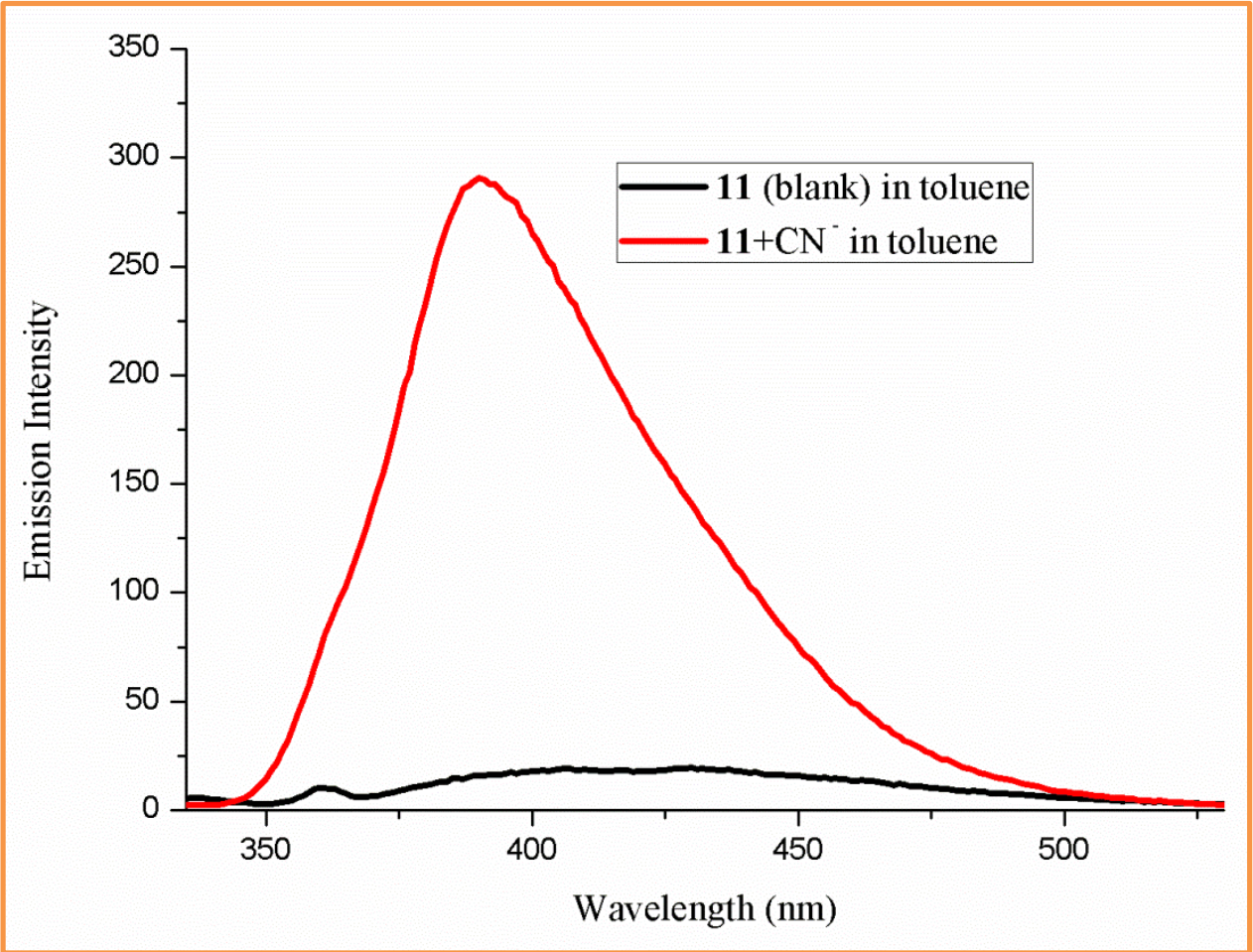

Figure S97. Fluorescence spectra for interaction of 11 with cyanide anion in toluene (Host, $11=4.0 \times 10^{-5} \mathrm{M}$, slit width $\left.(3,3), \lambda_{\text {exci }}=324 \mathrm{~nm}\right)$. 


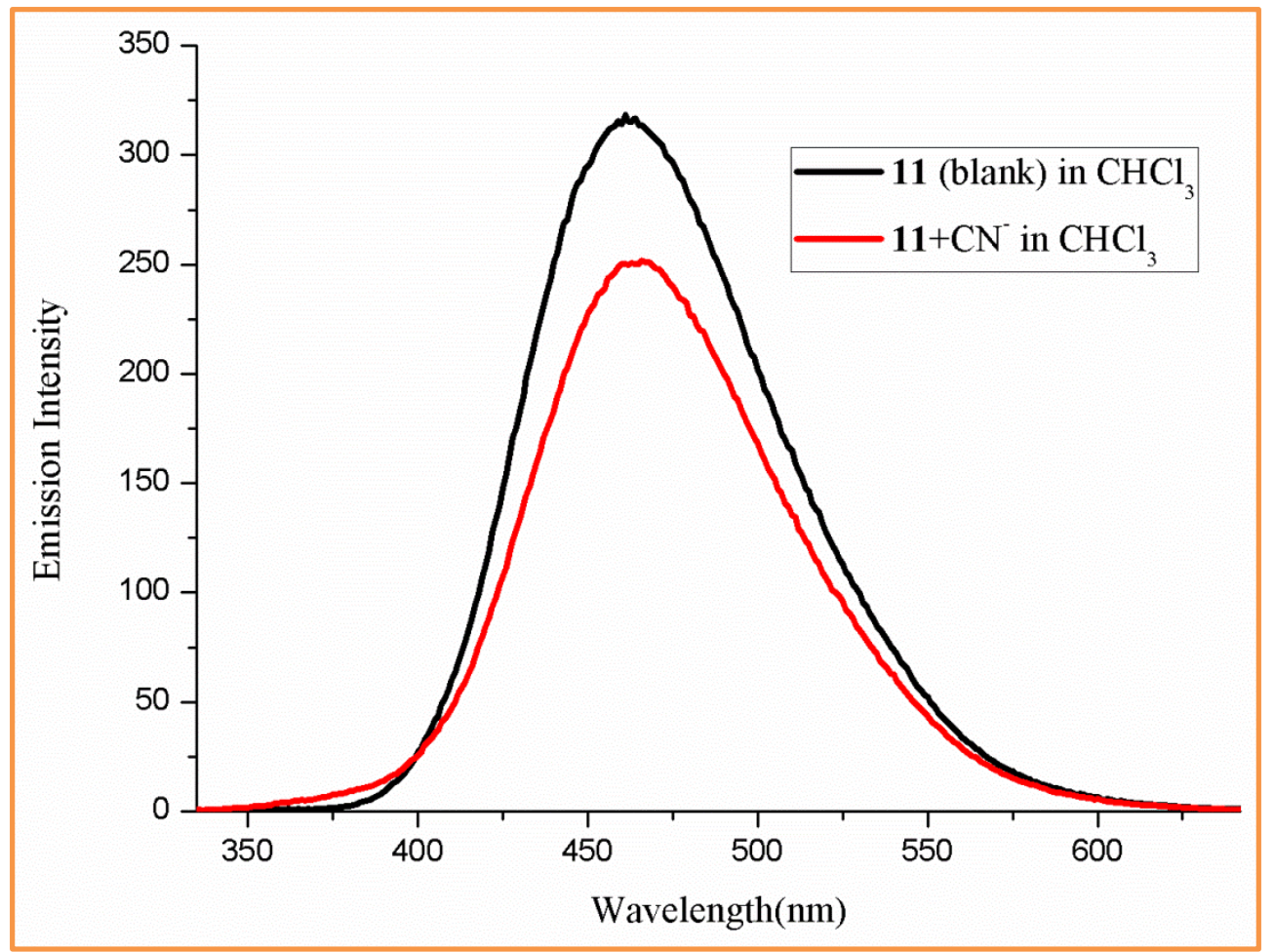

Figure S98. Fluorescence spectra for interaction of 11 with cyanide anion in chloroform (Host $\mathbf{1 1}=4.0 \times 10^{-5} \mathrm{M}$, slit width $\left.(3,3), \lambda_{\text {exci }}=324 \mathrm{~nm}\right)$.

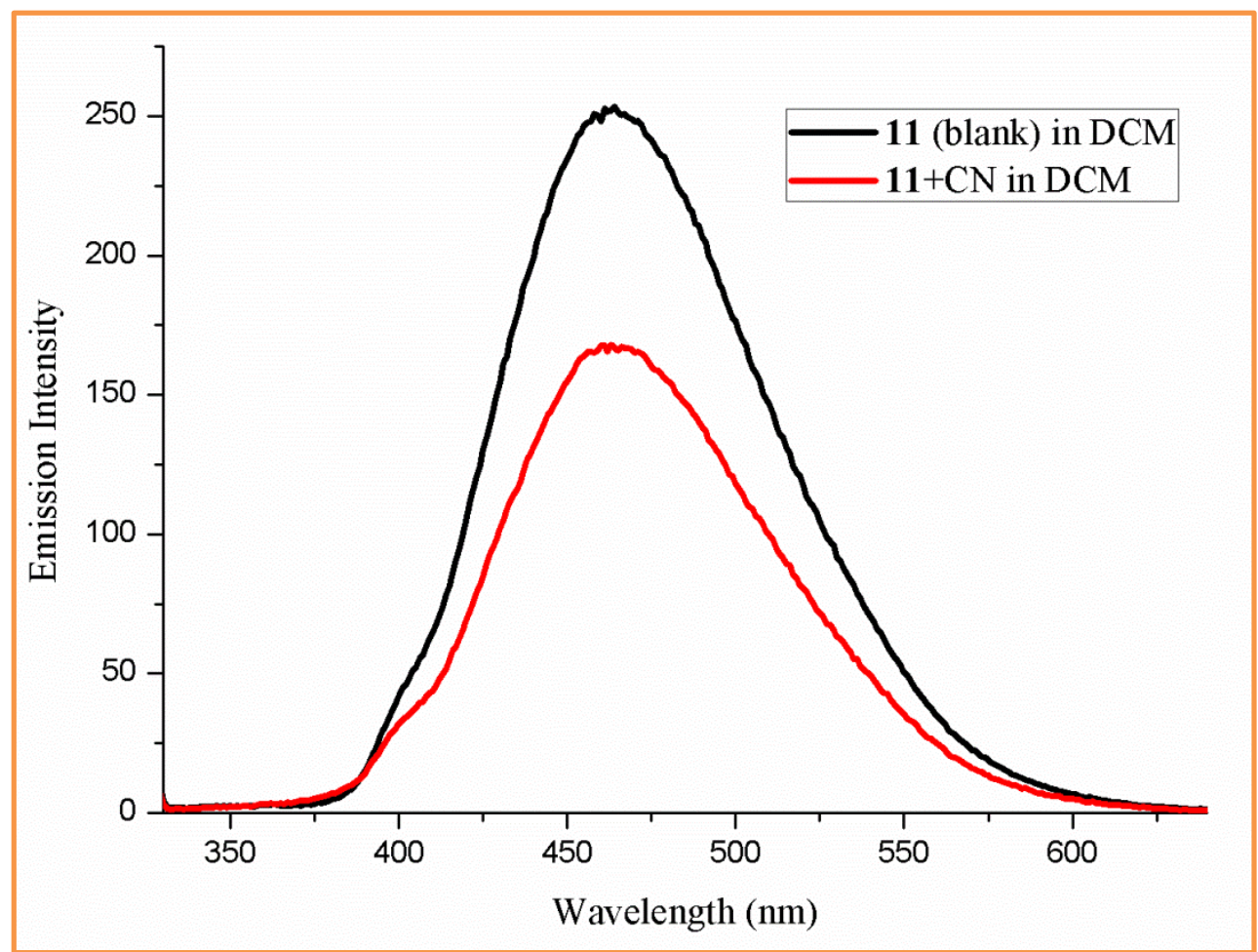

Figure S99. Fluorescence spectra for interaction of $\mathbf{1 1}$ with cyanide anion in dichloromethane (Host $11=$ $4.0 \times 10^{-5} \mathrm{M}$, slit width $\left.(3,3), \lambda_{\text {exci }}=324 \mathrm{~nm}\right)$. 


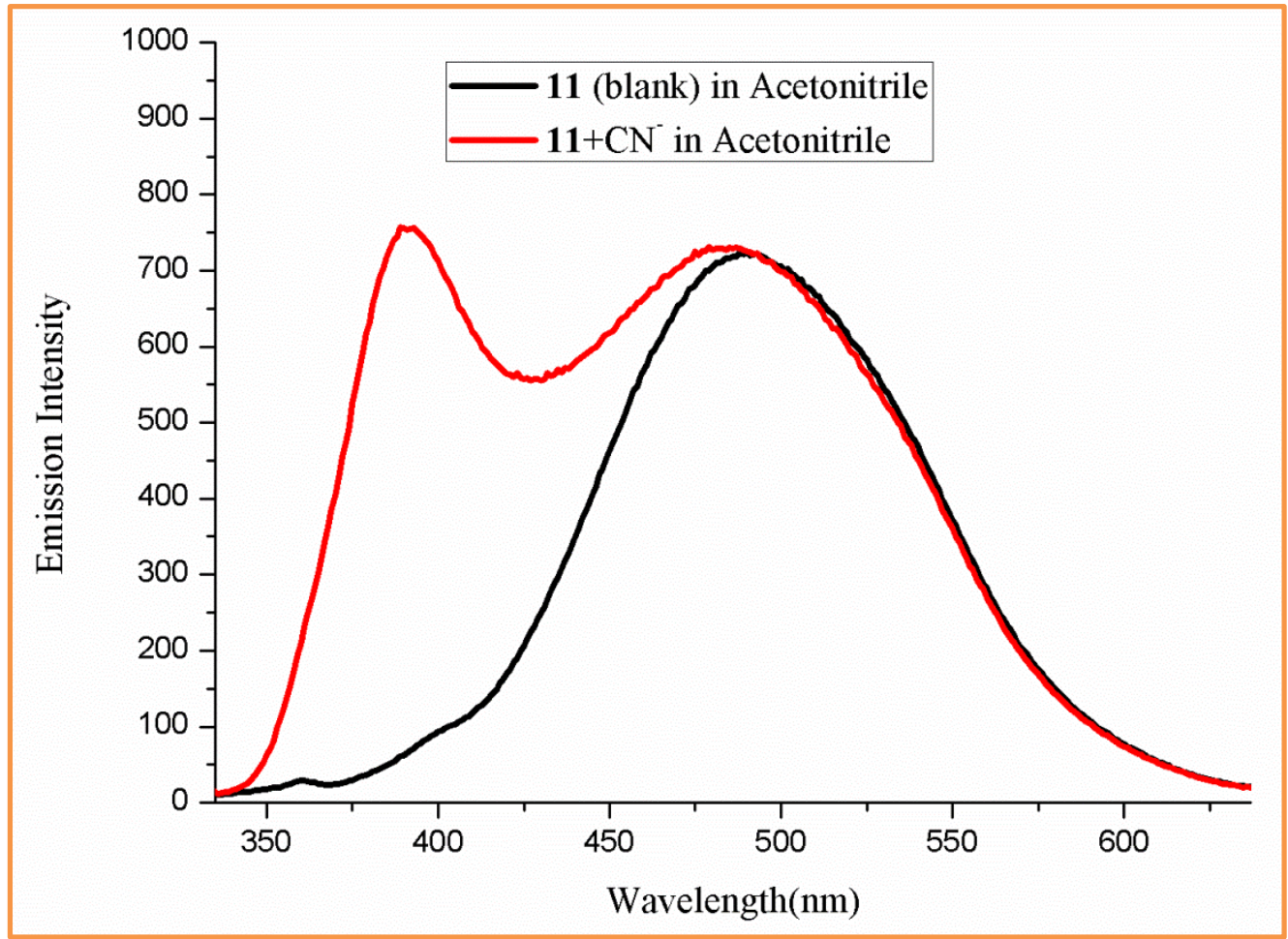

Figure S100. Fluorescence spectra for interaction of $\mathbf{1 1}$ with cyanide anion in acetonitrile (Host, $\mathbf{1 1}=4.0 \times 10^{-5}$ $M$, slit widt $\left.=h(3,3), \lambda_{\text {exci }}=324 \mathrm{~nm}\right)$.

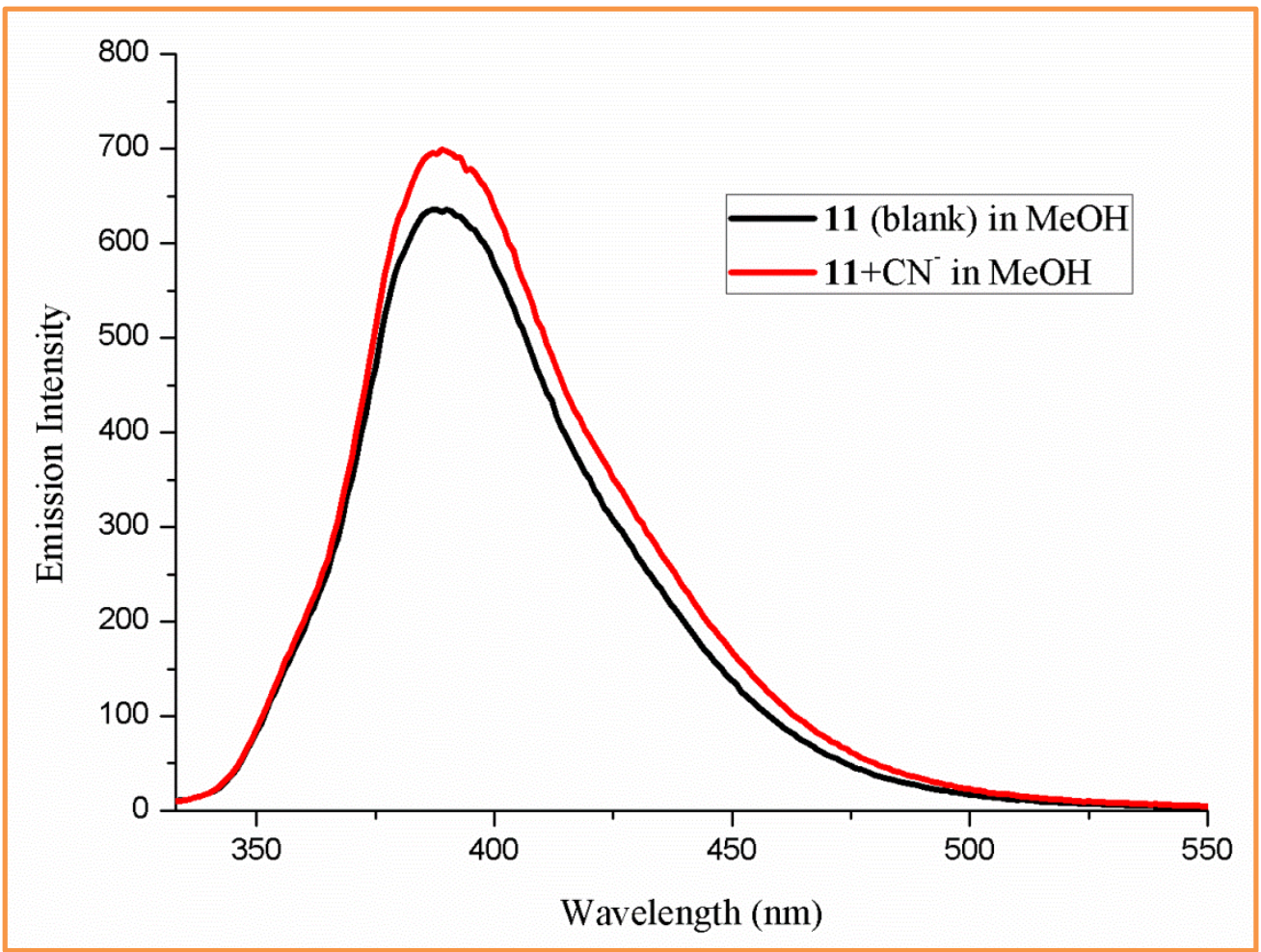

Figure S101. Fluorescence spectra for interaction of 11 with cyanide anion in methanol (Host, $\mathbf{1 1}=4.0 \times 10^{-5} \mathrm{M}$, slit width $\left.(3,3), \lambda_{\text {exci }}=324 \mathrm{~nm}\right)$. 


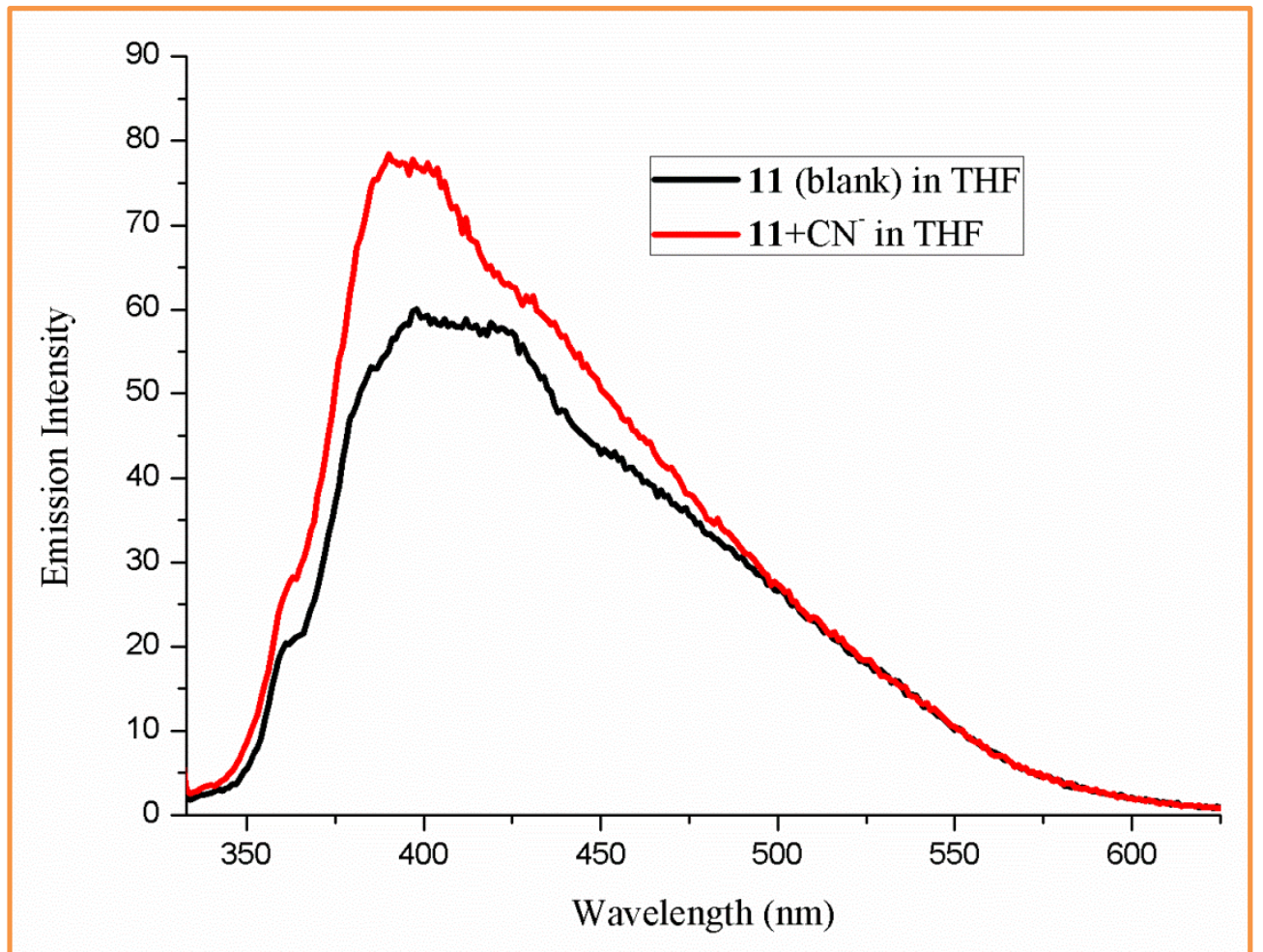

Figure S102. Fluorescence spectra for interaction of $\mathbf{1 1}$ with cyanide anion in tetrahydrofuran $($ Host, $\mathbf{1 1}=$ $4.0 \times 10^{-5} \mathrm{M}$, slit width $\left.(3,3), \lambda_{\text {exci }}=324 \mathrm{~nm}\right)$.

Fluorescence spectra for interaction of 7 with Cyanide anion in different solvents:

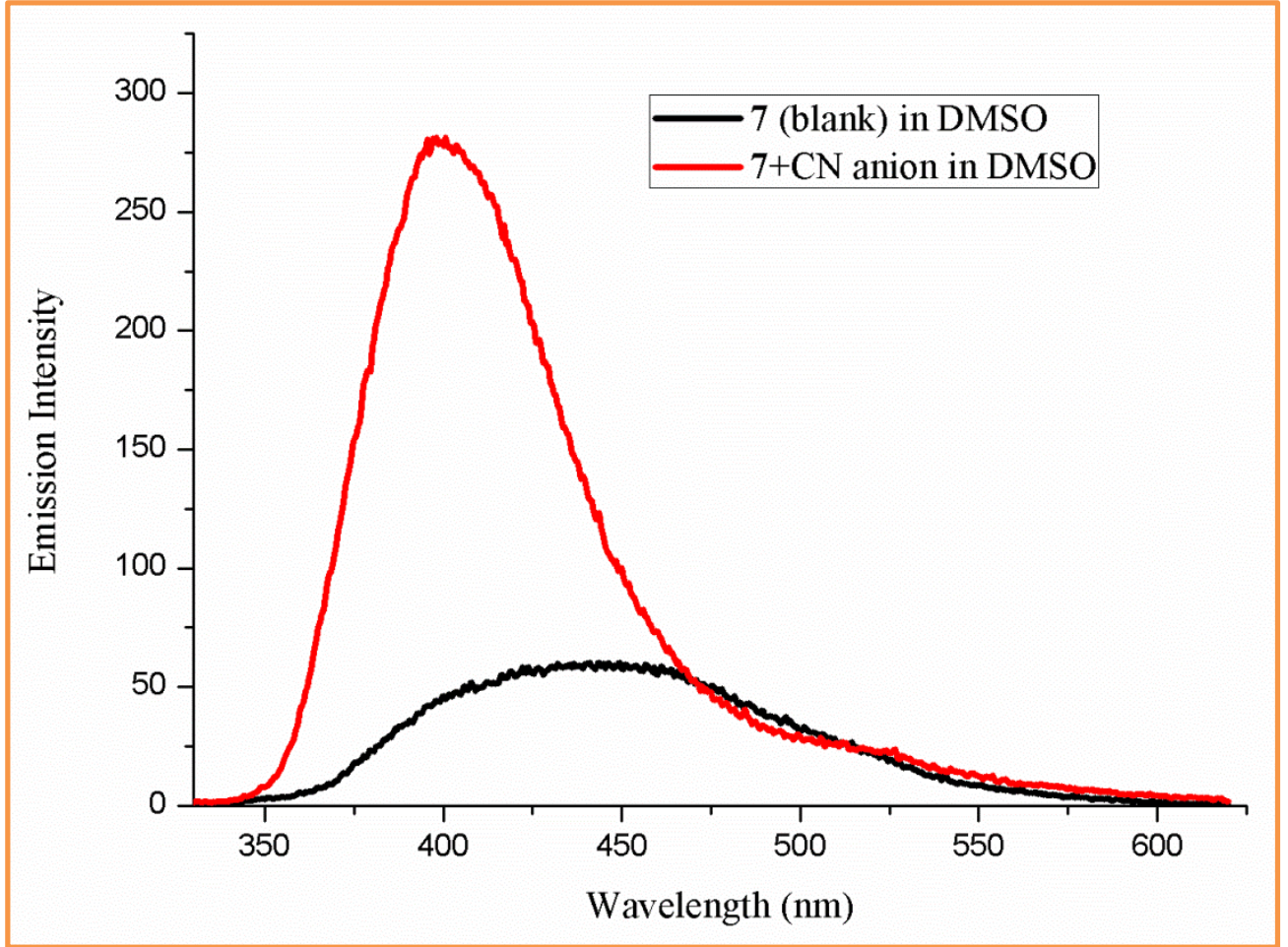

Figure S103. Fluorescence spectra for interaction of 7 with cyanide anion in DMSO (Host, $7=1.0 \times 10^{-5} \mathrm{M}$, slit width $\left.(3,3), \lambda_{\text {exci }}=328 \mathrm{~nm}\right)$. 


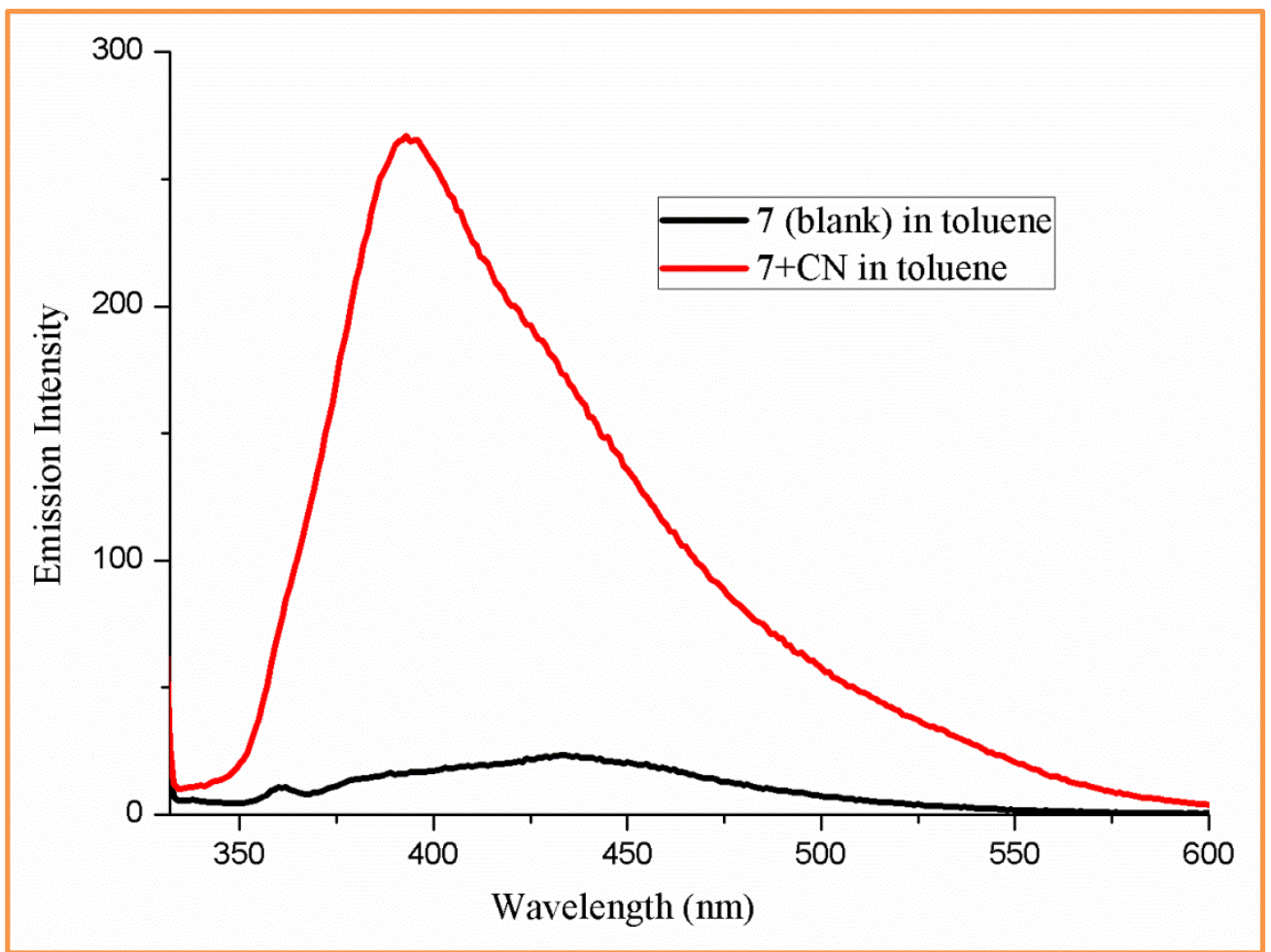

Figure S104. Fluorescence spectra for interaction of 7 with cyanide anion in toluene (Host, $7=1.0 \times 10^{-5} \mathrm{M}$, slit width $\left.(3,3), \lambda_{\text {exci }}=328 \mathrm{~nm}\right)$.

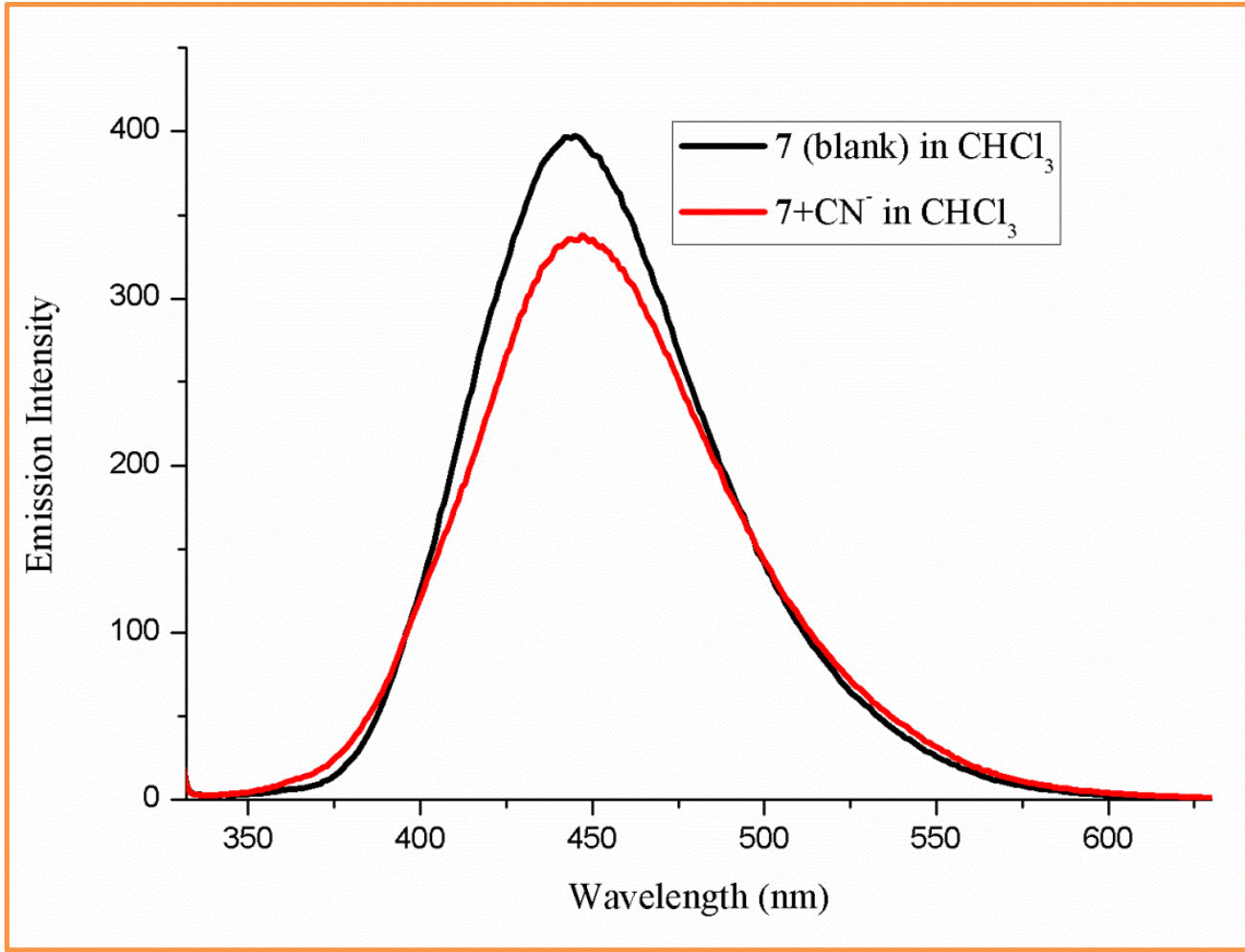

Figure S105. Fluorescence spectra for interaction of 7 with cyanide anion in chloroform (Host, $7=1.0 \times 10^{-5} \mathrm{M}$, slit width $\left.(3,3), \lambda_{\text {exci }}=328 \mathrm{~nm}\right)$. 


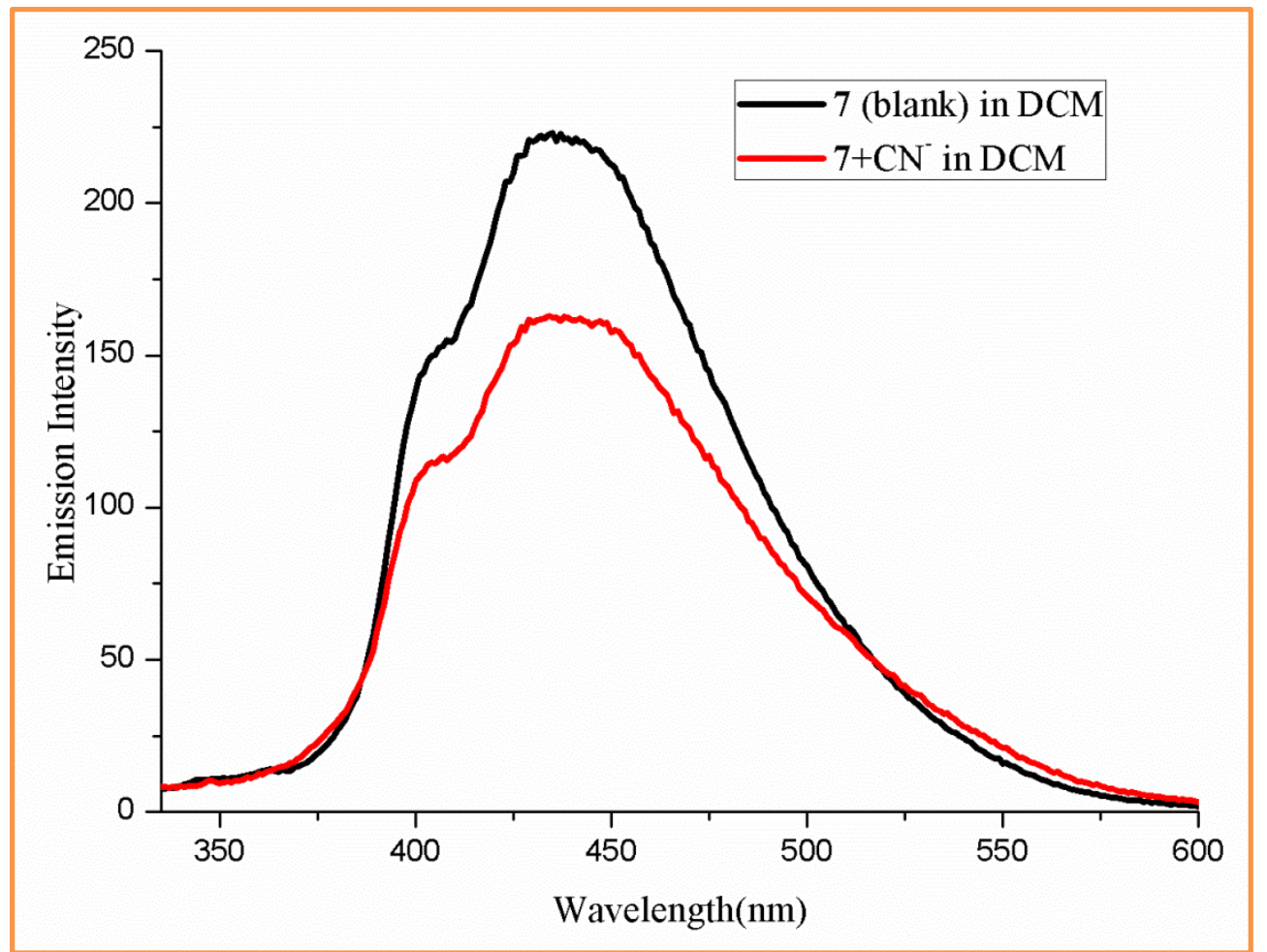

Figure S106. Fluorescence spectra for interaction of 7 with cyanide anion in dichloromethane (Host, $7=$ $1.0 \times 10^{-5} \mathrm{M}$, slit width $\left.(3,3), \lambda_{\text {exci }}=328 \mathrm{~nm}\right)$.

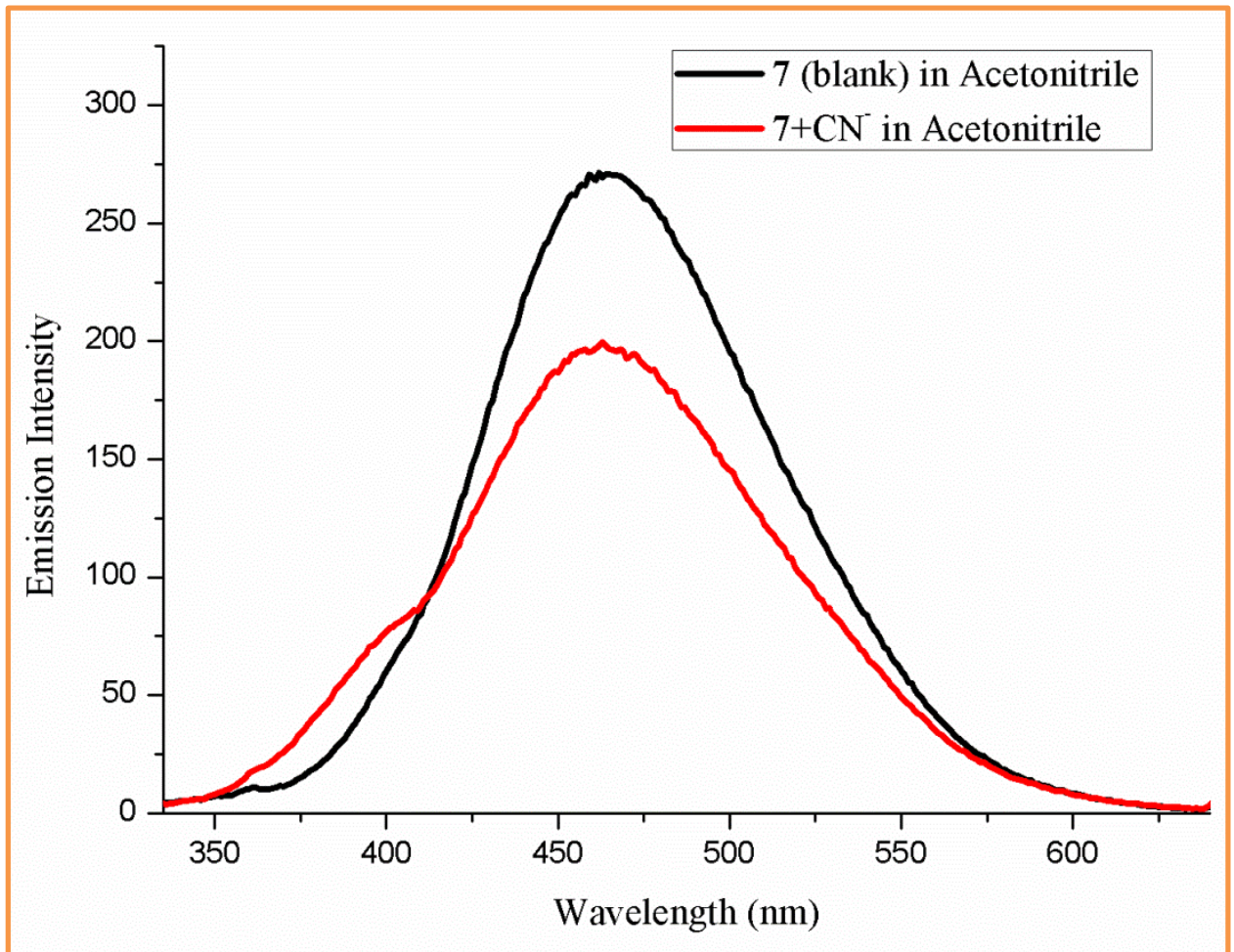

Figure S107. Fluorescence spectra for interaction of 7 with cyanide anion in acetonitrile (Host, $7=1.0 \times 10^{-5} \mathrm{M}$, slit width $\left.(3,3), \lambda_{\text {exci }}=328 \mathrm{~nm}\right)$. 


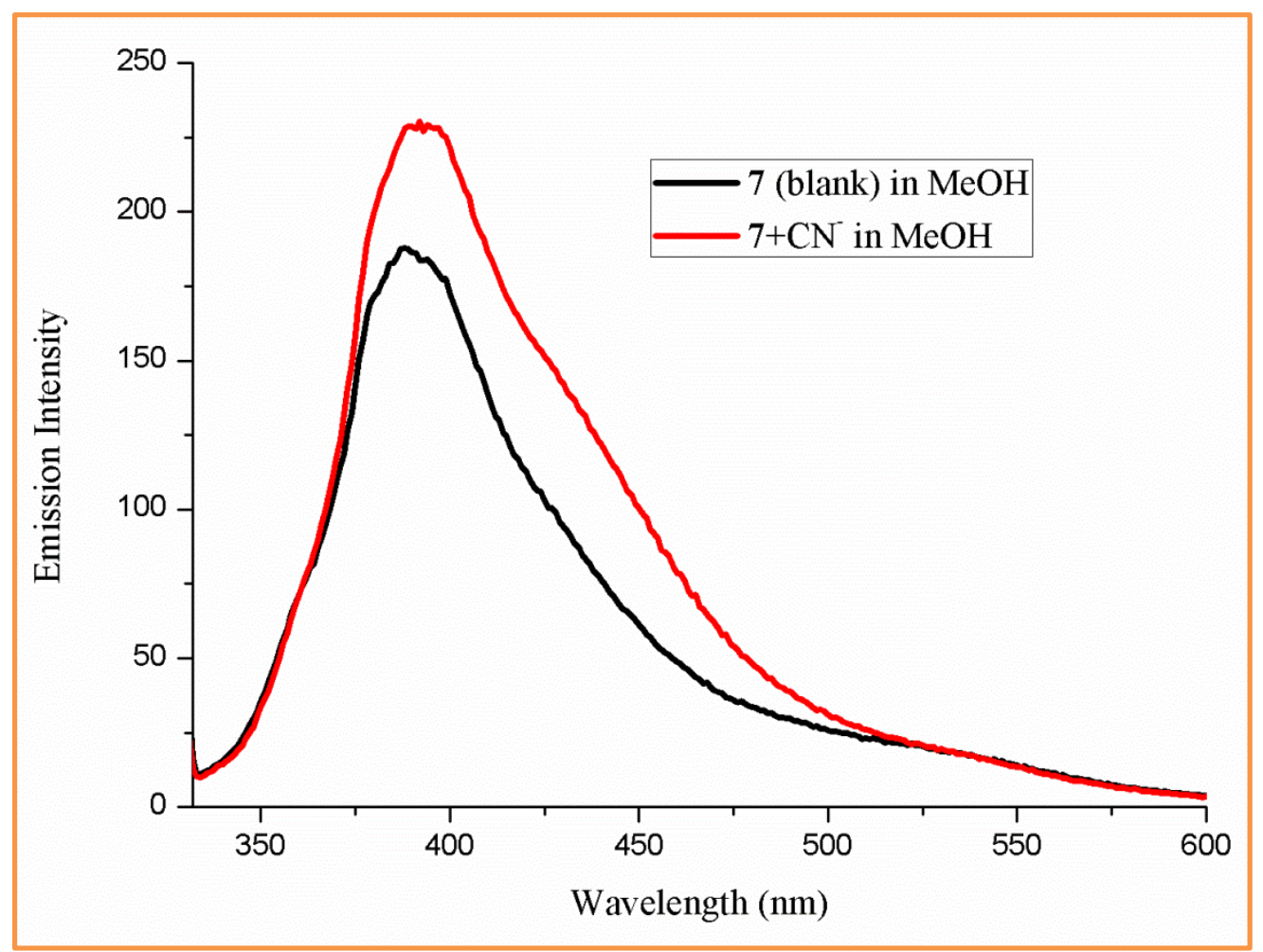

Figure S108. Fluorescence spectra for interaction of 7 with cyanide anion in methanol (Host, $7=1.0 \times 10^{-5} \mathrm{M}$, slit width $\left.(3,3), \lambda_{\text {exci }}=328 \mathrm{~nm}\right)$.

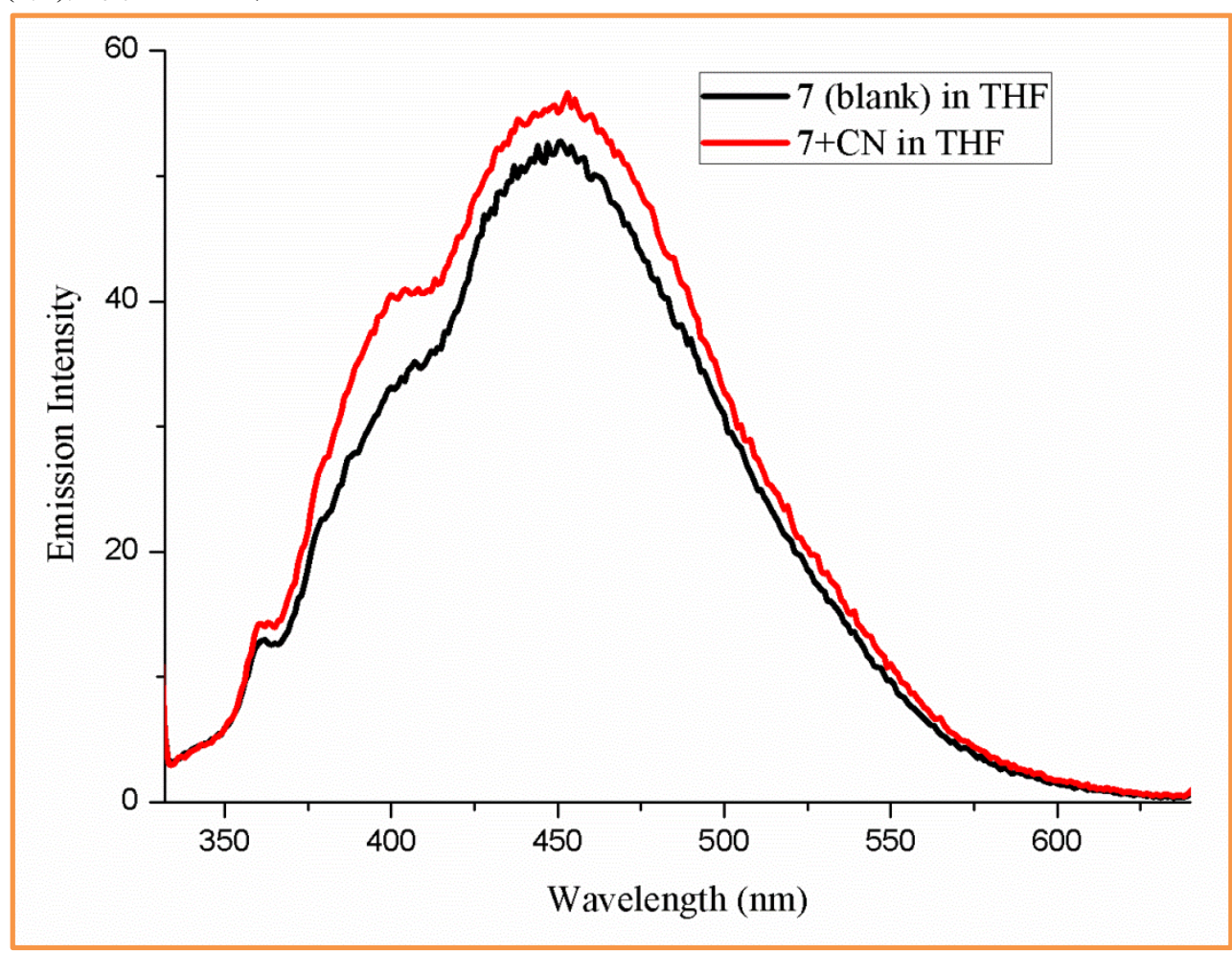

Figure S109. Fluorescence spectra for interaction of $7\left(4.0 \times 10^{-5} \mathrm{M}\right)$ with cyanide anion in tetrahydrofuran (Host, $7=1.0 \times 10^{-5} \mathrm{M}$, slit width $\left.(3,3), \lambda_{\text {exci }}=328 \mathrm{~nm}\right)$. 


\section{Calculation of Limit of Detection (LOD):}

Limit of detection (LOD) for the cyanide anion was calculated by linear fiting plot and using following equation:

$$
\mathrm{LOD}=\mathrm{K} \times \delta / \mathrm{S}
$$

Where, $\delta=$ Standard deviation of the host, determined from the following equation

$$
\delta=\sqrt{\frac{\sum\left(F_{0}-\bar{F}_{0}\right)^{2}}{N-1}}
$$

$\mathrm{N}=$ Number of readings taken for host, $\mathrm{F}_{0}=$ The highest emission intesity, $\bar{F}_{0}=$ Mean of readings

The standard deviation simply can be calculated from the standard formula provided in the excel sheet. We have prepared three different solutions of the host of particular concentration and recorded its fluorescence spectra. The highest emission intesity $\left(\mathrm{F}_{0}\right)$ obtained from flourescene data is used for the calculation of standard deviation.

$\mathrm{S}=$ Slope obtained of linear equation

$\mathrm{K}=$ Constant $=3$

(i) LOD for titration of 11 and cyanide anion in DMSO:

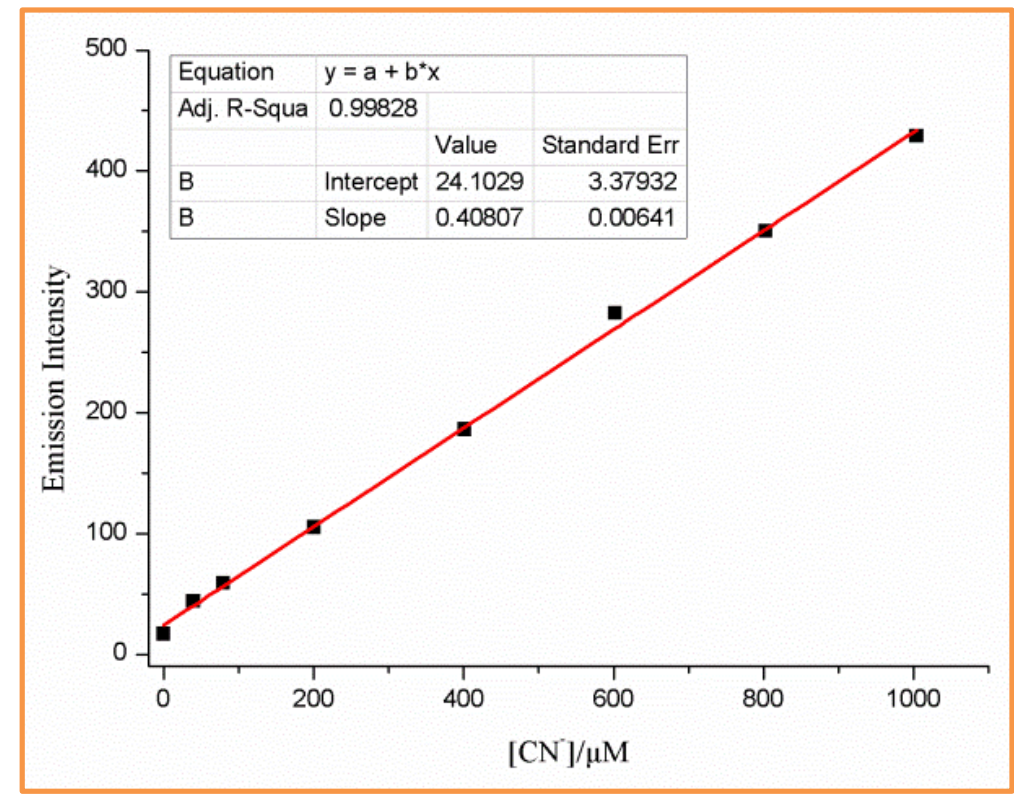

Figure S110. Plot of emission intensity of $\mathbf{1 1} \mathrm{Vs}\left[\mathrm{CN}^{-}\right]$in DMSO.

The linear equation, $\mathrm{Y}=0.302 \times \mathrm{X}+61.82$

$\mathrm{S}=0.302, \mathrm{~K}=3, \delta=0.003055$

Thus, $\mathrm{LOD}=3 \times 0.003055 / 0.408$

$$
\mathrm{LOD}=0.0225 \mu \mathrm{M}
$$


(ii) LOD for titration of 11 and cyanide anion in toluene

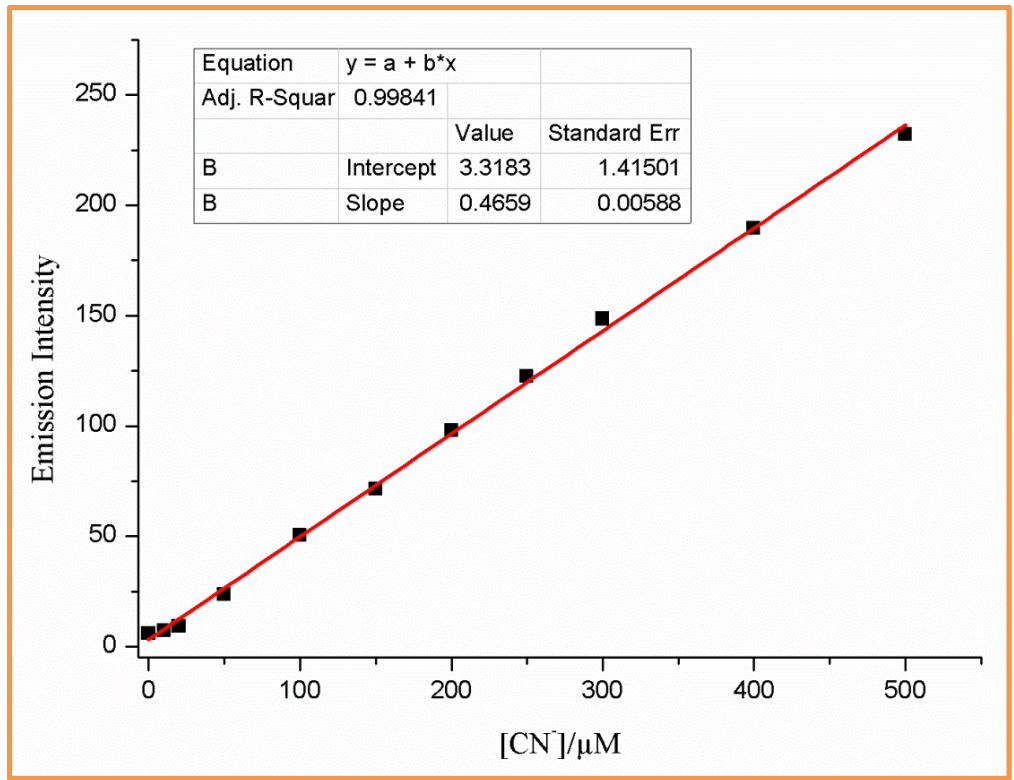

Figure S111. Plot of emission intensity of $\mathbf{1 1 ~} \mathrm{Vs}\left[\mathrm{CN}^{-}\right]$in toluene.

The linear equation, $\mathrm{Y}=0.4659 \times \mathrm{X}+3.31$

$\mathrm{S}=0.4659, \mathrm{~K}=3, \delta=0.002517$

Thus, $\mathrm{LOD}=3 \times 0.002517 / 0.04659$

$$
\mathrm{LOD}=0.0162 \mu \mathrm{M}
$$

\section{(iii) LOD for titration of 7 and cyanide anion in DMSO}

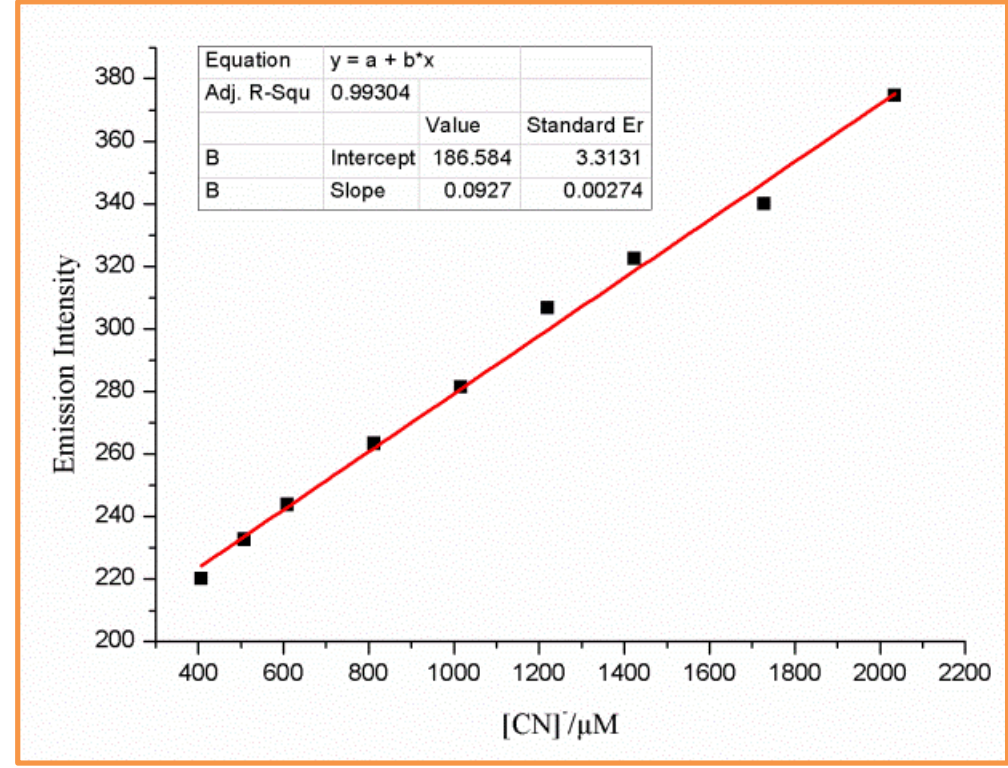

Figure S112. Plot of emission intensity of $7 \mathrm{Vs}\left[\mathrm{CN}^{-}\right]$in DMSO.

The linear equation, $\mathrm{Y}=0.0632 \times \mathrm{X}+219$ 
$\mathrm{S}=0.0632, \mathrm{~K}=3, \delta=0.00854$

Thus, $\mathrm{LOD}=3 \times 0.00854 / 0.0927=0.2764 \mu \mathrm{M}$

(iv)

\section{LOD for titration of HDA, 85 and cyanide anion in toluene}

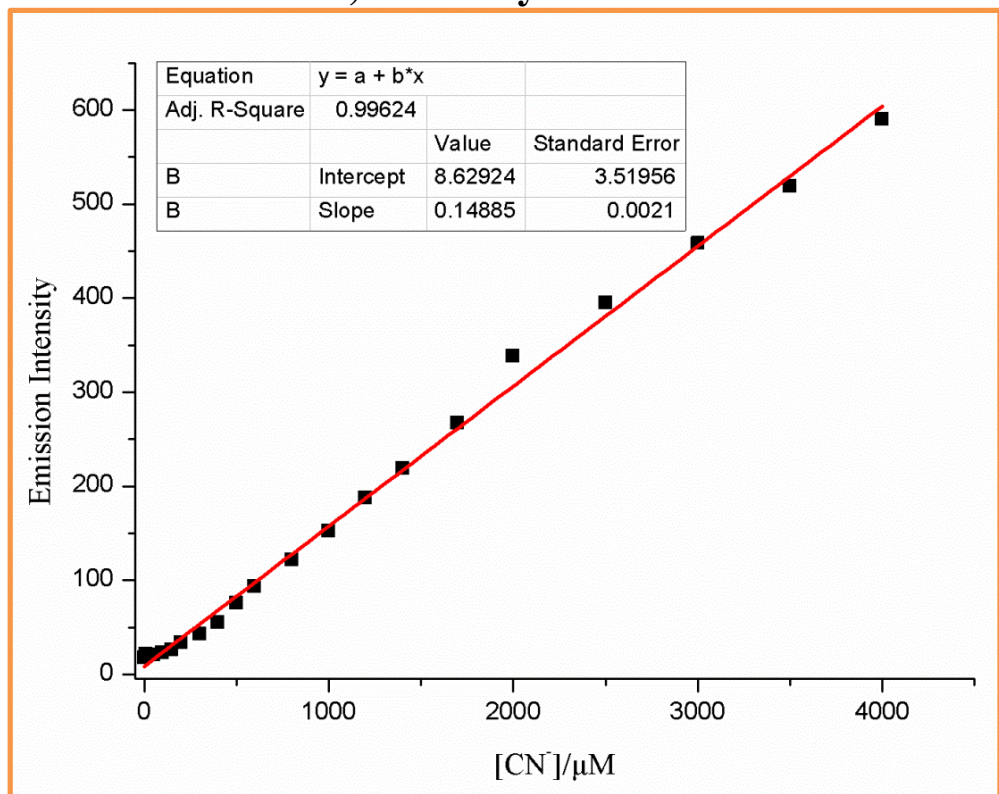

Figure S113. Plot of emission intensity of $7 \mathrm{Vs}\left[\mathrm{CN}^{-}\right]$in toluene.

The linear equation, $\mathrm{Y}=0.1488 \times \mathrm{X}+219$

$\mathrm{S}=0.1488, \mathrm{~K}=3, \delta=0.009713$

Thus, $\mathrm{LOD}=3 \times 0.009713 / 0.1488=0.195 \mu \mathrm{M}$

Job's plot analysis using UV-vis spectroscopy (Continuous variation method)

(i) For 11 and $\mathrm{CN}$ titration in DMSO

A series of solutions containing host, helicenoid mono aldehyde, $\mathbf{1 1}$ with cyanide anion were prepared such that the sum of the total host and guest concentration remained constant $\left(1 \times 10^{-}\right.$ ${ }^{5} \mathrm{M}$ ) in DMSO solvent. The mole fraction (X) of guest cyanide was varied from 0.0, 0.1, 0.3, $0.5,0.7,0.9,1.0$. The corrected absorption (absorbance at $254 \mathrm{~nm} \times$ mole fraction (X) of guest cyanide) was plotted against molar fraction (X) of the guest $\mathrm{CN}$ anion, which confirmed 1:1 stoichiometry for $11: \mathrm{CN}^{-}$

\begin{tabular}{|r|r|r|r|}
\hline $\begin{array}{c}\text { Mole fraction } \\
\text { of Guest } \\
(\mathbf{X})\end{array}$ & $\begin{array}{l}\text { Mole fraction } \\
\text { of Host, 89 } \\
\text { (Y) }\end{array}$ & \multicolumn{1}{|c|}{$\begin{array}{c}\text { Absorbance } \\
\text { at 313 } \mathbf{~ m}\end{array}$} & \multicolumn{1}{c|}{$\begin{array}{c}\text { Corrected } \\
\text { absorbance } \\
\text { (Abs. * X })\end{array}$} \\
\hline 1.0 & 0.0 & 0.0 & 0.0 \\
\hline 0.9 & 0.1 & 0.037 & 0.0333 \\
\hline 0.7 & 0.3 & 0.095 & 0.0665 \\
\hline 0.5 & 0.5 & 0.163 & 0.0815 \\
\hline 0.3 & 0.7 & 0.221 & 0.0663 \\
\hline 0.1 & 0.9 & 0.274 & 0.0274 \\
\hline 0.0 & 1.0 & 0.291 & 0.0 \\
\hline
\end{tabular}




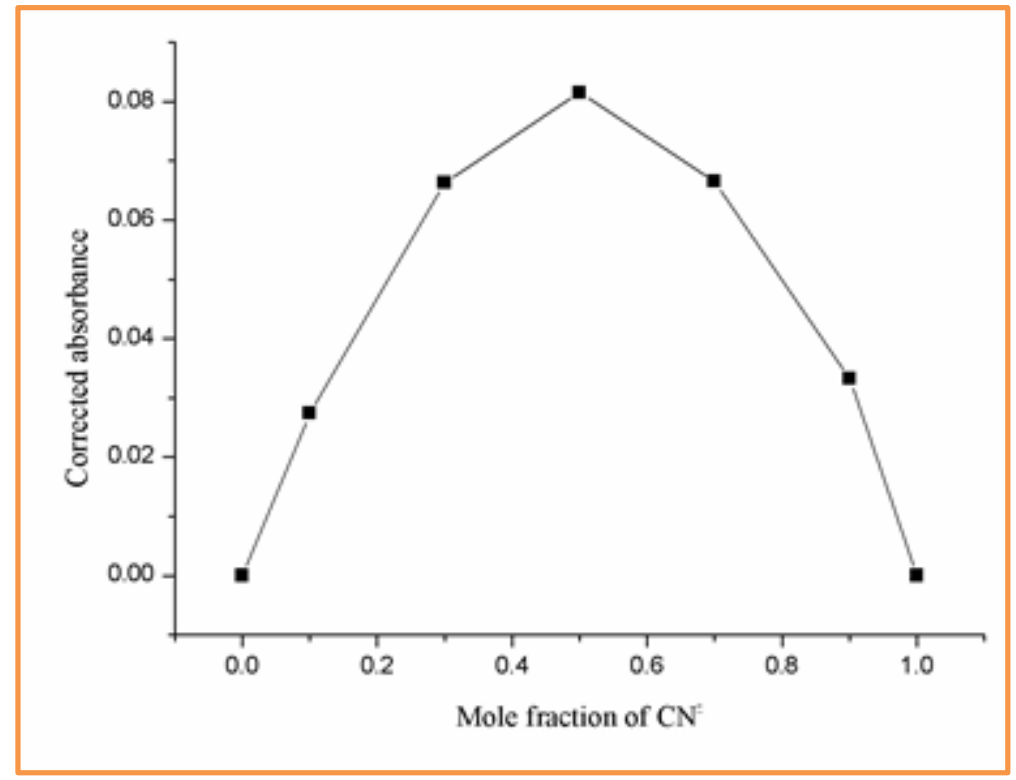

Figure S114. Jobs plot for interaction of $\mathbf{1 1}$ and cyanide anion in DMSO.

\section{(ii) For 7 and $\mathrm{CN}$ titration in DMSO}

A series of solutions containing host, helicenoid di-aldehyde, 7 with cyanide anion were prepared such that sum of the total host and guest concentration remained constant $\left(1 \times 10^{-5} \mathrm{M}\right)$ in DMSO solvent. The mole fraction (X) of guest cyanide was varied from 0.0, 0.1, 0.3, 0.5, $0.67,0.9,1.0$. The corrected absorption (absorbance at $304 \mathrm{~nm} \times$ mole fraction (X) of guest cyanide) was plotted against the molar fraction (X) of the guest cyanide, which confirmed 1:2 stoichiometry for 7: $\mathrm{CN}^{-}$

\begin{tabular}{|c|c|c|c|}
\hline $\begin{array}{c}\text { Mole fraction } \\
\text { of Guest } \\
(\mathbf{X})\end{array}$ & $\begin{array}{c}\text { Mole fraction } \\
\text { of Host, 85 } \\
(\mathbf{Y})\end{array}$ & $\begin{array}{c}\text { Absorbance } \\
\text { at 304 } \mathbf{~ n m}\end{array}$ & $\begin{array}{c}\text { Corrected } \\
\text { absorbance } \\
(\text { Abs. } * \mathbf{X})\end{array}$ \\
\hline 1.0 & 0.0 & 0.0 & 0.0 \\
\hline 0.9 & 0.1 & 0.89 & 0.0712 \\
\hline 0.67 & 0.33 & 0.117 & 0.07839 \\
\hline 0.5 & 0.5 & 0.139 & 0.0695 \\
\hline 0.3 & 0.7 & 0.18 & 0.0594 \\
\hline 0.1 & 0.9 & 0.201 & 0.402 \\
\hline 0.0 & 1.0 & 0.228 & 0.0 \\
\hline
\end{tabular}




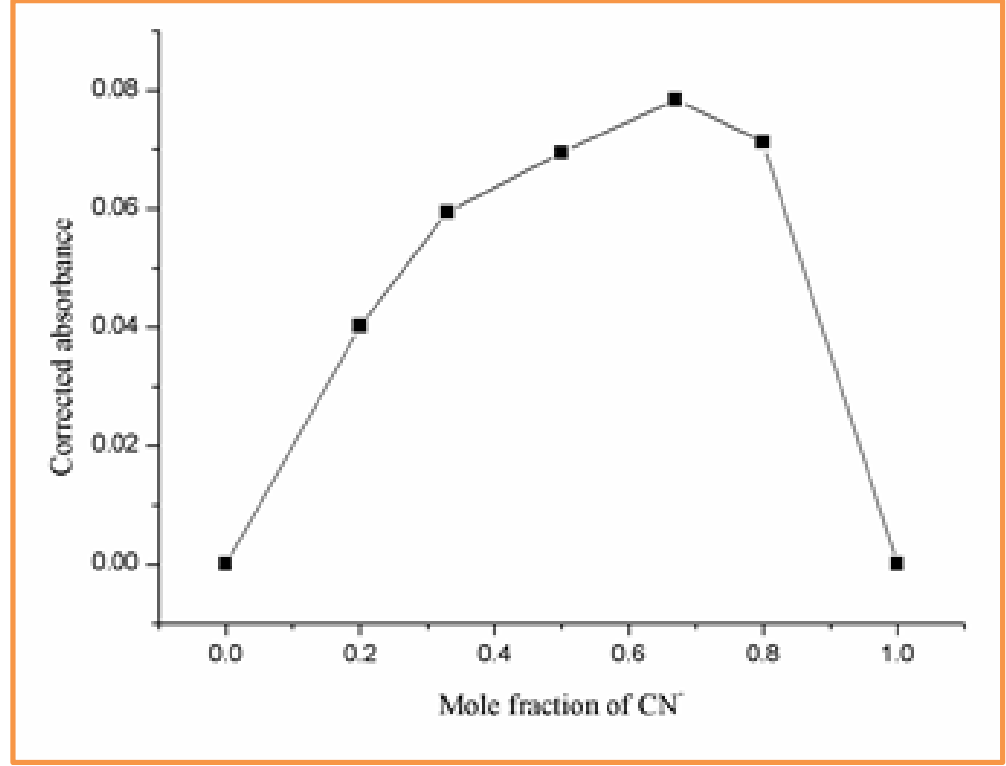

Figure S115. Jobs plot for interaction of 7 and cyanide anion in DMSO.

Fluorescence spectra for interaction of $\mathbf{1 5}$ and furfural with cyanide anion:

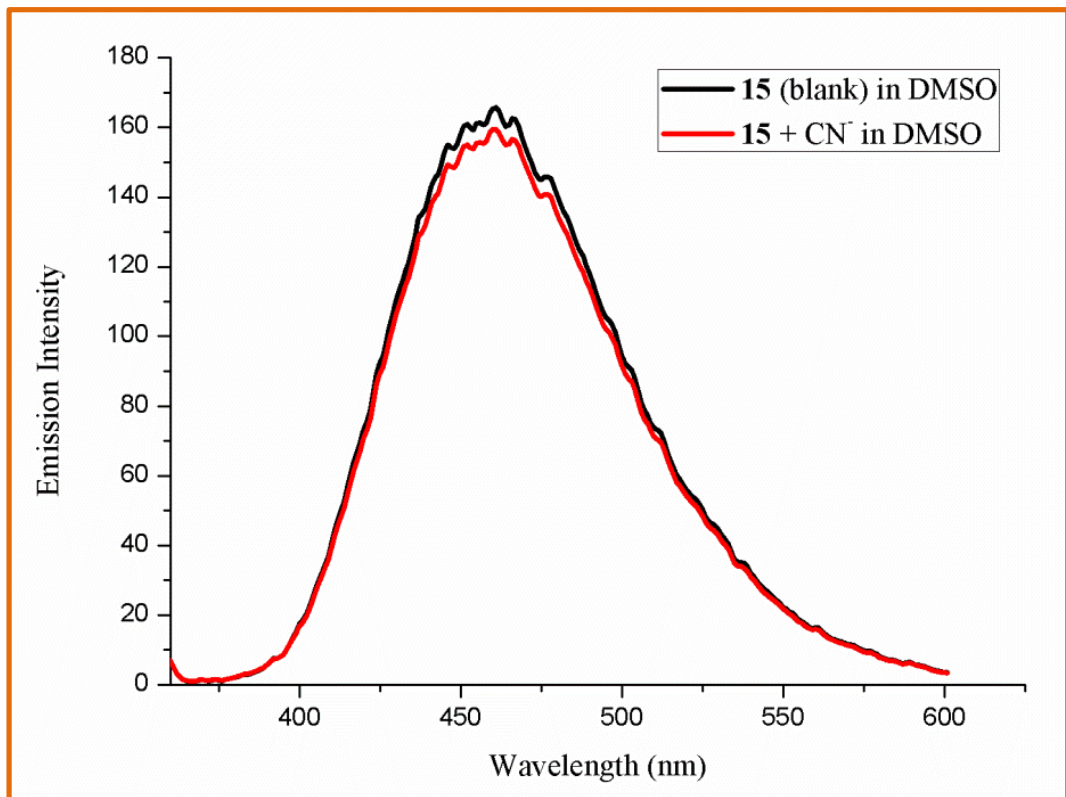

Figure S116. Fluorescence spectra for interaction of 15 with cyanide anion in DMSO (Host, $15=1.0 \times 10^{-5} \mathrm{M}$, slit width $\left.(3,3), \lambda_{\text {exci }}=358 \mathrm{~nm}\right)$. 


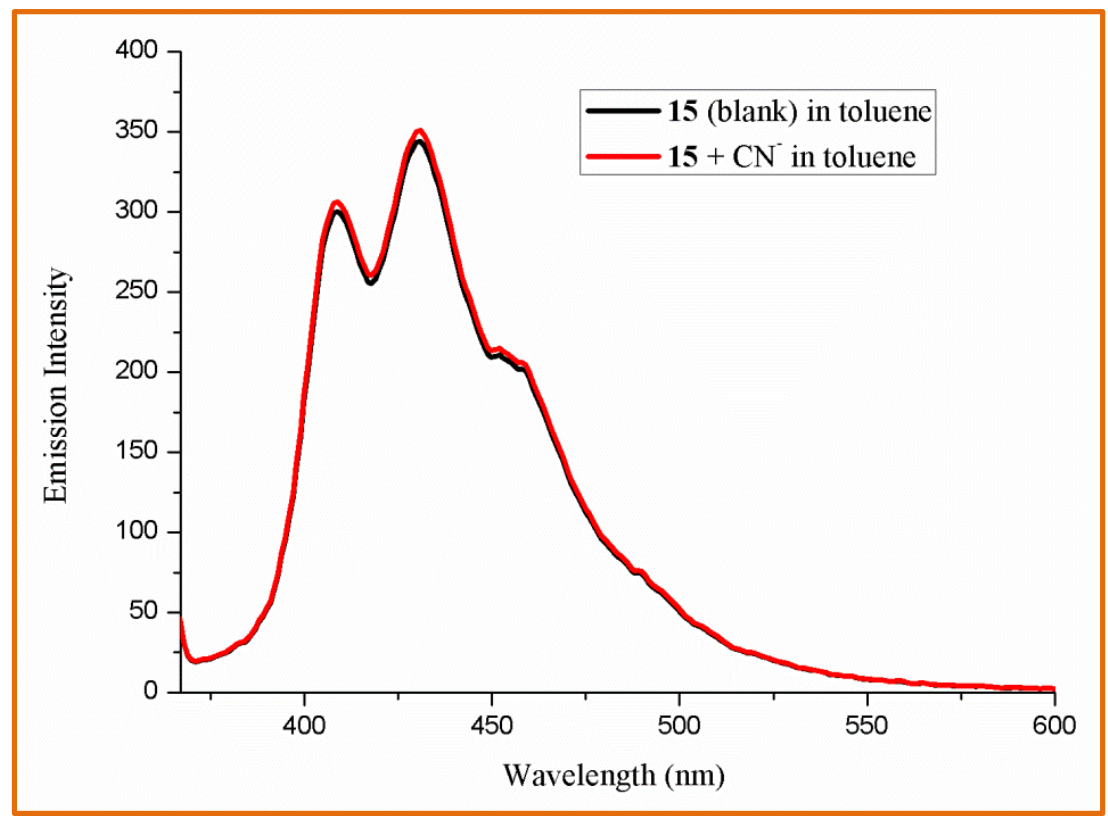

Figure S117. Fluorescence spectra for interaction of $\mathbf{1 5}$ with cyanide anion in toluene (Host, $\mathbf{1 5}=1.0 \times 10^{-5} \mathrm{M}$, slit width $\left.(3,3), \lambda_{\text {exci }}=358 \mathrm{~nm}\right)$.

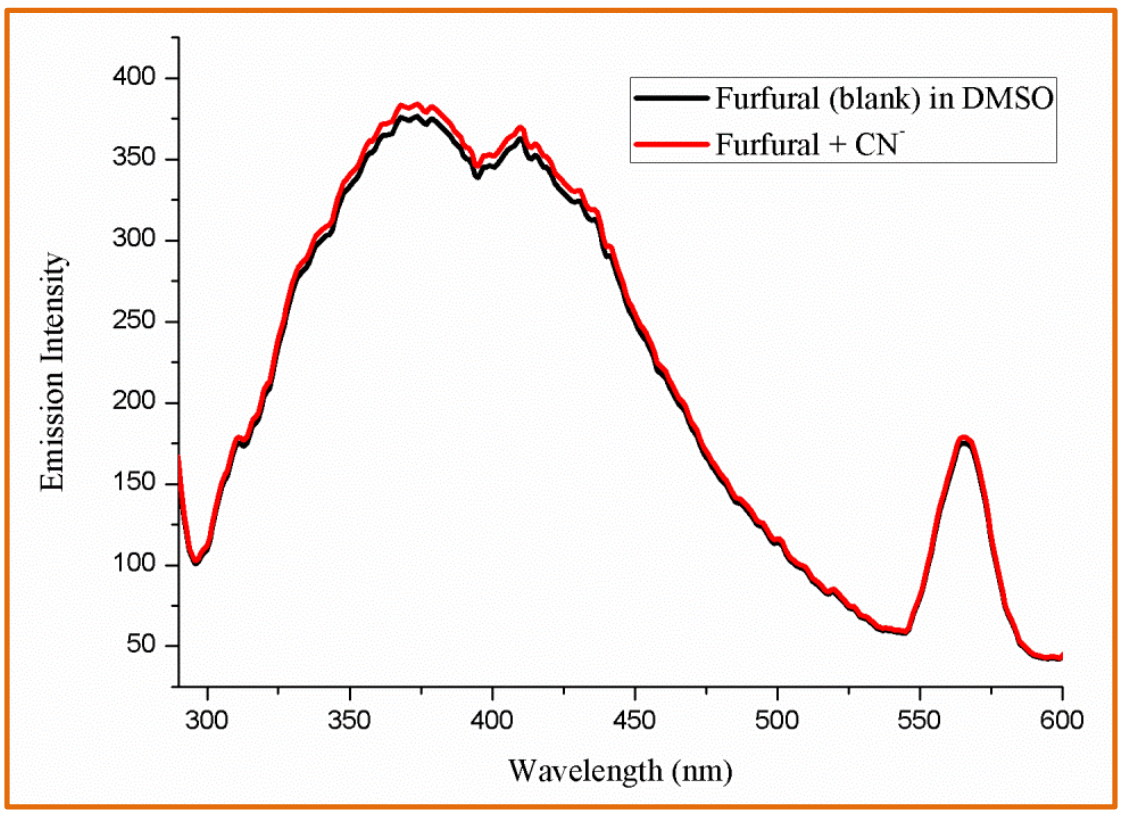

Figure S118. Fluorescence spectra for interaction of furfural with cyanide anion in DMSO (Host, furfural = $1.0 \times 10^{-5} \mathrm{M}$, slit width $\left.(3,3), \lambda_{\text {exci }}=283 \mathrm{~nm}\right)$. 


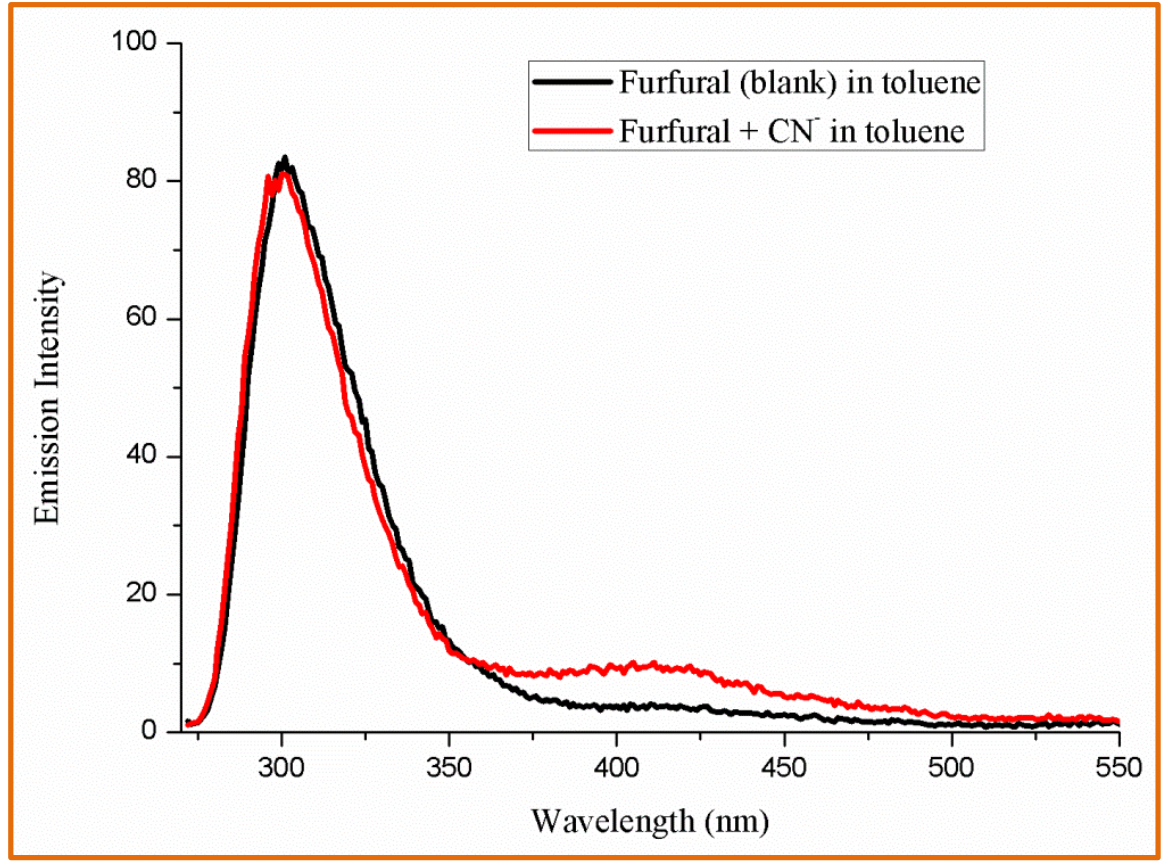

Figure S119. Fluorescence spectra for interaction of furfural with cyanide anion in toluene (Host, furfural = $1.0 \times 10^{-5} \mathrm{M}$, slit width $\left.(3,3), \lambda_{\text {exci }}=283 \mathrm{~nm}\right)$.

\section{UV study of helicenoid aldehydes towards cyanide anion sensing:}

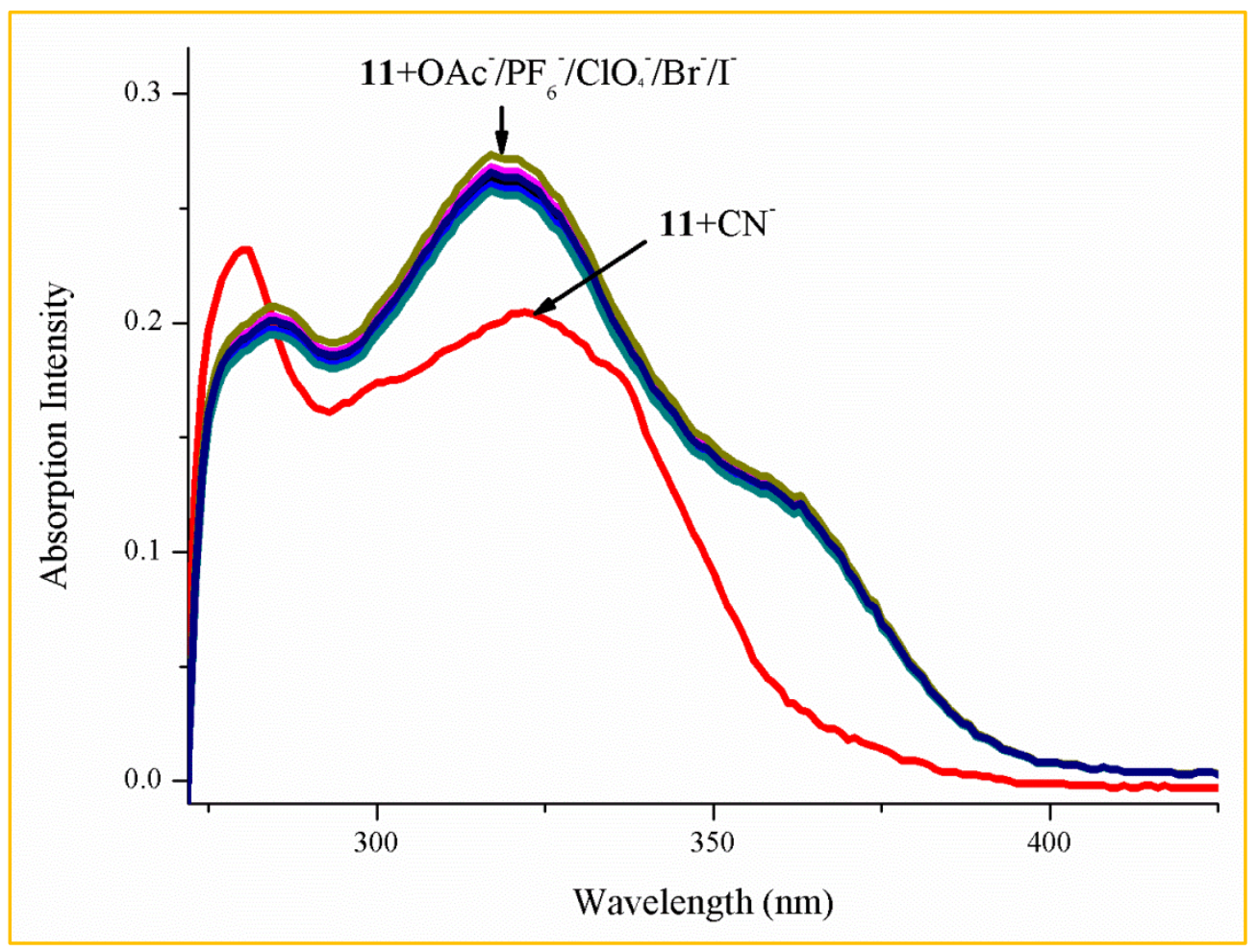

Figure S120. UV spectra of 11 containing various anion 100 eq. (as tetrabutylmmonium salts) in DMSO solution. (Host $11, \mathrm{C}=1.0 \times 10^{-5} \mathrm{M}$ ). 


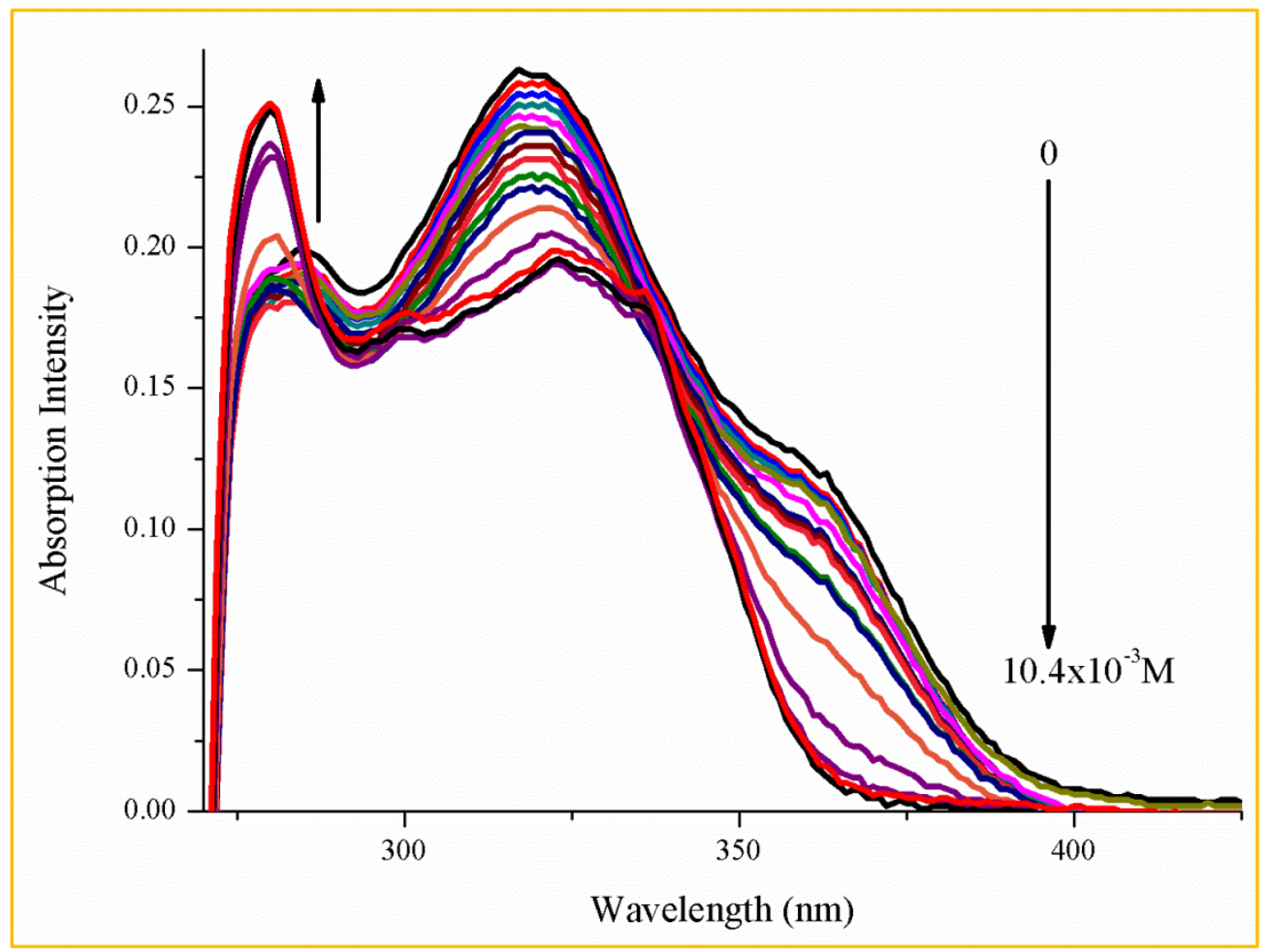

Figure S121. UV spectra of 11 upon gradual increase of $\mathrm{CN}^{-}\left(0-10.4 \times 10^{-3} \mathrm{M}\right)$ in DMSO. $\left(\right.$ Host $\left.\mathrm{C}=1.0 \times 10^{-5} \mathrm{M}\right)$

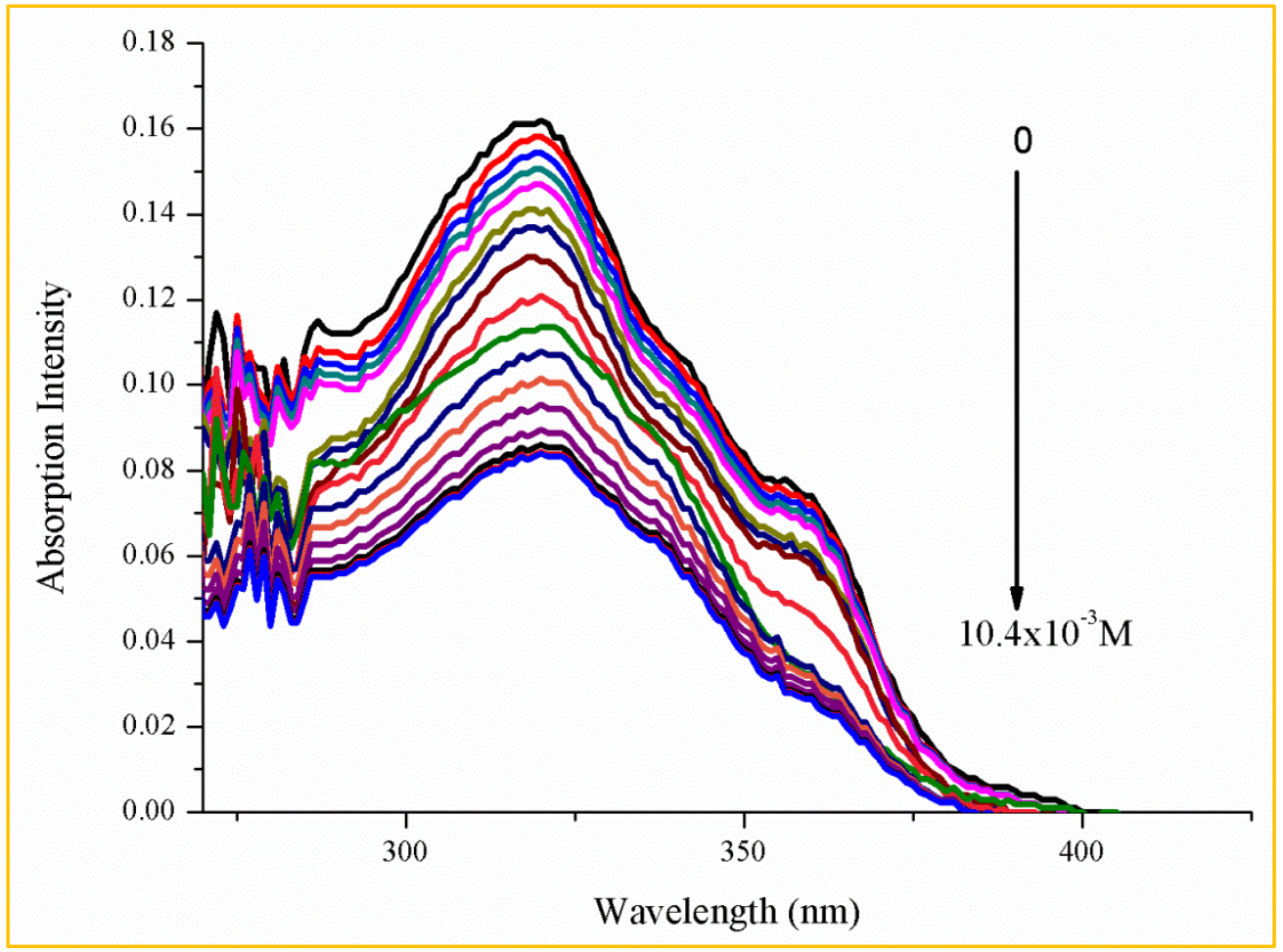

Figure S122. UV titration spectra of 11 with $\mathrm{CN}^{-}\left(0-10.4 \times 10^{-3} \mathrm{M}\right)$ in toluene. (Host $\left.\mathrm{C}=1.0 \times 10^{-5} \mathrm{M}\right)$ 


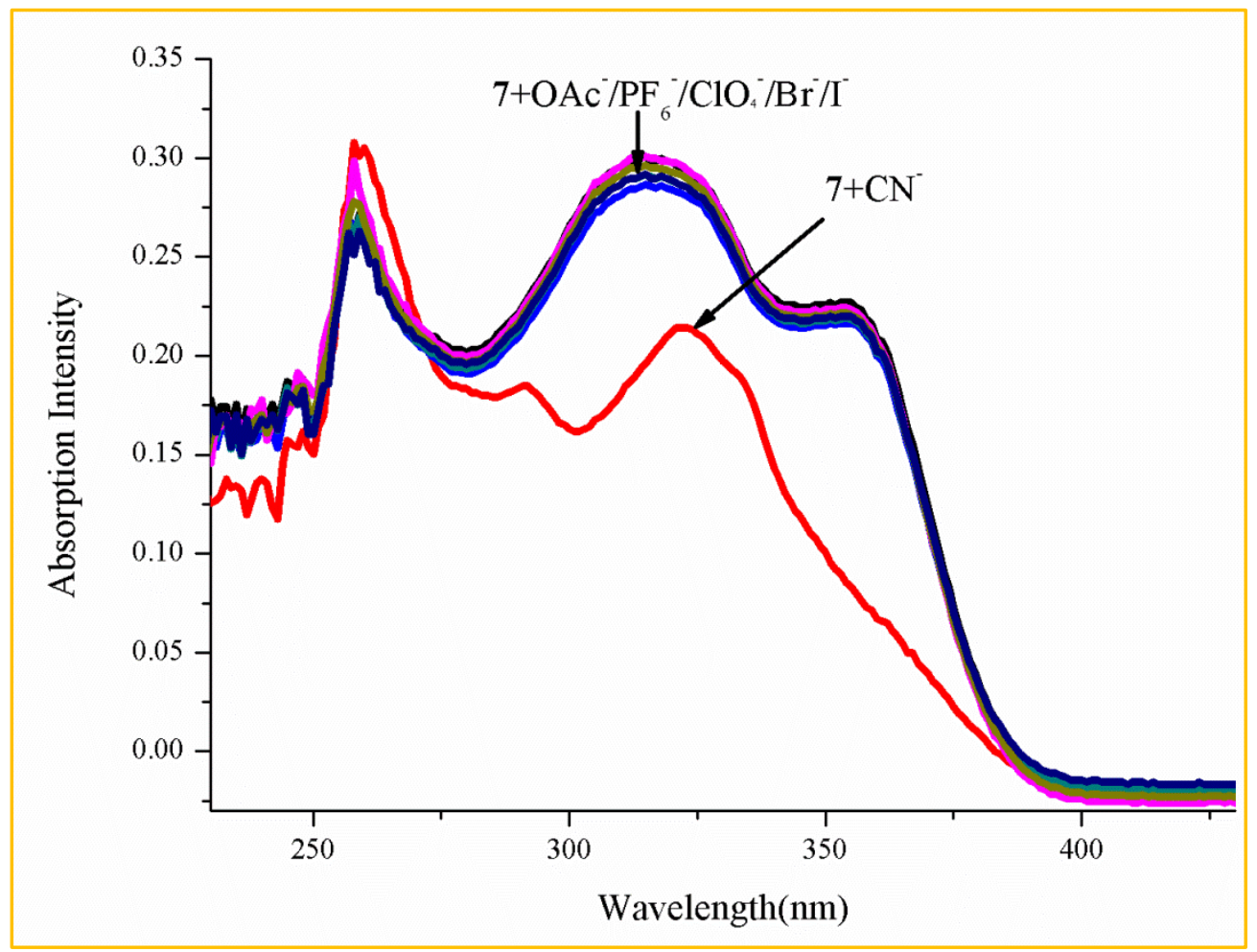

Figure S123. UV spectra of 7 containing various anion 100 eq. (as tetrabutylmmonium salts) in DMSO solution. (Host 7, $\mathrm{C}=1.0 \times 10^{-5} \mathrm{M}$ ).

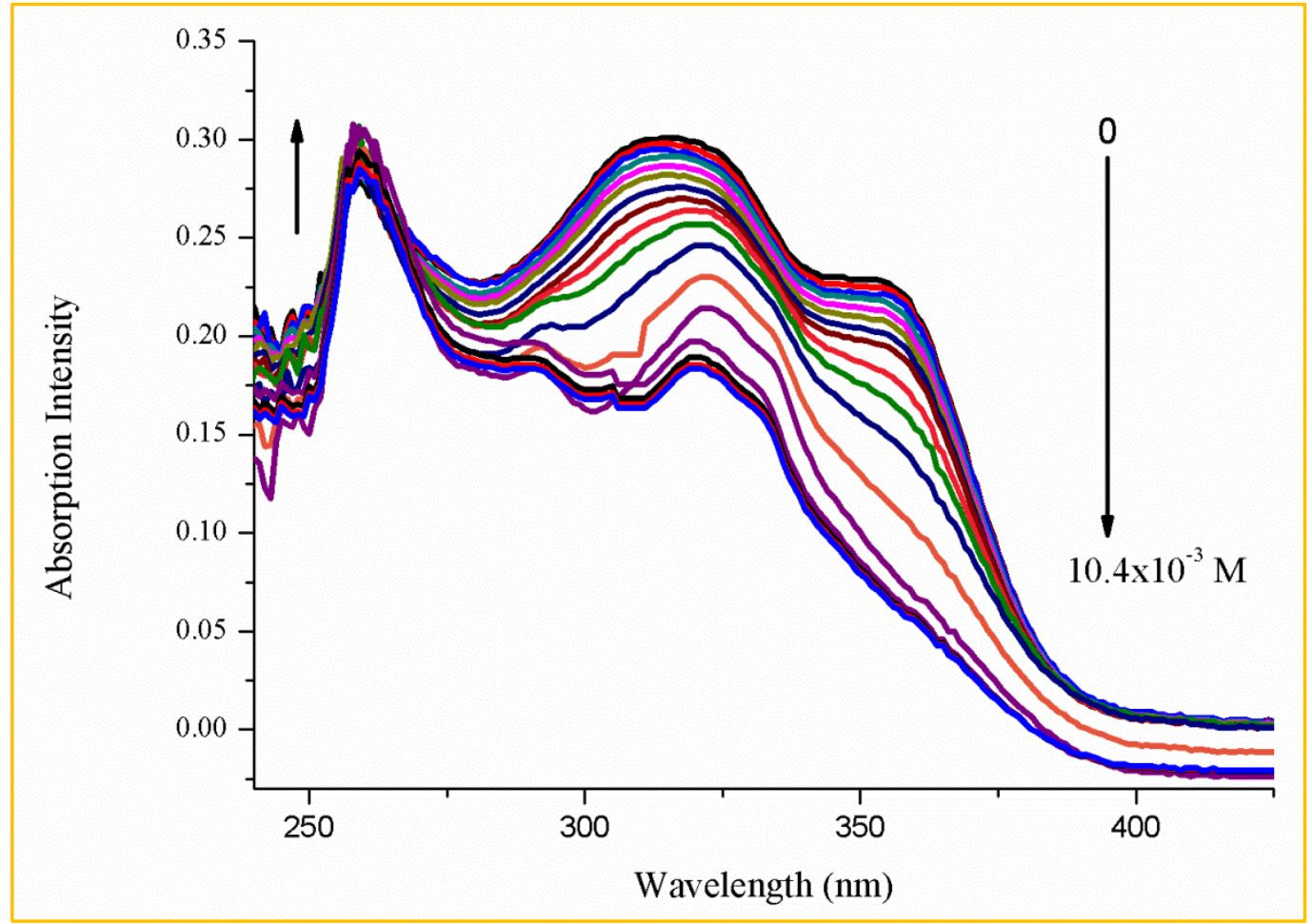

Figure S124. UV spectra of 7 upon gradual increase of $\mathrm{CN}^{-}\left(0-10.4 \times 10^{-3} \mathrm{M}\right)$ in DMSO. $\left(\right.$ Host $\left.\mathrm{C}=1.0 \times 10^{-5} \mathrm{M}\right)$ 


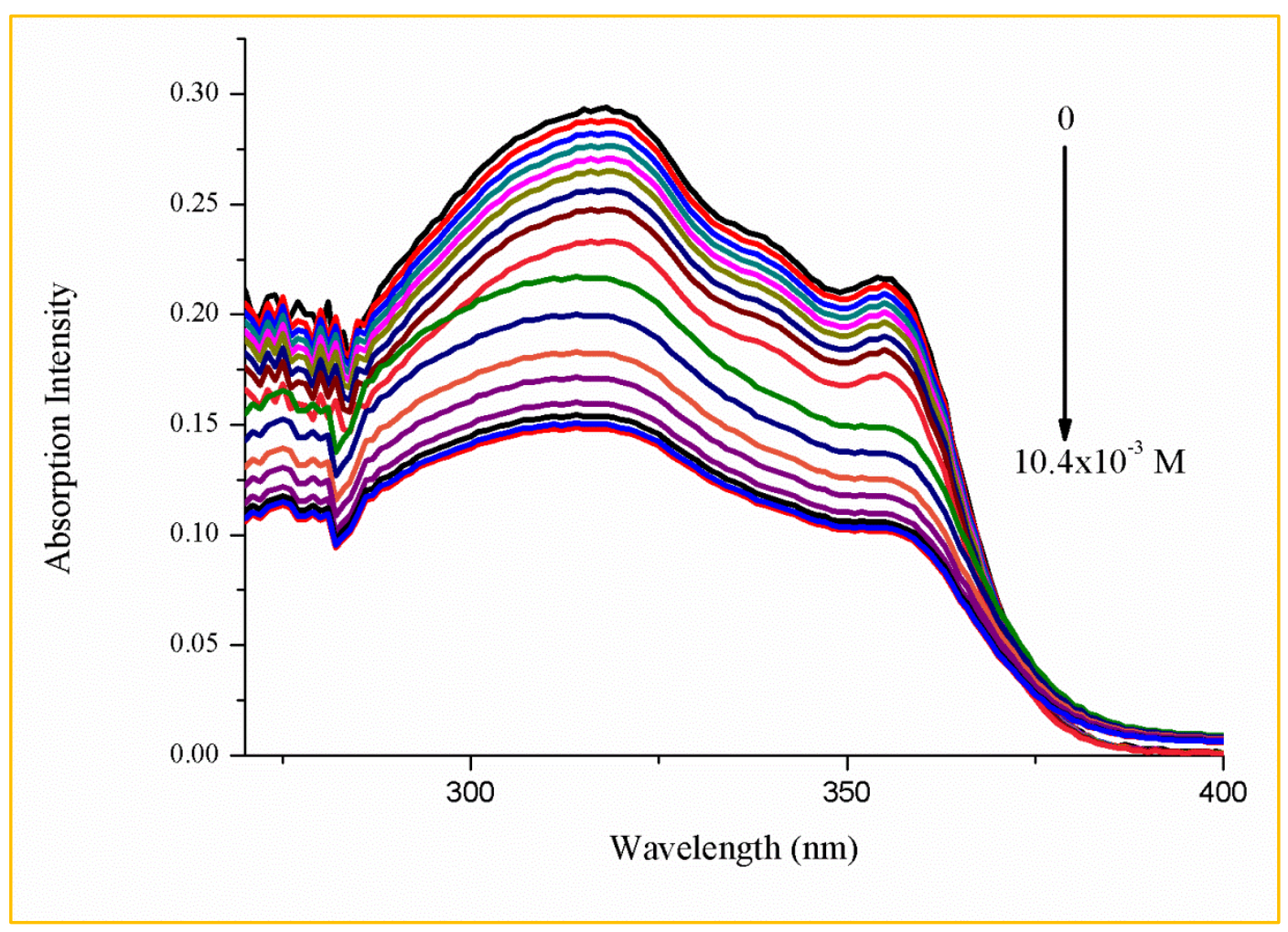

Figure S125. UV titration spectra of 7 with $\mathrm{CN}^{-}\left(0-10.4 \times 10^{-3} \mathrm{M}\right)$ in toluene. $\left(\right.$ Host $\left.\mathrm{C}=1.0 \times 10^{-5} \mathrm{M}\right)$ 


\section{NMR Sensing:}

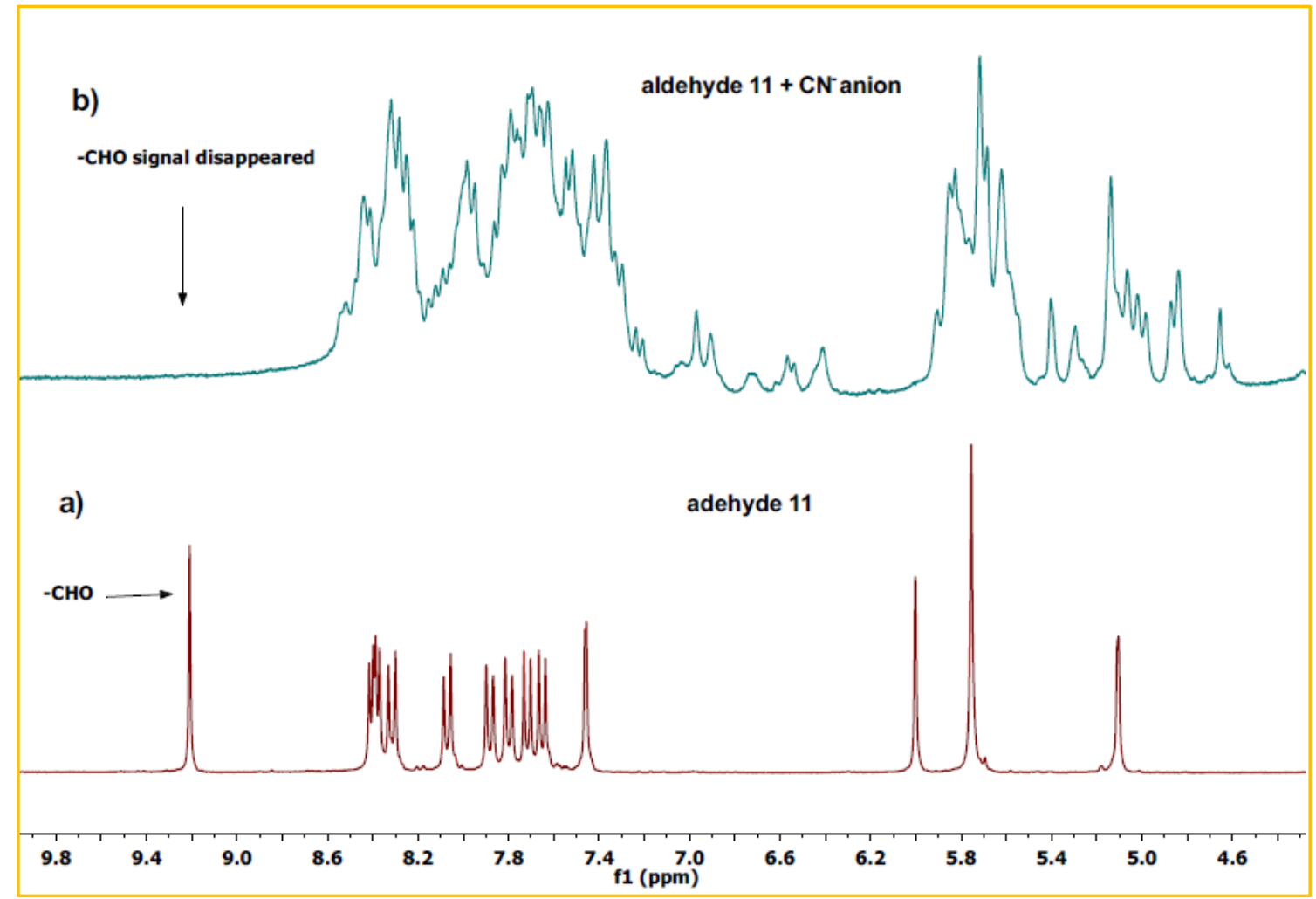

Figure S126. ${ }^{1} \mathrm{H}$ NMR of spectra of (a) $(M)$-monoaldehyde, 11; (b) (M)-monoaldehyde, $11+1$ eq. of cyanide anion in DMSO- $\mathrm{d}_{6}$ 